\title{
A Reconfigurable Architecture for Real-Time Image Compression On-Board Satellites
}

\author{
vorgelegt von \\ Dipl.-Inf. \\ Kristian Manthey \\ geb. in Wolfen
}

von der Fakultät IV - Elektrotechnik und Informatik

der Technischen Universität Berlin

zur Erlangung des akademischen Grades

Doktor der Ingenieurwissenschaften

- Dr.-Ing. -

genehmigte Dissertation

Promotionsausschuss:

Vorsitzender: Prof. Dr.-Ing. Thomas Sikora

Gutachter: Prof. Dr. Ben Juurlink

Gutachter: Prof. Dr.-Ing. Harald Michalik

Gutachter: Prof. Dr.-Ing. Reinhardt Karnapke

Tag der wissenschaftlichen Aussprache: 06. Oktober 2017

Berlin 2017 



\section{Abstract}

Data products of optical remote sensing systems are increasingly used in many areas of our everyday life. The spatial as well as the spectral resolution of satellite image data increases steadily with new missions resulting in a higher precision of known procedures and new application scenarios. While the memory capacity requirements can still be fulfilled, the transmission capacity becomes increasingly problematic. Real-time transmission of high-resolution image data is currently not possible.

This thesis presents a new image data compression architecture that can be used for current and future projects at the German Aerospace Center (DLR). The architecture has region-of-interest support and offers flexible access to the compressed data based on the CCSDS 122.0-B-1 image data compression standard. Region of interest (ROI) coding can be useful in scenarios where on-board classification, registration, or object or change detection algorithms are used. It is also useful to decrease the amount of data that must be transferred to the ground station. Modifications to the standard have been made to permit a change of compression parameters and the re-organization of the bit-stream after compression. An additional index of the compressed data is created, which makes it possible to locate individual parts of the bit-stream. On request, compressed and stored images can be re-assembled and transmitted according to the application's needs and as requested by the ground station.

The requirements, the design of the architecture, and its implementation based on reconfigurable hardware are presented. The architecture was developed for a spacequalified Xilinx Virtex 5QV, where a single instance of the architecture is capable of compressing images at a rate of up to $200 \mathrm{Mpx} / \mathrm{s}$ (or $400 \mathrm{Mbyte} / \mathrm{s}$ for 16 bit images). It operates at a clock frequency of $100 \mathrm{MHz}$ and processes two image pixels per clock cycle. A Xilinx Virtex-5QV enables thereby compressing images with a width of up to 4096 pixels without the use of external memory. Without external memory and additional interfaces, the power consumption of the architecture is about $5 \mathrm{~W}$. The proposed architecture is one of the fastest implementations yet reported and sufficient for recent high-resolution systems. Investigations in the resource and power consumption, as well as the availability of external storage have shown that it should be possible to integrate the design directly on a focal plane. 



\section{Zusammenfassung}

Datenprodukte von optischen Fernerkundungssystemen finden zunehmend Anwendung in unserem täglichen Leben. Die räumliche als auch die spektrale Auflösung von Satellitenbilddaten steigt mit neuen Missionen stetig, was zu einer höheren Präzision der bekannten Verfahren sowie neuen Anwendungsszenarien führt. Während die Anforderungen an die Speicherkapazität noch erfüllt werden können, wird die Übertragungskapazität zunehmend problematisch. Eine Echtzeit-Übertragung hochaufgelöster Bilddaten ist derzeit nicht möglich.

Diese Arbeit stellt eine neue Architektur zur Bilddatenkompression vor, welche für aktuelle und zukünftige Projekte am Deutschen Zentrum für Luft- und Raumfahrt (DLR) verwendet werden kann. Die Architektur besitzt eine Unterstützung für so genannte Regions of Interest und ermöglicht einen flexiblen Zugriff auf die mit dem Standard $C C S D S$ 122.0-B-1 komprimierten Bilddaten. Eine Region of Interest-Kodierung kann bei Anwendungen nützlich sein, bei denen Anbord-Klassifizierung oder -Registrierung, sowie Objekt- oder Änderungsdetektionsalgorithmen eingesetzt werden. Sie ist auch nützlich, um die Datenmenge, welche an die Bodenstation übertragen werden muss, zu reduzieren. Erweiterungen des Standards wurden vorgenommen, um eine Veränderung der Kompressionsparameter und die Neuorganisation des Bitstroms nach der Kompression zu ermöglichen. Es wird zusätzlich ein Index der komprimierten Daten erstellt, welcher es ermöglicht, einzelne Teile des Bitstroms zu lokalisieren. Auf Wunsch können die komprimierten und gespeicherten Bilder nach den Anforderungen der Anwendung und wie von der Bodenstation angefordert, zusammengesetzt und übertragen werden.

Die Anforderungen, das Design einer Architektur sowie deren Implementierung auf Basis von rekonfigurierbarer Hardware werden vorgestellt. Die Architektur wurde für einen raumfahrt-qualifizierten Xilinx Virtex 5QV entwickelt, wobei eine einzelne Instanz der Architektur in der Lage ist, Bilder mit einer Rate von bis zu 200 Mpx/s zu komprimieren. Sie arbeitet mit einer Taktfrequenz von $100 \mathrm{MHz}$ und prozessiert dabei zwei Bildpunkte pro Taktzyklus. Ein Xilinx Virtex-5QV ermöglicht dabei die Komprimierung von Bildern mit einer Breite von bis zu 4096 Bildpunkten ohne die Verwendung von externem Speicher. Ohne externen Speicher und zusätzliche Schnittstellen liegt die Leistungsaufnahme der Architektur bei etwa $4 \mathrm{~W}$. Bei der vorgestellten Architektur handelt es sich um eine der schnellsten Implementierungen, die bisher existieren, welche zudem für aktuelle hochauflösende Systeme geeignet ist. Untersuchungen des Ressourcen- und Energieverbrauchs, sowie bei der Verfügbarkeit externer Speicher haben gezeigt, dass es möglich sein sollte, das Design sensornah direkt auf einer Fokalebene zu integrieren. 



\section{Contents}

$\begin{array}{ll}\text { Acronyms } & 7\end{array}$

$\begin{array}{lll}1 & \text { Introduction } & \mathbf{1 1}\end{array}$

1.1 Historical remarks . . . . . . . . . . . . . . . . . . . . . . . . 11

1.2 Motivation . . . . . . . . . . . . . . . . 13

1.3 Contribution . . . . . . . . . . . . . . . . . 18

1.4 Organization . . . . . . . . . . . . . . . . 20

2 Fundamentals and Related Work $\quad 21$

2.1 Optical Remote Sensing . . . . . . . . . . . . . . . . . . . . 21

2.1 .1 Optical Sensor Systems . . . . . . . . . . . . . . . 21

2.1.1.1 Components . . . . . . . . . . . . . . . 22

2.1.1.2 Parameters .................. 26

2.1 .2 Satellite Imaging . . . . . . . . . . . . . . . . . . . . . . . . . . . . . . . . . . . . . . 38

2.2 Image Data Compression . . . . . . . . . . . . . . . . . . . . . . . 31

2.2.1 Basic Concepts . . . . . . . . . . . . . . . . . 31

2.2.1.1 Information Theory . . . . . . . . . . . . 31

2.2.1.2 Data Compression Techniques . . . . . . . . . . . 33

2.2.1.3 Coding ..................... 35

2.2.2 Wavelet Transform . . . . . . . . . . . . . . . . . 36

2.2.2.1 Discrete Wavelet Transform . . . . . . . . . . . . . . 37

2.2.2.2 Wavelet Filter . . . . . . . . . . . . . . . . . 38

2.2.2.3 The Lifting Scheme . . . . . . . . . . . . . . . . . . 39

2.2.2.4 Integer Wavelet Transform . . . . . . . . . . . . . . . . . 40

2.2.2.5 Wavelet Transform of Images . . . . . . . . . . . . . . . 41

2.2.3 Wavelet-Based Image Compression Algorithms . . . . . . . . . . . 43

2.2.4 Image Quality Assessment . . . . . . . . . . . . . . . . . . . 45

2.2.4.1 Statistical Methods . . . . . . . . . . . . . 45

2.2.4.2 Systems Theory Approach . . . . . . . . . . . . 47

2.3 Hardware Architectures . . . . . . . . . . . . . . . . . . . . 47

2.3.1 FPGA Hardware Architectures for Discrete Wavelet Transform . . 47

2.3.1.1 FPGA-Based One-Dimensional DWT . . . . . . . . . . 47

2.3.1.2 FPGA-Based Two-Dimensional DWT . . . . . . . 49

2.3.1.3 FPGA-Based Three-Dimensional DWT . . . . . . . . 50

2.3.2 Hardware Architectures for On-Board Image Data Compression . 50

2.3.2.1 ASIC-Based Compression Architectures . . . . . . . . . 51

2.3.2.2 COTS Hardware for On-Board Compression . . . . . . . 52 
2.3.2.3 FPGA-Based Compression Architectures . . . . . . . . . 52

2.3.3 Hardware Operating System . . . . . . . . . . . . . . . . . . 55

2.3.3.1 Channel Communication Protocol . . . . . . . . . . 55

2.3.3.2 SChannel Communication Protocol . . . . . . . . . 55

2.3.3.3 Pipe Communication Protocol . . . . . . . . . . 56

2.4 The CCSDS 122.0-B-1 Image Compression Standard . . . . . . . . . . . 56

2.4.1 Structure of the Compressor . . . . . . . . . . . . . . 57

2.4.2 Wavelet Transform Stage . . . . . . . . . . . . . . . . . 58

2.4.3 Block Decomposition Stage . . . . . . . . . . . . . . 59

2.4.4 Coding Stages . . . . . . . . . . . . . . . . . . . 59

2.4.4.1 Preparation Steps . . . . . . . . . . . . . 59

2.4.4.2 Segment Header . . . . . . . . . . . . . . . . . 61

2.4.4.3 Initial Coding . . . . . . . . . . . . . . . . . . 62

2.4.4.4 Bitplane Coding ................. 63

2.5 Summary . . . . . . . . . . . . . . . . . 65

3 Extensions of the CCSDS 122.0-B-1 Image Compression Standard 67

3.1 Region-Of-Interest Coding . . . . . . . . . . . . . . . . 67

3.1 .1 Unit of Information . . . . . . . . . . . . . . . . . 68

3.1 .2 Partitioning Schemes . . . . . . . . . . . . . . . . 70

3.2 Scalability . . . . . . . . . . . . . . . . . . 71

3.2.1 Analysis of the Compression Parameters . . . . . . . . . . . . . 72

3.2.1.1 Length and Size of the Resulting Bit-Stream Parts . . . 73

3.2 .2 Bit-Stream Indexing . . . . . . . . . . . . . . . . . . . . 75

3.2 .3 Bit-Stream Re-Assembling . . . . . . . . . . . . . . . . . . . . 77

3.3 Multi-spectral Image Compression . . . . . . . . . . . . . . . . 78

3.3.1 Spectral Decorrelation . . . . . . . . . . . . . . . 78

3.3.2 Multi-Channel Entropy Coding . . . . . . . . . . . . . . . . . 81

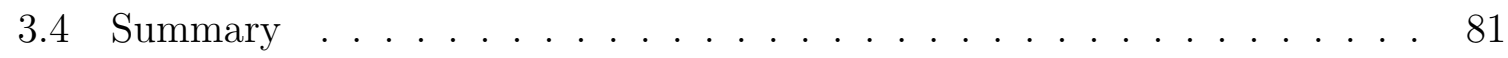

4 A New Image Data Compression Architecture 83

4.1 Quantitative Requirements . . . . . . . . . . . . . . . . . . . . . 83

4.2 Quantitative Requirements Analysis . . . . . . . . . . . . . . . . 85

4.3 Structure of the proposed architecture . . . . . . . . . . . . 86

4.3.1 Compression Controller . . . . . . . . . . . . . . . . 88

4.3.2 Wavelet Transform Module . . . . . . . . . . . . . . . 89

4.3.2.1 Row and Column DWT Module . . . . . . . . . . . . . 90

4.3.2.2 Data Rates and Memory Demands . . . . . . . . . . . . 91

4.3.2.3 Arithmetic Pipelines . . . . . . . . . . . . . . . . 93

4.3.2.4 Parallel Border-Less DWT . . . . . . . . . . . . . . . 94

4.3.3 Coefficient Grouping Module . . . . . . . . . . . . . . . . . 95

4.3.3.1 DWT Coefficient Input Module . . . . . . . . . . . . 96

4.3.3.2 Coefficient Memory Controller Module . . . . . . . . . . 96

4.3.3.3 Segment Formation Module . . . . . . . . . . . . 97 
4.3.3.4 Segment Buffer Module . . . . . . . . . . . . . . . 98

4.3.4 BitPlaneEncoder Module . . . . . . . . . . . . . . . . . . 98

4.3.4.1 BPE Control Module . . . . . . . . . . . . . . 98

4.3.4.2 Segment Header Module . . . . . . . . . . . . . . 100

4.3.4.3 QuantizedDC and BitDepthAC Module . . . . . . . . 101

4.3.4.4 Additional DC Bit-Planes Module . . . . . . . . . . . . 101

4.3.4.5 Stage Coding Modules . . . . . . . . . . . . . . . . 102

4.3.4.6 Output Muxer Module . . . . . . . . . . . . . . . 103

4.3.5 Memory Controller Module . . . . . . . . . . . . . . . . . . . . 104

4.3.5.1 Available Memory Resources . . . . . . . . . . . . . . . . 105

4.3.5.2 Concept for Using External Memory . . . . . . . . . . . 106

4.3.5.3 Integration Into the Design . . . . . . . . . . . . . . 107

4.4 Summary . . . . . . . . . . . . . . . . . . . . . . . . 109

5 Experimental Evaluation 111

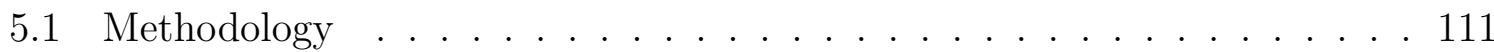

5.2 Overhead of the Index Table for ROI Coding . . . . . . . . . . . . . . . . 112

5.3 Data Compression Throughput . . . . . . . . . . . . . . . . 113

5.4 Resource Consumption and Maximum Clock Frequency . . . . . . . . . 116

5.4.1 Internal Precision of the Arithmetic . . . . . . . . . . . . . . . 117

5.4.2 Virtex-5 Synthesis Results . . . . . . . . . . . . . . . . . . 117

5.4.3 Kintex-7 Synthesis Results . . . . . . . . . . . . . . . . . . 119

5.5 Power Consumption . . . . . . . . . . . . . . . . . . . 120

5.6 Comparison with Other Implementations . . . . . . . . . . . . . . . . 122

5.7 Impact of Lossy Image Compression . . . . . . . . . . . . . . . . . . 124

5.7.1 Compression and Feature Matching . . . . . . . . . . . . . . . . . 124

5.7 .2 Compression and Stereo-Matching . . . . . . . . . . . . . . . 125

5.7 .3 Impact of Compression on Image MTF . . . . . . . . . . . . . . . 126

5.8 Summary . . . . . . . . . . . . . . . . . . . . 127

6 Conclusions and Future Work 129

6.1 Advanced Hardware Demonstrator . . . . . . . . . . . . . . . . . . 130

6.2 Integrated Focal Plane . . . . . . . . . . . . . . . . . . . . 130

6.3 Further Extensions for the Compression Algorithm . . . . . . . . . . . . 130

6.4 Improvements for the Compression Architecture . . . . . . . . . . . . . . 131

6.5 Towards Further On-Board Processing . . . . . . . . . . . . . . . . 131

$\begin{array}{ll}\text { References } & 133\end{array}$

$\begin{array}{ll}\text { A Additional Information } & \mathbf{1 4 5}\end{array}$

A.1 Hardware Design . . . . . . . . . . . . . . . . . . . 145

A.2 Pipe Communication Protocol . . . . . . . . . . . . . 145

A.3 Row Integer DWT . . . . . . . . . . . . . . . . . . . . . . 150

A.4 Row Floating Point DWT . . . . . . . . . . . . . . . 153 
A.5 Row Floating Point DWT (Lifting Layer) . . . . . . . . . . . . . . . . 157

B Test Images $\quad 161$

B.1 Synthetic Images . . . . . . . . . . . . . . . . . . . . . . . . 161

B.2 Leuven Images . . . . . . . . . . . . . . . . . . . . . . 162

B.3 Middlebury Images . . . . . . . . . . . . . . . . . . . . . . 162

B.4 CCSDS Reference Image Set . . . . . . . . . . . . . . . . 164

B.5 AVIRIS Hyper-Spectral Images . . . . . . . . . . . . . . . 171 


\section{List of Figures}

1.1 The first photo from space. . . . . . . . . . . . . . . . . 11

1.2 The first satellite photo taken by Explorer VI. . . . . . . . . . . . . . . . 12

1.3 A KOMPSat-3 half module and the focal plane assembly. . . . . . . . . . 13

1.4 Data processing chain of a conventional satellite imaging system. . . . . . 14

1.5 The GSD of some outstanding civil remote sensing satellites. . . . . . . . 15

1.6 The storage capacity of some outstanding civil remote sensing satellites. . 16

1.7 The downlink capability of some outstanding civil remote sensing satellites. 17

1.8 Concept of a new satellite image compression system. . . . . . . . . . . . 18

2.1 Optical remote sensing using a passive sensor system. . . . . . . . . . . . 22

2.2 Schematic of an optical sensor system on-board a space craft. . . . . . . . 22

2.3 Fundamentals of imaging by a thin lens. . . . . . . . . . . . . . . . . . 23

2.4 Imaging situation for an optical satellite. . . . . . . . . . . . . . . . . 24

2.5 Rate-distortion curve of raw and radiometric corrected data. . . . . . . . 25

2.6 Convolution of an object and a PSF. . . . . . . . . . . . . . . . 27

2.7 Rayleigh criterion for two point sources. . . . . . . . . . . . . . . 28

2.8 Principle terms of satellite imagery. . . . . . . . . . . . . . . . . . 29

2.9 Example of image collection scenarios with a single-sensor concept. . . . 30

2.10 Orbital and ground track speed of a satellite as a function of the altitude. 31

2.11 The symmetrical structure of a transform encoder and decoder. . . . . . 32

2.12 Sub-sampling of a signal. . . . . . . . . . . . . . . . . 33

2.13 Two-channel filter bank for signal analysis and synthesis. . . . . . . . . 35

2.14 Some basis functions of the Haar wavelet. . . . . . . . . . . . . . . . . . 37

2.15 Filter bank for signal analysis and synthesis (3 stages). . . . . . . . . . 38

2.16 Structure of the analysis filter of the $\operatorname{CDF}(9 / 7)$ wavelet. . . . . . . . . . . 41

2.17 Row and column transform of a two-dimensional DWT. . . . . . . . . . . 42

2.18 Three-stage two-dimensional discrete wavelet transform (DWT). . . . . . 42

2.19 Image marstest compressed at different compression ratios. . . . . . . . . 43

2.20 Parallel and quasi serial FIR filter. . . . . . . . . . . . . . . . . . . . . . . . . . 48

2.21 Structure of the Channel communication. . . . . . . . . . . . . . . 55

2.22 Structure of the SChannel communication. . . . . . . . . . . . . . 56

2.23 Structure of the Pipe communication. . . . . . . . . . . . . . 56

2.24 Overview on the structure of a CCSDS 122.0-B-1 Encoder. . . . . . . . 58

2.25 A block consisting of a DC coefficient and $63 \mathrm{AC}$ coefficients. . . . . . . . 60

2.26 Program and data flow of the bit-plane encoder (BPE) . . . . . . . . . 61

2.27 Overview of the stage coding withing a segment. . . . . . . . . . 63 
3.1 An example using ROI coding. . . . . . . . . . . . . . . . . . 68

3.2 Discontinuities at the image borders when tiling in spatial domain. . . . 70

3.3 Rate-distortion curve for different segment sizes. . . . . . . . . . . . . . . 71

3.4 Different segment sizes and an exemplary region-of-interest. . . . . . . . . 71

3.5 A scene before and after compression and as requested by the ground station. . . . . . . . . . . . . . . . . . 72

3.6 Average size of the encoded quantized DC coefficients. . . . . . . . . . . . 74

3.7 Average size of the encoded bit-depth of the AC coefficients. . . . . . . . 75

3.8 Distribution of individual bits within the compressed bit-stream. . . . . . 76

3.9 On-board re-assembling of the bit-stream. . . . . . . . . . . . 78

3.10 On-board assembling of an update for an already transferred image. . . . 78

3.11 Independent processing of several spectral channels. . . . . . . . . . . . 79

3.12 The spectral channels are decorrelated and then compressed independently. 79

3.13 Correlation of the 224 spectral channels of aviris scene 0 (cal). . . . . . . 80

3.14 The spectral channels are decorrelated and then compressed jointly. . . . 81

4.1 Overview on the proposed architecture for multi-spectral compression. . . 84

4.2 Structure of the proposed architecture. . . . . . . . . . . . . . 87

4.3 Structure of the top-level Wavelet Transform Module (WTM). . . . . . . 90

4.4 Structure of a single 2D-DWT module. . . . . . . . . . . . . . . . 90

4.5 Overview of the structure of the row DWT. . . . . . . . . . . . . 91

4.6 Overview of the structure of the column DWT. . . . . . . . . . . . . . 91

4.7 Structure of the Integer DWT pipeline. . . . . . . . . . . . . . . . . 93

4.8 Structure of the Floating Point (FP) DWT pipeline. . . . . . . . . . . . . 94

4.9 Overview on the approach for the parallel border-less DWT. . . . . . . . 95

4.10 Structure of the Coefficient Grouping Module. . . . . . . . . . . . . . . 96

4.11 Overview on the proposed BitPlaneEncoder Module. . . . . . . . . . . . 99

4.12 Overview on the BPE Control Module. . . . . . . . . . . . . . . . . 100

4.13 Overview on the DPCM Module. . . . . . . . . . . . . . . . . 101

4.14 Overview on the Stages 1, 2, and 3 Coding Module. . . . . . . . . . . 103

4.15 Overview on the Output Muxer Module. . . . . . . . . . . . . . . . . 104

4.16 Principles of the Output-Shifter Module. . . . . . . . . . . . . . . 105

4.17 Pipe Delay Module. . . . . . . . . . . . . . . . . . . . . . . . 107

4.18 Multi-Pipe Delay Module with SRAM memory controller. . . . . . . . . . 108

4.19 Concept of using external memory. . . . . . . . . . . . . . . 108

5.1 Qualitative comparison of the 24-bit and 32-bit floating-point DWT. . . . 118

5.2 Power consumption of the design on a Virtex-5 and Virtex-5QV FPGA. . 121

5.3 Impact of image compression on feature matching. . . . . . . . . . . . . 125

5.4 Impact of image compression on stereo-matching. . . . . . . . . . . . 127

5.5 Impact of image compression on the MTF . . . . . . . . . . . . 128

A.1 The Gajski-Kuhn Y-chart model. . . . . . . . . . . . . . . . . 146

B.1 Middlebury Stereo Vision test images. . . . . . . . . . . . . . . 163 
B.2 CCSDS reference image coastal. . . . . . . . . . . . 165

B.3 CCSDS reference image lunar. . . . . . . . . . . . . . . 166

B.4 CCSDS reference image marstest. . . . . . . . . . . . . . 166

B.5 CCSDS reference image india_2kb1. . . . . . . . . . . . . . 166

B.6 CCSDS reference image india_2kb4. . . . . . . . . . . . 166

B.7 CCSDS reference image ocean_2kb1. . . . . . . . . . . . . . 167

B.8 CCSDS reference image ocean_2kb4. . . . . . . . . . . . . . . 167

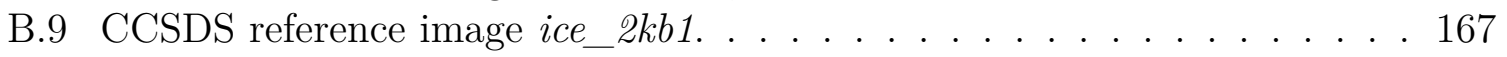

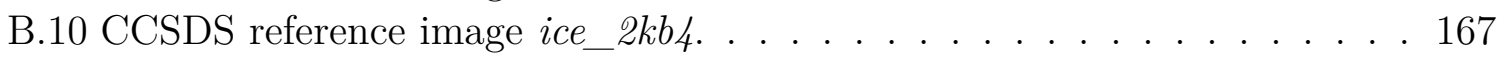

B.11 CCSDS reference image solar. . . . . . . . . . . . . . . . . . . . . . . . . . . . . . . . . . . . . .

B.12 CCSDS reference image sun_spot. . . . . . . . . . . . . . . . . 168

B.13 CCSDS reference image $w f p c \ldots \ldots \ldots . \ldots \ldots$

B.14 CCSDS reference image foc. . . . . . . . . . . . . . . . . . . . . . . . . . . . . . . . . . . . . .

B.15 CCSDS reference image pleiades_portdebouc_pan. . . . . . . . . 168

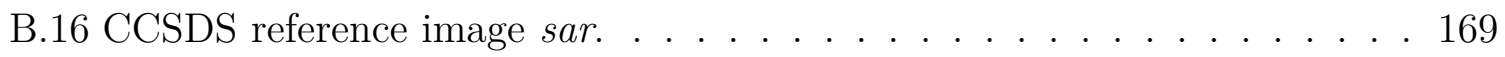

B.17 CCSDS reference image p160_b_f. . . . . . . . . . 169

B.18 CCSDS reference image europa3. . . . . . . . . . . . . . . 169

B.19 CCSDS reference image spot-la_b3. . . . . . . . . . . . . . . . 169

B.20 CCSDS reference image pleiades_portdebouc_b3. . . . . . . . . . 169

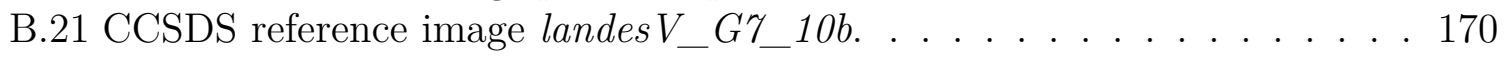

B.22 CCSDS reference image marseille_G6_10b. . . . . . . . . . . . . 170 



\section{List of Tables}

2.1 Filter coefficients of commonly used wavelet filters. . . . . . . . . . . . 39

2.2 Compression algorithms employed for space missions. . . . . . . . . . . . 45

2.3 application-specific integrated circuit (ASIC)-based compressors for space applications. . . . . . . . . . . . . . . . . . . 52

2.4 Compression-related algorithm implementations using GPGPU. . . . . . 53

2.5 field-programmable gate array (FPGA)-based compressors for space applications. . . . . . . . . . . . . . . . . 55

3.1 Comparison of using image-, segment-, gaggle- or block-based ROI coding. 69

3.2 Quality-related compression parameters of CCSDS 122.0-B-1. . . . . . . 73

4.1 Comparison of the Xilinx Virtex 5QV internal memory resources [128]. . 105

4.2 A comparison of some state-of-the-art memory technologies available for space applications. . . . . . . . . . . . . . . . . 106

5.1 Size of the index compared to the size of the input image. . . . . . . . . 113

5.2 Data compression throughput for lossless compression (Integer only). . . 114

5.3 Data compression throughput for near-lossless compression (FP and Integer

DWT architecture). . . . . . . . . . . . . . . . . . 116

5.4 FPGA resource consumption (Virtex-5, $I_{w}=1024 \mathrm{px}$, Integer DWT only). 119

5.5 FPGA resource consumption (Virtex-5, $I_{w}=2048 \mathrm{px}$, Integer DWT only). 119

5.6 FPGA resource consumption (Virtex-5, $I_{w}=4096 \mathrm{px}$, Integer DWT only). 120

5.7 FPGA resource consumption (Virtex-5, $I_{w}=4096 \mathrm{px}$, Integer and FP DWT) . . . . . . . . . . . . . . . . . 120

5.8 FPGA resource consumption (Kintex-7, $I_{w}=4096 \mathrm{px}$, Integer and FP DWT) . . . . . . . . . . . . . . . . . 121

5.9 Comparison of execution times and data throughput for original images. 122

5.10 Comparison of execution times and data throughput for 16 times concatenated image-set. . . . . . . . . . . . . . . . . . . . 123

5.11 Comparison of image data compression implementations. . . . . . . . . . 123

B.1 CCSDS Reference image set. . . . . . . . . . . . . . . 164

B.2 AVIRIS hyper-spectral images. . . . . . . . . . . . . . . . 171 



\section{Acronyms}

$\begin{array}{ll}\text { ADC } & \text { analog-to-digital converter } \\ \text { AHP } & \text { analysis high-pass filter } \\ \text { ALP } & \text { analysis low-pass filter } \\ \text { ASIC } & \text { application-specific integrated circuit } \\ \text { ASWDR } & \text { Adaptively Scanned Wavelet Difference Reduction } \\ & \\ \text { BPE } & \text { bit-plane encoder } \\ \text { BPEM } & \text { BitPlaneEncoder Module } \\ \text { BTC } & \text { block truncation coding } \\ \text { BWCP } & \text { Bandwidth Compression Plus } \\ & \\ \text { CABAC } & \text { context-adaptive binary arithmetic coding } \\ \text { CCD } & \text { charge-coupled device } \\ \text { CCM } & \text { Compression Controller Module } \\ \text { CCSDS } & \text { Consultative Committee for Space Data Systems } \\ \text { CDF } & \text { Cohen-Daubechies-Feauveau } \\ \text { CGM } & \text { Coefficient Grouping Module } \\ \text { CMOS } & \text { complementary metal-oxide semiconductor } \\ \text { CNES } & \text { Centre national d'études spatiales } \\ \text { CoReCi } & \text { Compression Recording and Ciphering } \\ \text { COTS } & \text { components-off-the-shelf } \\ \text { CPU } & \text { central processing unit } \\ \text { CR } & \text { compression ratio } \\ \text { CUDA } & \text { Compute Unified Device Architecture } \\ \text { CWICOM } & \text { CCSDS Wavelet Image COmpression Module } \\ & \\ \text { DCT } & \text { discrete cosine transform } \\ \text { DHT } & \text { discrete Hartley transform } \\ \text { DLR } & \text { German Aerospace Center, German: Deutsches Zen- } \\ & \text { trum für Luft- und Raumfahrt e. V. } \\ \text { DN } & \text { digital number } \\ \text { DPCM } & \text { differential pulse code modulation } \\ \text { DSP } & \text { digital signal processor } \\ \text { DWT } & \text { discrete wavelet transform } \\ & \\ \text { EBCOT } & \text { Embedded Block Coding with Optimized Truncation }\end{array}$


EDAC error detection and correction

ESF edge spread function

EZW Embedded Zerotree of Wavelet transforms

FIFO First In, First Out

FIR finite impulse response

FMC forward motion compensation

FP Floating Point

FPA focal plane assembly

FPGA field-programmable gate array

FSM finite state machine

GPGPU general-purpose computation on graphics processing unit

GPU graphics processing unit

GSD ground sample distance

HEVC High Efficiency Video Coding

HRSST Helmholtz Research School on Security Technologies

HS hyper-spectral

HUB Humboldt-Universität zu Berlin

ICM Image Compression Module

IDC image data compression

IFOV instantaneous field of view

IQA image quality assessment

JPIP JPEG2000 Interactive Protocol

KARI Korea Aerospace Research Institute

KLT Karhunen-Loève transform

LSF line spread function

MAE mean absolute error

MCM multi-chip module

MDM Multi-Pipe Delay Module

MEX MATLAB Executable

MMU memory management unit

MOS mean opinion score

MRCPB Multi-Résolution par Codage de Plans Binaires

MS multi-spectral

MSE mean square error

MSSIM mean structural similarity 
MTF modulation transfer function

PCA principal component analysis

PCB printed circuit board

PDF probability density function

PDHU Payload Data Handling Unit

PSF point spread function

PSNR peak signal-to-noise ratio

QE quantum efficiency

RHBD Radiation-Hardening-by-Design

ROI region of interest

SDRAM synchronous dynamic random-access memory

SEU single event upset

SHP synthesis high-pass filter

SIFT scale-invariant feature transform

SLP synthesis low-pass filter

SNR signal-to-noise ratio

SPECK set partitioned embedded block coder

SPIHT Set Partitioning In Hierarchical Trees

SRAM static random-access memory

SSIM structural similarity

SSO sun-synchronous orbit

TC Telecommand

TID total ionizing dose

TM Telemetry

TMR triple modular redundancy

TUB Technische Universität Berlin

VHDL VHSIC Hardware Description Language

VLC variable-length code

WDR Wavelet Difference Reduction

WICOM Wavelet Image COmpression Module

WTM Wavelet Transform Module

XPE Xilinx Power Estimator 



\section{Introduction}

\subsection{Historical remarks}

When the Frenchman Joseph Nicéphore Nièpce probably made the first durable photo in 1826, nobody could foresee the development of photography in the following two centuries. Nièpce used a camera obscura ${ }^{1}$, a polished pewter plate, and an exposure time of approximately eight hours to capture a photo of the view from his office. 120 years later, a similar groundbreaking event happened:

On October 24, 1946, American scientists made the first pictures of Earth from space. A camera was installed on a V-2 rocket and took an image every $1.5 \mathrm{~s}$ in an altitude of around $105 \mathrm{~km}$. A few minutes later, the rocket began to fall back to Earth and slammed to the ground at a speed of $550 \mathrm{~km} / \mathrm{h}$. The camera was destroyed, but the film remained well protected in a steel cassette. Figure 1.1 shows one of the photos taken.

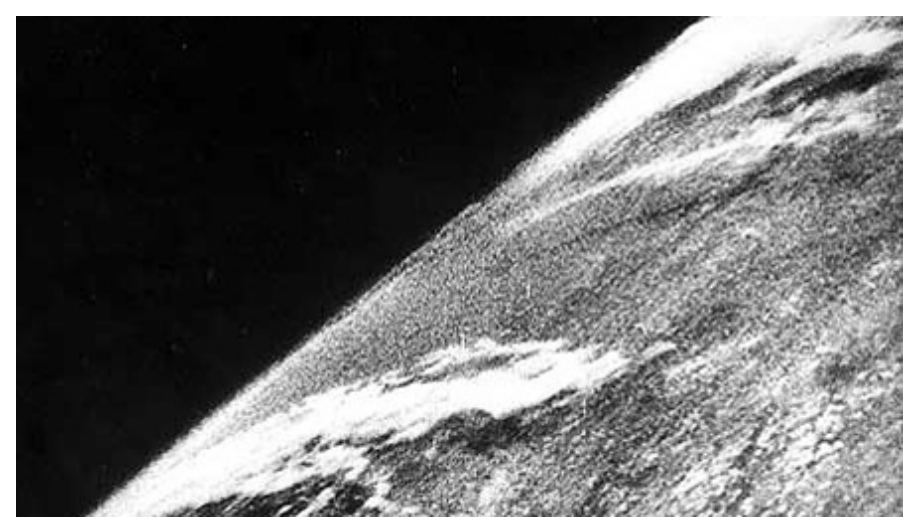

Figure 1.1: The first photo taken from space, Credit: U.S. Army (public domain).

Explorer VI, the first satellite with an on-board camera, was launched on August 14, 1959 - approximately two years after the first artificial satellite Sputnik 1. Compared with the former airborne images and today's satellite images, the images taken by Explorer VI were very poor (see Figure 1.2). At the same time, the USA started the CORONA project, a series of imaging reconnaissance satellites named Keyhole (KH). In 1992, the images taken by the CORONA satellites were declassified and became available for civilian usage.

Civilian satellite images are available since the launch of Landsat- $1^{2}$ in 1972 . Landsat-1 had a ground sample distance (GSD) of $80 \mathrm{~m}$ and was designed for Earth's resource exploration. The next big technology jump was made by SPOT-1 ${ }^{3}$ in 1986 . SPOT-1

\footnotetext{
${ }^{1}$ Archetype of a camera based on the principle of the pinhole camera

${ }^{2}$ Originally named Earth Resources Technology Satellite 1, ERTS-1

${ }^{3}$ French: Satellite Pour l'Observation de la Terre
} 
HRV $^{4}$ had a GSD of $10 \mathrm{~m}$. This resolution was first undercut by KOMPSat-1 with $6.6 \mathrm{~m}$ in 1999. In the same year, IKONOS was launched with a GSD of $0.82 \mathrm{~m}$.

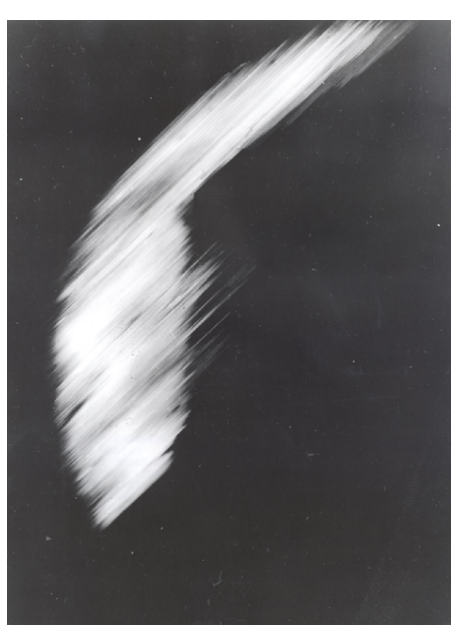

Figure 1.2: The first satellite photo taken by Explorer VI, Credit: NASA (public domain).

Nowadays, optical remote sensing systems are used in various safety and civil security applications for geology, archeology, reconnaissance, change detection, or for planetary research and astronomy. Disaster management after floodings or earthquakes, detection of environmental pollutions, or fires are examples of countless number of applications. The spatial, the spectral, the radiometric as well as the temporal resolution of satellite image data increases steadily with new missions resulting in higher precision and allowing new applications. On the technical side, there is a tremendous increase in data rate that has to be handled by satellite imaging systems. The space environment leads to further challenging requirements regarding reliability, power consumption, or constraints by the environment in which the system is supposed to be used. All these requirements lead to complex designs and high costs.

The expertise of the Institute of Optical Sensor Systems at the DLR ${ }^{5}$ is the development of sensors and data processing solutions for commercial, governmental, and scientific purposes. It has experience in developing remote sensing sensors for more than 20 years. Some institute's key products and applications are used today for the latest airborne and satellite technology. Several focal plane assemblies (FPAs) for projects such as Mars96 (WAOSS), ADS40, KOMPSat-3 or KOMPSat-3A have been developed. The last two are high-resolution satellite missions of the Korea Aerospace Research Institute (KARI) with a GSD of $70 \mathrm{~cm}$ or $55 \mathrm{~cm}$, respectively, In both missions, the high-resolution optical sensor, the FPA shown in Figure 1.3, was developed at the DLR.

In KOMPSat-3A, remote sensing images were acquired with a data rate of approximately $7 \mathrm{Gbit} / \mathrm{s}$ depending on the altitude of the satellite and the number of active spectral channels. On the FPA, image data are read from the detector and radiometric corrections were applied. Data compression, storage and formatting is done inside the Payload Data Handling Unit (PDHU). The system consists of other modules, such as the optical module, the camera controller, the power supplies and the radio module. Each of these modules has its own processors and redundancy structures. Because of this modularization and environmental constraints, these systems are very inflexible concerning adaptability to changing requirements. It has been shown in the project KOMPSat-3A, which is based on KOMPSat-3, that even small changes in the requirements or a shortage of qualified parts

\footnotetext{
${ }^{4}$ HRV: High Resolution Visible, imaging instrument on board SPOT-1

${ }^{5}$ German Aerospace Center, German: Deutsches Zentrum für Luft- und Raumfahrt e. V. (DLR)
} 
can lead to an almost complete new design. A good strategy to reduce mass, volume and costs is to integrate data (pre-)processing near the sensor. At the DLR, the vision is to develop a satellite camera that implements all tasks - image acquisition, pre-processing, compression, storage, data formatting and communication with the ground station - on a single multi-chip module (MCM). As a first step, hardware and software components of a smart image data compression system is to be developed.

\subsection{Motivation}

A recent high-resolution satellite imaging system captures images, applies pre-processing operations like radiometric corrections, stores lossy- or lossless-compressed images and transmits the data to a ground station. Usually, lossy compression is used for Earth observation, whereas scientific missions most often require lossless or near-lossless compression. Data post-processing or product generation is usually performed on-ground. Image compression is performed because of limited storage capacity or download capability. Figure 1.4 shows the structure of a recent satellite imaging system.

\section{Key parameters of recent systems}

The term high-resolution must be seen in the context of an application and is restricted by legislation, financial resources and by the current technical possibilities. Meteorological satellite cameras used for weather forecasting, for instance, have a GSD of approximately $1 \mathrm{~km}$, whereas today's multipurpose high-resolution satellites have a GSD of less than $1 \mathrm{~m}$. Figure 1.5 shows the GSD of some important remote sensing satellites. It is apparent that the resolution has increased over time. A GSD of significantly less than $50 \mathrm{~cm}$ can be expected for future systems. If the swath width ${ }^{6} S W$ remains constant, the sensor data rate increases quadratically with a decreasing GSD as shown in the following equation:

$$
\text { Data Rate }=\frac{1}{G S D^{2}} \cdot S W \cdot v_{\text {ground }}(h) \cdot \mathrm{DR}
$$
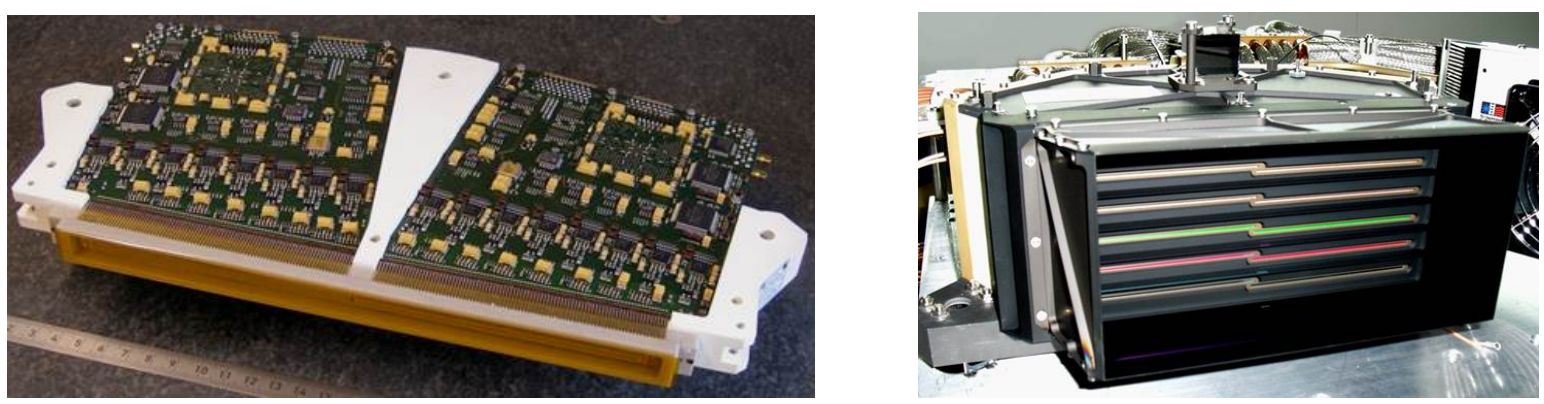

Figure 1.3: A KOMPSat-3 half module and the focal-plane assembly, Credit: (DLR/KARI).

\footnotetext{
${ }^{6}$ The swatch width is the stripe of the Earth's surface from which the image data is acquired (see
} Figure 2.8). 


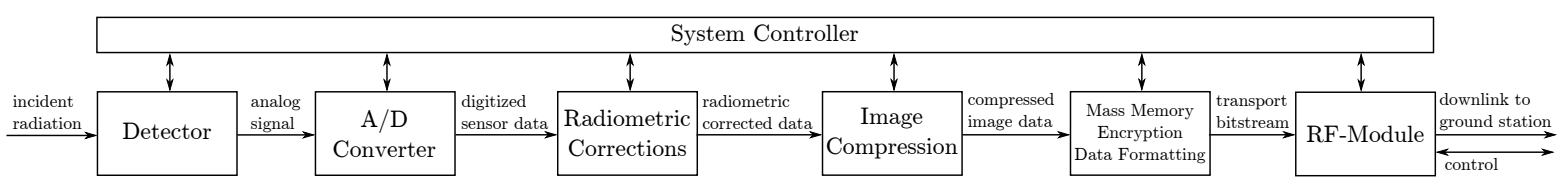

Figure 1.4: Data processing chain of a conventional satellite imaging system.

where $v_{\text {ground }}(h)$ is the ground speed of the satellite that depends on the altitude $h$, and $D R$ is the dynamic range of each sensor pixel.

In addition to the data rate, storage capacity and downlink capability are important parameters of a satellite imaging system. The storage capacity of a satellite is limited by mass and power restrictions and the availability of high-density memory parts. Today's remote sensing missions take advantage of recent developments in flash memory. Today, space-qualified NAND ${ }^{7}$ flash memory is available with a capacity of 64 Gbit per module. The development in memory devices is reflected in the storage capacity of remote sensing systems shown in Figure 1.6. It can be assumed, that the storage capacity on-board a future spacecraft will continue to increase. Since the acquired images have to be sent to the ground station, the downlink capability is a major limitation of the system. Figure 1.7 shows the downlink capability of some past and recent missions. It can be seen that the total downlink capability has increased significantly in the last years. Since radio transmission uses a shared medium, and since the number of satellites will increase in the future, it can be assumed, that progress in radio transmission technology will hardly compensate the increasing amount of data that has to be transferred to the ground station[114, 115]. Recent developments in optical transmission of data using lasers will probably relax this situation for a while.

\section{Problems of recent systems}

An exemplary high-resolution system has a GSD of $50 \mathrm{~cm}$ for the panchromatic channel and $1 \mathrm{~m}$ for 4 multi-spectral channels. Furthermore, it has a swath width of $12 \mathrm{~km}$ and a dynamic range of 12 bit per pixel. The ground speed of an orbital satellite is around $7 \mathrm{~km} / \mathrm{s}$. According to Equation (1.1), the total data rate of such a system is:

$$
\begin{aligned}
\text { total data rate } & =\frac{1}{(50 \mathrm{~cm})^{2}} \cdot 12 \mathrm{~km} \cdot 7 \mathrm{~km} / \mathrm{s} \cdot 12 \mathrm{bit}+4 \cdot \frac{1}{(1 \mathrm{~m})^{2}} \cdot 12 \mathrm{~km} \cdot 7 \mathrm{~km} / \mathrm{s} \cdot 12 \mathrm{bit} \\
& =\left(\frac{1}{(50 \mathrm{~cm})^{2}}+4 \cdot \frac{1}{(1 \mathrm{~m})^{2}}\right) \cdot 12 \mathrm{~km} \cdot 7 \mathrm{~km} / \mathrm{s} \cdot 12 \mathrm{bit} \\
& =672 \cdot 10^{6} \cdot 1 / \mathrm{s} \cdot 12 \mathrm{bit} \\
& =8.064 \mathrm{Gbit} / \mathrm{s}
\end{aligned}
$$

The memory capacity of this exemplary system could be approximately 2 Tbit [96, 97]. After lossless compression, the data rate is approximately $2 \mathrm{Gbit} / \mathrm{s}$ assuming a

\footnotetext{
${ }^{7}$ Typical architecture of flash memory devices.
} 


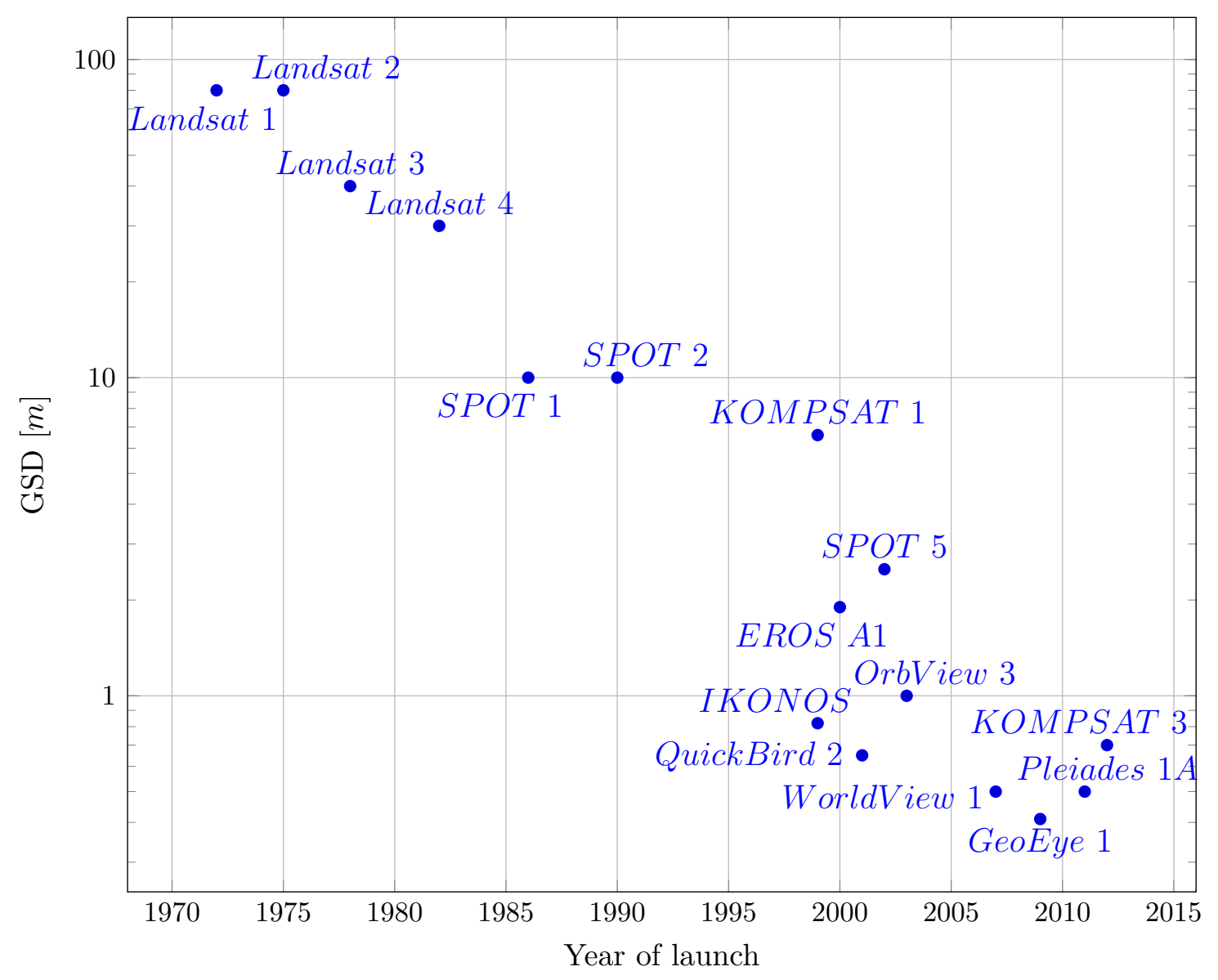

Figure 1.5: The GSD of some outstanding civil remote sensing satellites.

typical near-lossless compression ratio of $4: 1$, so that only approximately twenty minutes out of a hundred minutes orbit can be stored without any loss of information. The download capacity is also limited in throughput and time: All high-resolution satellite systems examined for this thesis use the X-band for image data transmission. For data transmission, an X-band antenna, with a downlink capability of approximately $800 \mathrm{Mbit} / \mathrm{s}$ can be used. The main problems of these systems are:

- A huge amount of data has to be compressed.

- Limited storage capacity: The duty cycle of an orbit is only approximately $20 \%$.

- Limited downlink capability: Transmission capability is limited by the radio channel. Transfer time per orbit is limited by the visibility of the ground station. Though there are approaches to use geostationary satellites to increase the download capability, these approaches further increase the costs.

In most applications, a region of interest (ROI) can be used to control the quality parameters of lossy image compression. Lossless or near-lossless compression can be used for important areas of an image whereas other areas are compressed with a lower quality. 


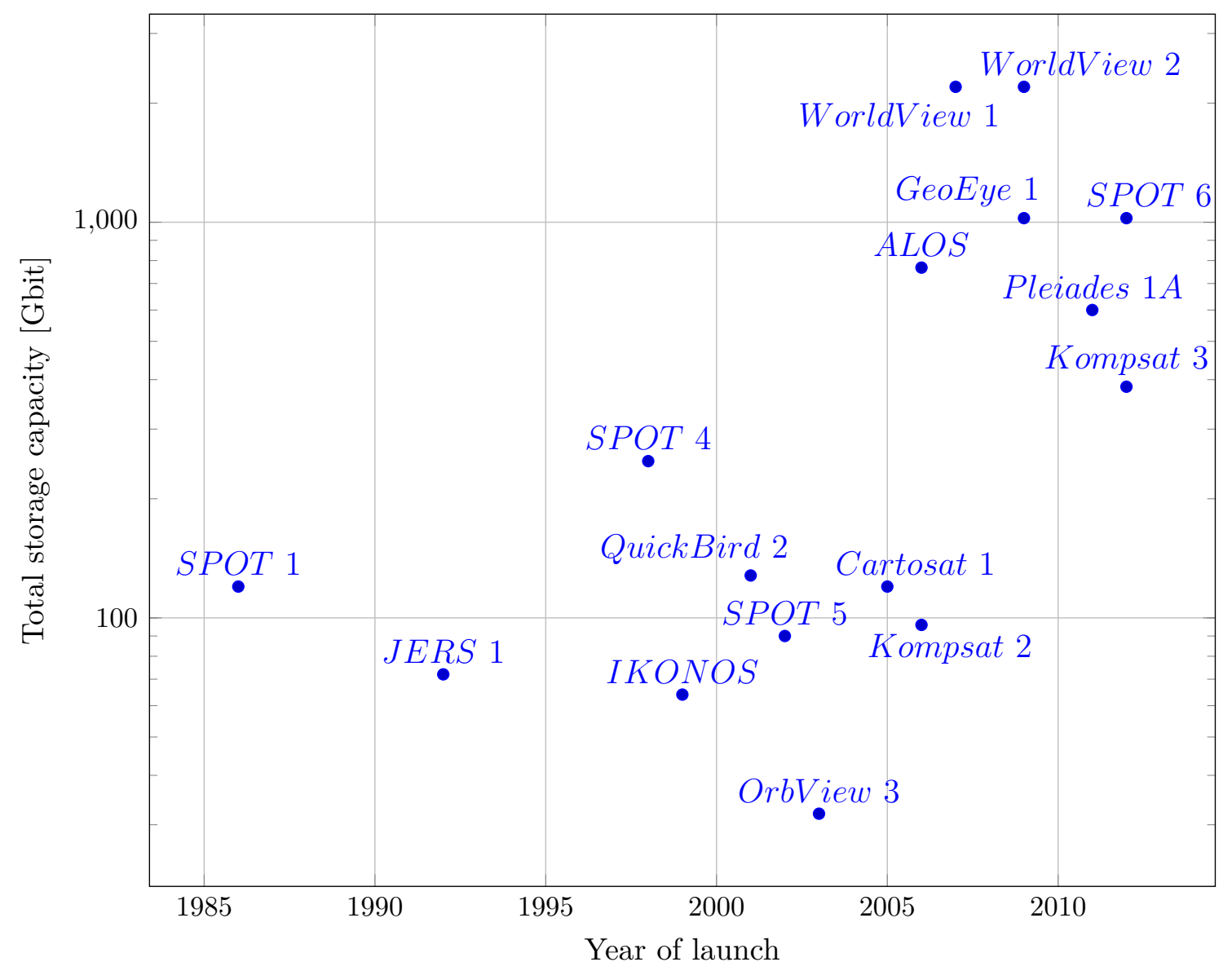

Figure 1.6: The storage capacity of some outstanding civil remote sensing satellites.

Near sensor data processing is a concept where a significant part of the data processing is performed near the detector in order to reduce the power consumption (e. g. caused by interconnects), the volume and mass as well as the costs of the device.

Airbus Defence and Space just finished the development of a new compression ASIC for space applications [25, 34]. Although the compression throughput is $60 \mathrm{Mpx} / \mathrm{s}$, ten of these ASICs would be just sufficient to compress the complete data stream of a recent high-resolution system. Even if this problem can be solved, the ASIC does not support ROI compression nor does it provide glue logic for the interface to the detector or for house keeping. The ASIC provides limited support for radiometric corrections, but, if this functionality is not or cannot be used, a further processing device is needed.

In general, there is the problem that present components-off-the-shelf (COTS) technology usually cannot be used for space applications. Nevertheless, a qualification can show that a certain component can be used - perhaps with small modifications [112]. Today's commercial CPUs, GPUs or ASICs are not radiation-tolerant, are not sufficiently reliable, are too slow, have high power consumption, or are simply not qualified for space 


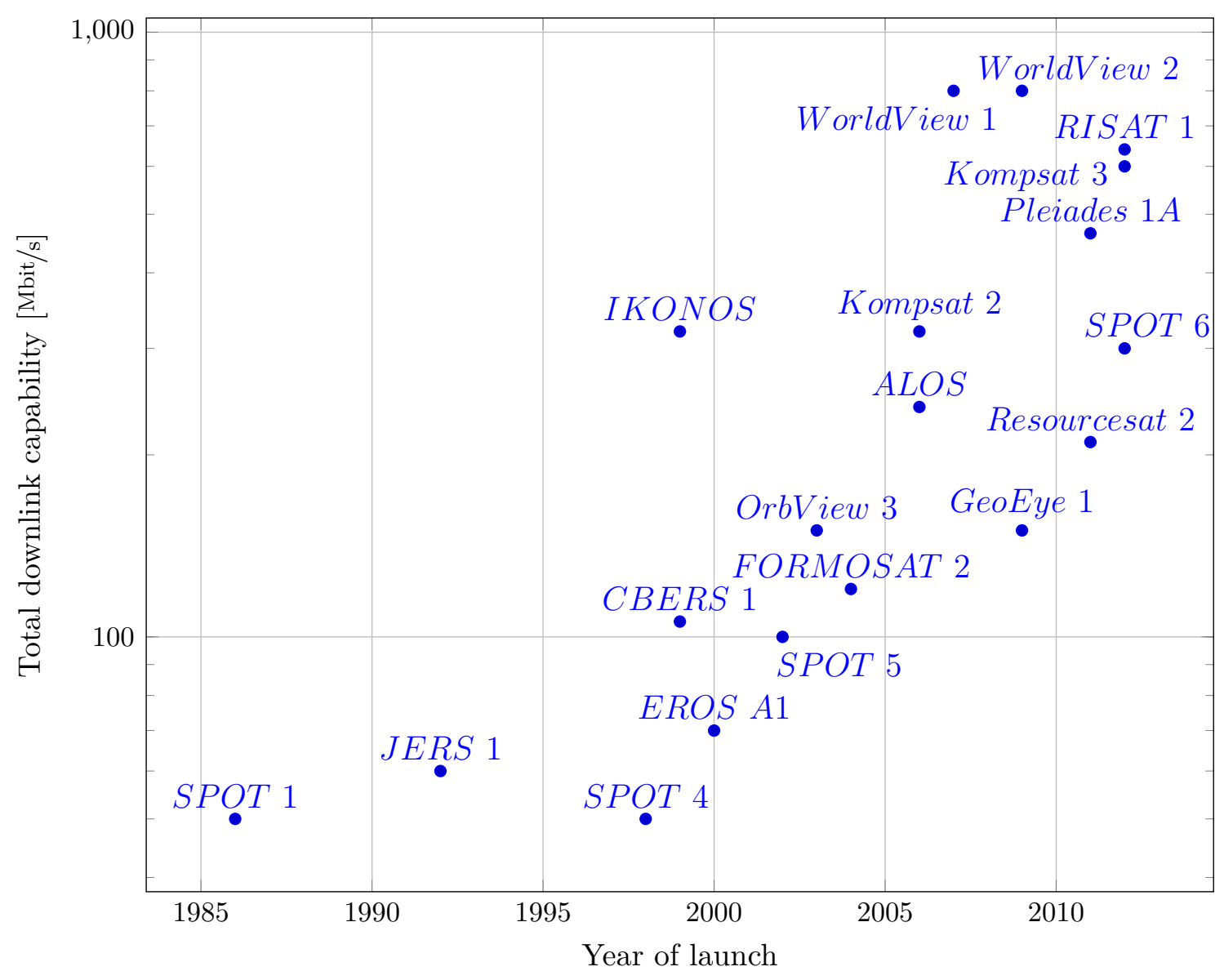

Figure 1.7: The downlink capability of some outstanding civil remote sensing satellites.

usage. For these reasons, radiation-hardened ASICs and FPGAs are used increasingly for on-board processing.

In summary, there is currently no integrated solution for high-resolution image data compression that can be used directly on an FPA. Even if the functionality is covered in most parts, many devices leads to complex designs and high costs. A reconfigurable architecture with a high data throughput and with an extended functionality is needed to integrate the image data compression onto the FPA.

\section{Future systems}

It can be assumed, that the data rate will increase continuously, quadratically with the GSD, and the downlink capability will remain limited with respect to the data rate. For future application scenarios, a fast and flexible access to the compressed data is necessary to serve different requests of different ground stations. As an improvement of the currently used store-and-download strategy, i. e. first storing the images and subsequently sending them to the ground station, it is desirable to have much more flexibility on the decision 


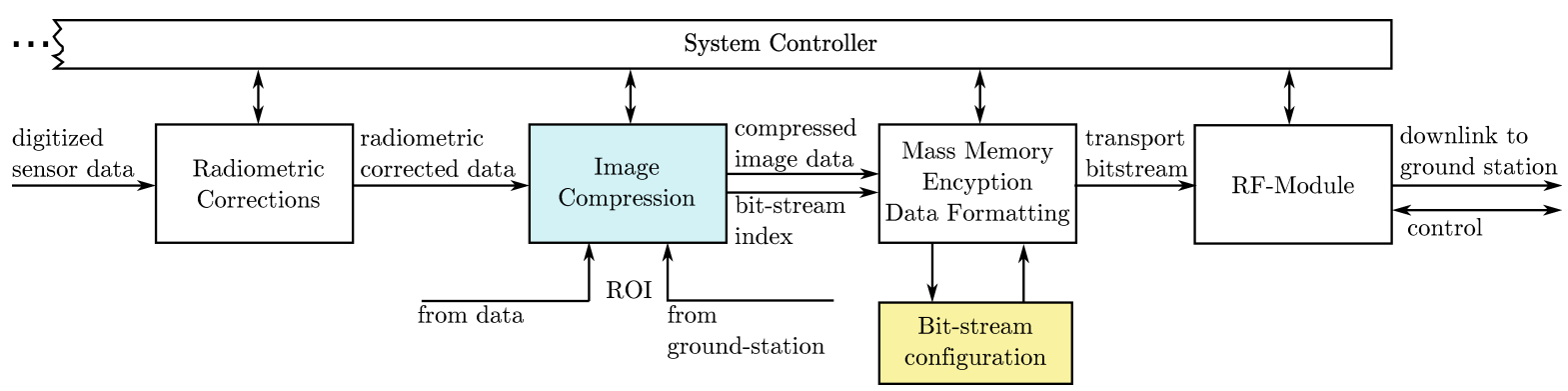

Figure 1.8: Concept of a new satellite image compression system.

what to record and on the generation of the end user data product. In the future, it will be possible to derive real-time application-specific information from the image on-board the satellite also with high-resolution images. In any conceivable scenario, data must be processed automatically, reliably, and fast.

ROI coding is useful in scenarios where on-board classification, registration or object or change detection algorithms are used. If a certain event is detected or a matching object is found, the compression system might encode the corresponding area with a higher detail or lossless. If there are multiple ground stations with divergent downlink capability, a re-assembling of already compressed image data might be desirable in order to adjust the amount of data to the bandwidth of the transmission channel. Another application scenario for this approach are ground stations with different access rights to the resolution level or spatial areas of the images.

\subsection{Contribution}

The thesis presents a new architecture for wavelet-based image data compression that can be used on reconfigurable hardware available for space applications. Figure 1.8 shows the principles of a system that uses this architecture. It is characterized by the fact that

- it uses a new approach for ROI coding and allows using online segmentation algorithms,

- it offers scalability of the compressed image data that allows a flexible access to spatial and spectral data,

- it supports high-resolution multi-spectral compression,

- it can be adapted to various mission requirements,

- it is developed for space-qualified reconfigurable hardware,

- and it outperforms all of the previously published approaches in terms of data throughput. 
ROIs can be coded with different quality parameters in order to reduce the memory demands of the compressed bit-stream. The new hardware architecture will allow the use of online segmentation algorithms that can be used for ROI extraction, e. g. change or event detection.

Scalability of the compressed image data can shorten the time until an image product is ready for the customer: On request, stored images can be re-assembled according the application's needs as requested by the ground station. This process will be called bit-stream configuration. The compressed image data shall be sent to the storage unit in a way, that

- Overview images can be transmitted in a lower spatial or spectral resolution,

- Spatial regions can be transmitted in higher resolution (spatial ROI) and

- Spectral information can be sent in higher resolution (more spectral channels; spectral ROI).

To achieve this, parts of the compressed image data should be extracted without re-coding. This concept of interactive transmission and decoding is similar to Google Earth and is also provided by JPEG2000 Interactive Protocol (JPIP) [55]. If a ROI is available during compression, it should also be possible to irreversible remove unnecessary information and to extract relevant information.

Beside spatial correlation, images obtained from air-borne and space-borne multi-spectral (MS) or hyper-spectral (HS) cameras also exhibit a high degree of spectral correlation. To achieve an optimal compression ratio, these correlation must be properly exploited. The concept of scalability can be combined with a spectral decorrelation technique in order to achieve multi-spectral compression.

There is no such technology which offers ROI support and post-compression bit-stream configuration. Commercial implementations such as the JPEG2000 Codec from Analog Devices cannot be used for space applications because of radiation issues [10]. The Airbus Defence and Space CWICOM ASIC [34] is radiation hardened, but does also not provide ROI support or bit-stream flexibility. An own implementation based on a field-programmable gate array (FPGA) platform was chosen, because there are radiation hardened FPGAs $[81,128]$ and this solution provides the highest degree in flexibility and expected data-rates. The requirements, a design, and an implementation based on reconfigurable hardware is presented. Furthermore, it can be perfectly integrated directly onto an FPA, since FPAs usually already integrate an FPGA. For comparative purposes, however, studies of central processing unit (CPU) and graphics processing unit (GPU) implementations were performed.

A method for image compression is selected and adapted. It must be possible to operate in lossless as well as in lossy and near-lossless compression mode and to control 
the distortion in terms of compression ratio. Furthermore, it shall be able to compress images with a high dynamic range and a high data rate at the selected platform. To obtain a high re-usability in future projects, the design should be as general as possible.

To the best of our knowledge, the proposed architecture outperforms all of the previously published approaches in terms of data throughput. An implementation of the architecture was compared with several recent approaches [10, 34, 70, 71, 126].

The secondary goal of this thesis is derive requirements on an on-board computer as mass storage controller and data formatter to communicate with ground station.

\subsection{Organization}

This thesis is structured as follows: In Chapter 2, the fundamentals of optical satellite imagery, image data compression on-board a spacecraft, and the CCSDS 122.0-B-1 image data compression standard are presented. CCSDS 122.0-B-1 is the basis for the extensions presented in Chapter 3. In Chapter 4, a new architecture for image data compression on-board satellites is presented. In Chapter 5, experiments and their results are presented and discussed. Finally, conclusions are drawn in Chapter 6 and an outlook is given. 


\section{Fundamentals and Related Work}

This chapter presents fundamentals and related work required for this thesis. It is structured as follows: In Section 2.1, the fundamentals of optical remote sensing and satellite imaging are presented. The basics of wavelet-based image data compression are presented in Section 2.2. FPGA technology is chosen as the basis for an implementation. Thus, some architectures for discrete wavelet transform (DWT) and on-board image data compression are presented in Section 2.3. The CCSDS 122.0-B-1 compression standard is presented in Section 2.4. A short summary of this chapter is given in Section 2.5.

\subsection{Optical Remote Sensing}

In remote sensing, information about the Earth's surface or other objects that are not directly accessible is acquired without physical contact. Reflected or emitted radiation forms a carrier of information that is measured and interpreted by a detector. Basically, one can distinguish between active and passive sensor systems. Passive sensor systems gather radiation that is reflected or emitted by objects on the Earth's surface. Active sensor systems emit energy in order to scan objects and areas whereupon a sensor then detects and measures the radiation that is reflected or back-scattered from the target (e. g. radar and lidar).

In optical remote sensing, optical sensor systems are used. Figure 2.1 shows the process of optical remote sensing using a passive sensor system. The process starts with sunlight illuminating objects on Earth. Different objects or materials reflect or absorb differently at certain wavelengths. The reflected part of the incident radiation or radiation from light-emitting objects enter the optics of the imaging system. At the end of the process, image data is sent to the ground station, where it is further processed.

\subsubsection{Optical Sensor Systems}

A typical optical sensor system as shown in Figure 2.2 consists of several components: The reflected part of the incident radiation or radiation from light-emitting objects enter the optics of the imaging system. The optics images the light beam on the detector using transmission or refraction. The detector converts photons into electrons and generates an electron flux which in turn leads to an analog signal. The analog-to-digital converter (ADC) converts the samples of the analog signal into a digital number (DN). Optionally, radiometric corrections are applied in a pre-processing stage in order to calibrate the detector's response. An image compression module that works either online or offline, 


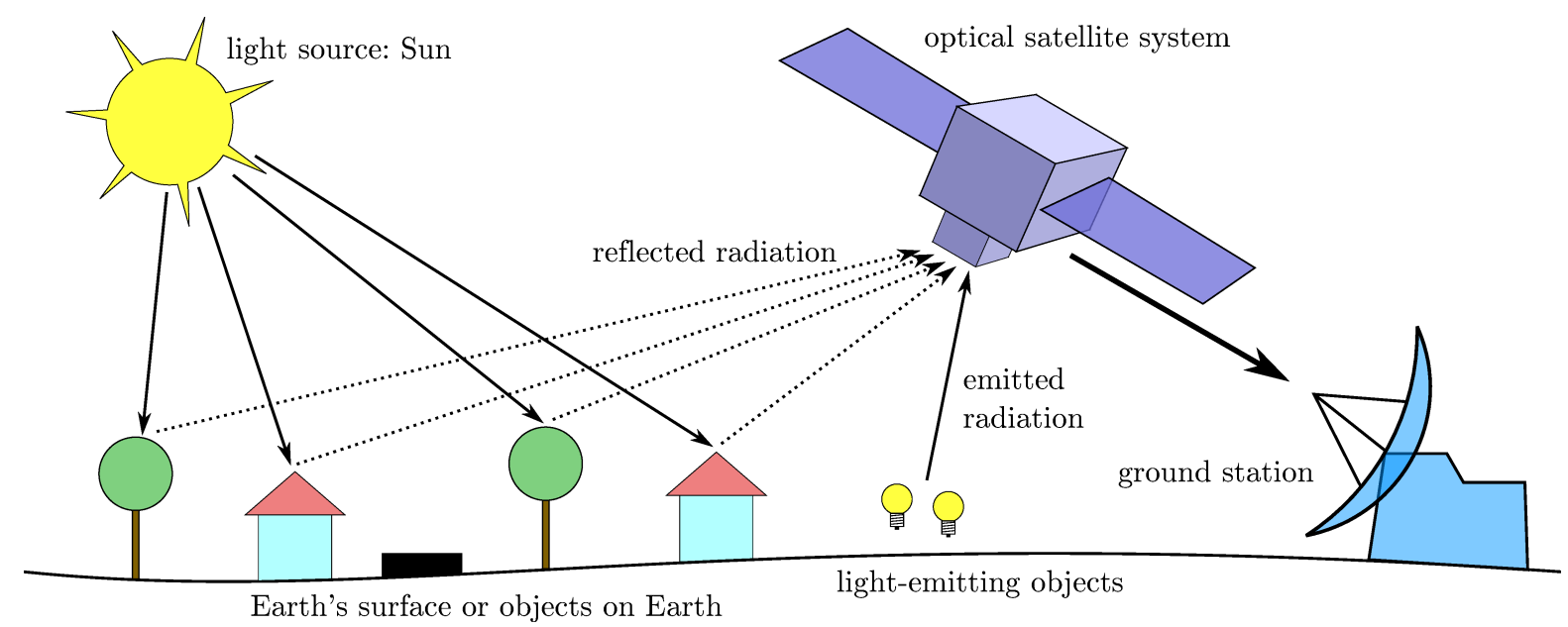

Figure 2.1: Optical remote sensing using a passive sensor system.

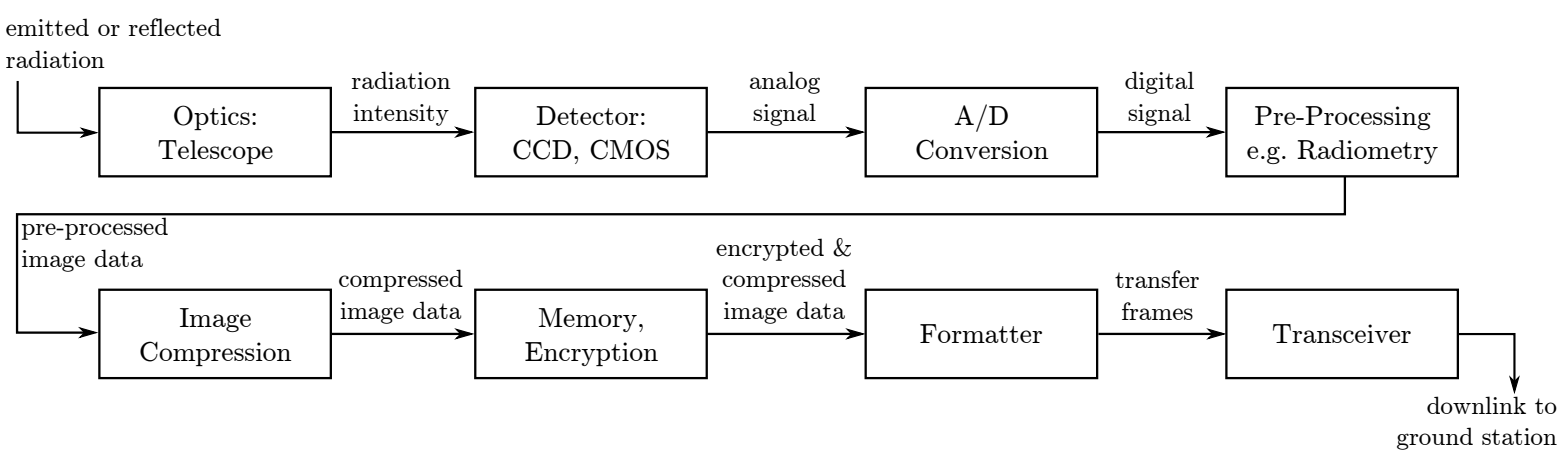

Figure 2.2: Schematic of an optical sensor system on-board a space craft.

is often used in an imaging satellite. Online means that the compression is applied in real-time before storing the data. Offline means that the image data is stored before compression. If the ground station requests image data, it will be read from memory, encrypted, formatted into transfer frames, and transmitted.

\subsubsection{Components}

Optics The optics (telescope) of an imaging system consists of one or more lenses and/or mirrors. A comprehensive overview of the topic is given in [47]. The thin lens model shown in Figure 2.3 is often used to describe the formation of an image on the detector. It can be used as an approximation for the explanation of the relationship between GSD, i. e. the distance between pixel centers projected on the ground $(\equiv B)$, altitude $h(\equiv g)$, focal length $f$, and pixel pitch $\Delta p_{x}(\equiv G)$.

A thin lens has a small thickness and a small curvature of the boundary surfaces. Each ray from a point arising on the lens parallel to the optical axis is refracted and proceeds through the focal point $F$. On the other side, any ray from point arising through frontside "focal point" $F^{\prime}$ is also refracted and proceeds parallel to the optical axis. The chief 


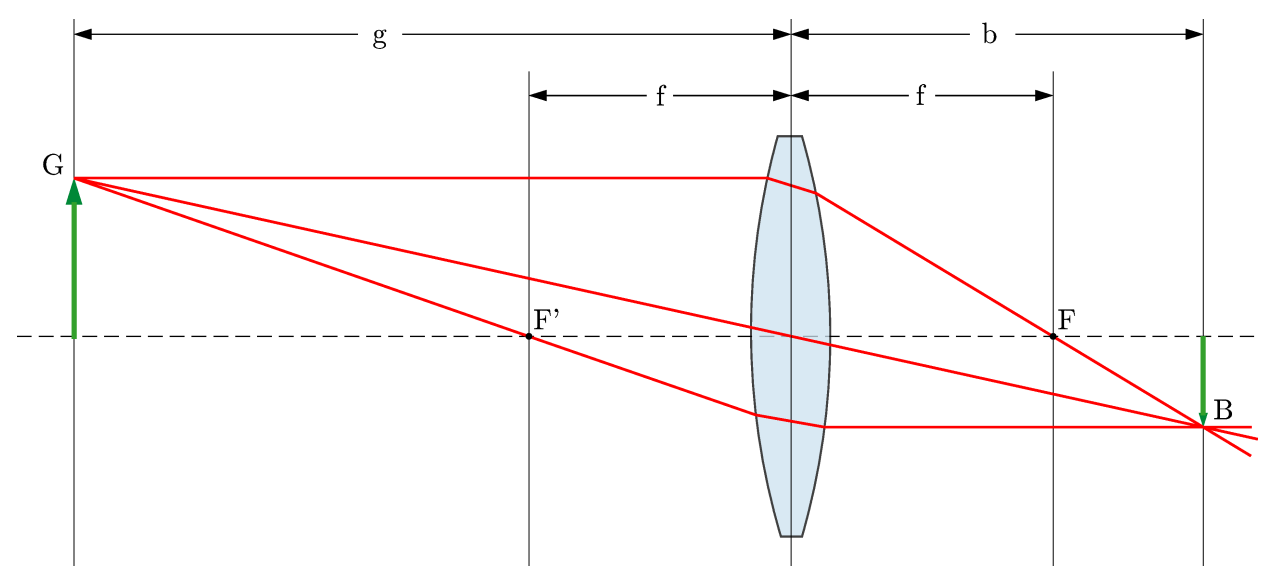

Figure 2.3: Fundamentals of imaging by a thin lens [122].

rays, i. e. the rays arising at the center of the lens, do not change their direction. All rays from an object $G$ passing the lens are combined in point $B$. The thin lens formula is used to describe this relationship:

$$
\frac{1}{f}=\frac{1}{g}+\frac{1}{b}
$$

where $f$ is the focal length, $g$ is the object distance, and $b$ is the image distance. If this equation is violated, the object point $G$ imaged on the image plane is out of focus. The thin lens formula is used for finite imaging distances with $g \ll \infty$. The intercept theorem provides the following equation:

$$
\frac{B}{G}=\frac{b}{g}
$$

Assuming an optical system with focal length $f=12 \mathrm{~m}$, altitude $h=600 \mathrm{~km}$, and pixel pitch $\Delta p_{x}=10 \mu \mathrm{m}$, the GSD can be determined as follows:

$$
\begin{aligned}
G S D & =\frac{h \Delta p_{x} \cdot(h-f)}{h f}=\frac{\Delta p_{x} \cdot(h-f)}{f}=\frac{\Delta p_{x} h}{f}-\Delta p_{x} \\
& =\frac{10 \mu \mathrm{m} \cdot 600 \mathrm{~km}}{12 \mathrm{~m}}-10 \mu \mathrm{m}=0.5 \mathrm{~m}-10 \mu \mathrm{m} \\
& \approx 0.5 \mathrm{~m}
\end{aligned}
$$

For this example, the distance $b-f$, i. e. the distance between the focal point $F$ and the image plane, is just approximately $240 \mu \mathrm{m}$. If the object distance $g$ rises, the corresponding term in the thin lens formula becomes negligible small. One speaks of "imaging from infinity". This approach is often applicable for imaging satellites and is shown in Figure 2.4. Equation 2.1 can be rewritten as follows:

$$
\frac{1}{f}=\frac{1}{\infty}+\frac{1}{b} \Longrightarrow \frac{1}{f}=\frac{1}{b}
$$

The consequence of this situation is that the focal point $F$ is located in the image plane. 


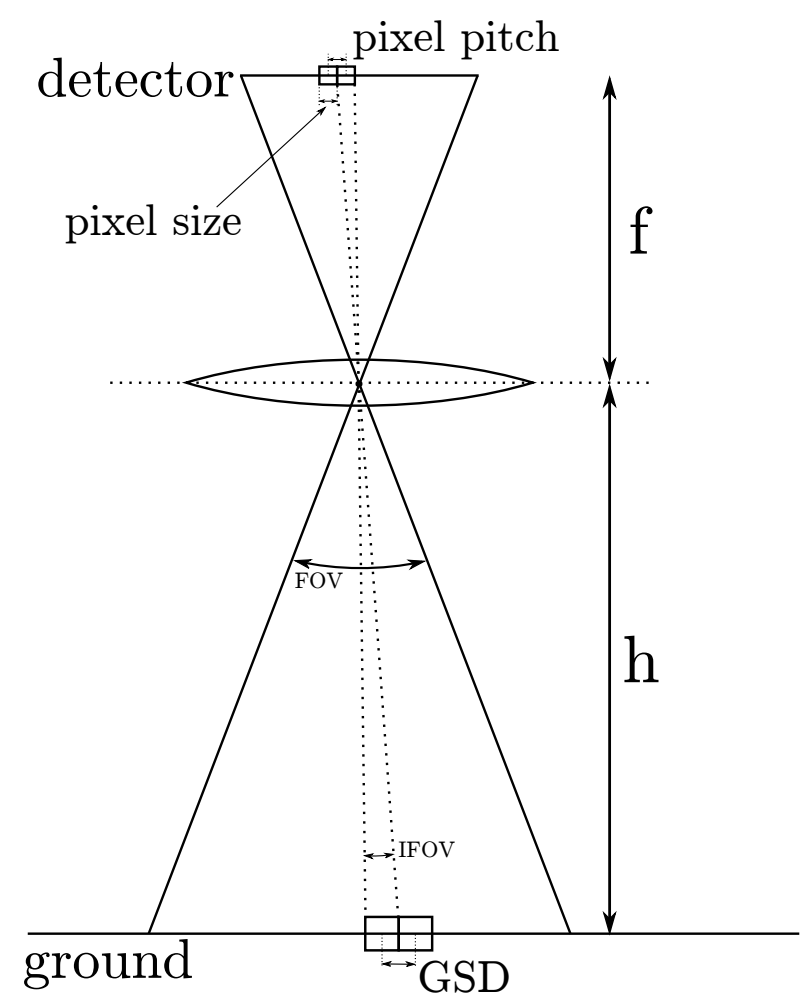

Figure 2.4: Imaging situation for an optical satellite: IFOV, GSD, pixel pitch and pixel size.

Detector A detector converts a physical or chemical quantity into an electronic signal. A photon detector generates electrons via the photoelectric effect: If the photon energy $E_{\lambda}$ of the wavelength $\lambda_{\text {cutoff }}$ is greater than the band gap $E_{\text {bandgap }}$ of the semiconductor ${ }^{1}$, one or more electrons excites from the valence band to the conduction band thus creating electron-hole pairs.

$$
E_{\lambda}=\frac{h c}{\lambda}>E_{\text {bandgap }}
$$

where $h$ is Planck's constant and $c$ is the speed of light. The electron-hole pairs diffuse through the semiconductor lattice structure, thus generating an electrical current or voltage proportional to the intensity of the incident radiation. The sensitivity of a photon detector can be described with the quantum efficiency $(\mathrm{QE})$ which is percentage of incident photons that produce charge carriers as a function of the wavelength $\lambda$. Current silicon detectors are based on the $\mathrm{CCD}^{2}$ or $\mathrm{CMOS}^{3}$ technology. While CCD refers to a fundamental method for charge transfer, CMOS refers to a technology for constructing integrated circuits.

A CMOS detector is - as the name suggests - produced in CMOS technology. In a CMOS detector, it is possible to implement pre-processing and control functions directly

\footnotetext{
${ }^{1}$ For silicon: $E_{\text {bandgap,SI }}=1.14 \mathrm{eV}$

${ }^{2}$ charge-coupled device (CCD)

${ }^{3}$ complementary metal-oxide semiconductor (CMOS)
} 


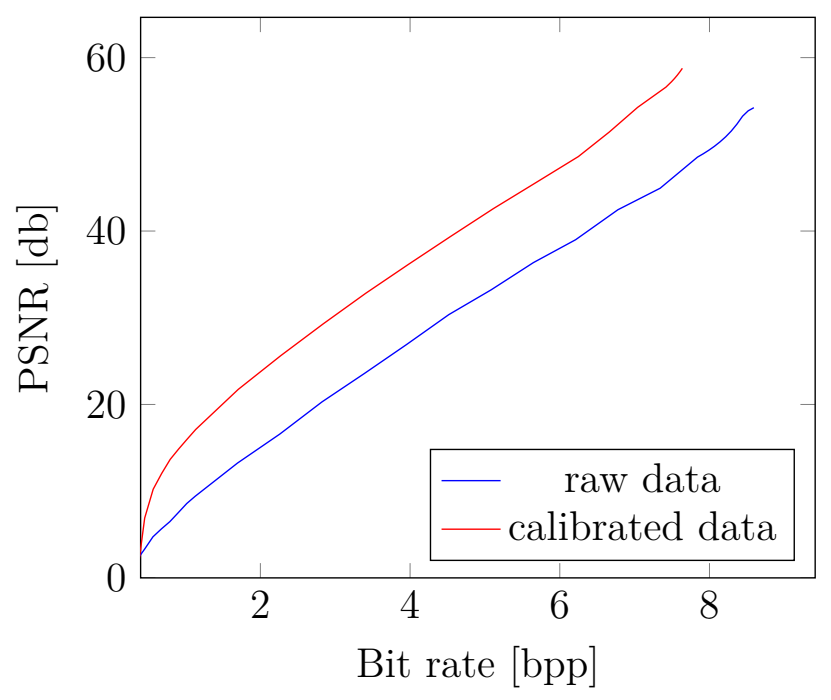

Figure 2.5: Rate-distortion curve of raw (aviris_sc0_raw) and radiometric corrected data (aviris_sc0_cal). The curves were generated with a reference implementation of the CCSDS 122.0-B-1 standard. The reference implementation was developed for this thesis and also includes extensions for multi-spectral compression.

on the chip. A CMOS detector can have a separate amplifier for each pixel that converts the charge to a voltage, and usually has a matrix that allow to address and read out each pixel individually.

Data pre-processing If an optical sensor system uses on-board image compression, data pre-processing is often performed to improve the compression ratio. In the preprocessing stage, corrections are applied based on the calibration of the instrument. Calibration is the process of determining the relationship between the expected and the actual measurement of an instrument. Camera calibration is inevitable to derive accurate physical measurements from camera images. The modeling of object and image point projection is called geometric calibration whereas radiometric calibration comprises the modeling of the detector's response to an incident light beam.

Although applying geometric corrections is indispensable for geo-referencing of the acquired image data, it is usually not performed on-board a spacecraft before compression. On the one hand geometric correction is not likely to improve the compression ratio, and on the other hand, the implementational effort to perform geometric corrections on-board the satellite is relatively high. In addition, it is not generally reversible through the use of re-sampling and any mistake can mean that the image data is unusable. An accuracy analysis for stereo processing with generic camera projection models, with a main aspect on radially induced errors was presented in [91]. 
In contrast to geometric normalization, applying radiometric normalization before compression can significantly improve the compression ratio. Figure 2.5 shows a rate-distortion curve of raw and radiometric normalized data.

\subsubsection{Parameters}

On the way from the light source to the image, several components such as the environment, the lenses, the detector, and the electronics of the sensor system introduce some amount of distortion. The term image quality is a measure to characterize the imaging performance of an optical system. Image quality is influenced by properties of the scene and by the components of a system and can, for example, be quantified by signal-to-noise ratio (SNR) and modulation transfer function (MTF). The MTF is a measure for the spatial quality of an image, while the SNR is a measure for the radiometric quality of an image. The term resolution is used to describe the ability of a device to separate values of a physical quantity.

Signal-to-noise ratio In optical systems, SNR is used to describe the radiometric properties of the system. SNR is a measure that gives the relationship of the signal, usually containing the information, to the noise. It is defined as the power ratio between a signal and the noise. The SNR can, for example, be increased by using a longer integration time. This in turn has an influence on the MTF of the system (see below).

Modulation transfer function Effects like diffraction, aberration, movement of the detector, and properties of the detector itself lead to a spreading of a single object point. The MTF describes the loss of contrast at the edges of an object relative to the contrast of its pictorial representation. The amount of spreading is a measure for the quality of an imaging system. This impulse response of an imaging system is described by the point spread function (PSF). Figure 2.6 shows the process of image formation by convolution of the real light source with a PSF.

For a linear system, the individual MTFs of the system's components can be combined in order to create the overall system MTF. The overall MTF of the imaging system consisting of components $a, b$, and $c$ is as follows:

$$
\mathrm{MTF}_{\text {overall }}=\mathrm{MTF}_{\mathrm{a}} \cdot \mathrm{MTF}_{\mathrm{b}} \cdot \mathrm{MTF}_{\mathrm{c}}
$$

Depending on the definition of the overall system, these components might be the optics, the detector, the atmosphere or the platform stability.

Methods for MTF determination are discussed in [13]. A method often used is based on edge targets: The edge spread function (ESF) is the systems response to a high dark-bright contrast edge. With the slanted edge algorithm used in [63] it is possible to create a super resolution version of the ESF. The derivative of the ESF is the line spread function (LSF). The LSF is then normalized to 1. The Fourier transform of the LSF 


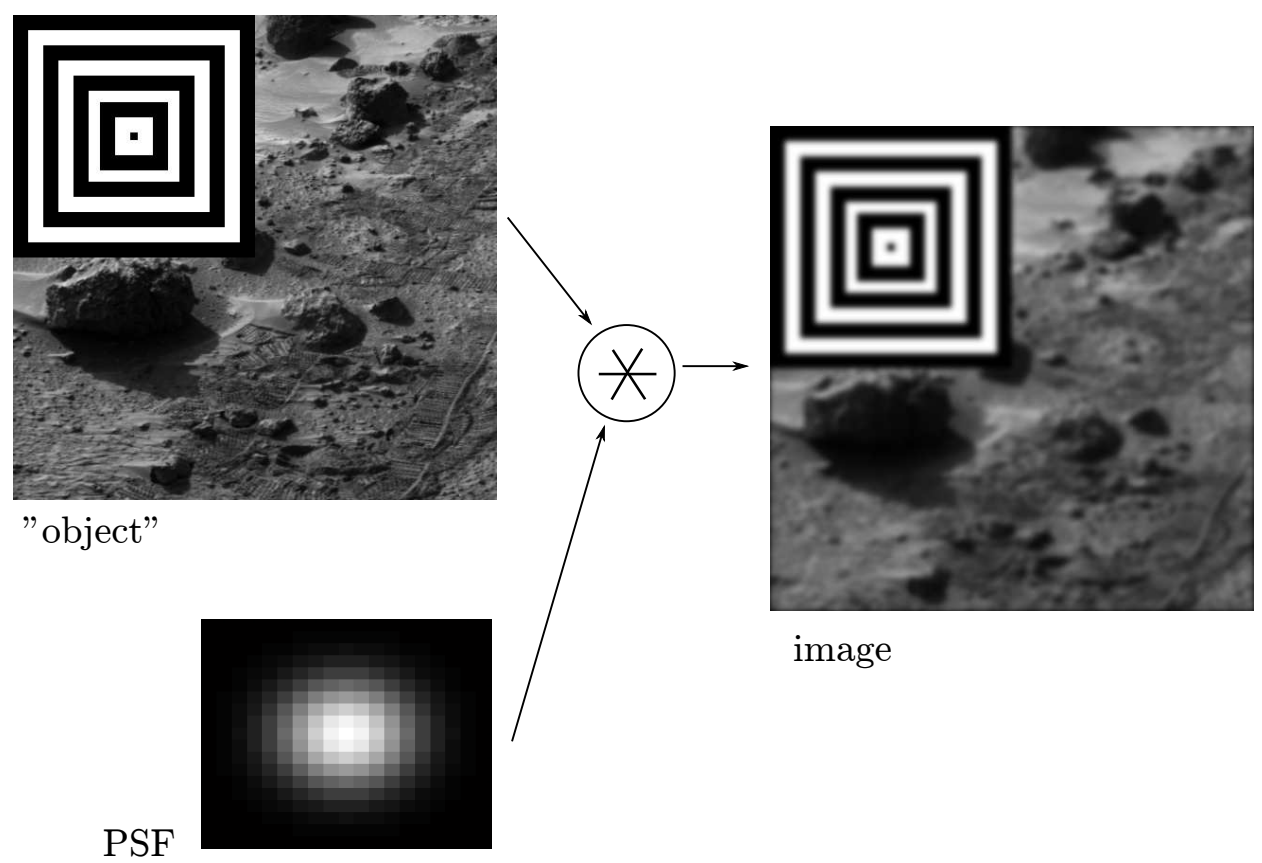

Figure 2.6: Convolution of an "object" and a PSF.

gives the MTF. A toolbox for determination of the MTF with the slanted-edge approach is available in [17].

Resolution A distinction is made between spatial, spectral, temporal, and radiometric resolution. One goal of remote sensing is often to detect even small changes in these domains. Thus, resolution is an essential characteristic of remote sensing sensors and critical for the usability of the data for different applications.

The Rayleigh criterion ${ }^{4}$ can be used to estimate the spatial or spectral resolution of an optical system. It states that "Two point sources are regarded as just resolved when the principal diffraction maximum of one image coincides with the first minimum of the other." [121]. Figure 2.7 shows this relationship for two point sources. Commonly used terms to quantify the spatial resolution are instantaneous field of view (IFOV) and GSD. The IFOV is the angular range $\alpha$ from which the reflected radiation can be collected by a single pixel detector. The IFOV of a pixel on the optical axis is as follows:

$$
\alpha=\frac{p_{x}}{f}, \quad p_{x} \ll f
$$

where $p_{x}$ is the detector's across-track pixel pitch. For an array detector, the along-track pixel pitch $p_{y}$ can be defined. For quadratic pixels, the following applies: $p_{x}=p_{y}$. For push-broom detectors, a distinction can be made between across-track and along-track: The across-track GSD is defined as above whereas the along-track GSD depends on the

\footnotetext{
${ }^{4}$ Invented by John William Strutt, 3rd Baron Rayleigh (1842-1919).
} 


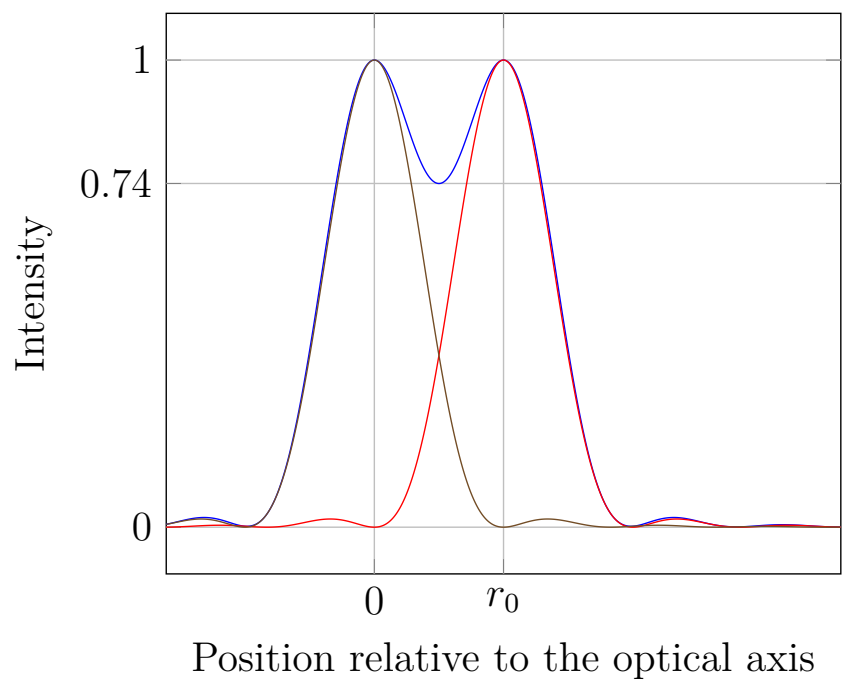

Figure 2.7: Rayleigh criterion for two point sources, simplified for the one-dimensional case.

sampling interval. As shown in Figure 2.4, the spatial resolution of a system can be characterized by its detector pixel pitch $\Delta p_{x}$, the focal length $f$, and the altitude of the sensor $h$.

Spectral resolution describes the ability of a sensor system to separate individual wavelength ranges. According to the Rayleigh criterion, two spectral lines of approximately the same intensity are dissolved when the intensity between the maxima of both lines has fallen to less than $80 \%$. Panchromatic sensors use only a single spectral channel with a wavelength in the range of $450-900 \mathrm{~nm}$. Multi-spectral sensors combine several spectral channels (typically $3-5$ ) in the visible region. Hyper-spectral sensor typically have several hundred spectral channels $[56,57]$.

The temporal resolution of a sensor corresponds to the temporal distance between two images of the same area. Images sequences with a high temporal resolution or repetition rate are particularly suitable for the documentation of dynamic processes.

Radiometric resolution is a measure for the smallest, still distinguishable electromagnetic radiation of a remote sensing sensor. Radiometric resolution provides information on the number of gray level values that can be detected by the system in a particular spectral channel. The relationship is often referred to as dynamic range.

\subsubsection{Satellite Imaging}

Most imaging satellites orbit the Earth in a sun-synchronous orbit (SSO) at an altitude between $400 \mathrm{~km}$ and $900 \mathrm{~km}$. A satellite in a SSO ascends or descends over any given Earth latitude at the same local mean solar time. The angle with the equator is called inclination. An inclination of zero indicates an equatorial orbit whereas an inclination of $90^{\circ}$ indicates 


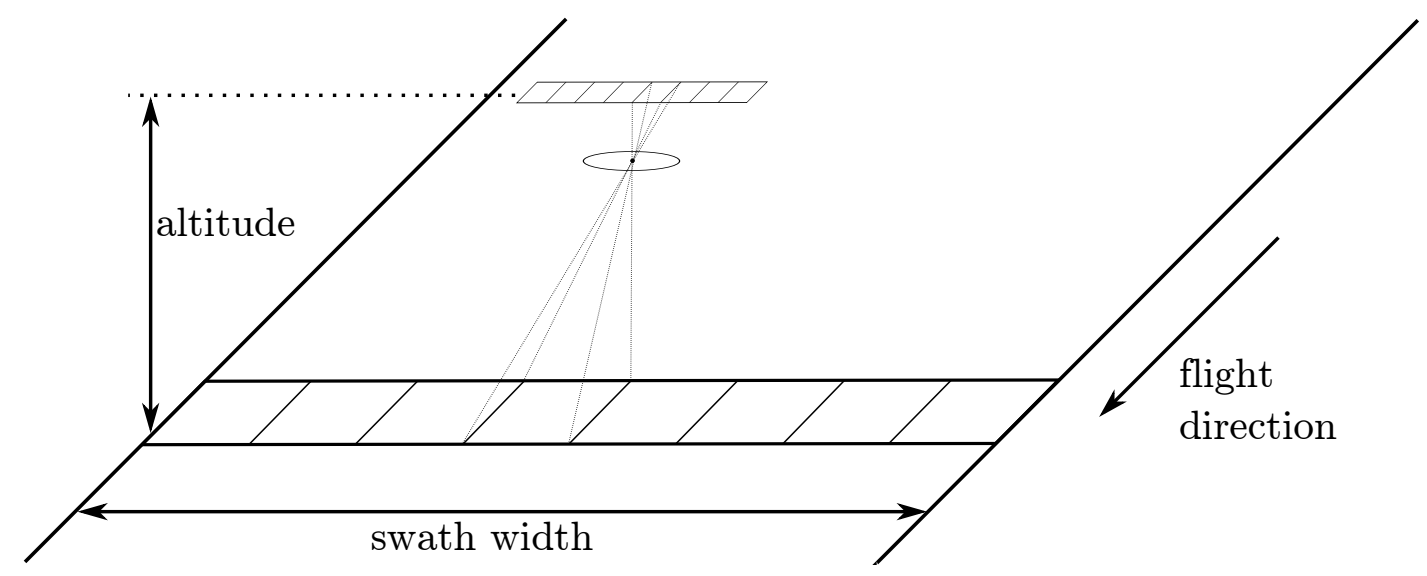

Figure 2.8: Principle terms of satellite imagery.

a polar orbit. Imaging satellites typically have an inclination of approximately $98^{\circ}$ to cover almost the entire Earth surface. A typical period time for a complete orbit is 96 minutes so that such a satellite orbits the Earth approximately fifteen times a day.

The swath refers to the stripe of the Earth's surface from which the image data is acquired. The swath width is the width of this stripe. The orbital speed of a satellite is the speed at which it orbits the center of mass of the Earth. Since the mass of the satellite is negligible compared to the mass of the Earth, the speed of a satellite is as follows:

$$
v_{0} \approx \sqrt{\frac{G M}{r}}
$$

where $G$ denotes the gravitational constant ${ }^{5}, M$ denotes the mass of $\operatorname{Earth}^{6}$, and $r$ denotes the radius of the satellite orbit ${ }^{7}$. KOMPSat-3 has an altitude of $685 \mathrm{~km}$ so that the orbital speed of the satellite is $7.516 \mathrm{~km} / \mathrm{s}$ [35]. Figure 2.10 shows the orbital speed of a satellite in SSO as a function of the altitude. The ground track of a satellite is the path of the satellite projected on the surface of Earth. The ground track speed of an geo-synchronous satellite is approximately $7 \mathrm{~km} / \mathrm{s}$ :

$$
v_{\text {ground }}=\frac{v_{0} \cdot R}{r}=\frac{v_{0} \cdot R}{R+h}
$$

where $R$ denotes the radius of Earth. When imaging in nadir mode, i. e. perpendicular to surface of Earth, the revisit time of a satellite is pretty high so that recent imaging satellites have a steerable platform. This allows viewing off-nadir and to rotate the satellite around any axis through the use of reaction wheels [124]. WorldView-2 has a revisit time of 1.1 days at $1 \mathrm{~m}$ GSD.

\footnotetext{
${ }^{5} G=6.67 \cdot 10^{-11} \mathrm{~N}(\mathrm{~m} / \mathrm{kg})^{2}$

${ }^{6} M=5.97219 \cdot 10^{24} \mathrm{~kg}$

${ }^{7}$ Satellite's altitude $h$ plus Earth radius $R=6371 \mathrm{~km}, r=R+h$
} 


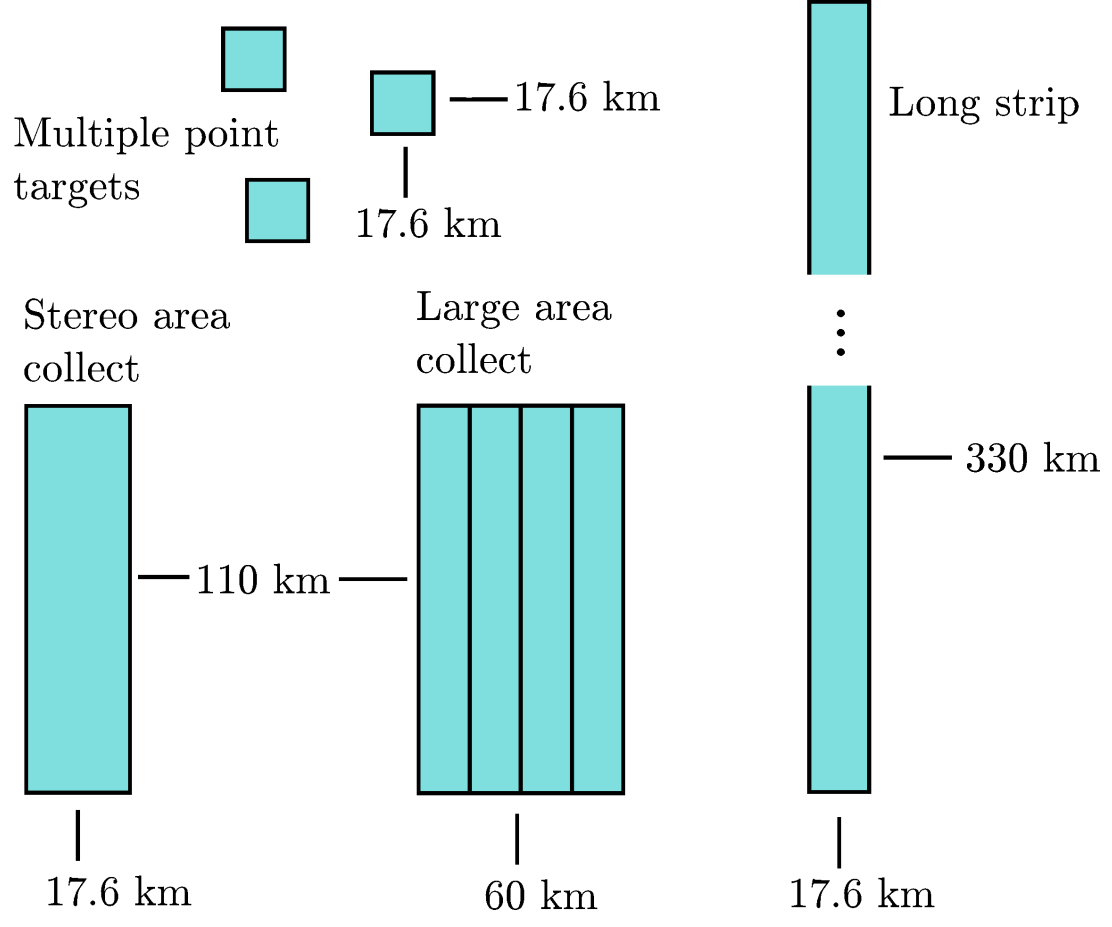

Figure 2.9: Example of image collection scenarios with a single-sensor concept [96].

There are other modes for image acquisition: As already mentioned, a steerable platform enables the satellite to acquire images off-nadir. This results in a lower revisit time, but in turn, decreases the image quality that results in a lower spatial resolution. In nadir mode, WorldView-2 has a GSD of $46 \mathrm{~cm}$ whereas the GSD is $52 \mathrm{~cm}$ at $20^{\circ}$ off-nadir. The revisit time at $20^{\circ}$ off-nadir is 3.7 days. If the satellite has a steerable platform, forward motion compensation (FMC) can be used to decrease the ground track speed of the satellite. A lower ground track speed results in a higher dwell time $t_{\text {dwell }}$ which represents an upper limit on integration time $t_{\text {int }}$ :

$$
t_{\text {dwell }}=\frac{G S D}{v_{\text {ground }}} \geq t_{\text {int }}
$$

$t_{\text {int }}$ is usually lower than $t_{\text {dwell }}$ because some time is required for the setup and readout of the detector. The line-rate is the number of lines per second that are generated by a push-broom detector. Since the angle to the Earth's surface changes continuously in FMC mode, acquisition must be stopped if the angle exceeds a certain threshold. Thus, FMC limits the maximum size of an acquired image. Furthermore, the collection capacity decreases, i. e. the maximum area the satellite covers in a certain time. FMC also allows acquiring stereo image pairs from the same orbit and multiple point targets (see Figure 2.9). 


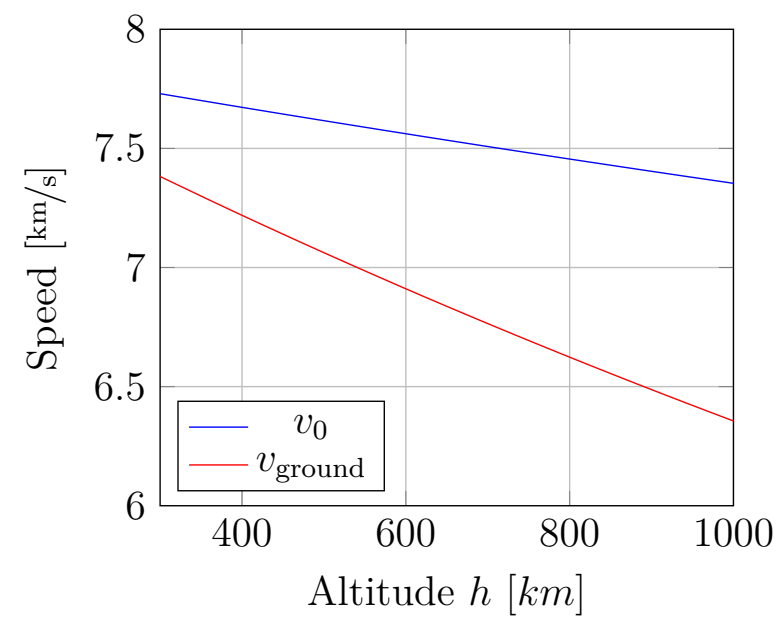

Figure 2.10: Orbital and ground track speed (nadir) of a satellite as a function of the altitude.

\subsection{Image Data Compression}

The aim of image data compression is to reduce the amount of memory or bandwidth needed to store and transmit images. It can be motivated by a bandwidth-limited transmission channel, the need of reducing the transmission effort, limited time for transmission, high costs for transmission, or limited storage capacity. Data compression in general is possible because information often contains redundancy or irrelevant information ${ }^{8}$. Lossless compression is achieved if the original information can be exactly reconstructed without a deviation. It is rarely possible to obtain lossless compressions ratios beyond 2:1. Higher compression ratios can be obtained if some error, which cannot be perceived or can be tolerated, is allowed. Lossy compression is usually achieved by removing irrelevant information. If the loss of information is negligible with respect to the observer or the application, it is called near lossless compression.

\subsubsection{Basic Concepts}

The structure of a typical image data compression chain is shown in Figure 2.11. A transform encoder consists of a decorrelation stage or forward transform in order to remove spatial or spectral redundancy, in case of lossy compression followed by quantization and, finally, coding to remove statistic redundancy. The decoding process is applied in reverse order with some approximations in the reconstruction stage.

\subsubsection{Information Theory}

A fundamental term of image data compression is the entropy ${ }^{9}$. It is derived from thermodynamics and is a measure for the disorder of a thermodynamic system. Its value

\footnotetext{
${ }^{8}$ E. g. image sequences often contain similar, consecutive images

${ }^{9}$ In 1948, it was introduced by the American mathematician Claude Elwood Shannon in the concept of information theory [101]
} 


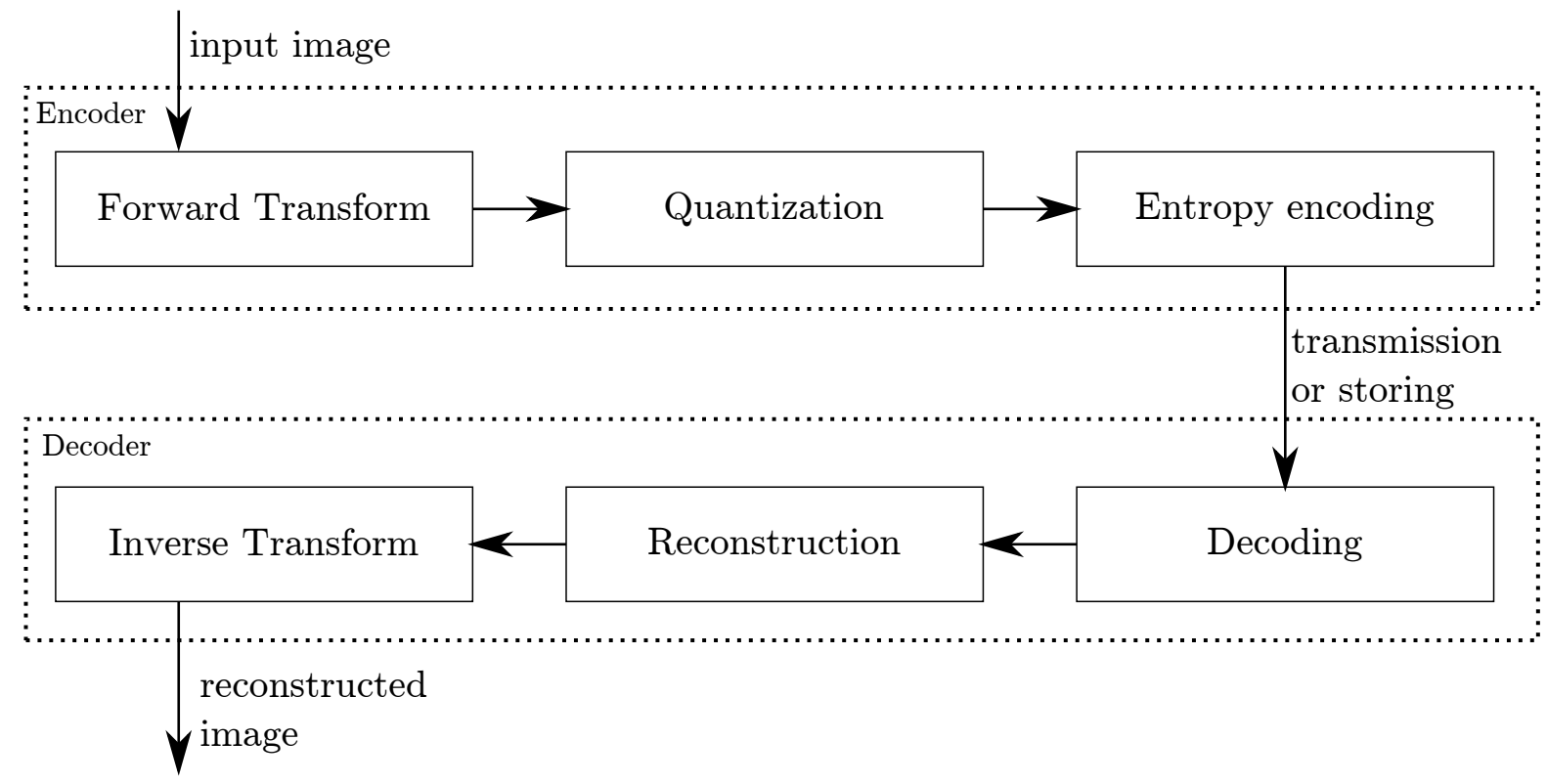

Figure 2.11: The symmetrical structure of a transform encoder and decoder.

can either remain constant for reversible processes or increase for irreversible processes, but never decrease (2nd law of thermodynamics).

A discrete-time signal $x[n]$ can be expressed as a finite series of symbols from a set $\left\{s_{i}\right\}$ where $i=1,2, \ldots, K$. A probability of occurrence $p_{i}$ can be assigned to each event. If the events are statistically independent, the entropy $H_{\text {src }}$ can be calculated as follows:

$$
H_{\mathrm{src}}=\sum_{i=1}^{K} p_{i} \log _{2} \frac{1}{p_{i}}=-\sum_{i=1}^{K} p_{i} \log _{2} p_{i} \quad[\mathrm{bit} / \mathrm{symbol}]
$$

The entropy has its maximum value, if the symbols are uniformly distributed $\left(p_{i}=1 / K\right)$, and its minimum value if only one symbol occurs in the signal.

The average number of bits per symbol needed to code a signal is

$$
S_{\mathrm{src}}=\frac{N_{B}}{N_{A}} \geq H_{\mathrm{src}}
$$

where $N_{A}$ denotes the number of signal values, and $N_{B}$ denotes the number of bits needed to code the signal. If the entropy is maximal, the same (minimum) number of bits can be assigned for coding each symbol. Otherwise, it leads to coding redundancy:

$$
R_{\mathrm{cod}}=S_{\mathrm{src}}-H_{\mathrm{src}} \geq 0
$$

If the symbols are not statistically independent, then there is a certain correlation between the symbols. Thus, the "real" entropy of the signal is slightly lower than $H_{\text {src }}$. The conditional entropy $H_{\text {cond }}$ is used to describe the inter-symbol redundancy:

$$
R_{\text {is }}=H_{\text {src }}-H_{\text {cond }} \geq 0
$$


Coding redundancy and inter-symbol redundancy can be reduced by coding (see Section 2.2.1.3).

If the signal contains information that cannot be perceived by the recipient or those for which the recipient can accept a loss of information, then one speaks of irrelevance.

\subsubsection{Data Compression Techniques}

In principle, data compression techniques can be classified into three groups [107]:

Information reduction Removal of irrelevant information (lossy).

Decorrelation Removal of spectral-, spatial- or temporal-redundant information (lossy or lossless).

Coding Encode data to remove statistic redundancy (lossless).

Information Reduction Information reduction can be achieved by sub-sampling or quantization $^{10}$. In sub-sampling, the temporal or spatial resolution of a signal $x[n]$ is reduced by a factor of $M$ (Figure 2.12):

$$
y[m]=x[m \cdot M]
$$

Note that the bandwidth of the signal must be restricted in order not to violate the sampling theorem and to avoid aliasing effects. The combination of an anti-aliasing and sub-sampling is also called re-sampling. Quantization can be distinguished between scalar and vector quantization. In scalar quantization each signal value $x$ is mapped to a quantized symbol $q$ :

$$
\text { Quantization : } \quad x \mapsto q \quad(q \in \mathbb{Z}) .
$$

The reconstruction maps the quantized symbol $q$ to a reconstructed value $[x]_{Q}$ :

$$
\text { Reconstruction : } \quad q \mapsto x_{q}
$$

Since these are not bijective functions, quantization is usually not reversible. The quantization error is the difference between the signal value $x$ and the reconstructed value $x_{q}$ :

$$
e_{q}=x-x_{q}
$$

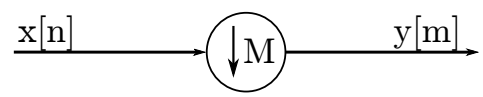

Figure 2.12: Sub-sampling of a signal $x[n]$ by a factor of $M$.

\footnotetext{
${ }^{10}$ High-level processing like change detection or feature extraction also reduce the amount of information. Although not considered in this thesis, these algorithms can be executed on a data processing unit after the image data compression unit for further information extraction.
} 
In vector quantization, the theory is the same, except that $x$ and $x_{q}$ are vectors. The quantization intervals can have an equal width (uniform quantization) or any other quantization function. In image compression, these are often adapted to a probability density function (PDF) of the signal to be quantized. They can also be perceptual- or SNR-optimized. More details and examples on scalar and vector quantization can be found in [107].

Decorrelation The aim of decorrelation is the compression of information, i.e. to reduce auto-correlation within a signal. According to [107], methods for decorrelation can be divided into three groups:

- Prediction of signal values from other (already known) values.

- Transform: Decomposition of a signal into basis functions.

- Filter bank: Decomposition of a signal into (overlapping) frequency ranges or components.

Prediction is a technique that calculates a predictive signal value based on previous signal values. The difference between the predicted value and the actual value is called prediction error, which is used in entropy coding instead of the absolute values. There are linear and non-linear predictors: The linearity results from the mathematical formula for calculating the prediction.

The discrete cosine transform (DCT) and the DWT presented in Section 2.2.2.1 are often used for spatial decorrelation of image data. A general one-dimensional discrete signal transform can be expressed by

$$
X[k]=\sum_{n=0}^{N-1} x[n] \cdot a[k, n], \quad k=0,1, \ldots, N-1
$$

where $x[n]$ denotes a discrete signal with length $N$, and $a[k, n]$ denotes a forward transform kernel. The inverse transform is as follows:

$$
x[n]=\sum_{k=0}^{N-1} X[k] \cdot b[n, k], \quad n=0,1, \ldots, N-1
$$

where $b[n, k]$ is an inverse transform kernel. The signals $x[n]$ and $X[k]$ can be interpreted as column vectors: The signal $\underline{x}$ can be transformed into $\underline{X}$ using a transform kernel $\mathbf{A}$ :

$$
\underline{X}=\mathbf{A} \cdot \underline{x}
$$

The transform is reversible if $\mathbf{A}^{-1}$ exists. The inverse transform is defined as follows: $\mathbf{B}=\mathbf{A}^{-1}$ :

$$
\underline{x}=\mathbf{B} \cdot \underline{X}, \quad \mathbf{B}=\mathbf{A}^{-1}
$$




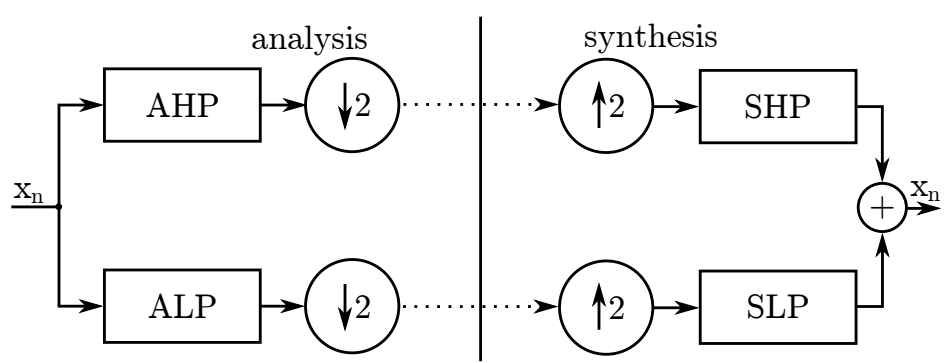

Figure 2.13: Two-channel filter bank for signal analysis and synthesis.

Most transforms are separable, i. e. a two-dimensional transform can be achieved by applying the one-dimensional transform to rows and columns. The transform coefficients $\underline{X}$ are the weights of each basis function. A well-chosen transform kernel allows describing the signal with fewer significant basis functions, which results in a higher decorrelation (or compression) of the signal.

A filter bank decomposes a signal into frequency components. A filter bank has a structure for decomposition (analysis) and reconstruction (synthesis). Figure 2.13 shows a two-channel filter bank for signal analysis and synthesis. The analysis high-pass filter (AHP) and the analysis low-pass filter (ALP) are used for signal analysis, whereas the synthesis high-pass filter (SHP) and the synthesis low-pass filter (SLP) are used for signal synthesis.

\subsubsection{Coding}

Coding can be divided into two groups: pre-coding and entropy coding [107]. Both are lossless data compression schemes that take advantage of the distribution of uncorrelated data or symbols. In pre-coding, symbols are mapped to symbols of a different alphabet, whereas in entropy coding, short codewords are assigned to symbols with a high probability of occurrence and longer codewords are assigned to symbols with a low probability of occurrence.

The goal of pre-coding is to minimize the inter-symbols redundancy, e. g. run-length coding. The goal of entropy coding is to bring the memory requirements closer to the signal's entropy. This can be either done by substitution of symbols with bit sequences (codeword-based), or by substitution of symbol sequences with a bit-stream (arithmetic) [107]. Known representatives of codeword-based entropy coding schemes are ShannonFano coding, Huffman coding, and Colomb-Rice coding [107]. Arithmetic coding is, for example, used in context-adaptive binary arithmetic coding (CABAC) which is the entropy encoder in JPEG2000 [54].

In 1952, David A. Huffman developed a variable-length code and the most efficient compression method for substitution of symbols with bit sequences [50]. Basically Huffman has developed a method for constructing an optimal code using a tree structure. 
The method is presented in detail in $[107,123]$. Huffman coding is used in JPEG and can be enabled in JPEG2000 [53, 54].

\subsubsection{Wavelet Transform}

The term wavelet is derived from the French word "ondelette" and means something like "small wave". It refers to the wavelet theory underlying basis functions - the socalled wavelets. These are - in contrast to the basis functions of classical orthogonal transform like Fourier or cosine transform - finite energy signals ${ }^{11}$. As with a short-time Fourier transform with windowing, a simultaneous time and frequency analysis is possible.

The wavelet transform uses a variable window size: High-frequency components of a signal are represented with a high time resolution, whereas low-frequency components are represented with a low time resolution. The basis function $\psi$ of a wavelet transform is called mother-wavelet (or generating function). All wavelets are generated by scaling (parameter $p$ ) or shifting (parameter $q$ ):

$$
\psi_{p, q}(t)=\frac{1}{\sqrt{p}} \cdot \psi\left(\frac{t-q}{p}\right) \quad p, q \in \mathbb{R}, p>0
$$

The Fourier transform $\Psi_{p, q}(\omega)$ of the basis function $\psi_{p, q}(t)$ is as follows:

$$
\Psi_{p, q}(\omega)=\int_{-\infty}^{\infty} \frac{1}{\sqrt{p}} \cdot \psi^{*}\left(\frac{t-q}{p}\right) \mathrm{e}^{-i \omega t} \mathrm{~d} t
$$

where $\psi^{*}(t)$ is the complex conjugate of the mother wavelet $\psi(t) . \Psi_{p, q}$ is used in the admissibility condition, which is a sufficient condition for the existence of the wavelet:

$$
C_{\psi}=\int_{0}^{\infty} \frac{|\Psi(\omega)|^{2}}{|\omega|} \mathrm{d} \omega<\infty
$$

The admissibility condition also implies that $\Psi_{p, q}(\omega=0)=0$ and $\int_{-\infty}^{\infty} \psi(t) \mathrm{d} t=0$. The continuous wavelet transform ${ }^{12}$ of a signal $f(t)$ and the wavelet function $\psi_{p, q}$ with scaling parameter $p$ and shifting $q$ :

$$
W T(p, q)=\int_{-\infty}^{\infty} f(t) \psi_{p, q}(t) \mathrm{d} t=\frac{1}{\sqrt{p}} \int_{-\infty}^{\infty} f(t) \psi^{*}\left(\frac{t-q}{p}\right) \mathrm{d} t
$$

\footnotetext{
${ }^{11}$ The energy $E_{s}$ of a continuous-time signal $x(t)$ is defined as $E_{s}=\int_{-\infty}^{\infty}|x(t)|^{2} d t$. A signal has finite energy, if $E_{s}<\infty$.

${ }^{12}$ The forward wavelet transform is also called wavelet analysis.
} 
Haar wavelet $p=0, q=1$

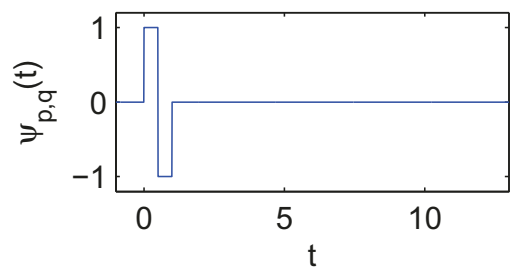

Haar wavelet $p=0, q=2$

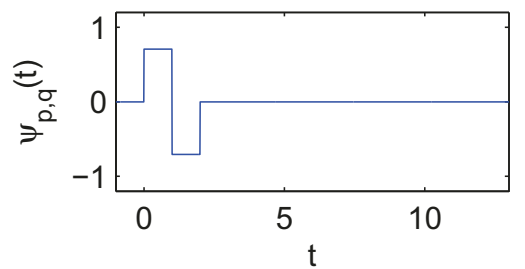

Haar wavelet $p=0, q=4$

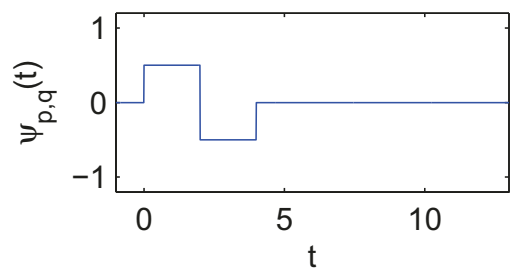

Haar wavelet $p=1, q=1$

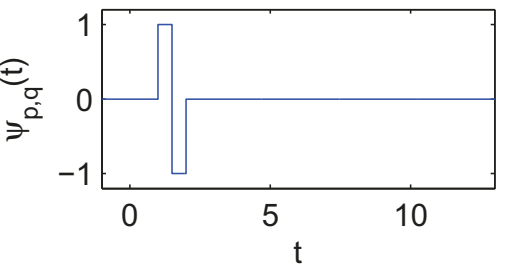

Haar wavelet $p=2, q=2$

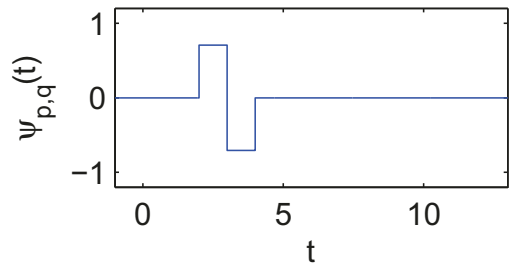

Haar wavelet $p=4, q=4$

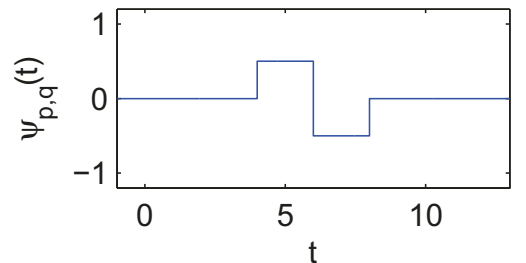

Haar wavelet $p=2, q=1$

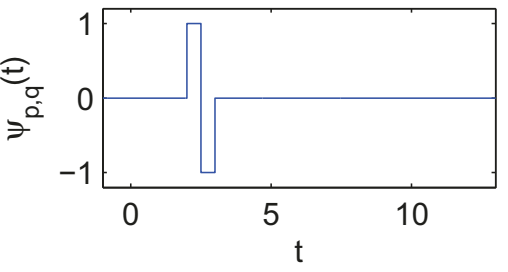

Haar wavelet $p=4, q=2$

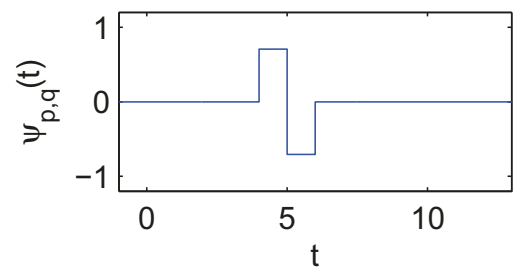

Haar wavelet $p=8, q=4$

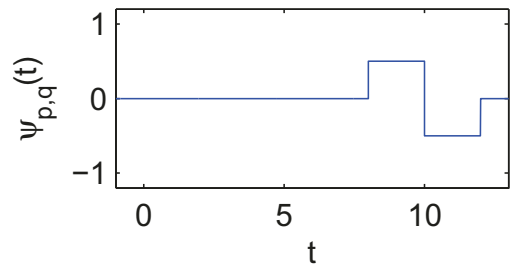

Figure 2.14: Some basis functions of the Haar wavelet. $p$ shifts the wavelet along the $t$-axis whereas the wavelet is scaled by $q$.

The constant $C_{\psi}$ derived with the admissibility condition can also be used for the inverse continuous wavelet transform ${ }^{13}[79]$ :

$$
f(t)=\frac{1}{C_{\psi}} \int_{0}^{\infty} \int_{-\infty}^{\infty} W T(p, q) \frac{1}{p^{2}} \psi_{p, q}(t) \mathrm{d} q \mathrm{~d} p
$$

\subsubsection{Discrete Wavelet Transform}

The DWT is used when a signal is in discrete form. The parameters $p$ and $q$ can be discretized as follows [107]:

$$
p=2^{-j} \quad q=k \cdot q_{0} \cdot 2^{-j} \quad j, k \in \mathbb{N}
$$

where $j$ denotes the resolution level, $k$ denotes the shift parameter, and $q_{0}$ denotes the length of the signal to be transformed. The basis functions used for the DWT can be derived from Equation 2.22:

$$
\psi_{j, k}\left(t_{n}\right)=\frac{1}{\sqrt{2^{j-1}}} \cdot \psi\left[\frac{n-k \cdot q_{0} \cdot 2^{-j}}{2^{-j}}\right]=\sqrt{2^{j}} \cdot \psi\left[n \cdot 2^{j}-k \cdot q_{0}\right]
$$

\footnotetext{
${ }^{13}$ The inverse wavelet transform is also called wavelet synthesis.
} 


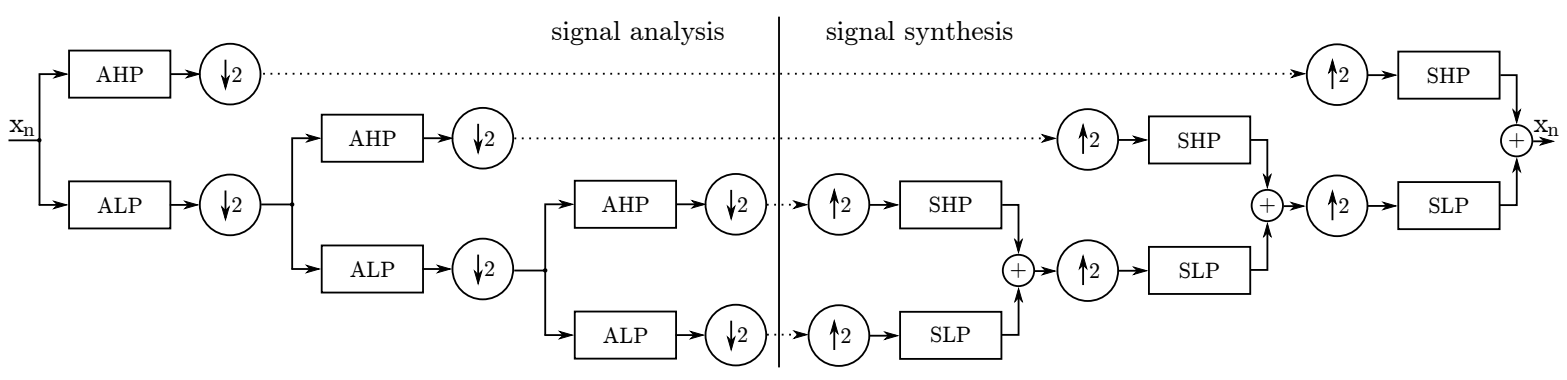

Figure 2.15: Filter bank for signal analysis and synthesis (3 stages).

The DWT equations for signal analysis and synthesis for a discrete signal of length $N$ are as follows:

$$
c_{j, k}=\sum_{n=0}^{N-1} f\left(t_{n}\right) \psi_{p, q}\left(t_{n}\right) \quad \text { and } \quad f\left(t_{n}\right)=\sum_{j} \sum_{k} c_{j, k} \psi_{j, k}\left(t_{n}\right) .
$$

Almost all practically useful DWTs use discrete-time filter banks. Most often, the DWT is performed with non-recursive finite impulse response (FIR) filters.

\subsubsection{Wavelet Filter}

In practice, the DWT is typically performed using a pair of FIR filters: The signal $x$ is first supplied to a low-pass and a high-pass filter and implicitly sub-sampled at a factor of two. The result of the high-pass filtering is the detail whereas the low-pass filtering produces the approximation of the signal. If this process is continuously applied, it is called dyadic decomposition. Figure 2.15 shows the process of signal analysis and synthesis using low-pass and high-pass filters for three decomposition levels. The separation can generally be reversed so that the signal $x$ is perfectly reconstructed.

A signal vector $x$ can be decomposed into the low-pass coefficients

$$
s(n)=\sum_{i=0}^{P-1} h(i) \cdot x(2 n-i)
$$

and the high-pass coefficients

$$
d(n)=\sum_{i=0}^{P-1} g(i) \cdot x(2 n-i)
$$

where $h$ and $g$ are the low-pass and high-pass filters, respectively. The number of filter stages (and coefficients) depends on the wavelet. The filter design is described in detail in [107]. Table 2.1 shows the analysis and synthesis coefficients for commonly used wavelet filters. The Haar wavelet was proposed by the Hungarian mathematician Alfréd Haar in 
1909 and is the first known wavelet. Furthermore, it is the simplest wavelet and is given by two rectangular functions:

$$
\psi(t)_{\mathrm{HAAR}}= \begin{cases}1 & 0 \leq t<0.5 \\ -1 & 0.5 \leq t<1 \\ 0 & \text { otherwise }\end{cases}
$$

The Cohen-Daubechies-Feauveau (CDF) $9 / 7$ and 5/3 wavelets presented in [23] are often used for image compression. The values are derived from the length of the high- and low-pass filters.

Table 2.1: Filter coefficients of commonly used wavelet filters.

\begin{tabular}{lll}
\hline & high-pass & low-pass \\
\hline Haar & $h_{0,0}=\frac{1}{2}$ & $h_{1,0}=\frac{1}{2}$ \\
& $h_{0,1}=-\frac{1}{2}$ & $h_{1,1}=\frac{1}{2}$ \\
\hline $\operatorname{CDF}(5 / 3)$ & $h_{0,1}=h_{0,3}-\frac{1}{4}$ & $h_{1,0}=h_{1,4}=-\frac{1}{8}$ \\
& $h_{0,2}=\frac{1}{2}$ & $h_{1,1}=h_{1,3}=\frac{1}{4}$ \\
& & $h_{1,2}=\frac{3}{4}$ \\
\hline $\operatorname{CDF}(9 / 7)$ & $h_{0,0}=h_{0,8} \approx 0.0267$ & $h_{1,1}=h_{1,7} \approx 0.0913$ \\
& $h_{0,1}=h_{0,7} \approx-0.0169$ & $h_{1,2}=h_{1,6} \approx-0.0575$ \\
& $h_{0,2}=h_{0,6} \approx-0.0782$ & $h_{1,3}=h_{1,5} \approx-0.5913$ \\
& $h_{0,3}=h_{0,5} \approx 0.2669$ & $h_{1,4} \approx 1.1151$ \\
& $h_{0,4} \approx 0.6029$ & \\
\hline
\end{tabular}

\subsubsection{The Lifting Scheme}

Lifting is a method to simplify the arithmetic structure of a wavelet filter. It splits the filter into one or more predict $(\mathrm{P})$ and update $(\mathrm{U})$ steps, thus reducing the number of operations [109].

Independently from the wavelet, the input signal $x=x_{0}, x_{1}, \ldots, x_{N-1}$ with length $N$ is split (S) into two signals $x_{2 n}$ and $x_{2 n+1}$ for $n=0, \ldots, \frac{N-1}{2}$. Depending on the wavelet, one or more lifting stages are necessary. In the case of a single-stage lifting, a prediction value is calculated from $x_{2 n}$ and subtracted from $x_{2 n+1}$ :

$$
d_{n}=x_{2 n+1}-P\left(x_{2 n}\right) .
$$

The result of this operation corresponds to the detail signal $d_{n}$. In the update step (U), $d_{n}$ is used to calculate the approximation signal $s_{n}$ :

$$
s_{n}=x_{2 n}+U\left(d_{n}\right) .
$$


The advantages of this approach are, that it can be implemented in-place, i. e. no additional memory is needed, and, in general, that it needs less operations for the transformation. The inverse transform can be obtained by a simple inversion of the equations:

$$
f_{2 n+1}=d_{n}+P\left(x_{2 n}\right) f_{2 n} \quad=s_{n}-U\left(d_{n}\right)
$$

With the lifting approach, the $\mathrm{CDF}(5 / 3)$ wavelet

$$
\begin{aligned}
& d_{n}=-\frac{1}{4} f_{2 n}+\frac{1}{2} f_{2 n+1}-\frac{1}{4} f_{2 n+2} \\
& s_{n}=-\frac{1}{8} f_{2 n-2}+\frac{1}{4} f_{2 n-1}+\frac{3}{4} f_{2 n}+\frac{1}{4} f_{2 n+1}-\frac{1}{8} f_{2 n+2}
\end{aligned}
$$

can be rewritten as follows:

$$
\begin{aligned}
d_{n} & =f_{2 n+1}-\frac{1}{2}\left(f_{2 n}+f_{2 n+2}\right) \\
s_{n} & =f_{2 n}+\frac{1}{4}\left(d_{n-1}+d_{n}\right) .
\end{aligned}
$$

Note that these formulas are identical except a scaling of the high-pass filter with a factor of $\frac{1}{2}$. The $\operatorname{CDF}(9 / 7)$ wavelet has the lifting structure shown in Figure 2.16. The lifting coefficients are as follows:

$$
\begin{aligned}
& \alpha \approx-1.5861 \\
& \beta \approx-0.0530 \\
& \gamma \approx 0.8829 \\
& \delta \approx 0.4435 \\
& k \approx 1.1496
\end{aligned}
$$

\subsubsection{Integer Wavelet Transform}

Albeit the DWT can be arithmetically reversible, its realization on computers leads to deviations caused by the limited precision of the floating point representation of numbers. Compression methods usually operate on fixed-point arithmetic because of its lower complexity and faster execution time. Rounding to the nearest fixed-point integer value causes further loss of information. An approach to overcome these issues is presented in [20, 109].

A major issue of this approach is, that a quantization of the derived wavelet coefficients causes artifacts after the inverse transform [11]. This method is therefore suitable only for lossless compression, and should not be used in conjunction with quantization or reduction. An integer version of the $\operatorname{CDF}(9 / 7)$ wavelet for lossless compression is presented in Section 2.4.2. 


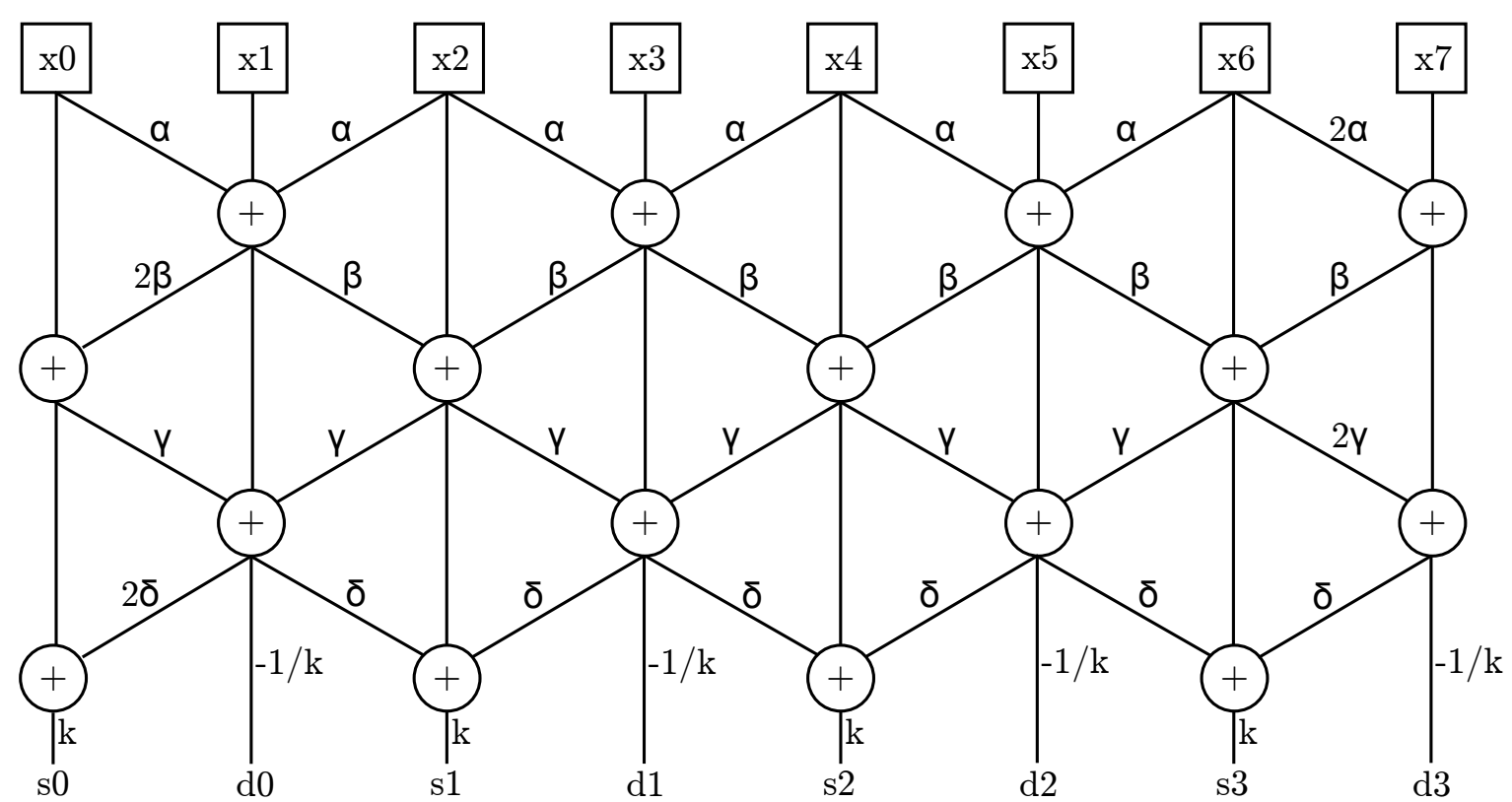

Figure 2.16: Structure of the analysis filter of the $\operatorname{CDF}(9 / 7)$ wavelet.

\subsubsection{Wavelet Transform of Images}

Usually, the DWT of images can be separated into two one-dimensional transforms. Here, the row and column transform are performed sequentially in any order. Depending on the wavelet filter, the image has to be extended at the borders. This can be achieved

- by filling with constant values (e.g. zero padding),

- by a periodic extension or

- by mirroring at the borders.

The first two approaches will generally lead to high-frequency interference, because of artificial edges introduced by the image extension. A following image compression algorithm will give worse results in most cases. Wavelet-based compressions algorithms are based, among other things, on the assumption, that high-frequency coefficients are usually smaller than the corresponding low-pass coefficients.

Figure 2.17 shows the transform steps of a separable two-dimensional DWT. The result of the transform is independent of the order of row and column transform. The four sub-bands resulting from the two-dimensional DWT have half the resolution of the original image ( $1 / 4$ of the pixels):

$L L$ contains the low-pass coefficients of both row and column transform and shows an approximation of the image.

$L H$ contains the coefficients from horizontal low-pass and vertical high-pass filtering and shows vertical edges. 


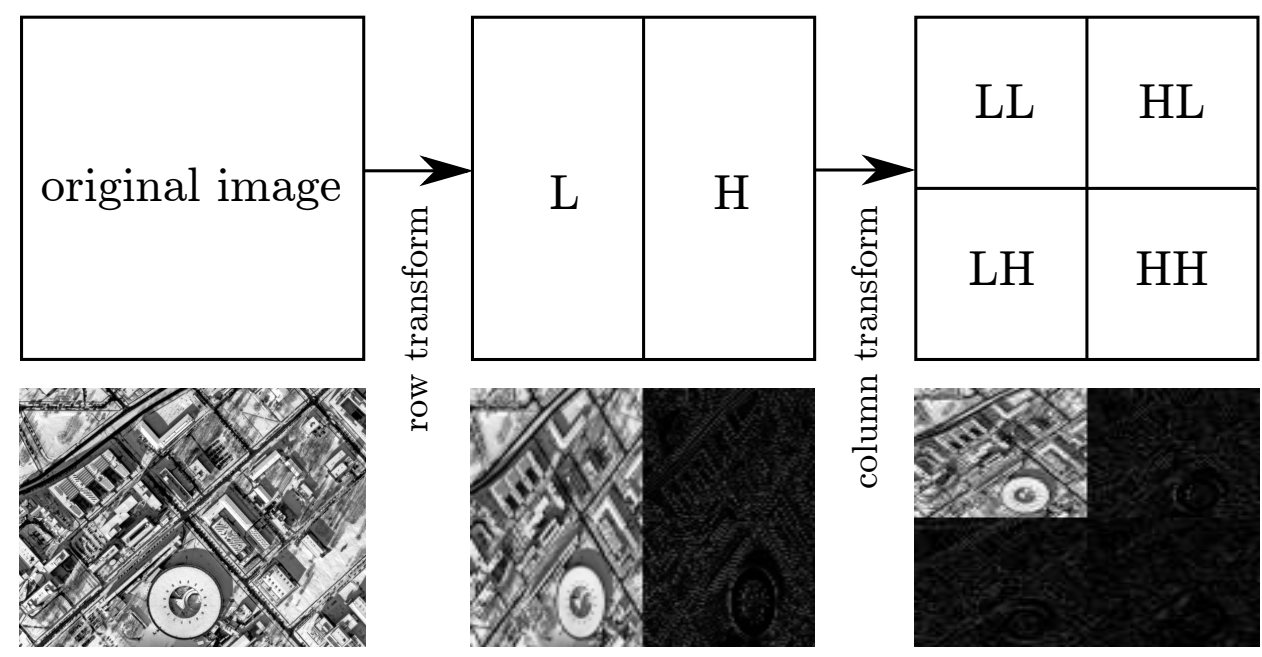

Figure 2.17: Row and column transform of a two-dimensional DWT.

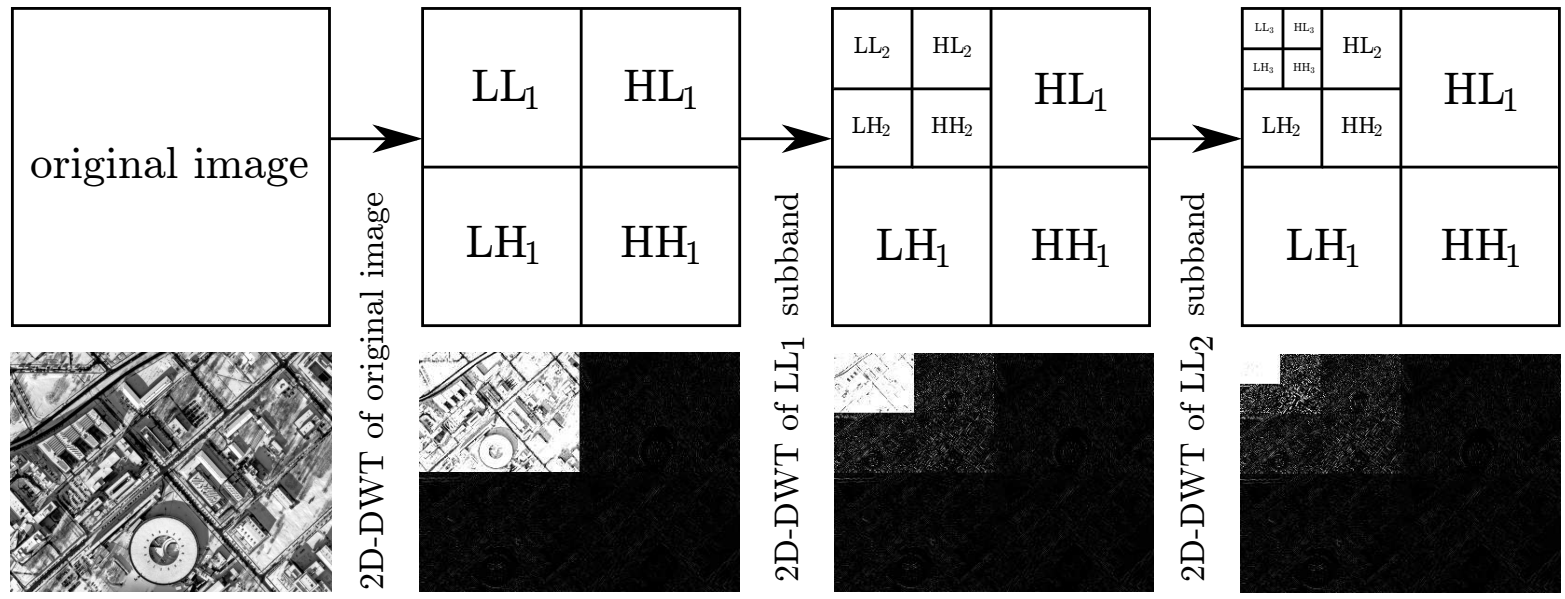

Figure 2.18: Three-stage two-dimensional DWT.

$H L$ contains the coefficients from horizontal high-pass and vertical low-pass filtering and shows horizontal edges.

$H H$ contains the high-pass coefficients of both row and column transform and show diagonal edges.

In wavelet-based compression algorithms, a three-stage dyadic decomposition is often used. Figure 2.18 shows the principle of this decomposition. The original image is decomposed into ten sub-bands: $L L_{3}, H L_{\{3,2,1\}}, L H_{\{3,2,1\}}$, and $H H_{\{3,2,1\}}$. The hierarchy of the wavelet-coefficients can be represented in a quad-tree. A quad-tree is a tree structure in which each internal node has four children. In the example of the three-stage decomposition, the coefficients $\mathrm{HL}_{3}, \mathrm{LH}_{3}$ and $\mathrm{HH}_{3}$ become the root of a quad-tree. The four coefficients of the corresponding lower sub-band become the children of each root and the so on. 


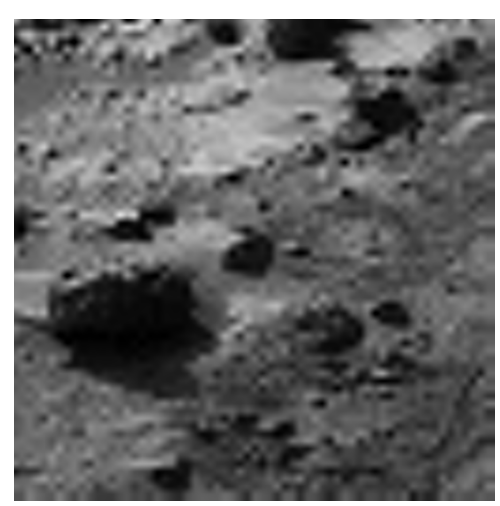

(a) $0.1 \mathrm{bpp}$

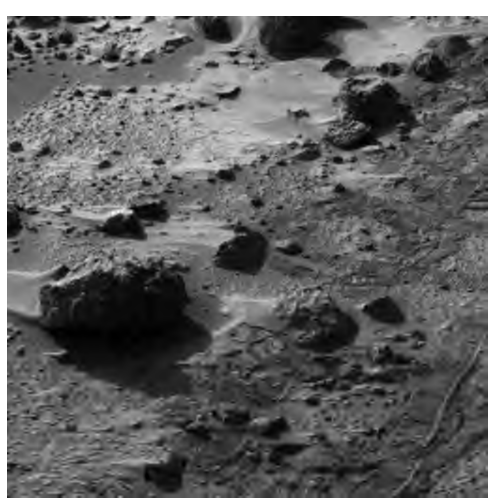

(b) $0.3 \mathrm{bpp}$

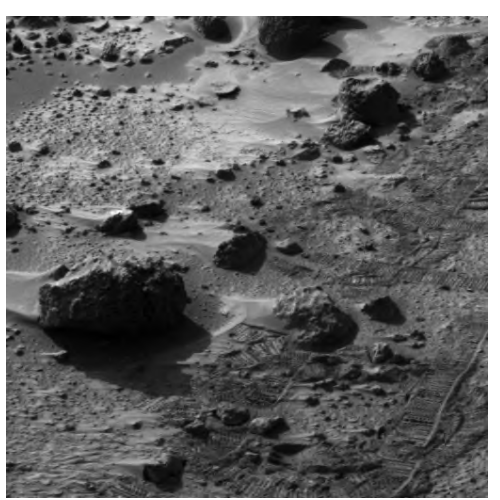

(c) $1.0 \mathrm{bpp}$

Figure 2.19: Image marstest compressed at different compression ratios.

\subsubsection{Wavelet-Based Image Compression Algorithms}

In recent years, the DWT is increasingly being used for spatial decorrelation of image data before entropy coding. The reason is that DWT-based image compression algorithms achieve the lowest distortion and the highest visual quality yet reported. There is a broad theoretical basis for satellite image data compression. As shown in [134], methods like $\mathrm{BTC}^{14}, \mathrm{DPCM}^{15}$, rice coding, DCT, DWT, and compression algorithms like JPEG, JPEG-LS, JPEG2000, ICER, or CCSDS 122.0-B-1 have been used in satellite imagery. Table 2.2 shows recent space missions and the used compression algorithm.

All DWT-based algorithms take advantage of the hierarchy of wavelet coefficients for efficient coding (see Section 2.2.2.5): First, an information compression takes place in the approximation of the signal (the wavelet coefficients of the low-pass filter are on average larger than that of the high-pass filter; the signal's energy is concentrated in the low-pass coefficients). Second, the low-pass coefficients are more important for visual quality. DWT-based compression algorithms usually work progressively, i.e. the visual quality of the decompressed image increases with the number of bits used for compression. This is also called embedded coding. Figure 2.19 shows this concept for the image marstest. Thus, all DWT-based compression algorithms support lossy compression, whereas lossless compression is only supported when the DWT is reversible and no quantization is used [18-20]. A target bit rate is achieved by termination of the coding process or by truncating the (already encoded) bit-stream.

Note that video data compression techniques such as motion compensation are not applicable for image data compression. It can be assumed, that the inter-frame correlation of satellite image data acquired by a push-broom sensor is nearby zero. Thus, algorithms like H.264 or High Efficiency Video Coding (HEVC) are not treated in this thesis.

\footnotetext{
${ }^{14}$ block truncation coding (BTC)

${ }^{15}$ differential pulse code modulation (DPCM)
} 
An wide overview on wavelet-based image compression algorithms is given in [108]. Starting with the method EZW ${ }^{16}$ presented in [102], a number of DWT-based methods for image data compression were developed in the academic research: SPIHT $^{17}$, $\mathrm{SPECK}^{18}, \mathrm{WDR}^{19}, \mathrm{ASWDR}^{20}$, and ICER, just to name a few $[52,60,85,94,113,116$, 117]. Furthermore, standards for wavelet-based image compression have been developed: JPEG2000 and CCSDS 122.0-B-1 [22, 24, 25]. To achieve optimal compression results on multi-spectral images, inter-band decorrelation or spectral compression techniques can be used. This is obvious because image data usually has correlation between spectral bands. There is a development to extend recent methods to be able to compress multi-spectral images, e.g. ICER-3D, 3D-SPIHT, 3D-SPECK [61, 74, 105].

EZW was originally designed to encode images but it can also encode multi-dimensional data [116]. As the name suggests, it is an embedded encoder which uses a DWT for spatial decorrelation. The "Z" in the name stands for Zero-tree which is the entropy coding method used: EZW encodes the wavelet coefficients in decreasing order and in multiple passes. In each pass, the magnitudes of the coefficients are compared with a threshold and, if necessary, encoded. The coding operates on quad-trees. A zero-tree is a quad-tree whose value in the root is less than or equal to its values in its leaves. EZW uses powers of two as threshold, which is often also referred to as bit plane coding. A simple example for coding with EZW is explained in [116].

The SPIHT algorithm was presented in [94]. SPIHT divides the transformed image into Spatial Orientation Trees, which are identical to quad-trees. Like all wavelet-based methods, it exploits the properties of the discrete wavelet transform for efficient coding. However, compared to EZW an even better compression ratio can be achieved.

JPEG2000 was released in December 2000 and supports both lossy and lossless compression of images [54]. It supports ROI coding in order to support different resolutions in a single image. The entropy coding stage is called Embedded Block Coding with Optimized Truncation (EBCOT).

$\mathrm{ICER}^{21}$ is the first wavelet-based compression algorithm designed for deep-space applications [60]. ICER-3D is an extension of the ICER algorithm for hyper-spectral image data $[61]$.

In 2005, the CCSDS-122.0-B-1 standard developed especially for space applications was released. It is the basis algorithm of the architecture presented in this thesis and will be presented in Section 2.4.

\footnotetext{
${ }^{16}$ Embedded Zerotree of Wavelet transforms (EZW)

${ }^{17}$ Set Partitioning In Hierarchical Trees (SPIHT)

${ }^{18}$ set partitioned embedded block coder (SPECK)

${ }^{19}$ Wavelet Difference Reduction (WDR)

${ }^{20}$ Adaptively Scanned Wavelet Difference Reduction (ASWDR)

${ }^{21} \mathrm{It}$ is actually not explained in the source, what this abbreviation means.
} 
Table 2.2: Compression algorithms employed for space missions.

\begin{tabular}{cr}
\hline Compression algorithm & Mission \\
\hline DCT-based & PHOBOS, CLEMENTINE, SPOT-5, CASSINI PROBE \\
\hline DPCM-based & SPOT-1, IKONOS, QuickBird, WorldView-1 \\
\hline JPEG-baseline & $\begin{array}{c}\text { TRACE, Proba-2, MicroLabSat, SUNSAT, TEAMSAT, PROBA-1 } \\
\text { Beijing-1, ALOS, Solar-B, Meteisat-8, Cartosat-1, Cartosat-2 }\end{array}$ \\
\hline JPEG-LS & FedSAT \\
\hline JPEG2000 & BilSAT-1, RASAT, IMS-1, X-SAT \\
\hline CCSDS 121.0-B-2 & Mars Odyssey, MTI, EO-1, PICARD, KOMPSat-3 \\
\hline CCSDS 122.0-B-1 & Proba-V, EnMAP, KOMPSat-3A \\
\hline ICER & Mars Exploration Rovers \\
\hline
\end{tabular}

\subsubsection{Image Quality Assessment}

Lossy and even near-lossless image compression introduces errors between the original raw image and the decompressed image. Depending on the compression ratio this might lead to visible distortions and artifacts. Lossy compression can often not be used in scientific missions because even small derivations in the acquired image data might lead to a misinterpretation of the data. Nevertheless, it can be used for applications, where derivations in the acquired image data can be tolerated.

As already mentioned in Section 2.1.1, the term image quality is used to describe the image degradation compared to an ideal or reference image. Image quality assessment (IQA) can be used to characterize the impact of image data compression on image quality. In the research area of image compression, statistical distortion metrics are often used. However, in remote sensing, statistical distortion metrics are often not suitable to determine image quality degradation because the main intention of image acquisition is often to process images automatically by algorithms. Furthermore, from the engineering point of view, determining the final image quality of the system or designing a system for a given image quality is a desired goal. A system theoretical approach to determine the impact of image data compression on the MTF is desirable or needed.

\subsubsection{Statistical Methods}

In principle, statistical methods can be divided into subjective and objective methods. In subjective IQA methods, a group of people are asked to give their opinion about the quality of each image. In order to provide reliable results, several standards like ITU-R BT.500-11 or ITU-R BT.1129-2 have been proposed [82]. The goal of objective IQA is to "design mathematical models that are able to predict the quality of an image accurately and also mathematically" [82]. An ideal objective IQA method should be able to mimic 
the quality evaluations of an average human observer.

Based on the availability of a distortion-free reference image, objective IQA methods can be classified into full-reference, no-reference, and reduced-reference methods. Fullreference means that two images, a distortion-free and a distorted, are used for IQA. Reduced-reference methods compare features or properties of a distortion-free and a distorted image. A no-reference metric uses only the distorted image.

Full-reference metrics are most often used to evaluate the performance of an image compression algorithm. Rate-distortion curves are used to describe the dependency between bit-rate and the image quality of the reconstructed image in comparison with the original image. Most often, metrics like mean absolute error (MAE), mean square error (MSE) or peak signal-to-noise ratio (PSNR) are used. The definitions for these metrics are as follows:

$$
\begin{aligned}
\operatorname{MAE}(X, Y) & =\frac{1}{M N} \sum_{i=1}^{M} \sum_{j=1}^{N}|X(i, j)-Y(i, j)| \\
\operatorname{MSE}(X, Y) & =\frac{1}{M N} \sum_{i=1}^{M} \sum_{j=1}^{N}[X(i, j)-Y(i, j)]^{2} \\
\operatorname{PSNR}(X, Y) & =10 \cdot \log _{10}\left(\frac{\mathrm{L}^{2}}{\operatorname{MSE}(X, Y)}\right) \mathrm{dB}
\end{aligned}
$$

where $M$ and $N$ denote the width and the height of the images $X(i, j)$ and $Y(i, j)$, and $L$ is the dynamic range of the pixel values (for $n$ bit unsigned integer it is $2^{n}-1$ ). Although they are almost always used, MAE, MSE, and PSNR produce not an adequate description of the visual quality. The problem is that different distortions can produce the same MSE or PSNR although they have very different image quality [120]. Structural similarity (SSIM) is a full-reference IQA metric, which involves structural information in the images. SSIM is calculated in various windows of a typical size of $8 \times 8$ :

$$
\operatorname{SSIM}(x, y)=\frac{\left(2 \mu_{x} \mu_{y}+c_{1}\right)\left(2 \sigma_{x y}+c_{2}\right)}{\left(\mu_{x}^{2}+\mu_{y}^{2}+c_{1}\right)\left(\sigma_{x}^{2}+\sigma_{y}^{2}+c_{2}\right)},
$$

with $\mu_{x}$ and $\mu_{y}$ are the averages, $\sigma_{x}^{2}$ and $\sigma_{y}^{2}$ are the variances, and $\sigma_{x y}^{2}$ is the covariance of windows $x$ and $y$. Commonly used values for the constants are $c_{1}=0.01$ and $c_{2}=0.03$. Its value lies in the interval $[0,1]$. The mean structural similarity (MSSIM) of two images can be calculated as follows:

$$
\operatorname{MSSIM}(X, Y)=\frac{1}{M} \sum_{i=1}^{M} \operatorname{SSIM}\left(x_{i}, y_{i}\right),
$$

where $M$ is the number of windows, and $x_{i}$ and $y_{i}$ are the corresponding regions of the images. The SSIM uses luminance, contrast, and structure comparisons to get a better visual quality prediction. There are a lot of other IQA metrics which were compared 
against a mean opinion score (MOS): VIF, VSNR or PSNR-HVS, just to name a few [86]. All these metrics are full-reference IQA metrics as they compare an undistorted with a distorted version of the image. In this context it should be noted, that possible noise of the original image most often is not taken into account. When using lossy image compression, knowledge about noise can be exploited to improve compression ratio.

\subsubsection{Systems Theory Approach}

The system theory approach of the MTF introduced in Section 2.1.1.2 may be used under certain circumstances - to characterize the impact of image data compression on image quality. A very useful tool to describe the image quality degradation is the MTF, which relates input and output of a linear (imaging) system. Using the MTF has the advantage that the overall system MTF can be determined as the product of the individual component MTFs.

Image compression algorithms can be understood as a component of the overall imaging system [46]. However, they are typically neither linear, i. e. not all the output frequencies correspond with the same input frequencies due to the irreversible quantization, nor iso-planar, i. e. they are space-variant because they use some sort of blocking of image pixel or coefficients. In [46], lossy compression was assumed to be linear to solve that problem. Nevertheless, the MTF gained from compressed images must be carefully assessed, because image compression can introduce edges and artifacts.

\subsection{Hardware Architectures}

\subsubsection{FPGA Hardware Architectures for Discrete Wavelet Transform}

The DWT can be applied to signals with any number of dimensions. For image or video compression, the one-, two-, and three-dimensional DWT are suitable. There are a number of different FPGA architectures and storage requirements, depending on the number of dimensions and the used wavelet. The most commonly used FPGA architectures are presented in [21, 49]. The result of the DWT are not necessarily integer coefficients which would be desirable. An approach in order to construct an integer-to-integer DWT was proposed in $[18,19]$. Additionally, the lifting scheme is often used to design a wavelet filter and to efficiently perform the DWT [109] on an FPGA platform.

\subsubsection{FPGA-Based One-Dimensional DWT}

As already mentioned, the DWT can be realized by various architectures. The most obvious solution is to use a FIR filter, leading to a convolution-based architecture. A FIR filter can be optimized with methods such as parallelization, serialization, and pipelining. A parallel FIR filter puts the input values into a shift register, performs a multiplication 

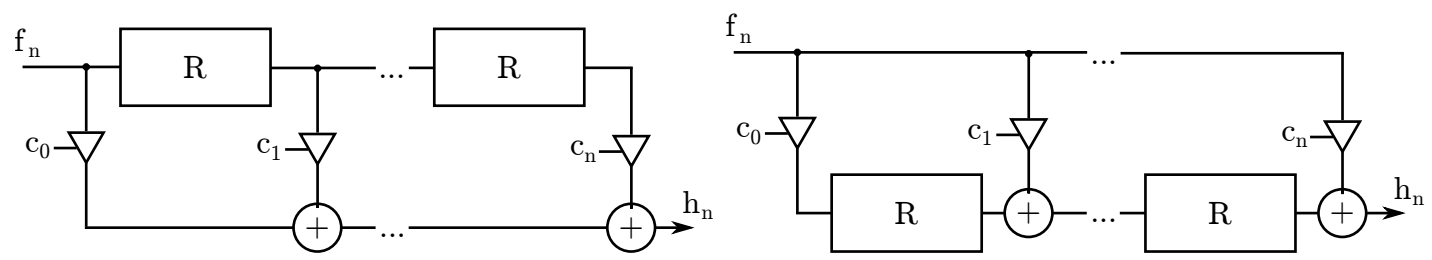

Figure 2.20: Parallel and quasi serial FIR filter.

for every filter stage and summarizes the results. The timing path of a single operation is as follows:

$$
\Delta T=T_{m}+n \cdot T_{a}
$$

where $T_{m}$ denotes the duration of the (parallel) multiplication, $T_{a}$ denotes the duration of the addition and $n$ denotes the depth of the adder tree. The registers can be placed behind the multipliers so that the timing path is shortened to the length $T_{m}+T_{a}$ (see Figure 2.20).

The DWT can also be performed by a lifting-based architecture (see Section 2.2.2.3). The advantages of this architecture are as follows [5]:

- Depending on the length of the FIR filter, the number of arithmetic operations can be reduced by half.

- By using in-place operations, no additional memory is required.

- The handling of boundary values is simplified by the structure of the modified filter.

A direct mapping to registers, multipliers, and adders can exploit the advantages of the lifting scheme. For an efficient FPGA implementation, however, the critical time path must be optimized. The flipping architecture is a special case of the direct-mapped architecture, wherein the timing path is optimized by the replacement of some multiplications [21].

There are other architectural features for certain wavelets: The high-pass and lowpass coefficients for the Haar wavelet can be calculated directly from two consecutive input values, whereas the calculation for other wavelets is more complex: The analysis coefficients of the often used $\operatorname{CDF}(9 / 7)$ wavelet can be determined with the structure shown in Figure 2.16. Because of structural similarity, a folded architecture shares multipliers and reduces the resource consumption. The disadvantage of the folded architecture is the increased complexity of the control unit. It is of course also possible to perform the calculations in a pipeline, whereby the number of registers may increase dramatically. The filter coefficients of the $\mathrm{CDF}(5 / 3)$ wavelet are powers of two, so that the multiplications can be realized by bit-shift operations. 


\subsubsection{FPGA-Based Two-Dimensional DWT}

The wavelet transform of image lines requires little memory for adjacent pixels, while a larger memory is required for the wavelet transform of the image columns in order to buffer complete lines. It is assumed, that the scan order of the image is line by line from left to right and from top to bottom. If the wavelet transform is separable (see Section 2.2.2.5), the column transform can be performed after the row transform, or vice-versa (direct-scan architecture). The results are stored after each step in a memory and are read back for the next step. The memory size needed for this architecture corresponds with the image $\operatorname{size}^{22}$. For a $J$-level dyadic decomposition of the image, the number of memory transfers is as follows:

$$
\begin{aligned}
& 4 \cdot\left(1+\frac{1}{4}+\frac{1}{16}+\ldots+\left(\frac{1}{4}\right)^{J-1}\right) \cdot N \cdot M \quad \text { [words/image] } \\
& =4 \cdot \sum_{i=0}^{J-1} \frac{1}{4^{i}} \cdot N \cdot M \quad \text { [words/image] } \\
& \leq \frac{16}{3} \cdot N \cdot M \quad \text { [words/image]. }
\end{aligned}
$$

Since it is very inefficient to write the results after each transform step back into a memory, the so-called line-based architecture is often used in practice, which executes row and column transform interleaved and as early as possible: The image rows are first transformed and temporarily stored in a small buffer. The column transform starts, if the buffer is sufficiently filled. The number of rows that need to be stored in memory depends on the selected wavelet: The column transform using the Haar wavelet needs to store only one row, whereas the $\operatorname{CDF}(5 / 3)$ or $\operatorname{CDF}(9 / 7)$ wavelet requires to store two or six rows for the high-pass and four or eight rows for the low-pass, respectively. The lifting scheme reduces this numbers to three or five, respectively (see Section 2.2.2.3).

A multi-level DWT can obviously be performed by a cascade of multiple two-dimensional DWT module, but it is also possible to use only one module, since the number of pixels becomes smaller with each decomposition stage (at dyadic decomposition):

$$
\begin{aligned}
& \left(1+\frac{1}{4}+\frac{1}{16}+\ldots+\left(\frac{1}{4}\right)^{J-1}\right) \cdot N \cdot M \quad \text { [words/image] } \\
& =\sum_{i=0}^{J-1} \frac{1}{4^{i}} \cdot N \cdot M \quad \text { [words/image] } \\
& \leq \frac{4}{3} \cdot N \cdot M \quad \text { [words/image]. }
\end{aligned}
$$

If the $L L$ coefficients calculated by a two-dimensional wavelet core are sent to another core, the data rate will be only $25 \%$ of the original data rate. A folded architecture can be used for share resources: Assuming a utilization of $64 \%$ of the first wavelet

\footnotetext{
${ }^{22}$ neglecting the increase of the dynamic range of the coefficients
} 
core, a second core will have a utilization of $16 \%$ and a third of only $4 \%$. Neglecting the increased complexity of the controller, the total utilization of the wavelet core will be $84 \%$. A trade-off between the complexity of a single transform core and resource consumption of all cores must be found.

In [21], further architectures for the two-dimensional wavelet transform are presented.

\subsubsection{FPGA-Based Three-Dimensional DWT}

The DWT can also be performed along the time axis (time filtering) or along the spectral axis (spectral filtering). In image sequences or for video coding, time is usually regarded as the third dimension, so that one also speaks of a three-dimensional wavelet transform. Depending on the wavelet, one or more images must be stored in memory (see Section 2.3.1.2). In multi-spectral imagery, the DWT can be performed between the different spectral bands. If the image pixels of the different spectral channels arrive at the same time, the spectral DWT can be performed without any memory.

As for the one-dimensional and two-dimensional DWT, there are a number of different architectures for a three-dimensional DWT. These are basically the same as for the two-dimensional DWT.

\subsubsection{Hardware Architectures for On-Board Image Data Compression}

Basically, architectures for image data compression can be distinguished between online and offline approaches. Online indicates that the image data is compressed prior to storing, i. e. during acquisition. This requires, of course, an implementation that is fast enough to compress the images in real-time. In the case of an offline architecture, the image data are first stored and compressed after acquisition with a potential lower data rate. In both cases, the compression algorithm can be implemented in hardware, i. e. in a DSP, application-specific integrated circuit (ASIC) or FPGA, or in software running on a CPU. The latter are not considered in this thesis, since they are typically not suitable for high data rate systems.

The first known satellite with on-board image compression, was SPOT-1 (1980). It used DPCM with a fixed compression ratio (CR) of 1.3:1. The algorithm needed only three operations per pixel and was "the only type of algorithm compatible with the very limited capability of that time space qualified electronics" [66]. This compression scheme was also used for SPOT-2 to SPOT-4. A transform-based compression algorithm was first used for the PHOBOS (1988) mars exploration missions. The algorithm used a DCT for spatial decorrelation followed by scalar quantization and fixed length coding. Three different compression ratios were available: 4:1, 8:1 and 12:1. Compression was performed offline and in software on a Z80 [66, 134]. 
In subsequent years, the data rate of high-resolution systems has increased rapidly. In parallel, new image compression standards like JPEG have been developed. The JPEG standard was approved in 1992 and used in many remote sensing missions with moderate data rates. At that time, high-resolution systems such as IKONOS (1999), QuickBird (2001) or WorldView-1 (2007) are using relatively simple algorithms such as DPCM in order to perform image compression in real-time [16].

\subsubsection{ASIC-Based Compression Architectures}

In 1991, even before the JPEG standard was approved, the Centre national d'études spatiales (CNES) developed a JPEG-like compression ASIC capable of 4 Mpx/s real-time compression. Image Compression Module (ICM) was used in various space missions, e. g. CLEMENTINE (1994), CASSINI PROBE (1997) or ETS-7 (1997). For SPOT-5 (2002), a compression architecture targeted for Earth observation was developed [83, 112]. SPOT-5 contains three instruments producing up to seven data streams; each up to $128 \mathrm{Mbit} / \mathrm{s}$. The compression unit compresses up to three of the data stream with a fixed CR of 2.81:1.

A proprietary adaptive DPCM image compression algorithm was developed by Eastman Kodak Company. An ASIC called Bandwidth Compression Plus (BWCP) implementing the algorithm was developed and used in IKONOS (1999), QuickBird (2001), and WorldView-1 (2007). The ASIC achieved the operating rate of $22 \mathrm{Mpx} / \mathrm{s}$, supports lossless compression ratios of $2: 1$, and lossy compression ratios of $5: 1$ [16].

Airbus Defence and Space developed CoReCi ${ }^{23}$ which is used for SPOT-6 (2013), SPOT-7 (2014) or KazEOSat-2 (2014). As the name suggests, it is an image compression, mass storage, and ciphering unit. The system is available for a compression rate between $1.4 \mathrm{Gbit} / \mathrm{s}$ and $8.6 \mathrm{Gbit} / \mathrm{s}$, and a storage capacity between $1 \mathrm{Tbit}$ and $4 \mathrm{Tbit}$ [33]. The system uses multiple dedicated compression ASICs called WICOM ${ }^{24}$ with a speed of up to $25 \mathrm{Mpx} / \mathrm{s}$. Multi-Résolution par Codage de Plans Binaires (MRCPB), a wavelet-based image compression algorithm similar to JPEG2000 is used.

$\mathrm{CWICOM}^{25}$ was recently developed by Airbus Defence and Space in the context of an ESA contract [34]. It is an image compression ASIC which implements the CCSDS 122.0-B-1 standard. It supports both lossy and lossless image compression at a data rate of up to $60 \mathrm{Mpx} / \mathrm{s}$. It does not need external memory, since it contains almost $5 \mathrm{Mbit}$ of internal memory necessary for image compression. The ASIC bases on the Atmel ATC18RHA technology/cell library, which is intended to be used for space applications.

Table 2.3 gives a brief overview of the mentioned architectures.

\footnotetext{
${ }^{23}$ Compression Recording and Ciphering (CoReCi)

${ }^{24}$ Wavelet Image COmpression Module (WICOM)

${ }^{25}$ CCSDS Wavelet Image COmpression Module (CWICOM)
} 
Table 2.3: ASIC-based compressors for space applications.

\begin{tabular}{cccc}
\hline ASIC & Space mission & Compression algorithm & Compression throughput \\
\hline ICM & CLEMENTINE, ETS-7 & DCT-based & $4{ }^{\mathrm{Mpx} / \mathrm{s}}$ \\
\hline SPOT-5 compressor & SPOT-5 & DCT-based & $3 \times 128^{\mathrm{Mbit} / \mathrm{s}}$ \\
\hline BWCP & IKONOS, QuickBird, WorldView-1 & DPCM & $22^{\mathrm{Mpx} / \mathrm{s}}$ \\
\hline CoReCi/WICOM & SPOT-6, SPOT-7 & MRCPB & $1.4 \mathrm{Gbit} / \mathrm{s}$ to $8.6 \mathrm{Gbit} / \mathrm{s}$ \\
\hline CWICOM & - & CCSDS $122.0-\mathrm{B}-1$ & $60 \mathrm{Mpx} / \mathrm{s}$ \\
\hline
\end{tabular}

\subsubsection{COTS Hardware for On-Board Compression}

Components-off-the-shelf (COTS) can be used in order to reduce the development costs. With respect to the considered systems, COTS can either be related to software or hardware, i. e. a compression algorithm or a compression processor.

In 2000, CNES performed a study of JPEG2000 standard with radiation hardened components. Both CNES and Consultative Committee for Space Data Systems (CCSDS) concluded that JPEG2000 was too complex to be implemented on this kind of hardware [112]. The arithmetic coder and the rate allocation procedure were cited as a reason. The CCSDS then started to look for a new compression algorithm for the CCSDS-IDC recommendation. Section 2.4 presents the standard that has emerged from this study. In 2008, CNES started a study of the commercial JPEG2000 ASIC from Analog Devices [10]. Radiation tests were disastrous, since "not a single image was compressed successfully" [112].

Recent space missions have not employed general-purpose computation on graphics processing units (GPGPUs) for on-board compression due to the fact that radiation-tolerant or -hardened GPGPUs are not yet available. In principle, this should be feasible, but compared to recent non-space GPGPU technology, radiation immunization will effect an increase in die size and power consumption and a decrease in the maximum clock speed. However, many image compression standards which demand high throughput have been ported to GPGPU platform and future technology might allow space-qualified GPGPUs. Table 2.4 gives a brief overview of compression-related algorithm implementations using GPGPUs.

Another possibility is to synthesize COTS IP cores on a radiation hardened device such as the Xilinx Virtex-5QV [128]. In any case, radiation hardened memory as proposed in [27], may be used.

\subsubsection{FPGA-Based Compression Architectures}

In recent years, FPGAs have been increasingly used for space applications. The use of FPGAs has considerable advantages, since usually only prototypes are developed. As

\footnotetext{
${ }^{27}$ compared to the respective reference implementation
} 
Table 2.4: Compression-related algorithm implementations using GPGPUs [89].

\begin{tabular}{|c|c|c|c|c|}
\hline Related work & Algorithm & Speedup $^{27}$ & Reference CPU configuration & GPGPU card \\
\hline $\begin{array}{l}\text { GPU-Based DWT Acceleration for } \\
\text { JPEG2000, [78] }\end{array}$ & DWT & 68 & Intel Core i7, $3.2 \mathrm{GHz}, 6 \mathrm{~GB}$ RAM & NVIDIA GeForce GTX295 \\
\hline $\begin{array}{l}\text { A Parallel Implementation of the 2D } \\
\text { Wavelet Transform using CUDA, [37] }\end{array}$ & DWT & 20.8 & Intel Core 2 Quad Q6700, 2.66 GHz, 4 GB RAM & NVIDIA Tesla C870 \\
\hline $\begin{array}{l}\text { A GPU-Accelerated Wavelet } \\
\text { Decompression System with SPIHT, } \\
{[103]}\end{array}$ & IDWT & 158 & $2 \times$ Intel Xeon E5520, $2.27 \mathrm{GHz}, 16 \mathrm{~GB}$ RAM & NVIDIA Tesla C1060 \\
\hline $\begin{array}{l}\text { A novel parallel Tier-1 coder for } \\
\text { JPEG2000 using GPUs, [67] }\end{array}$ & JPEG2000 & $\begin{array}{c}100 \text { (DWT) } \\
30 \text { (Bit-Plane) } \\
17 \text { (Tier-1) }\end{array}$ & Intel Core i $7,2.8 \mathrm{GHz}, 12 \mathrm{~GB}$ RAM & NVIDIA Geforce GTX480 \\
\hline $\begin{array}{l}\text { A parallel implementation of } \\
\text { JPEG2000 encoder on multi-GPU } \\
\text { system, }[62]\end{array}$ & JPEG2000 & $\approx 100$ & $2 \times$ Intel Xeon W5590, $3.33 \mathrm{GHz}$ & $4 \times$ NVIDIA Geforce GTX 680 \\
\hline $\begin{array}{l}\text { GPU implementation of JPEG2000 for } \\
\text { hyperspectral image compression, [65] }\end{array}$ & JPEG2000 & - & Intel Core 2 Duo E8400, $3.00 \mathrm{GHz}, 6$ GB RAM & NVIDIA Geforce GTX480 \\
\hline
\end{tabular}

already mentioned in Chapter 1, the space environment delivers challenging requirements regarding reliability, power consumption, operating temperature range, vacuum, radiation, shock resistance or component qualification. Note that a temporary or permanent FPGA malfunction can lead to failure or loss of the mission, since a replacement or repair is usually not possible. A radiation effect can be propagated from a single semiconductor element through the system's hierarchy. There are a number of mitigation techniques [15, $59,106,127]$ in order to fulfill the requirements on reliability and radiation tolerance:

- In the simplest case, the system runs a periodic power cycle or uses a hardware or software watchdog, which enforces a power cycle in the case of an error.

- The FPGA is Radiation-Hardening-by-Design (RHBD).

- error detection and correction (EDAC) uses hamming or cyclic codes in order to detect and correct certain bit error. The number of bit errors which can be detected or corrected depends upon the implementation of EDAC.

- Dual redundancy duplicates critical parts of a system. An error can be detected, if the outputs of the systems differ from each other (hot redundancy). It is also possible to switch to a redundant module, if an error has been occurred (cold redundancy).

- triple modular redundancy (TMR) is a form of redundancy, in which critical components of a design are tripled and a majority-voting system is used to produce a single output. TMR might be supported by the FPGA itself [81, 128], applied by synthesis tools or can be performed manually.

- FPGA configuration read-back is often used to check, whether the configuration of the FPGA is still valid. A single event upset (SEU) might have changed the configuration of the FPGA. 
- Scrubbing periodically inspects and corrects errors using the FPGA configuration file. It reduces the likelihood that single correctable errors lead to an uncorrectable error.

- TMR and scrubbing can be performed either on the whole FPGA configuration or on parts of it (partial reconfiguration).

- FSM, counter hardening and some above techniques can also be used on netlist level.

GRACE (2002) and FedSAT (2002) were the first missions using an early Xilinx space grade FPGA. The design and implementation of the FedSAT image compression system is presented in [28]. The system is able to compress pixels at a rate of $43.8 \mathrm{Mpx} / \mathrm{s}$. In [90], a simplified implementation in VHSIC Hardware Description Language (VHDL) is described. The author uses a so called partitioned DWT, i. e. the image is partitioned into smaller blocks and the DWT is applied to each of these blocks. In principle, the block formation is done in the spatial domain instead of the wavelet domain. This results in blocking artifacts along the boundaries of each block. A variable fixed-point approach is presented in [38]. Instead of using floating-point arithmetic, it uses fixed-point arithmetic, where the decimal point's position is not locked at a specific position. Depending on the phase, an individual data throughput of $100 \mathrm{Mpx} / \mathrm{s}$ up to $224 \mathrm{Mpx} / \mathrm{s}$ was achieved. However, this approach is limited to an image size of $1024 \times 1024$ pixels and the throughput of the overall compression system, which is not necessarily the minimum of the individual throughput, was not measured.

An overview of FPGA-based image compression systems is presented in [134]. The authors also propose an architecture for on-board image compression.

The design and implementation of the FORMOSAT-5 (2015) Remote Sensing Instrument (RSI) is described in [71] and [72]. FORMOSAT-5 is an optical satellite with a GSD of $2 \mathrm{~m}$. The total output data rate of the instrument is $970 \mathrm{Mbit} / \mathrm{s}$ (PAN + four MS). Three space grade Xilinx XQR5VFX130 [128] are used for online image compression. One FPGA is used for PAN processing and two FPGAs are used for MS processing. The compression system uses 24 external SRAM chips, each 1 Mbyte.

An implementation of the CCSDS 122.0-B-1 standard used for Proba-V (2013) and EnMAP (2015) is presented in [70]. The authors use a Microsemi ProASIC3E for development and an anti-fuse RTAX2000S for flight model. The estimated data throughput is $173 \mathrm{Mbit} / \mathrm{s}$ at $66 \mathrm{MHz}$.

Table 2.3 gives a brief overview of the mentioned architectures. 
Table 2.5: FPGA-based compressors for space applications.

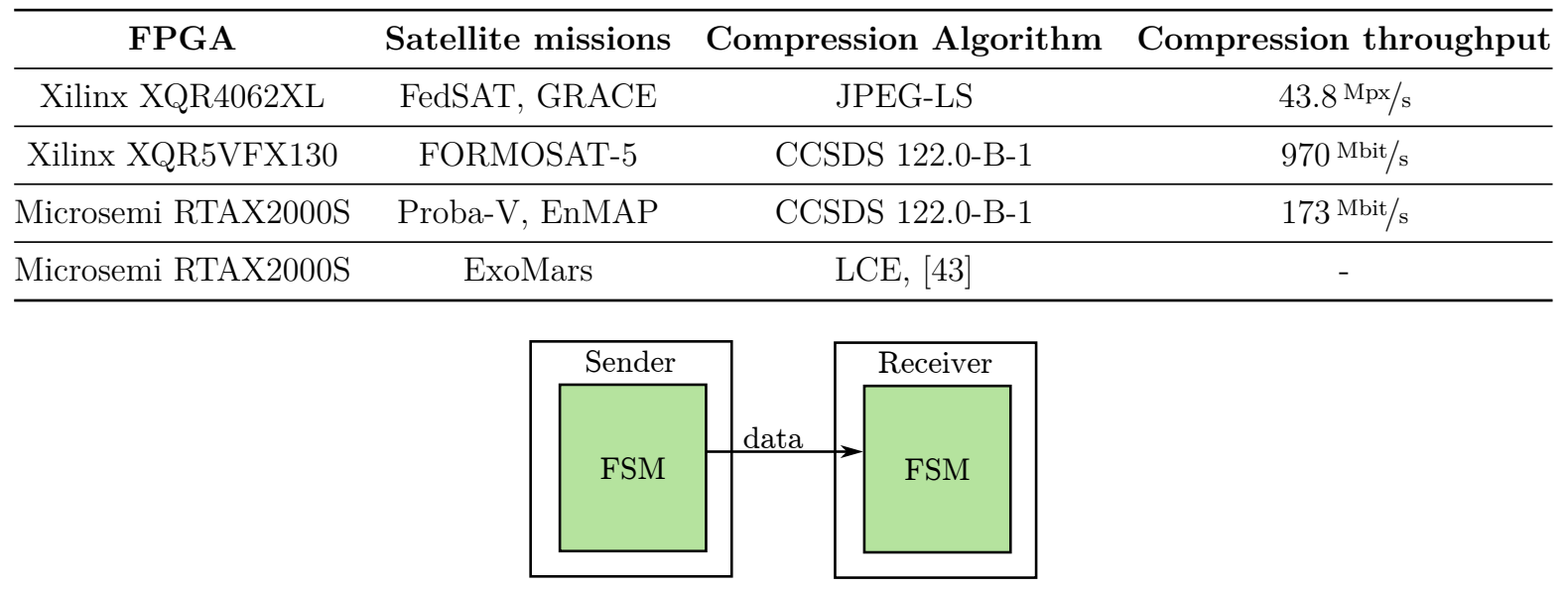

Figure 2.21: Structure of the Channel communication.

\subsubsection{Hardware Operating System}

An approach to increase re-useability and to reduce the engineering effort called Hardware Operating System was developped at the DLR and presented in [64]. It is a method for describing a hardware design independent of special hardware. It uses some basic principles of software operating systems, namely re-useability, information hiding and resource management. Since VHDL has fundamental limitations on the feasibility of these concepts, a pre-compiler was developed, which is also referred to as "structure compiler". The structure compiler links the application programs with the operation system modules and produces a VHDL program, which can be used for simulation or synthesis. Further advantages of this approach are that the development time is reduced and the reliability of the system can be increased.

The hardware operating system supports three communication interfaces: Channel, SChannel and Pipe. They are all used to transfer untyped data of any width. On the FPGA, a std_logic_vector is used for data transfer.

\subsubsection{Channel Communication Protocol}

The Channel interface implements an asynchronous transfer of a single data word. On the hardware side, this interface only consists of a std_logic_vector with a certain width. It can be used for read or write access to registers. Since it is an asynchronous interface, the sampling rate must be selected by the receiver. Figure 2.21 show the structure of the Channel communication.

\subsubsection{SChannel Communication Protocol}

The SChannel (Synchronous Channel) interface implements a synchronous single-word transfer. A FIFO has to be used for data flow control. If sender and receiver are in 


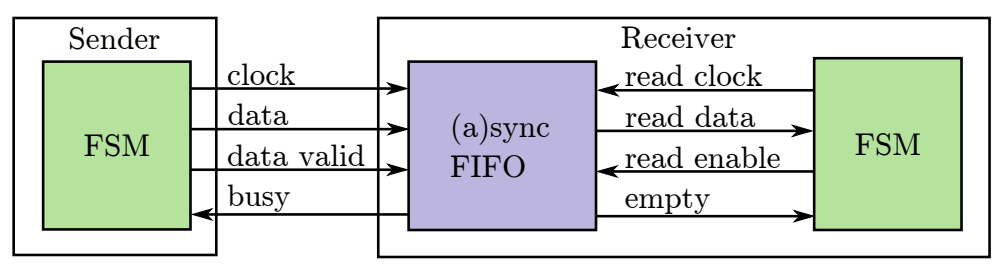

Figure 2.22: Structure of the SChannel communication.

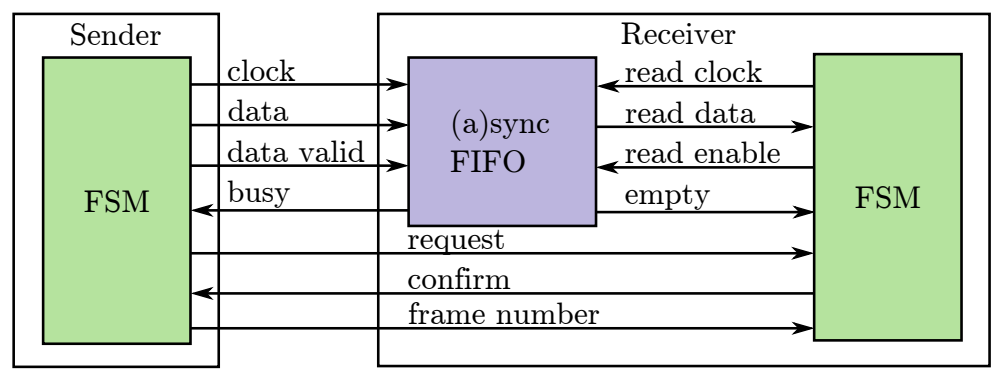

Figure 2.23: Structure of the Pipe communication.

different clock domains, an asynchronous FIFO can be used (see Figure 2.22). In addition to the data and clock signal, the interface uses a "data valid" and a "busy" signal indicating that the data word is valid for this clock cycle, or that the receiver is ready in the next clock cycle.

\subsubsection{Pipe Communication Protocol}

The Pipe communication is an interface for synchronous block transfer. It is similar to the SChannel, but also realizes a frame-based transmission using a frame protocol. The sender starts the handshake by setting the "request" signal to valid. If the receiver now sets the "confirm" signal to valid, the transmission can start in the next clock cycle. The data transmission is realized by SChannel, i. e. is can also operate in asynchronous mode. If the sender wishes to terminate the transfer, then it sets the "request" signal to invalid. The transfer is complete, if the receiver sets the "confirm" signal to invalid. Furthermore, the interface has a signal called "frame number" which can be used to identify a frame or to synchronize different interfaces. Figure 2.23 show the structure of the Pipe communication. An example for a module using an input and output Pipe will be shown in Section A.2.

\subsection{The CCSDS 122.0-B-1 Image Compression Standard}

The CCSDS is a multi-national organization for standardization of space data systems. Since the CCSDS 122.0-B-1 Image Data Compression [24, 133] standard recommendation represents a good trade-off between compression performance and implementation 
complexity, it was selected as basis algorithm for this thesis. It is a wavelet-based image data compression algorithm and often just called CCSDS-IDC ${ }^{28}$. This standard can compress images in lossless as well as in lossy mode. As denoted in [24], the standard differs from JPEG2000 in several aspects:

- it specifically targets high-rate instruments used on board of spacecraft;

- compression performance has been traded off against complexity, with particular emphasis on spacecraft applications;

- the lower complexity of the recommendation supports fast and low-power hardware implementation;

- it has a limited set of options, supporting its successful application without in-depth algorithm knowledge.

In the development phase of the standard, it was a basic requirement that if "any patents were included in the Recommendation, a royalty-free license could be offered to all CCSDS Member Agencies" [25, p. 6-1]. In contrast to methods such as JPEG2000, EZW or SPIHT, no critical patents are currently known that could hinder an implementation. Fundamental techniques used in the standard are embedded coding, bit-plane coding, DPCM, and Colomb-Rice coding.

In the following sections, CCSDS 122.0-B-1 is presented. Subsection 2.4.1 gives on overview on the principle structure of the algorithm. Subsection 2.4 .2 depicts the wavelet transform, Section 2.4.3 shows hierarchy of wavelet coefficient formed by the DWT module and the grouping of wavelet coefficients into blocks and segments, and Section 2.4.4 introduces the entropy coding algorithm used.

\subsubsection{Structure of the Compressor}

A CCSDS 122.0-B-1 encoder is a transform encoder. It consists of two parts: the DWT module and the bit-plane encoder (BPE) (see Figure 2.24). CCSDS 122.0-B-1 is a single band compression technique, i. e. the number of spectral channels that can be encoded is one. Thus, spectral decorrelation of the input data is not performed. At first, the DWT module applies a three-level two-dimensional DWT on the input image. Two specific wavelet filters are provided by the standard: The $\operatorname{CDF}(9 / 7)$ wavelet transform called "Float DWT" and a reversible integer approximation of that transform called "Integer DWT". The Float DWT is intended to be used for lossy compression, whereas lossless compression can only be achieved with the reversible Integer DWT. In the case of the Float DWT, limited precision of the coefficients' floating point representation and a conversion to the nearest integer after transform lead to some loss of information. Lossy compression performance is significantly worse when using the Integer DWT, but in practice, this approach would have the advantage that the output bit-stream is lossless

${ }^{28}$ image data compression (IDC) 


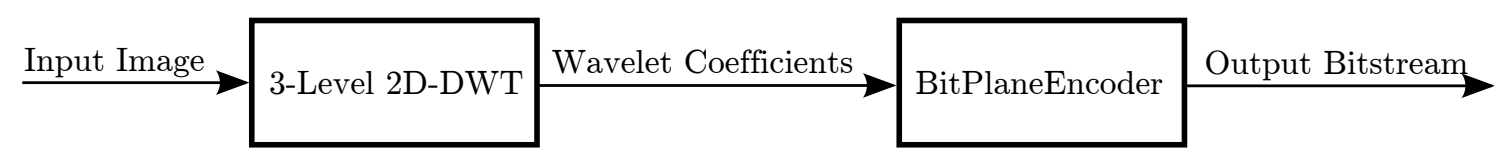

Figure 2.24: Overview on the structure of a CCSDS 122.0-B-1 Encoder.

at the maximum bit rate.

The dynamic range of the input image data is $R$ bit for $R \leq 16$ bit. Signed and unsigned integers are supported. The application of the decomposition procedure to be described in Section 2.4.3 requires that the image dimensions are integer multiples of eight. This - if necessary - is achieved by padding columns and subsequently padding rows to the input image (copying the last column or row). Furthermore, the minimum input image width and height is $17 \mathrm{px}$. As denoted in [24], "Implementation schemes and image reconstruction methods are not part of the Recommended Standard [...]" and are not provided by the CCSDS.

\subsubsection{Wavelet Transform Stage}

As already mentioned, the standard uses either the Float DWT or the Integer DWT. Both transforms are separable and apply the column transform after the row transform (see Section 2.2.2.5). The process of a three-level two-dimensional DWT decomposition of an image is shown in Figure 2.18. In the first level, the input image is decomposed into the sub-bands $\mathrm{LL}_{1}, \mathrm{HL}_{1}, \mathrm{LH}_{1}$, and $\mathrm{HH}_{1}$. In the second level, the result of the sub-band $\mathrm{LL}_{1}$ is again decomposed into the sub-bands $\mathrm{LL}_{2}, \mathrm{HL}_{2}, \mathrm{LH}_{2}$, and $\mathrm{HH}_{2}$. In the third level, the result of the sub-band $\mathrm{LL}_{2}$ is decomposed into the sub-bands $\mathrm{LL}_{3}, \mathrm{HL}_{3}, \mathrm{LH}_{3}$, and $\mathrm{HH}_{3}$.

The analysis filter coefficients of the Float DWT differ from the coefficients already presented in Table 2.1. The reason is that the low-pass filter coefficients are scaled by a factor of $-\frac{1}{\sqrt{2}}$ and the high-pass coefficients are scaled by a factor of $\sqrt{2}$ (see [24, p. 3-3]). The Integer DWT of a signal $x=\left\{x_{0}, x_{1}, x_{2}, \ldots, x_{2 N-1}\right\}$ with $N>2$ is as follows:

$$
\begin{aligned}
D_{0} & =x_{1}-\left\lfloor\frac{9}{16}\left(x_{0}+x_{2}\right)-\frac{1}{16}\left(x_{2}+x_{4}\right)+\frac{1}{2}\right\rfloor \\
D_{j} & =x_{2 j+1}-\left\lfloor\frac{9}{16}\left(x_{2 j}+x_{2 j+2}\right)-\frac{1}{16}\left(x_{2 j-2}+x_{2 j+4}\right)+\frac{1}{2}\right\rfloor \quad \text { for } j=1, \ldots, N-3 \\
D_{N-2} & =x_{2 N-3}-\left\lfloor\frac{9}{16}\left(x_{2 N-4}+x_{2 N-2}\right)-\frac{1}{16}\left(x_{2 N-6}+x_{2 N-2}\right)+\frac{1}{2}\right\rfloor \\
D_{N-1} & =x_{2 N-3}-\left\lfloor\frac{9}{8} x_{2 N-2}-\frac{1}{8} x_{2 N-4}+\frac{1}{2}\right\rfloor \\
C_{0} & =x_{0}-\left\lfloor-\frac{D_{0}}{2}+\frac{1}{2}\right\rfloor \\
C_{j} & =x_{2 j}-\left\lfloor-\frac{D_{j-1}+D_{j}}{4}+\frac{1}{2}\right\rfloor \quad \text { for } j=1, \ldots, N-1
\end{aligned}
$$


where $D_{j}$ denotes the high-pass wavelet coefficients, and $C_{j}$ denotes the low-pass wavelet coefficients. Note that a symmetrical extension of the signal $x$ is implicitly considered by these formulas.

\subsubsection{Block Decomposition Stage}

The DWT module forms a hierarchy of wavelet coefficients:

DC The DC coefficients refer to the coefficients of the $\mathrm{LL}_{3}$ sub-band. The DC coefficients are a representation of the original image at the lowest resolution.

Parents The coefficients in sub-bands $\mathrm{HL}_{3}, \mathrm{LH}_{3}$, and $\mathrm{HH}_{3}$ are called parent coefficients.

Children The coefficients in sub-bands $\mathrm{HL}_{2}, \mathrm{LH}_{2}$, and $\mathrm{HH}_{2}$ are called children coefficients.

Grandchildren The coefficients in sub-bands $\mathrm{HL}_{1}, \mathrm{LH}_{1}$, and $\mathrm{HH}_{1}$ are called grandchildren coefficients.

A block is a group of one DC coefficient and the 63 corresponding $\mathrm{AC}$ coefficients (3 parents, 12 children, 48 grandchildren). A block loosely represents a region in the input image. Figure 2.25 shows this relationship. For the BPE, the blocks are further arranged into groups: A segment is a group of $S$ consecutive blocks, where $16 \leq S \leq 2^{20}$. Segments are encoded independently and are further partitioned into gaggles, which is a group of $G=16$ consecutive blocks. The last segment or gaggle of an image can contain less than $G$ blocks. The horizontal and vertical number of blocks of a transformed image are

$$
\mathrm{S}_{x}=\left\lfloor\frac{I_{w}+7}{8}\right\rfloor
$$

and

$$
\mathrm{S}_{y}=\left\lfloor\frac{I_{h}+7}{8}\right\rfloor
$$

where $I_{w}$ and $I_{h}$ are the number of columns and rows in the image. In strip-mode, the number of blocks in a segment is $S=\mathrm{S}_{x}$. In frame mode, the number of blocks in a segment is $S=\mathrm{S}_{x} \cdot \mathrm{S}_{y}$.

\subsubsection{Coding Stages}

Once the coefficient are grouped into segments, the BPE can start to encode the image. Figure Figure 2.26 shows the program and data flow of the BPE [25].

\subsubsection{Preparation Steps}

Two steps have to be done before the coding: 


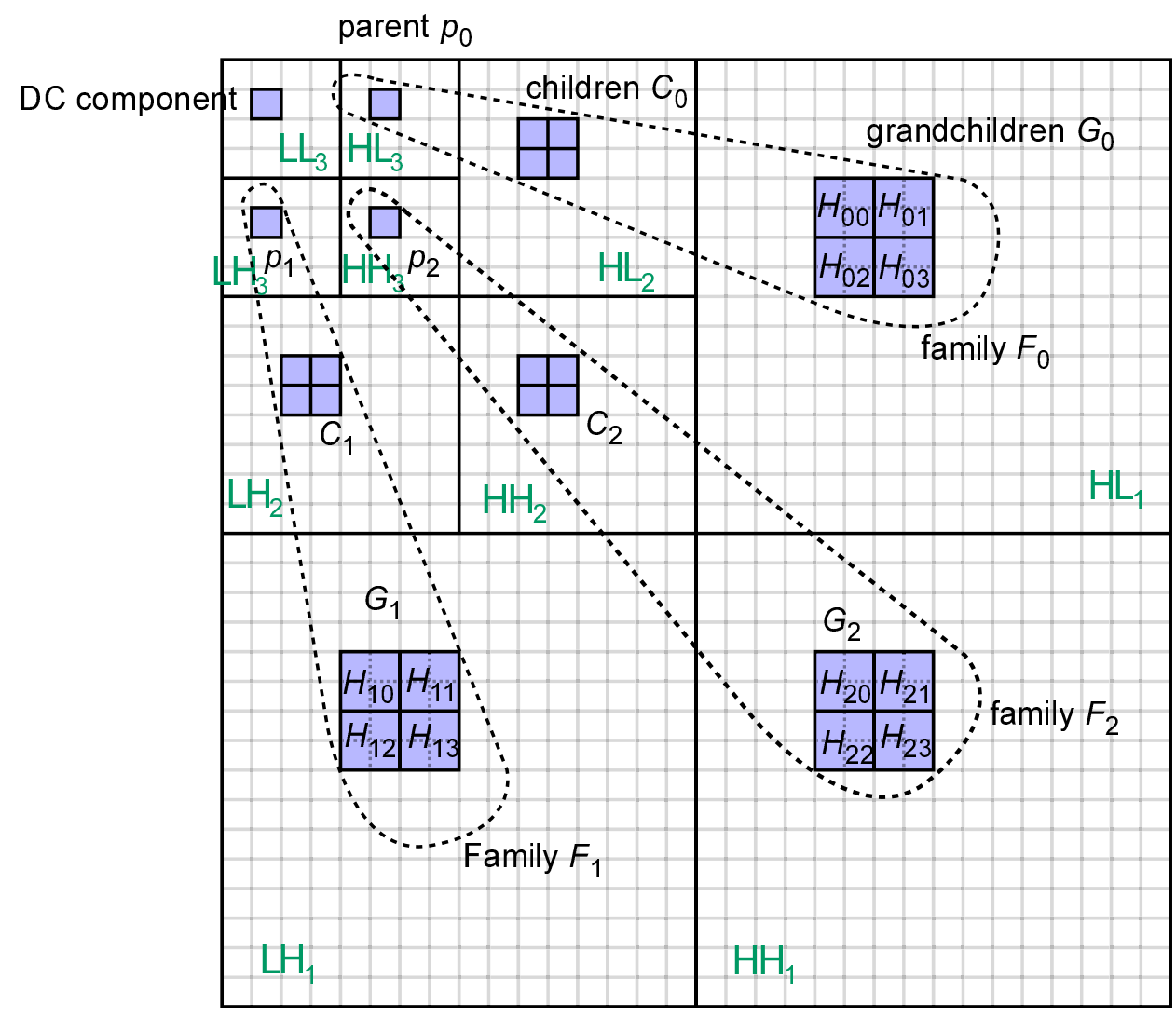

Figure 2.25: A block consisting of a DC coefficient and 63 AC coefficients [24, p. 4-1].

1. Weight the coefficients if the Integer DWT is used: This is necessary to optimize the rate distortion [25, p. E-1 ff.]. Since the sub-band weights have been obtained empirically, the standard supports user-defined weights. Every sub-band has its own weighting factor $w$ and the coefficients of each sub-band are multiplied by $2^{w}$ (shifted left by w bits).

2. Determining of the bit depth of the DC coefficients of a segment BitDepthDC and the bit depths of the $\mathrm{AC}$ coefficients for each Block $m$ referred to as BitDepthAC ${ }_{m}$. The DC coefficients are coded in two's complement representation. Thus, the number of bits necessary to code a DC coefficient $c_{m}$ is as follows:

$$
\operatorname{BitDepthDC}\left(c_{m}\right)= \begin{cases}1+\left\lceil\log _{2}\left|c_{m}\right|\right\rceil, & c_{m}<0 \\ 1+\left\lceil\log _{2}\left(1+c_{m}\right)\right\rceil, & \text { otherwise }\end{cases}
$$

The AC coefficients are coded in sign-magnitude representation. Thus, the number of bits necessary to code an AC coefficient $d_{m}$ is as follows:

$$
\operatorname{BitDepthAC}\left(d_{m}\right)=\left\lceil\log _{2}\left(1+\left|d_{m}\right|\right)\right\rceil
$$

Finally, maximum values for the DC and AC coefficients' bit-depth are determined over the entire segment and denoted as BitDepthDC and BitDepthAC. 


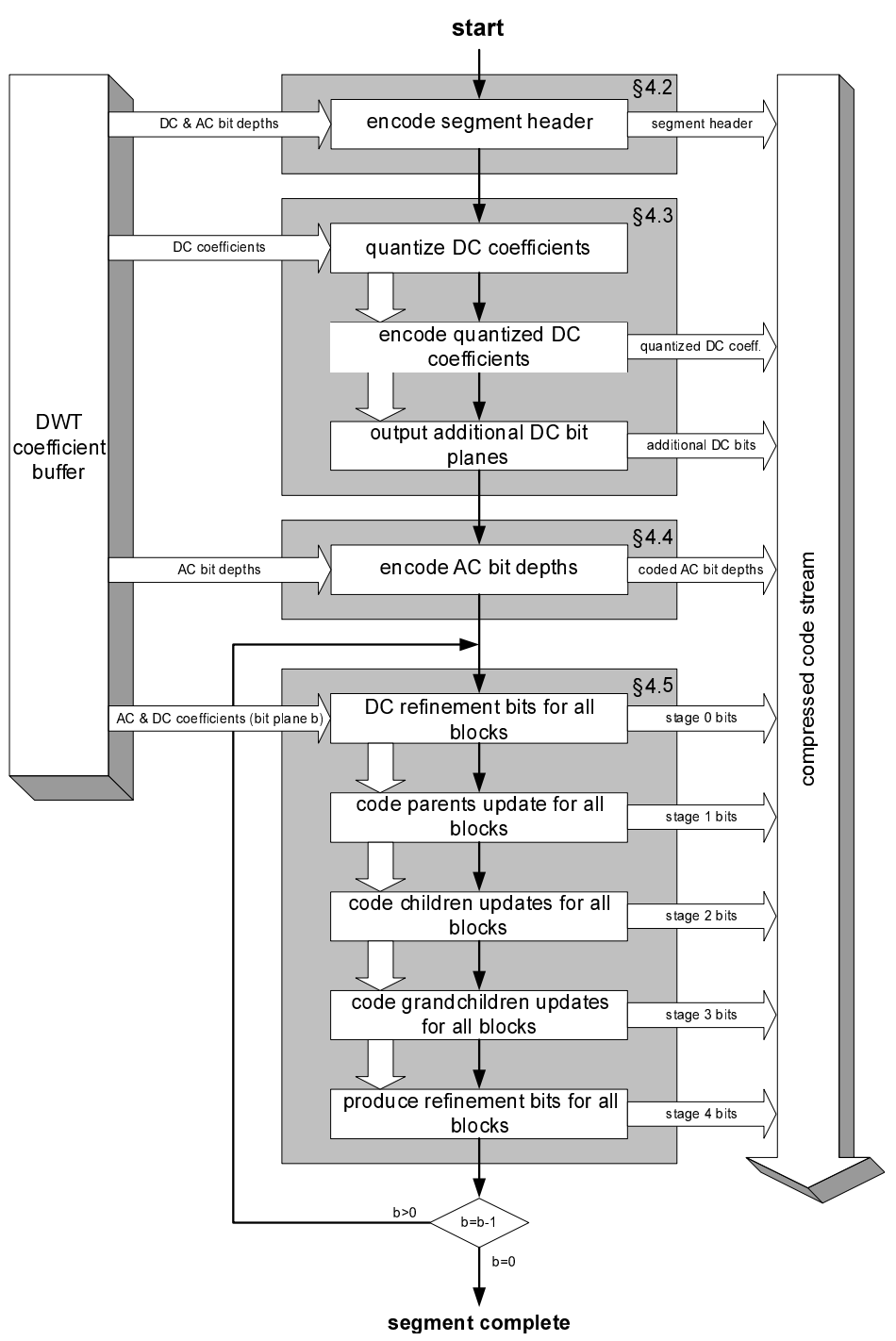

Figure 2.26: Program and data flow of the BPE [25, p. 2-12].

BitDepthAC $\mathrm{C}_{m}$ denotes the number of bits necessary to code any $\mathrm{AC}$ coefficient in a block $m$.

\subsubsection{Segment Header}

Each segment starts with a segment header containing information about the current segment. It consists of mandatory and optional parts. The mandatory part contains flags for the first and last segment of an image, the BitDepthDC and BitDepthAC, a segment counter, and indicates which optional header parts are included. See [24, pp. 4-5ff] for details about the segment header. 


\subsubsection{Initial Coding}

In the initial coding stage, the DC coefficients are quantized and coded. Then, optionally, some additional DC bit-planes are coded. At the end, BitDepthAC $\mathrm{A}_{m}$ will be coded for each block $m^{29}$.

The DC coefficients $c_{m}$ are quantized with a quantization factor $q$ :

$$
c_{m}^{\prime}=\left\lfloor\frac{c_{m}}{2^{q}}\right\rfloor
$$

The quantization factor $q$ depends on the wavelet transform type and on the dynamic range of the wavelet coefficients:

$$
q^{\prime}= \begin{cases}0 & \text { BitDepthDC } \leq 3 \\ \text { BitDepthDC }-3 & \text { BitDepthDC }-(1+\lfloor\text { BitDepthAC } / 2\rfloor) \leq 1 \text { and BitDepthDC }>3 \\ \text { BitDepthDC }-10 & \text { BitDepthDC }-(1+\lfloor\text { BitDepthAC } / 2\rfloor)>10 \text { and BitDepthDC }>3 \\ 1+\lfloor\text { BitDepthAC } / 2\rfloor & \text { otherwise }\end{cases}
$$

and

$$
q=\max \left(q^{\prime}, \operatorname{BitShift}(L L 3)\right),
$$

where BitShift $(L L 3)$ denotes the weighting factor of sub-band $\mathrm{LL}_{3}$. The number of bits necessary to represent each quantized DC coefficient $c_{m}^{\prime}$ is as follows:

$$
N=\max (\text { BitDepthDC }-q, 1)
$$

In a next step, DPCM is applied on the quantized DC coefficients $c_{m}^{\prime}$ :

$$
\delta_{m}^{\prime}= \begin{cases}c_{m}^{\prime} & m=0 \\ c_{m}^{\prime}-c_{m-1}^{\prime} & \text { otherwise. }\end{cases}
$$

and

$$
\delta_{m}= \begin{cases}2\left(\delta_{m}^{\prime}\right) & 0 \leq \delta_{m}^{\prime}=\leq \theta_{m} \\ 2\left|\delta_{m}^{\prime}\right|-1 & -\theta_{m} \leq \delta_{m}^{\prime}<0 \\ \theta_{m}+\left|\delta_{m}^{\prime}\right| & \text { otherwise, }\end{cases}
$$

where $\theta_{m}=\min \left(c_{m-1}^{\prime}-x_{\min }, x_{\max }-c_{m-1}^{\prime}\right), x_{\min }=2^{N-1}$, and $x_{\max }=2^{N-1}-1$. DPCM is followed by Rice Coding [24, p. 4-19ff]. After all quantized DC coefficients are encoded and depending on $q$ and BitDepthAC, some additional DC bit-planes may be refined [24, section 4.3.3]. The next step is to encode BitDepthAC ${ }_{m}$ (bitdepth of the $\mathrm{AC}$ coefficients of block $m$ ) with the same DPCM method already used for the quantized DC coefficients.

\footnotetext{
${ }^{29}$ The blocks $\S 4.3$ and $\S 4.4$ are referred to herein as initial coding.
} 


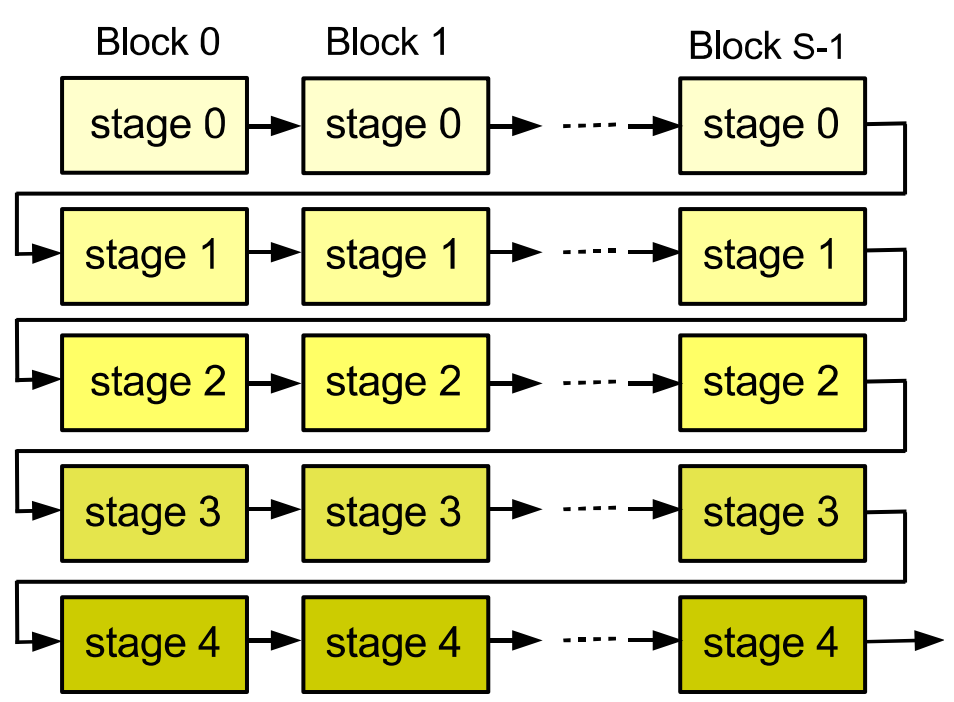

Figure 2.27: Overview of the stage coding withing a segment [24, p. 4-4].

\subsubsection{Bitplane Coding}

The BPE encodes the wavelet coefficients, as the name suggests, bit-plane-wise starting with bit-plane $b=$ BitDepthAC -1 and in decreasing order. Within a bit-plane $b$, the coding process is divided into 5 stages (stages $0-4$ ). In stage 0 , remaining bits of the DC coefficients are coded (DC refinement). Stage 1-3 encode the AC coefficients' sign and the position of the significant bit. The significant bit is the highest non-zero bit. A coefficient is selected if the significant bit is encoded. Once an AC coefficient is selected, Stage 4 encodes the AC coefficients' refinement. The type $t_{b}(x)$ of coefficient $x$ depends on the current bit-plane index $b$ and the position of the first non-zero bit (see [24, p. 4-26]):

$$
t_{b}(x)= \begin{cases}-1 & b<\operatorname{BitShift}(\operatorname{subband}(x)) \\ 0 & |x|<2^{b} \\ 1 & 2^{b} \leq|x|<2^{b+1} \\ 2 & 2^{2+1} \leq|x|\end{cases}
$$

Here, BitShift(subband $(x)$ ) denotes the number of bits shifted during the quantization step. Stage 1 refers to the refinement of the parents coefficients. When a new bit-plane is encoded, the type of each AC coefficient is determined. The same procedure is applied to the children coefficients at stage 2 and to the grandchildren coefficients at stage 3 . If a coefficient's type is 1 , i. e. it is selected, its sign is coded in stages 1,2 or 3 . At the next bit-plane, its type will be 2 or -1 . At stage 4 , the bits of all AC coefficients of type 2 are encoded. Stages 1-3 produce words which are first mapped to symbols. The symbols are then encoded with variable-length code (VLC). All bits of a stage are written to the output bit-stream before the next stage is commenced, even though the optimal code for the VLC is determined by the stage 1 to 3 . 
The procedure of coding the stages 1,2 , and 3 is well explained in [24, pp. 4-27 ff] and is summarized below (Figure 2.27). The entropy coding process defines three new groups of coefficients (see also Figure 2.25):

$$
P=\left\{p_{0}, p_{1}, p_{2}\right\} \quad D_{i}=\left\{C_{i}, G_{i}\right\} \quad B=\left\{D_{0}, D_{1}, D_{2}\right\}
$$

A shorthand notation is used for certain binary words:

- $\operatorname{types}_{b}[\Psi]$ denote the binary word consisting of the $b^{\text {th }}$ magnitude bit of each coefficient $x$ in $\Psi$ such that $t_{b}(x)$ equals 0 or 1 .

- $\operatorname{signs}_{b}(\Psi)$ denote the binary word consisting of the sign bit of each coefficient $x$ in $\Psi$ such that $t_{b}(x)=1$, with a sign bit of " 1 " for negative coefficients and " 0 " for non-negative coefficients.

- Given a list of type values $\Lambda=\left\{\lambda_{0}, \lambda_{1}, \lambda_{2}, \ldots, \lambda_{l}\right\}$, let tword $[\Lambda]$ denote the binary word consisting of the sequence of type values $\lambda_{i}$ in $\Lambda$ that are equal to 0 or 1.

The BPE uses the following transition words to indicate when groups of coefficients are all of type 0 :

- $\operatorname{tran}_{B}= \begin{cases}\text { null } & \text { if } \operatorname{tran}_{B}=1 \text { at any more significant bit plane } \\ \operatorname{tword}\left[\left\{t_{\max }(B)\right\}\right] & \text { otherwise. }\end{cases}$

- $\operatorname{tran}_{D}=\operatorname{tword}\left[\left\{t_{\max }\left(D_{i}\right): i=0,1,2\right.\right.$, such that $t_{\max }\left(D_{i}\right) \neq 1$ in all more significant bit planes\}]

- $\operatorname{tran}_{G}=\operatorname{tword}\left[\left\{t_{\max }\left(G_{i}\right): i=0,1,2\right.\right.$, such that $t_{\max }\left(D_{i}\right)>0$ in current or any more significant bit planes $\}]$

- $\operatorname{tran}_{H i}=\operatorname{tword}\left[\left\{t_{\max }\left(H_{i 0}\right), t_{\max }\left(H_{i 1}\right), t_{\max }\left(H_{i 2}\right), t_{\max }\left(H_{i 3}\right)\right\}\right]$ for $i=0,1,2$.

As already mentioned, bit-plane coding operates on bit-planes $b=$ BitDepthAC 1 , BitDepthAC $-2, \ldots, 0$. At bit-plane $b$ the following sequence of words are generated for each block of a segment:

Stage 1 (parents): $\operatorname{types}_{b}[P], \operatorname{signs}_{b}[P]$

Stage 2 (children):

1. $\operatorname{tran}_{B}$

2. $\operatorname{tran}_{D}$, if $\operatorname{tran}_{B} \neq 0$ and $t_{\max }(B) \neq-1$

3. types $b\left[C_{i}\right]$ and $\operatorname{signs}_{b}\left(C_{i}\right)$ for each $i$ such that $t_{\max }\left(D_{i}\right)>0$

Stage 3 (grandchildren): If $\operatorname{tran}_{B}=0$ or $t_{\max }(B)=-1$, then stage 3 is unnecessary and shall be omitted. Otherwise:

1. $\operatorname{tran}_{G}$ 
2. $\operatorname{tran}_{H i}$, for each $i$ such that $t_{\max }\left(G_{i}\right)>0$

3. types ${ }_{b}\left[H_{i j}\right]$ and $\operatorname{signs}_{b}\left[H_{i j}\right]$ for each $i$ such that $t_{\max }\left(G_{i}\right)>0$ and each $j$ such that $t_{\max }\left(H_{i j}\right)>0$

Words corresponding to signs, i. e. $\operatorname{signs}_{b}[P], \operatorname{signs}_{b}\left(C_{i}\right)$, and $\operatorname{signs}_{b}\left[H_{i j}\right]$ and those having a length of less than 2 bits are directly written to the output bit-stream. All other words are entropy encoded with VLC as follows:

- The words are mapped to symbols, see [24, p. 4-30]

- An optimal code option is determined for a group of symbols and each symbol is encoded using a variable-length binary codeword, see [24, p. 4-31]

The standard specifies that "for each word size within a gaggle, the code option selected is the one that minimizes the encoded length of the gaggle when all words are coded" (see [24, p. 4-32]). The code option identifier is written immediately before a symbol with the corresponding length is written. It is a little unclear, about which set of symbols the optimal code option is determined. According to the CCSDS reference compressed files supplied in [26], the optimal code option is determined for each gaggle and for stages 1 , 2 , and 3 .

\subsection{Summary}

This chapter has presented the fundamentals and related work of optical remote sensing using satellites, wavelet-based image data compression and hardware architectures for DWT and on-board image compression.

Fundamentals of optical remote sensing are necessary for understanding and analyzing the top-level requirements on the new image data compression architecture. Terms like GSD, ground track speed, line rate, and parameters of optical sensor systems are introduced. Next, basic concepts of image data compression that are necessary to understand the underlying compression algorithm. Hardware architectures for DWT are necessary for an efficient implementation of the DWT. Knowledge about hardware architectures for on-board image data compression is needed to compare and evaluate the proposed architecture. The standard interfaces of the hardware operating system are used for internal and external connections of the proposed architecture.

The CCSDS 122.0-B-1 algorithm is the basis for the architecture presented in this thesis. First the structure of the compression algorithm was presented. The algorithm uses a three-level two-dimensional wavelet transform for spatial decorrelation and an entropy encoder that exploits the hierarchy of the wavelet coefficients to efficiently encode the image data. The algorithm was explained by presenting the mathematical background. Based on the CCSDS 122.0-B-1 algorithm, this thesis will propose extensions that can be used for ROI coding and interactive transmission of the image data. Furthermore, an architecture will be presented that can be used for FPGA implementation. 



\section{Extensions of the CCSDS 122.0-B-1 Image Compression Standard}

The CCSDS 122.0-B-1 standard presented in Section 2.4 does not support an a-posteriori change of compressing parameters that can be used for ROI extraction. Because the encoder generates an embedded bit-stream, the significance of each bit or the position of a block, segment or image region inside the bit-stream can only be determined by decoding. There is no possibility to determine a particular unit of information inside the bit-stream. Furthermore, the CCSDS 122.0-B-1 standard does not support ROI coding, multi-spectral compression or spectral decorrelation. However, as already depicted, the algorithm is well suited for real-time image compression on-board spacecraft. In this chapter, an approach to extend the CCSDS 122.0-B-1 standard called bit-stream scalability will be presented and analyzed. Scalability here means that the compressed image or parts of it can be re-assembled to achieve a particular image quality, spatial or spectral resolution in any area of an image without the need for re-coding. The method will also be useful to efficiently build transfer frames for MS encoded data.

This chapter is arranged as follows: Section 3.1 discusses possibilities to realize ROI coding in combination with CCSDS 122.0-B-1. Section 3.2 presents a concept to achieve scalability of the compressed bit-stream. An approach for multi-spectral compression is presented in Section 3.3. It also uses the scalability approach for rate-distortion optimization. A short summary of this chapter is given in Section 3.4.

\subsection{Region-Of-Interest Coding}

The basic idea of ROI coding is to encode regions of interest with low distortion (lossless) and other regions with higher distortion (lossy). A ROI mask contains the information about whether a certain region is of interest or not. It is convenient to adjust the granularity of the ROI mask to a unit of information used in the compression algorithm.

Figure 3.1 shows an example using ROI coding for the test image "marstest". The integer wavelet and a segment size of $S=16$ was used. The lossless compressed image (without ROI) has a size of 157,363 bytes $(4.8023 \mathrm{bpp})$. A segment size of $S=16$ results in 256 segments. The inner 64 segments where chosen as ROI. For the non-ROI segments, the DCStop option was set. The resulting image size is 41,534 bytes (1.2675 bpp). Figure 3.1c shows the absolute difference image between Figures 3.1a and 3.1b. The area where the compression is lossless is highlighted with white color. 


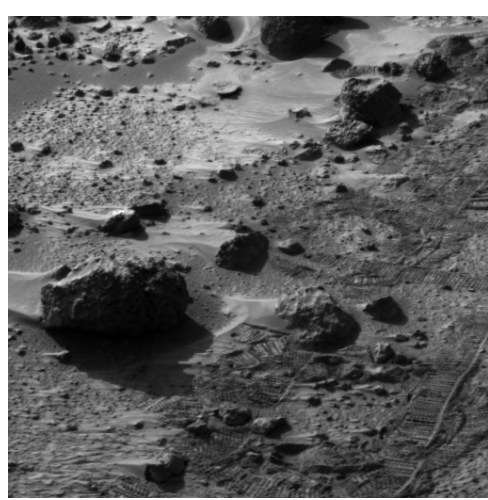

(a) original image

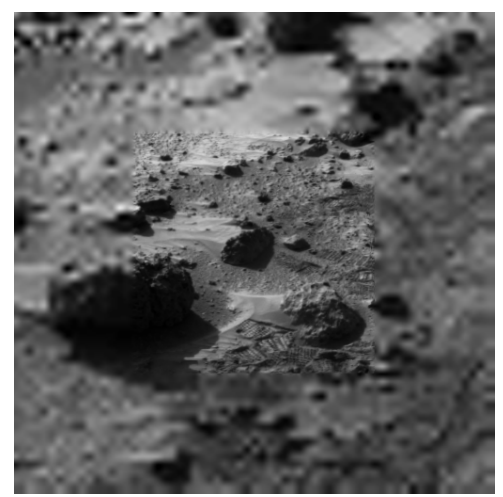

(b) with centered ROI

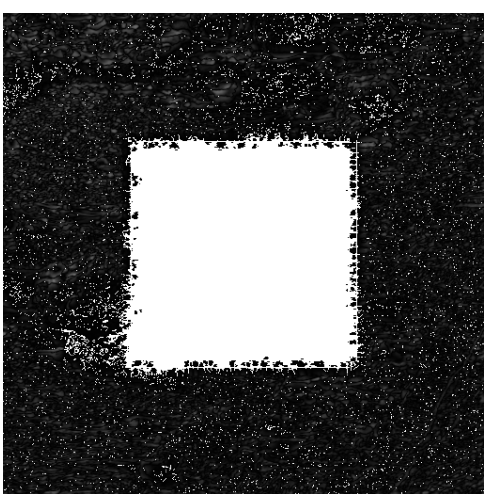

(c) difference image

Figure 3.1: An example using ROI coding for the test image marstest.

For this thesis, it is assumed that the ROI masks are either transferred via Telecommand (TC) before image acquisition, or are determined with image classifiers (e.g. cloud detection). Note that the architecture presented in Chapter 4 does not implement any online classification algorithms.

\subsubsection{Unit of Information}

In CCSDS 122.0-B-1, units of information can be formed as follows: The coarsest unit consists of the image itself. The finest unit may be a block of 64 coefficients from the 10 sub-bands or a coefficient itself. As explained in Section 2.4.3, an image is divided into segments, gaggles and blocks.

Controlling the compression parameters for a complete image is straightforward to implement and produces no additional overhead, since the compression parameters are transmitted anyway. However, a scene must be split into multiple images to realize ROI support. This results in discontinuities at the image borders and a reduced compression efficiency (see Figure 3.2). Furthermore, the ROI cannot be modified without re-coding (and splitting) the image. Using a segment as ROI unit is easy to implement, since only a header update must be transmitted in subsequent segments. The ROI granularity can be controlled by the segment size $S$, but can only be changed on segment level. If a gaggle or a block is used as finest ROI unit, the ROI granularity is high or very high, and can be controlled on gaggle or block level, respectively. In both approaches, the encoder needs to be adjusted in order to transmit the ROI mask for every gaggle or block, which produces a significant overhead resulting in a worse compression efficiency. On the other hand, it would be possible to change the ROI on a very localized level. Table 3.1 summarizes the advantages and disadvantages of using these units for ROI coding. 
Table 3.1: Comparison of using image-, segment-, gaggle- or block-based ROI coding.

\begin{tabular}{|c|c|c|}
\hline & Advantages & Disadvantages \\
\hline Image & $\begin{array}{l}\text { - easy to implement } \\
\text { - no overhead }\end{array}$ & $\begin{array}{l}\text { - scene must be split into multiple } \\
\text { images } \\
\text { - coding efficiency suffers } \\
\text { - discontinuities at the image } \\
\text { borders } \\
\text { - ROI cannot be changed after } \\
\text { compression }\end{array}$ \\
\hline Segment & $\begin{array}{l}\text { - easy to implement } \\
\text { - ROI granularity can be } \\
\text { controlled by segment size }\end{array}$ & $\begin{array}{l}\text { - ROI granularity can be changed } \\
\text { only on segment level } \\
\text { - little overhead (additional } \\
\text { header for subsequent segments) }\end{array}$ \\
\hline Gaggle & $\begin{array}{l}\text { - ROI granularity is high } \\
\text { - ROI granularity can be changed } \\
\text { on gaggle level }\end{array}$ & $\begin{array}{l}\text { - high effort to change ROI after } \\
\text { compression } \\
\text { - encoder must be adapted } \\
\text { - high overhead (parameters for } \\
\text { every gaggle) }\end{array}$ \\
\hline Block & $\begin{array}{l}\text { - ROI granularity is very high } \\
\text { - ROI granularity can be changed } \\
\text { on block level }\end{array}$ & $\begin{array}{l}\text { - very high effort to change ROI } \\
\text { after compression } \\
\text { - encoder must be adapted } \\
\text { - very high overhead (parameters } \\
\text { for every block) }\end{array}$ \\
\hline
\end{tabular}

For image-based ROI, the number of regions is obviously equal to one. The number of regions for segment-based $\mathrm{ROI}$ is

$$
R_{s}=\left\lceil\frac{I_{w}}{8}\right\rceil \cdot\left\lceil\frac{I_{h}}{8}\right\rceil \cdot \frac{1}{S}
$$

where $I_{w}$ and $I_{h}$ denote the width and the height of the image and $S$ denotes the segment size in blocks. For gaggle-based ROI, the number of regions is

$$
R_{g}=\left\lceil\frac{I_{w}}{8}\right\rceil \cdot\left\lceil\frac{I_{h}}{8}\right\rceil \cdot \frac{1}{G}
$$

where $G$ denotes the size of a gaggle in blocks. Usually, $G=16$ and $G \leq S$.

Segment-based ROI will be pursued in this thesis, since it provides high flexibility and causes only a little overhead in the implementation as well as in the transmission. Since compression efficiency increases with the segment size and ROI granularity decreases with the segment size, a trade-off between compression efficiency and ROI granularity 


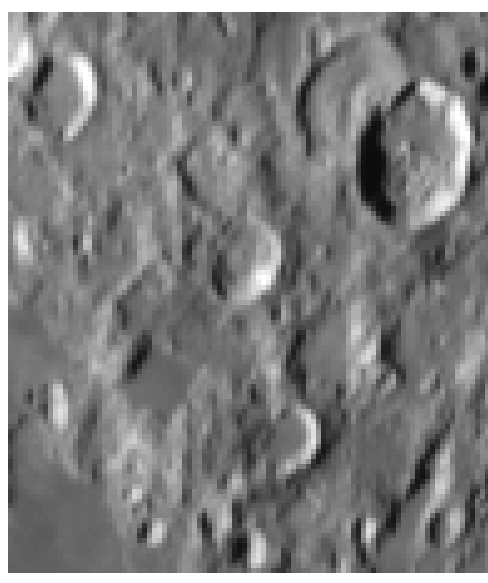

(a) Original image parts

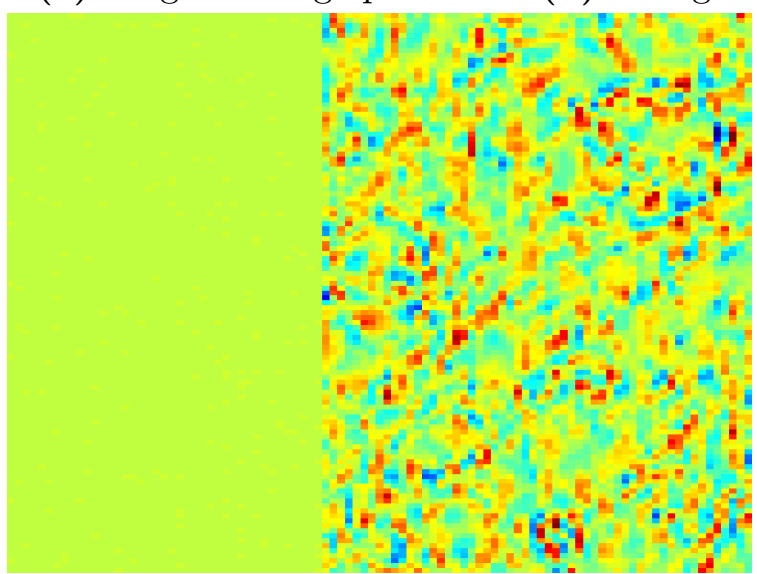

(d) Difference between (a) and (b)

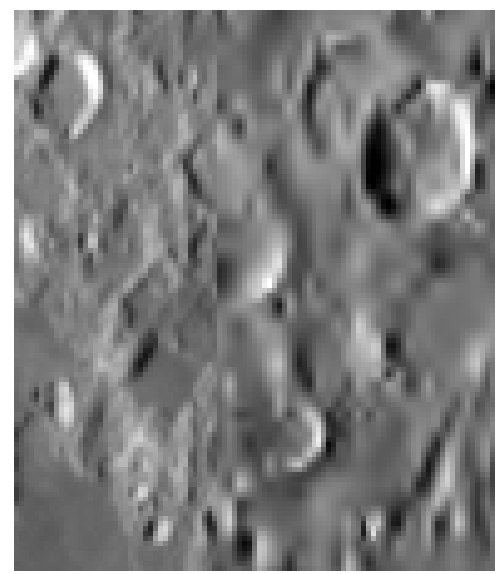

(b) Tiling in spatial domain

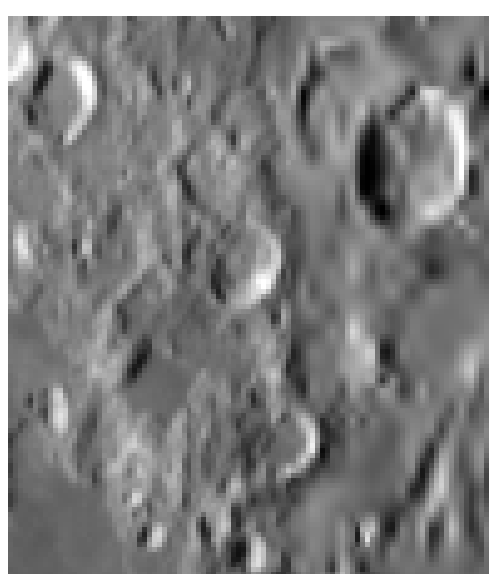

(c) Tiling in wavelet domain

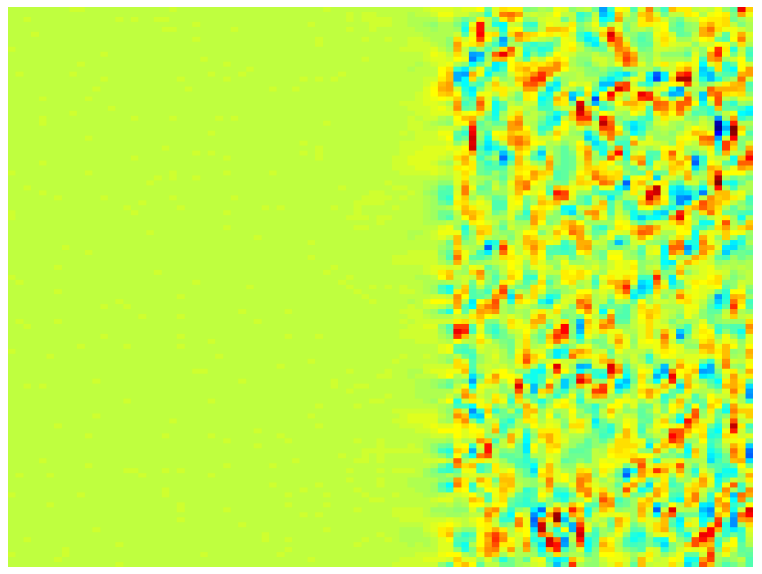

(e) Difference between (a) and (c)

Figure 3.2: Discontinuities at the image borders when tiling is applied in spatial domain. The image shows a region of the lunar image (Figure B.3).

has to be found. Figure 3.3 shows the rate-distortion curves for two different images for several segment sizes $S$. A segment size of 16 or 32 blocks causes much higher distortions than larger segment sizes. The CCSSD 122.0-B-1 standard recommends a segment size of $S=128$. In this thesis, a segment size of $S=64$ blocks and $S=128$ blocks seems to be a good choice in terms of compression efficiency and resource requirements. Smaller segments will decrease the compression efficiency whereas larger segments will increase the memory requirements for segment encoding (will be analyzed in Section 4.3).

\subsubsection{Partitioning Schemes}

Since a segment combines consecutive blocks of an image, the segments form horizontal "stripes" of blocks. The compression algorithm operates in stripe mode, if the segment size $S$ is $\left\lceil\frac{I_{w}}{8}\right\rceil$. In this mode, a segment consists of $\left\lceil\frac{I_{w}}{8}\right\rceil$ consecutive blocks in the horizontal and one block in the vertical direction. 

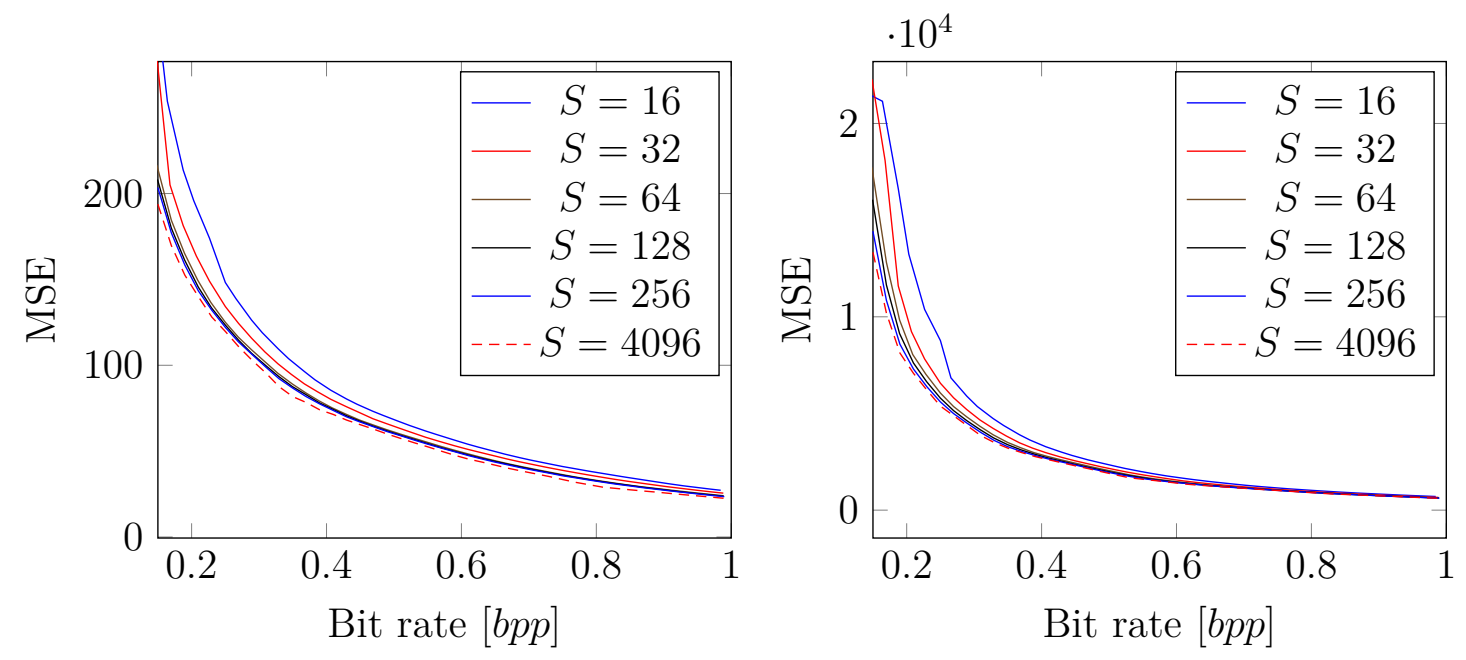

Figure 3.3: Rate-distortion curve for different segment sizes (left: marstest; right: pleiadesportdebouc-pan, upper-left 4096 blocks).

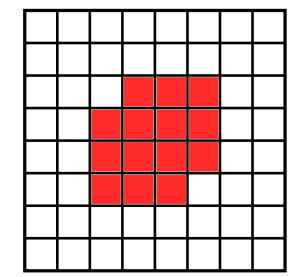

(a) $S=1$

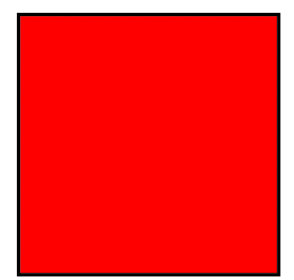

(b) $S=64$

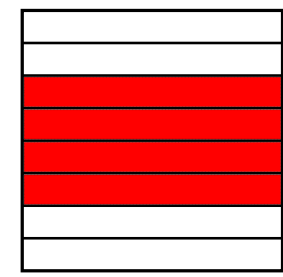

(c) $S=8$

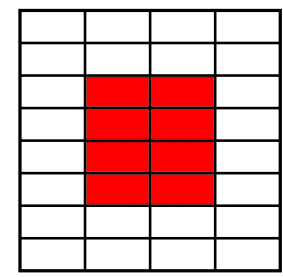

(d) $S=2$

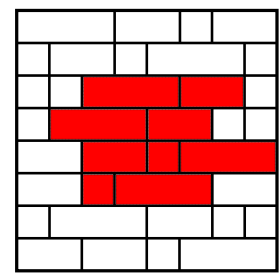

(e) $S$ variable

Figure 3.4: Different segment sizes for an image of $64 \times 64 \mathrm{px}(8 \times 8$ blocks $)$ and an exemplary region-of-interest.

There is a high number of possible partitioning schemes, since the segment size can be changed for each segment. Some possible schemes for an image with $64 \times 64 \mathrm{px}$ are shown in Figure 3.4. The minimum number of segments is given by the maximum segment size $S_{\max }$ and the image width $I_{w}$ and height $I_{h}$ :

$$
\text { \#Segments } s_{\min }=\left\lceil\frac{\left\lceil I_{w} / 8\right\rceil \cdot\left\lceil I_{h} / 8\right\rceil}{S_{\max }}\right\rceil
$$

The maximum number of segments is equal to the number of blocks and can be achieved if the maximum segment size $S_{\max }$ is set to 1 .

$$
\text { \#Segments } s_{\max }=\left\lceil I_{w} / 8\right\rceil \cdot\left\lceil I_{h} / 8\right\rceil
$$

\subsection{Scalability}

As already mentioned, bit-stream scalability means that the compressed image or parts of it can be re-assembled to achieve a particular image quality, spatial or spectral 


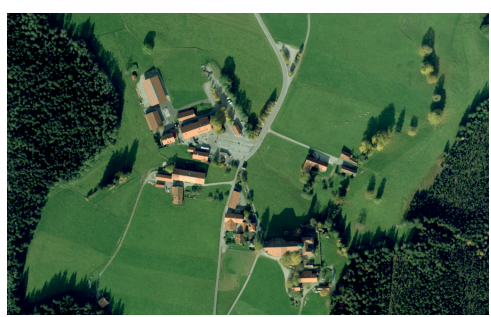

(a) Original image

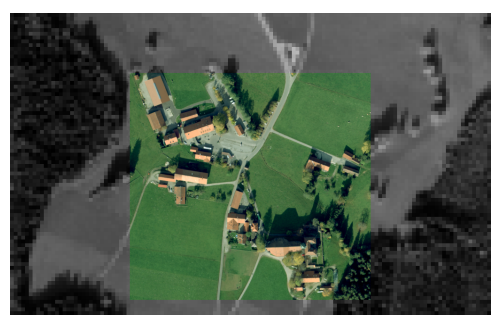

(b) Stored image

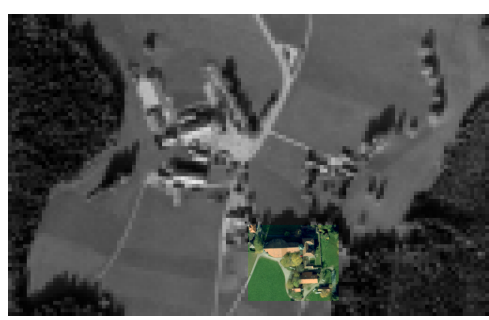

(c) Downloaded image

Figure 3.5: The same image at three different stages: a) before compression, b) after compression and c) as requested by the ground station.

resolution and ROI support without the need for re-coding, while the image may have been compressed lossless. Scalability is achieved by

- modifying the compression algorithm in a way that different spatial or spectral regions of an image can be independently decoded,

- creating an index of the encoded bit-stream during compression so that the position of an image block is known.

Scalability allows to compress images with a region-specific spatial or spectral resolution based on a mask which is available during compression, and to assemble a transfer frame which contains an even coarser spatial or spectral resolution, i. e. to change the compression parameters after compression without re-coding. Figure 3.5 shows an example with three images: Figure 3.5a contains the original image before compression. Figure 3.5b shows a stored image, if the image compression module has used ROI-specific compression parameters (e.g. resolution, number of channels, etc.). If the ground station requests a more localized ROI, more details may be omitted. Figure 3.5c shows an example where the ROI is smaller.

A similar approach to extend the CCSDS 122.0-B-1 standard is presented in [41]. The authors define a so-called Minimal Unit of informaTion (MUT), which can be decoded independently. To achieve this, embedded headers able to locate the MUTs are proposed. They are located at the beginning of each MUT and contain its length. A modification of the DPCM algorithm, used to encode the quantized DC coefficients and the AC coefficients' bit-depth, is proposed, so that gaggles can be decoded independently. Note that gaggles are also used in stages $1-3$ of the bit-plane coding process. The article does not explain how scalability is achieved in stages 0 and 4 . Furthermore, some implementations details are not explained.

\subsubsection{Analysis of the Compression Parameters}

The parameters of the compression algorithm shown in Table 3.2 have a direct influence on the distortion within a segment. SegByteLimit can be either zero for a non-size-limited segment or non-zero for a size-limited segment. This value is relevant for the decoder to 
detect the end of a segment, since the concatenated segments of an image form the output bit-stream. The encoder considers the parameter when accomplishing a certain output bit rate. UseFill indicates whether the segment should be filled with zeros in order to reach the desired segment size. DCstop, BitPlaneStop and StageStop are algorithm-specific termination parameters that are used for quality control.

Table 3.2: Quality-related compression parameters of CCSDS 122.0-B-1 [24].

\begin{tabular}{|c|c|}
\hline Parameter & Standard's description \\
\hline SegByteLimit & Maximum number of compressed bytes in a segment. \\
\hline UseFill & $\begin{array}{l}\text { Specifies whether fill bits will be used to produce SegByteLimit } \\
\text { bytes in each segment. }\end{array}$ \\
\hline DCstop & $\begin{array}{l}\text { Indicates whether compressed output stops after coding of } \\
\text { quantized DC coefficients. }\end{array}$ \\
\hline $\begin{array}{l}\text { BitPlaneStop/ } \\
\text { StageStop }\end{array}$ & $\begin{array}{l}\text { When DCStop }=0 \text {, indicates limit on coding of DWT coefficient } \\
\text { bit-planes. When BitPlaneStop }=b \text { and StageStop }=s \text {, compressed } \\
\text { output stops once stage } s \text { of bit plane } b \text { has been completed, unless } \\
\text { coding stops earlier in the segment because of the segment byte } \\
\text { limit (SegByteLimit). }\end{array}$ \\
\hline
\end{tabular}

\subsubsection{Length and Size of the Resulting Bit-Stream Parts}

The approach presented here allows changing quality-related compression parameters after coding. As shown in Section 2.4, a compressed image is composed of several parts:

- Header

- Quantized DC coefficients

- DC coefficients refinement (additional bit-planes, optional)

- AC coefficients' bit-depths

- Bit-planes from stage coding (stages 0-4)

Independent of the termination parameters, the segment header and the quantized DC coefficients must be encoded. Otherwise, no information about certain image regions can be decoded. If DCstop is set, coding stops after these steps. Otherwise, the encoder proceeds with the DC coefficient refinement, which depends on BitDepthAC, the DC quantization factor $q$, and the sub-band weights.

The length of the header is 3 to 20 bytes depending on which parts of the header are selected for coding. The length of the quantized and encoded DC coefficients $L_{d c}$ depends on the image content and the segment size $S$. The average numbers of bits necessary to 


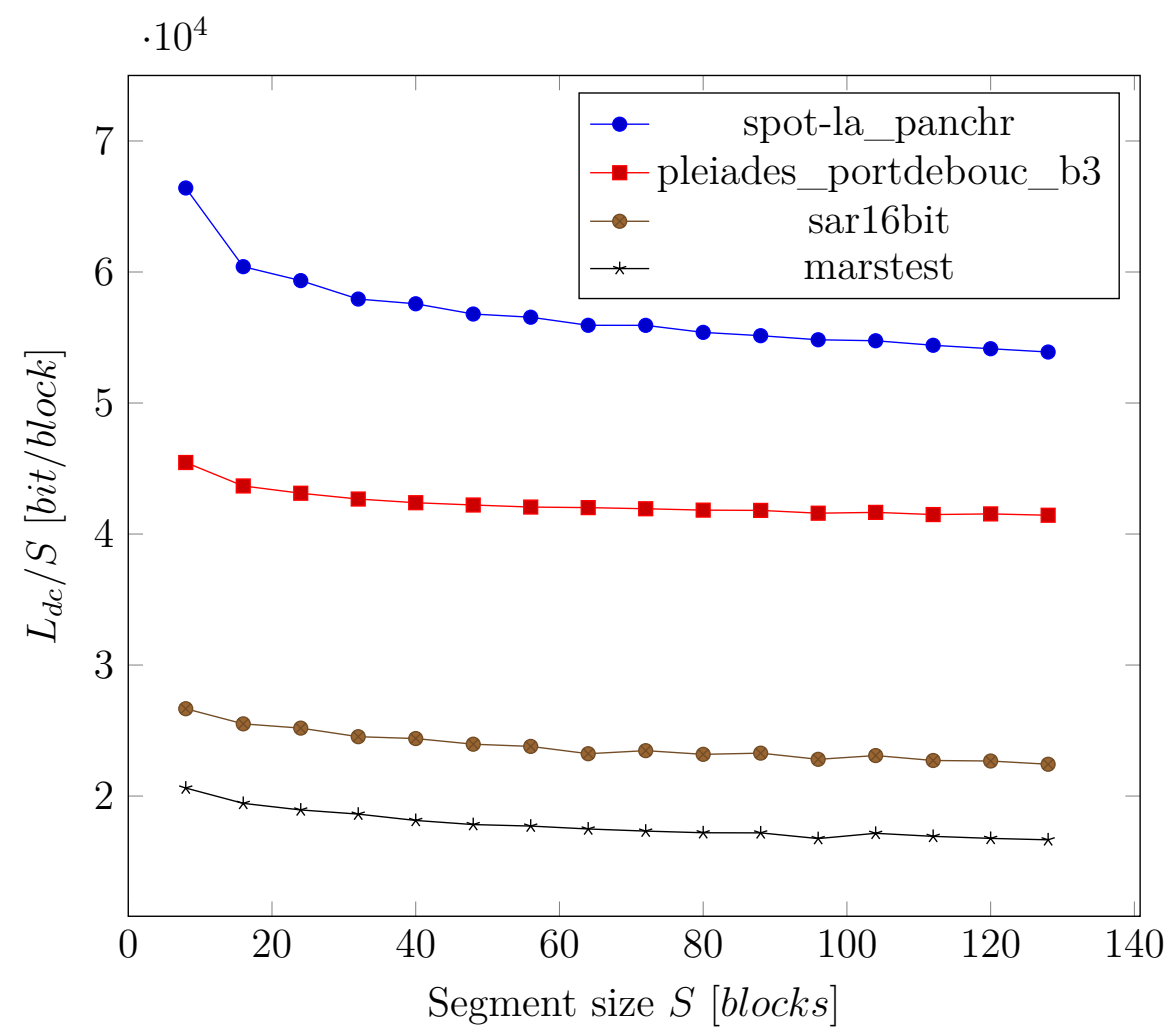

Figure 3.6: Average size of the encoded quantized DC coefficients for some selected images.

encode the quantized DC coefficients for a selection of images are shown in Figure 3.6. Note that the coding efficiency rises with the segment size $S$. It can be seen that a segment size of $S=64$ or $S=128$ is a good choice in terms of compression efficiency.

If the image contains small high-frequency components, additional DC coefficient bits must be encoded: If $q>\max \{\operatorname{BitDepthDC}$, Weight $(L L 3)\}$, some of the most significant bits of each DC coefficient are encoded. The number of bits written in this stage is

$$
L_{d c r}=S \cdot(q-\max \{\operatorname{BitDepthDC}, \operatorname{Weight}(L L 3)\})
$$

This rarely occurs in natural images. In fact, results show that none of the reference images presented in Appendix B cause the encoder to produce DC coefficients refinement bits. For testing, DC coefficients refinement encoding can be provoked by the compression of an image that has previously been compressed with DCstop=1.

The bit-depth of the AC coefficients is written if DCstop $=0$. The encoder uses the same $\mathrm{DPCM} /$ rice coding scheme as for the quantized DC coefficients. The number of bits written in this stage depends on the segment size $S$ and the dynamic range of the image. It is typically $2-3$ bits/block (see Figure 3.7 ). 


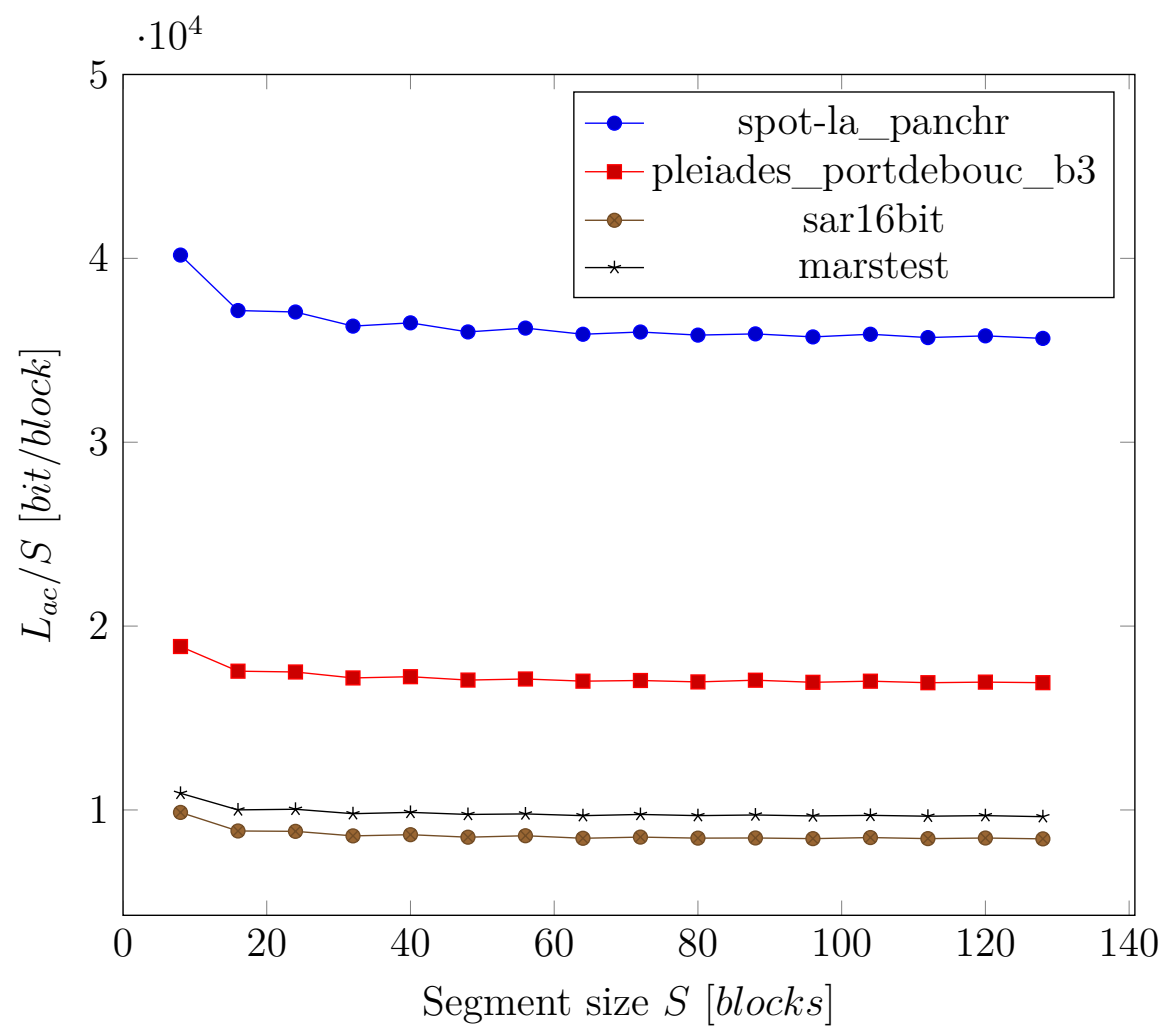

Figure 3.7: Average size of the encoded bit-depth of the AC coefficients for some selected images.

The allocation of bits to each component of the compressed bit-stream is shown in Figures 3.8a and 3.8b. The major part of the compressed bit-stream is comprised of bits from stage coding, especially from stage $2-4$. Stage coding operates on bit-planes $b=$ BitDepthAC -1 , BitDepthAC $-2, \ldots, 0$ and for each bit-plane on stages $0-4$. Stage 0 contains the DC coefficient's refinement. Stages 1, 2 and 3 encode the sign and the position of the significant bit of the corresponding AC coefficients. Stage 4 contains AC refinement bits of all coefficients (parents, children and grand children). If BitPlaneStop $=b$, the encoder stops after bit-plane $b$. If StageStop $=s$, the encoder stops after stage $s+1$. The default parameters are BitPlaneStop $=0$ and StageStop $=3$.

\subsubsection{Bit-Stream Indexing}

To support scalability and the multi-spectral interleaving scheme presented in Section 3.3.2, several positions inside the compressed bit-stream are stored in an index table. The index table contains the position of the first byte of the segment (header) and the lengths of each subsequent element.

Since the header and the quantized DC coefficients are encoded in any case, they both get an entry in the index table. If $\mathrm{DCstop}=1$, coding stops at this position and no 


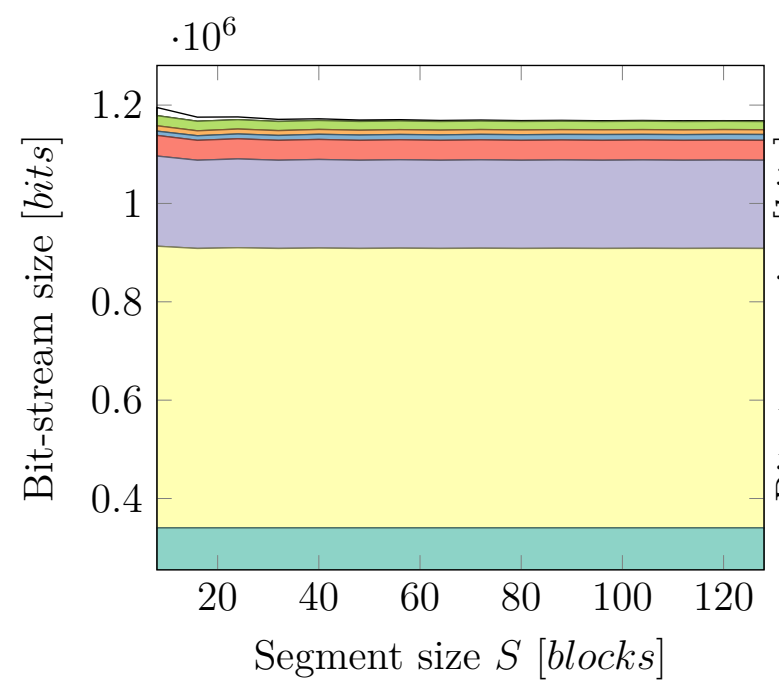

(a) marstest

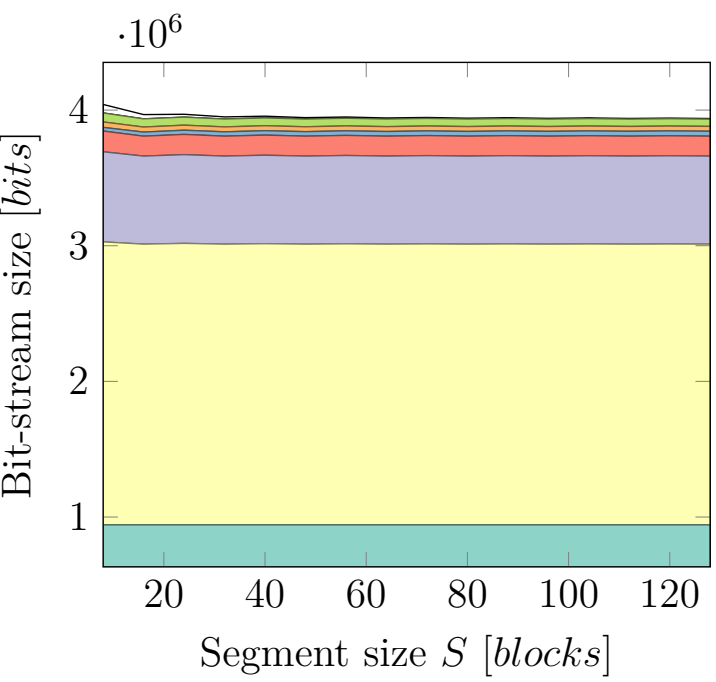

(b) spot-la-panchr

\begin{tabular}{|cccccc|}
$\square$ & Header \\
Stage 1 & $\square$ & Quantized DC & $\square$ & AC BitDepth & \\
Stage 2 & $\square$ & Stage 3 & Stage 0 \\
& & Stage 4 \\
\hline
\end{tabular}

Figure 3.8: Distribution of individual bits within the compressed bit-stream for some images

further entries will be inserted into the index table. If $L_{d c r}>0$, the index table contains one entry for the DC coefficients refinement. If DCstop $=0$ and BitDepthAC $>0$, the index table contains the length of the encoded AC coefficients' bit-depth $L_{a c}$. To enable scalability, the individual parts of the compressed bit-stream for each bit-plane and stage must be separable. This is straightforward for stages 0 to 4 , since once a stage is present, its content is not affected by BitPlaneStop or StageStop. An entry in the index table after each stage is used to separate the stage bits. The maximum number of index entries $N_{\text {all }}$ is given by the following equation:

$$
N_{\text {all }}(\text { BitDepthAC, StageStop })=2+1+1+\operatorname{BitDepthAC} \cdot(\text { StageStop }+2)
$$

For example, assuming BitDepthAC $=24$ and StageStop $=3$, the maximum number of index entries is $N_{\max }=4+24 \cdot 5=124$. Listing 3.1 shows a pseudo-code of a possible implementation of the index structure. The actual number of segments and bit-planes might reduce the size of the index table. Note that the index table is not embedded in the bit-stream and must not be transferred to a ground station (see below). The size of the index table is investigated in Section 5.2.

Listing 3.1: Data structure of the index table.

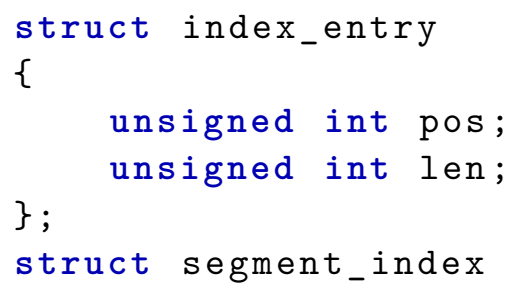




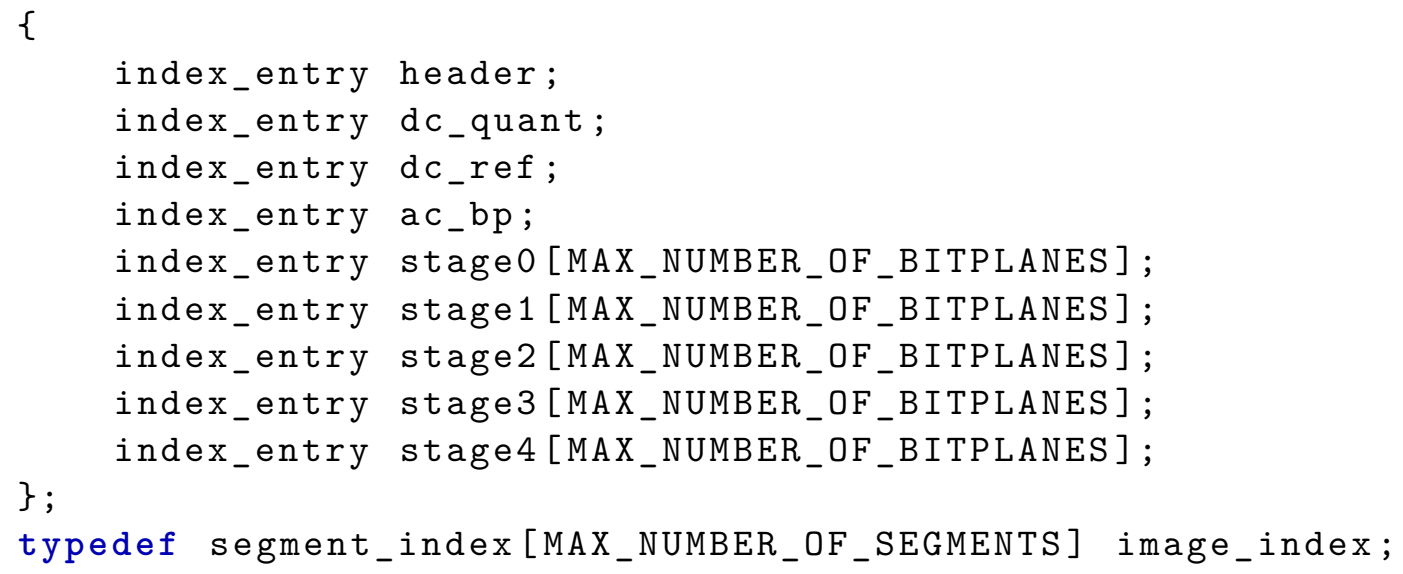

Additional embedded headers are not necessary if the bit-stream is transferred to the ground station without manipulation or re-assembling. Furthermore, the ground station typically knows the configuration of the received data packets, as they have been requested by the ground station. A further advantage of bit-stream indexing is that it can be used to detect and handle bit-stream errors. Usually, Reed-Solomon coding is used to detect and correct multiple random bit-stream errors. For example, if an error cannot be corrected, the index can be used to localize and restrict the impact of the error to a localized spatial region of the image.

\subsubsection{Bit-Stream Re-Assembling}

The index table can be used for on-board manipulation or re-assembling the compressed bit-stream. The index itself does not need to be transmitted to the ground station.

If an image is first requested by the ground station, any quality-related compression parameter can be adapted before transmission. The request of the ground station starts an on-board re-assembling process that uses the bit-stream and the index table stored in the on-board mass memory. This process can be performed, for example, by a CPU. The resulting bit-stream will be transferred to the ground station. It may include an ROI, however, it can be decoded without the index, since it complies with the CCSDS 122.0-B-1 standard, and thus requires only the embedded headers. Figure 3.9 illustrated this approach.

In a second (or later) step, it is conceivable that the ground station requests an update for an already transmitted (overview) image. The on-board re-assembling process is nearly identical to the first step, except that only additional information will be transferred. Only details for some specific regions are transmitted. The ground station can merge this update packet with the previously transferred image during decoding, since it knows which parts of the bit-stream can be found in which transmission packet. Depending on the implementation of the decoder, it is also possible to re-use already decoded parts of the bit-stream, which leads to interactive decoding. This approach in shown in Figure 3.10. 


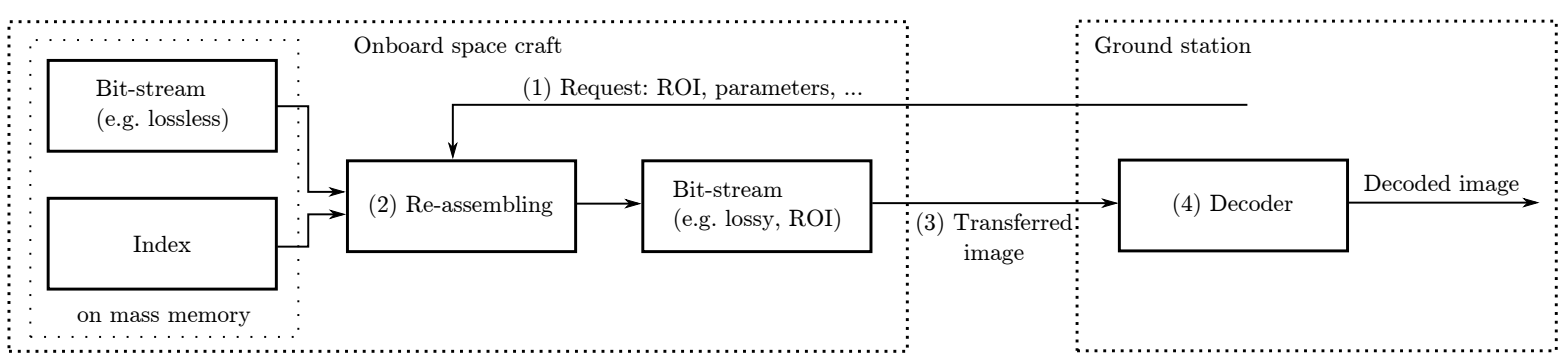

Figure 3.9: On-board re-assembling of the bit-stream. The ground station decodes a single image.

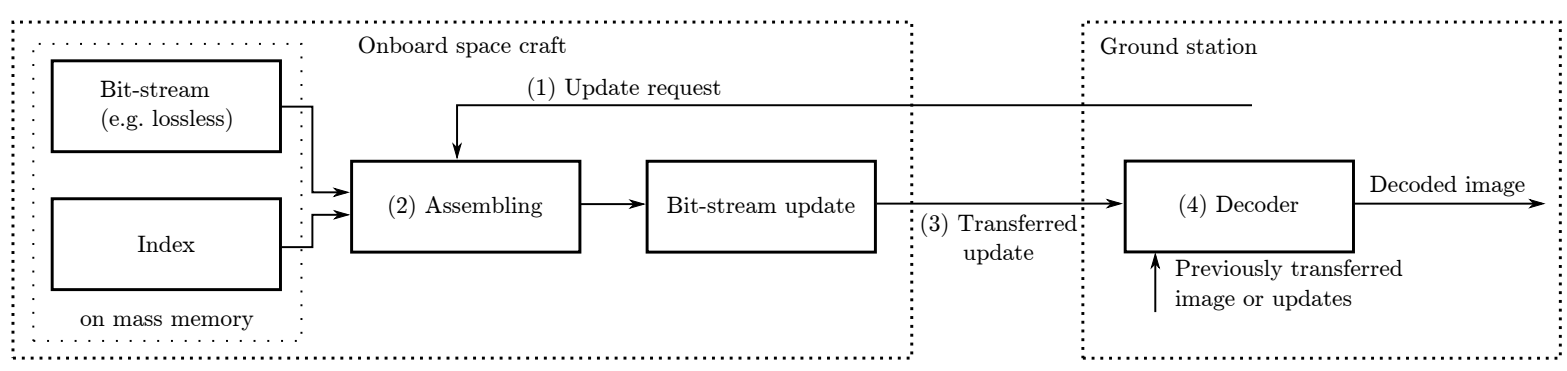

Figure 3.10: On-board assembling of an update for an already transferred image. The ground station decodes the previously transferred image and uses the update to increase the details.

\subsection{Multi-spectral Image Compression}

If an image consists of multiple spectral channels, it is desirable to consider the spectral correlation in order to optimize the compression result. However, in the simplest case, multi-spectral channels are compressed independently, i. e. spectral decorrelation is not performed and multiple entropy encoders run independently with a constant target bit rate. Figure 3.11 shows this approach. Albeit it is the simplest possibility, this approach has two major disadvantages:

- Spectral correlation is not exploited. This results in a sub-optimal compression efficiency.

- To achieve a certain total output data rate over all spectral channels, a per-channel data rate must be assigned to each of the compressors. Since the entropy of the spectral channels is highly variable, it usually happens that a spectral channel with a low entropy gets a low distortion whereas a channel with a high entropy gets a high distortion.

\subsubsection{Spectral Decorrelation}

As mentioned above, the compression ratio can significantly be improved by using spectral decorrelation. Figure 3.12 shows a possibility to extend every mono-band image compression algorithm for MS compression. In this compression scheme, spectral 


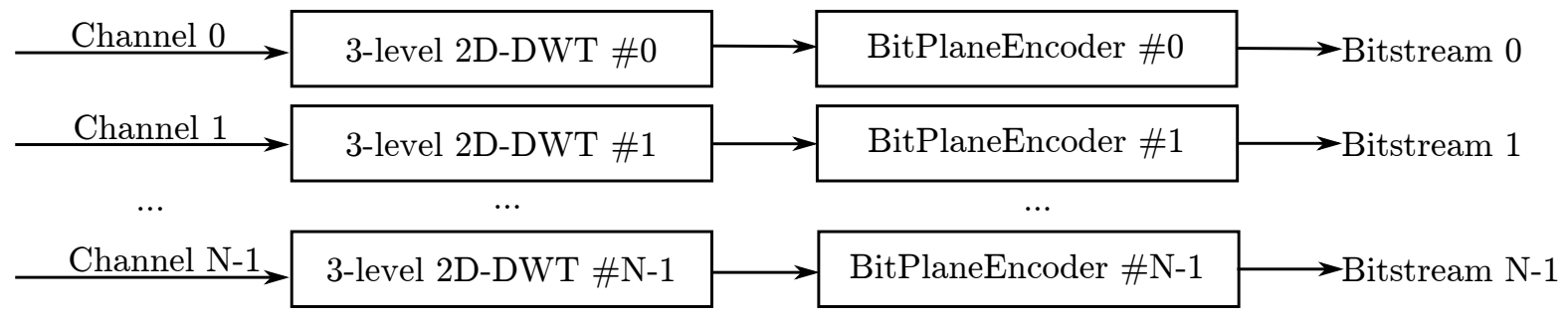

Figure 3.11: Independent processing of several spectral channels. Spectral decorrelation is not used.

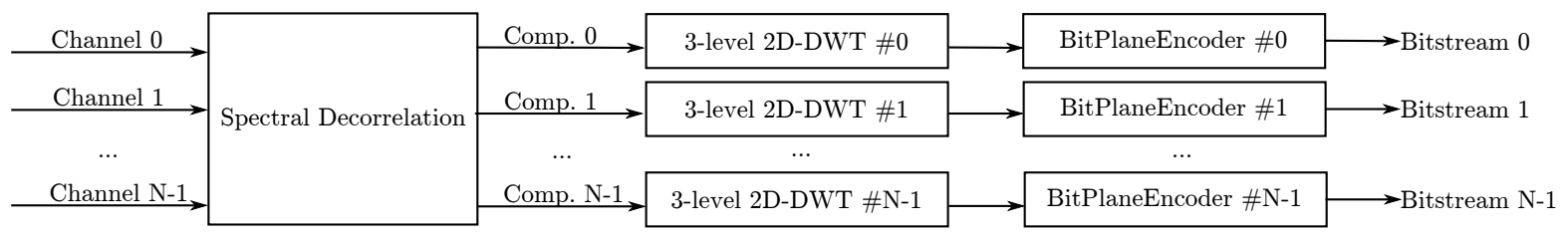

Figure 3.12: The spectral channels are decorrelated and then compressed independently.

decorrelation is performed between all image channels. The number of components corresponds to the number of image channels of the input image. Although this approach exploits the correlation between the individual spectral channels, it has the disadvantage that the bit rate must be individually controlled by the entropy encoders.

As depicted in Section 2.2.1.2 (spectral) decorrelation can be performed by prediction, by transform, or by a filter bank. An optimal spectral decorrelation can be achieved by the Karhunen-Loève transform (KLT). The degree of spectral correlation is evident in the covariance matrix of the image channels and can be expressed with the correlation coefficient $\rho$ as shown in Figure 3.13. Many authors use the principal component analysis (PCA) for spectral decorrelation [29, 31, 32, 36, 41, 93]. However, an implementation is expensive in terms of complexity and execution time. The integer KLT can be used in order to achieve real-time capability and reversibility [58, 68, 84].

Beside its optimum decorrelation, the KLT has decisive disadvantages:

- The covariance matrix of the spectral channels must be calculated. This process is quite complex since it operates region-based and requires multiplications between every corresponding pixel of every spectral channel. Note that hyper-spectral data usually has more than 100 spectral channels.

- The eigenvalues and eigenvectors of the covariance matrix must be calculated.

- The calculated transform must be applied to the input data. 


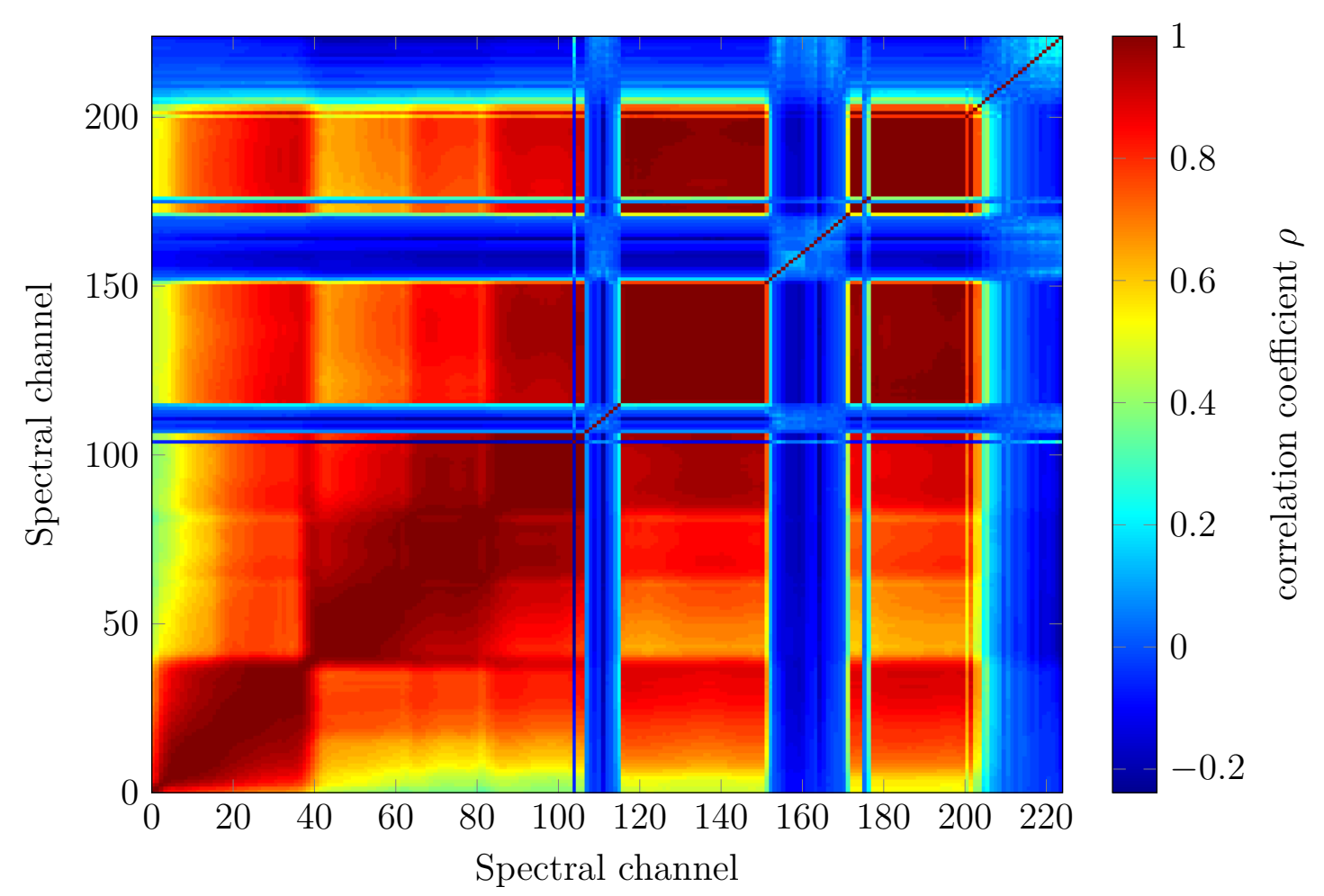

Figure 3.13: Correlation of the 224 spectral channels of aviris scene 0 (cal).

- Since the transformation is specific for each image, it must be transmitted along with the compressed data.

- Using a non-integer transform leads to an irreversible transform, i.e. lossless compression is usually not possible.

Transforms which are usually used for spatial decorrelation can also be used for spectral decorrelation: A DCT is used in [4]. The DWT is used in [42]. In [95], the author used the discrete Hartley transform (DHT). Vector quantization methods are presented in $[87,88,92]$. A DPCM is used in [4]. Fuzzy prediction is used in [9]. Other prediction techniques are presented in [12] and [119].

A good approach to reduce the complexity is to use a static transform matrix for every set of images. The transform matrix can be trained offline. According to [111], the compression ratio gain for registered images is $30-40 \%$ for a specific compression rate. Registered here means that the transform matrix was determined for this or a similar image type. For non-registered images, there is no gain, but the compression performances are not lower than for the non-decorrelation case. 


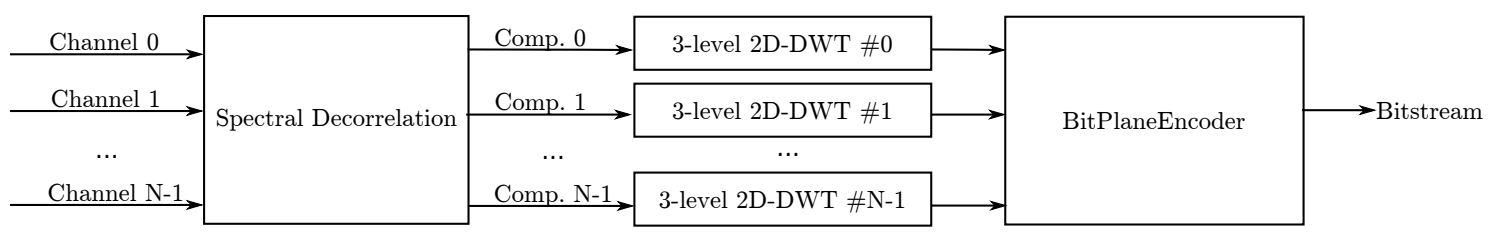

Figure 3.14: The spectral channels are decorrelated and then compressed jointly.

\subsubsection{Multi-Channel Entropy Coding}

In multi-channel entropy coding, one entropy encoder operates on all channels, i. e. the rate-distortion optimization is applied over all channels and not just within the individual channels. Figure 3.14 shows this concept. The three-dimensional data cube is encoded by a single instance of the entropy encoder. The main problem of this approach is that the entropy encoder must be able to process multiple spectral channels at the same time and that the accumulated data rate of the spectral channels might become very high.

The concept of scalability presented in Section 3.2 can be used to effectively add multichannel support to the hardware-accelerated entropy encoder presented in this thesis. In a post-compression step, the bit-streams and the index tables can be used to merge the individual single-channel bit-streams into one multi-channel bit-stream. The merging process uses the index table and goes through all the steps and stages of the CCSDS 122.0-B-1 encoding process and concatenates the corresponding output bits of each image channel. A weighting of the image channels might be necessary and can easily be performed if a spectral decorrelation was performed before compression. Thus, in the case of lossy image compression, rate-distortion optimization can be realized by assigning more bits to image channels with a high entropy than with a lower entropy.

\subsection{Summary}

This chapter has presented extensions of the CCSDS 122.0-B-1 algorithm that are developed for this thesis and are currently not part of the standard. Methods for ROI coding were investigated. It has been shown that controlling the quality-related compression parameters in combination with the segment size is the best solution for ROI coding. The concept of scalability was presented. It allows to re-assemble the compressed bit-stream in order to change quality-related compression parameters. The additional index table itself has no impact on the image quality, but it can be used to transmit certain regions lossless. The concept can be used for interactive transmission or to extract an image with specific ROI from an image that has already been compressed without re-coding. It can also be used for multi-spectral coding by using the index table to create a multi-spectral bit-stream from several individually compressed single-band images. 



\section{A New Image Data Compression Architecture}

In Chapter 1, it was assumed that the increasing storage capacity on-board of a future spacecraft and the developments in radio transmission will hardly compensate the increasing amount of data. A fast and flexible access to the compressed data will be necessary to serve different requests of different ground stations. In Section 2.4, the CCSDS 122.0-B-1 standard for image data compression was presented. In order to develop an architecture that can fulfill the future requirements, extensions of this standard were presented in Chapter 3.

This chapter presents a design of a real-time architecture for satellite image data compression which can be used for various future applications. The architecture is initially developed for a commercial FPGA board, but shall later be implementable on spacequalified hardware. In this chapter we first elicit and analyze the top-level requirements on the new image data compression architecture. Subsequently, a design concept containing sub-components of the architecture will be presented. A requirement analysis and a design of these sub-components will be shown. In Section 4.3, a design concept of the architecture will be presented. A short summary of this chapter is given in Section 4.4.

\subsection{Quantitative Requirements}

The image compression functionality should be implemented directly onto the focal plane assembly (FPA). The basic idea behind that approach is, that the amount of data that is transferred between individual modules should be reduced as early as possible in the signal processing chain in order to reduce the power consumption, the complexity of the architecture's interconnects, and finally the volume and costs. Present interfaces to connect the FPA and the memory management unit (MMU) consume approximately $2 \mathrm{~W}$ for a $1 \mathrm{Gbit} / \mathrm{s}$ interconnect ${ }^{1}$. Supposing a data rate of $8 \mathrm{Gbit} / \mathrm{s}$, a single interconnect will cause a power dissipation of approximately $16 \mathrm{~W}$. Note, that with today's interface technology, an imaginary future system with a data rate of $64 \mathrm{Gbit} / \mathrm{s}$ will have a power dissipation of $128 \mathrm{~W}$ for a single interconnect.

The architecture should support scalability and interactive transmission as described in Chapter 3. Since certain parts of the bit-stream can be individually located, this

\footnotetext{
${ }^{1}$ The maximum power dissipation of a typical radiation hardened serial interface is $2 \mathrm{~W}+1.25 \mathrm{~W}=$ $3.25 \mathrm{~W}$ for $1.575 \mathrm{Gbit} / \mathrm{s}[7,8]$
} 


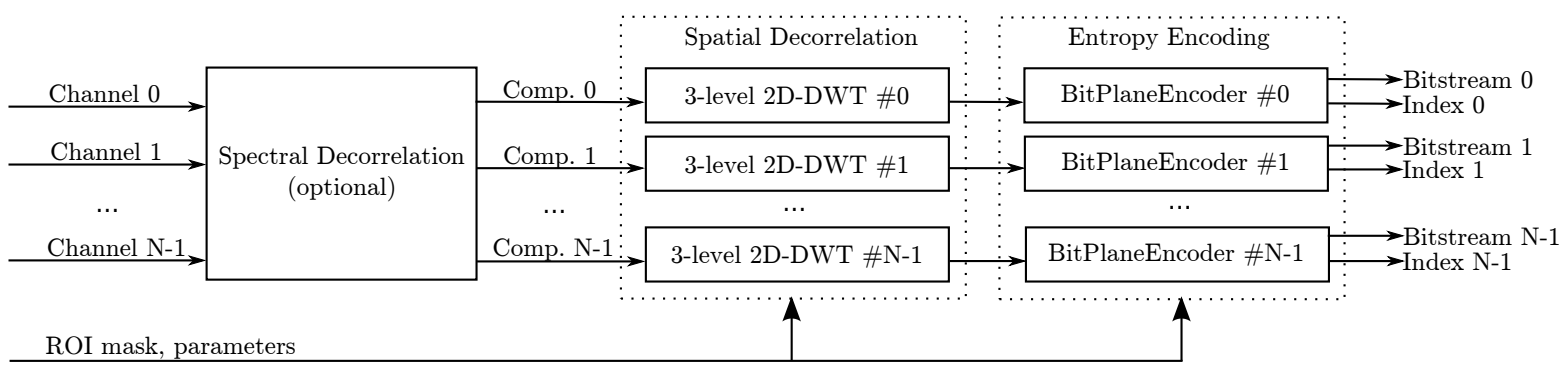

Figure 4.1: Overview on the proposed architecture for multi-spectral compression.

will also allow to change quality-related compression parameters after compression. If multi-spectral data is to be compressed, spatial decorrelation and entropy coding of multi-spectral image data can be executed logically independent between the channels. A simple but effective spectral decorrelation should be used to reduce inter-band correlation. Scalability will also allow to optimize the rate-distortion of multi-spectral data even if the spectral channels are compressed individually. Figure 4.1 shows an overview on the proposed architecture for multi-spectral compression. As a basis for further considerations the following requirements for the entire architecture are defined:

- The compression algorithm must be able to compress images in lossless as well as in lossy or near-lossless mode.

- The compression algorithm must be able to process images with a dynamic range of up to $16 \mathrm{bpp}$.

- The architecture must be able to process an input data rate of $200 \mathrm{Mpx} / \mathrm{s}$ on a single spectral band ${ }^{2}$.

- The architecture must be able to compress images with arbitrary width and height.

- The architecture must be able to compress decorrelated MS image data.

- A ROI mask should be used in order to apply region-specific compression parameters. The ROI mask is either commanded by the ground station or determined by onboard image processing.

- The architecture must be able to produce a bit-stream, which can be re-assembled on request (by the ground station) in order to deliver compressed images with an application-specific spatial or spectral resolution (initial transmission). It should be possible to request more spectral, spatial or radiometric details on a certain region without re-transmitting already transferred data (update transmission).

- The architecture must be usable for space application, i. e. all components must be space qualified. Space qualified means products which are designed and tested

${ }^{2}$ This roughly corresponds to the pixel rate of the PAN half module of KOMPSat-3A, which has a pixel rate of $174 \mathrm{Mpx} / \mathrm{s}$. 
to meet the special electrical, mechanical or environmental requirements on-board spacecraft. The components must have a high reliability, a low power consumption, a high operating temperature range and they must be shock resistance and able to operate in vacuum, under radiation. These properties are ensured either by design or by testing.

- The architecture should be implemented on a FPGA, so that further modules, i. e. for the detector interface, can be integrated in the final design.

- Since a system based on the architecture is to be used in future mission, it must also be scalable to higher input data rates.

\subsection{Quantitative Requirements Analysis}

The CCSDS 122.0-B-1 algorithm supports lossy as well as lossless compression mode. For lossless compression, transforms for the spectral and spatial decorrelation must be reversible, and must not use quantization. The Floating Point (FP) DWT is not reversible and cannot be used for lossless compression. In the case of lossy or near-lossless compression, the reversible "Integer DWT" is not advisable, since it produces significantly higher distortions than the FP DWT [25]. On the other hand, the Integer DWT has a significant lower complexity and it is to be expected that it needs significant less resources and that it can be operated much faster in the FPGA. In order to determine the real resource consumption and maximum achievable clock speeds, both transforms will be implemented.

Although it depends on the FPGA and design optimizations, a maximum clock frequency of $100 \mathrm{MHz}$ can be assumed for a synchronous FPGA design on a recent FPGA (e. g. Virtex-5QV [128] or Microsemi RTG4 [81]). To achieve a compression rate of $200 \mathrm{Mpx} / \mathrm{s}$, in average two pixels must be processed in one clock cycle. A dynamic range of up to 16 bit for the input image data is necessary for remote sensing image so that the input data rate will be up to $3200 \mathrm{Mbit} / \mathrm{s}$. Since the compression efficiency depends on the entropy of the image, it may happen that the output data rate is nearly equal to the input data rate. In fact, the output data rate can be even higher: The compression of images with equally distributed random values and a dynamic range of 8 bit gives a compression ratio of approximately $1: 1.1$.

Image data compression at these high data rates can only be achieved by parallelization, pipelining, and stream-processing. Since an FPGA has limited internal memory, the memory requirements of each component must be estimated and optimized. Since the number of lines of an image acquired by a line detector depends on the line-rate and the measurement time and is in principle unlimited, the complete image cannot be stored most likely without external memory. A fundamental principle of the presented architecture is, that storing of a complete image before compression is not performed. The reason for this approach is that storing images would require large memory for the compression to be efficient. A line-based architecture (see Section 2.3.1.2) will be used 
for the two-dimensional DWT, because it requires the least amount of memory. After wavelet transform, the coefficients will be grouped in blocks and segments. Thus, the entropy coding process can start while image acquisition. While the width of the image decisively determines the amount of memory needed for the two-dimensional DWT, the height of the image has no influence on it, since only some of the previous lines must be stored in memory (see following sections).

ROI coding should be a feature of the new compression architecture. An investigation on the possibilities of ROI coding was performed in Section 3.1. ROI will be realized by controlling the compression parameters of the segments, i. e. a segment corresponds either (partly) to an image region inside a ROI or not. The concept of scalability was presented in Section 3.2. It should be part of the architecture concept.

The Hardware Operating System presented in [64] is used to achieve a high degree of platform independency. Since recent DLR projects such as KOMPSat-3A already use Xilinx FPGAs, the radiation hardened Virtex 5QV FPGA is chosen as the target design platform. However, this FPGA and the corresponding design tools are not available for this thesis due to U.S. export restrictions. Thus, investigations on resource consumption, power consumption, and data throughput are performed on a different FPGA: The commercial Virtex 5 XC5VFX130T is a similar FPGA in terms of resources. The power consumption of a Xilinx FPGA can be reliably estimated by the Xilinx Power Estimator (XPE) [130]. The maximum clock frequency of the Virtex 5QV is slightly lower than the maximum clock frequency of the commercial XC5VFX130T. Since an accurate estimation of the maximum clock frequency can only be performed with the export regulated synthesis tools, the maximum frequency of the Virtex 5QV is estimated to be $80 \%$ of the maximum frequency of the commercial Virtex 5 XC5VFX130T.

\subsection{Structure of the proposed architecture}

In this section, the design of an architecture fulfilling all the requirements will be derived. In order to fulfill the high demands on the data throughput and still achieve an effective and flexible compression architecture, a pipeline stream-processing approach is chosen, i. e. all steps of the compression algorithm are executed as early as possible and in parallel in order to minimize the memory required to buffer intermediate results (task parallelism). Furthermore, the Pipe communication approach presented in Section 2.3.3.3 will be used. It allows to back-propagate a busy signal from sink to source. Concatenating Pipes leads to stream processing. The design is optimized by double-buffering, adjusted buffer sizes, and pipeline stages, so that the busy signal is never raised at the input Pipe due to internal events. To keep the complexity of a superordinate system as low as possible, it is desirable to maximize the data throughput of a single compression core. If the performance of a single compression core is still not adequate, several of these cores have to be used (data parallelism). 


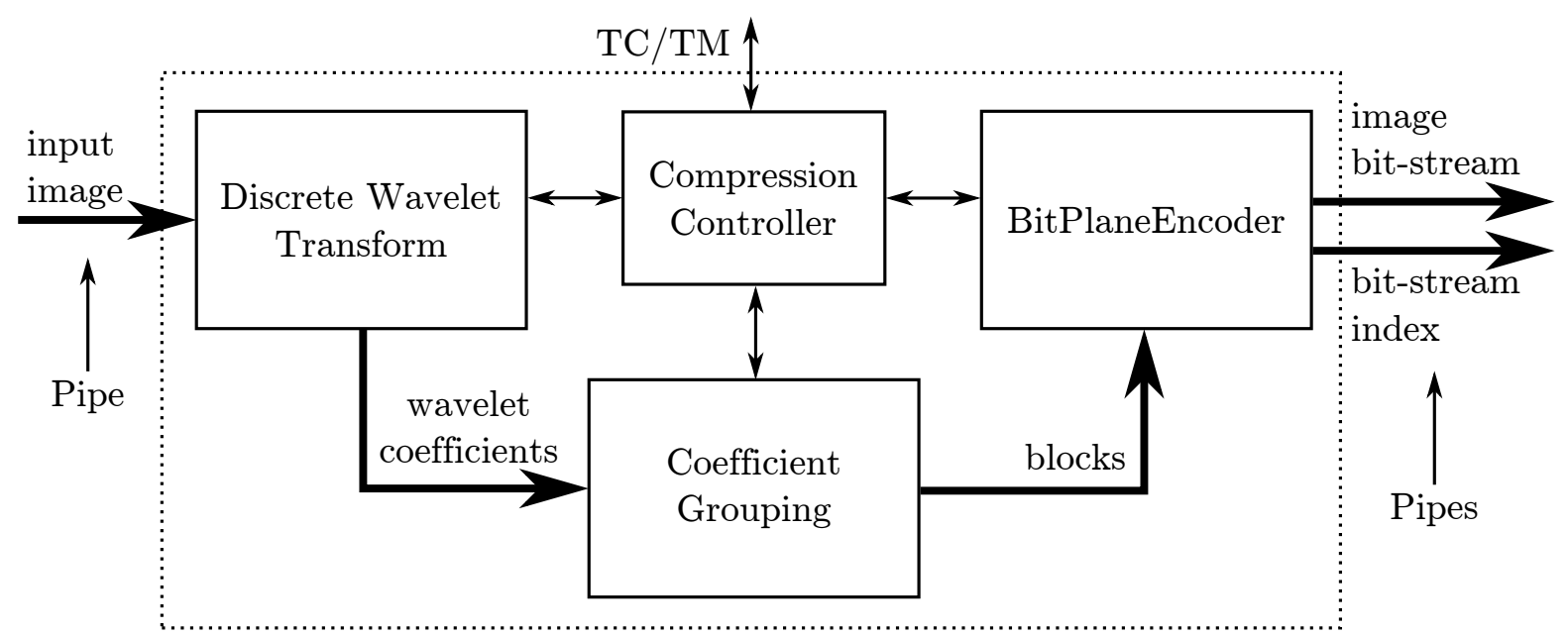

Figure 4.2: Structure of the proposed architecture.

Figure 4.1 shows the underlying structure of the proposed architecture. The design of the compression architecture consists of five main modules:

- Compression Controller Module (CCM)

- Wavelet Transform Module (WTM)

- Coefficient Grouping Module (CGM)

- BitPlaneEncoder Module (BPEM)

This structure was derived from the basic structure of the baseline CCSDS 122.0-B-1 encoder consisting of a wavelet transform module and the so-called BitPlaneEncoder (see Figure 2.24). The CCM communicates with the spacecraft via TC and Telemetry (TM) and controls all modules of the architecture. Compression parameters and a ROI mask is also supplied by the CCM. The data path starts at the WTM. The WTM applies a three-level two-dimensional wavelet transform on the images. The CGM is used to re-arrange the coefficients from the ten sub-bands and to form the blocks and segments necessary for the BPEM. A memory controller for external memory such as static random-access memory (SRAM) is not shown in the architectural overview, but will be used if necessary (see Section 4.3.5).

For image data, the architecture has an input Pipe consisting of two 16-bit input vectors including a data valid signal. It processes two image pixels per clock cycle. This choice was made because it allows to increase the data throughput without increasing the maximum clock frequency. Furthermore, the wavelet transform modules produce their outputs every two input values. This is not a practical limitation, since a detector can operate in a different (faster) clock domain. Furthermore, two or more readouts of a detector can be combined into a single data stream using on-chip FPGA memory. It is common to perform some pre-precessing before image compressing so that the data stream can be 
adapted to the compression core. Current instruments developed at DLR have integrated flat field or radiometric correction. The output bit-stream uses a 64-bit interface with an 8-bit wide valid signal that indicates which of the 8 bytes of the output word are valid.

In the following sections, a design of each individual module is presented. The following design goals were pursued:

1. Maximize data throughput: A maximum data throughput is to be achieved.

2. Minimize the amount of external memory: External memory needs additional printed circuit board (PCB) area, power, and FPGA resources. Ideally, the architecture shall not rely on external memory.

3. Optimize the FPGA's internal resource consumption.

\subsubsection{Compression Controller}

The task of the compression controller is to manage the compression parameters via TC and TM, and to provide them to the requesting modules. The current design has only a rudimentary interface and has to be adapted to project-specific requirements. The following parameters can be set during operation (implemented as VHDL port parameters). The individual parameters correspond to parameters of the CCSDS 122.0-B-1 standard, except ImageWidth and ImageHeight which correspond to the actual size of the input image.

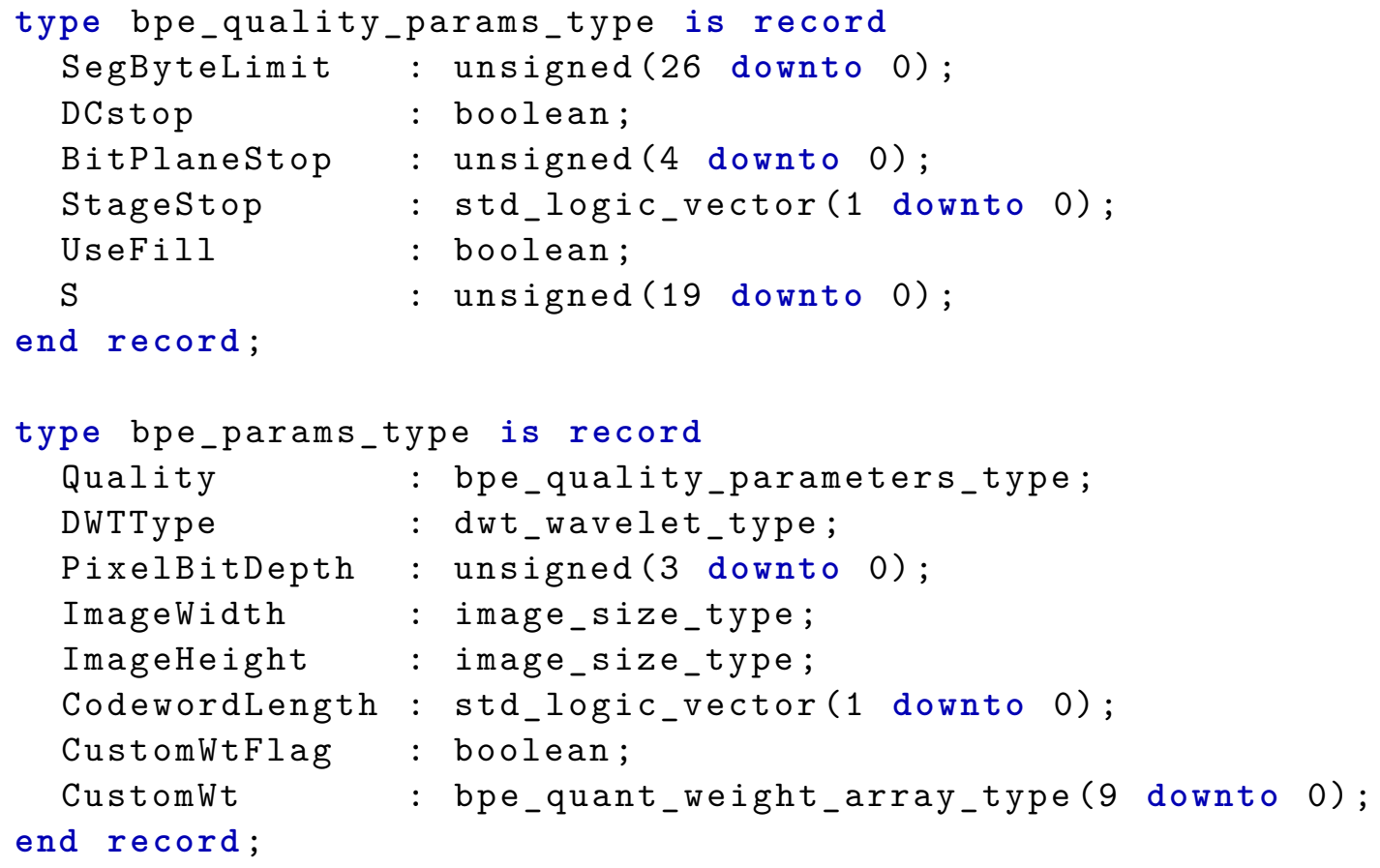


In an adapted implementation, all these parameters can be set constant before VHDL synthesization. In case of parameters such as DCstop, BitPlaneStop, or StageStop, the complexity of the resulting architecture could significantly be reduced resulting in higher clock maximum frequencies and lower resource consumption. The architecture uses an internal parameter structure initilized by the CCM to pass and update values that are calculated based on statistics of the current segment such as the bit-depth of the DC coefficient or AC coefficients:

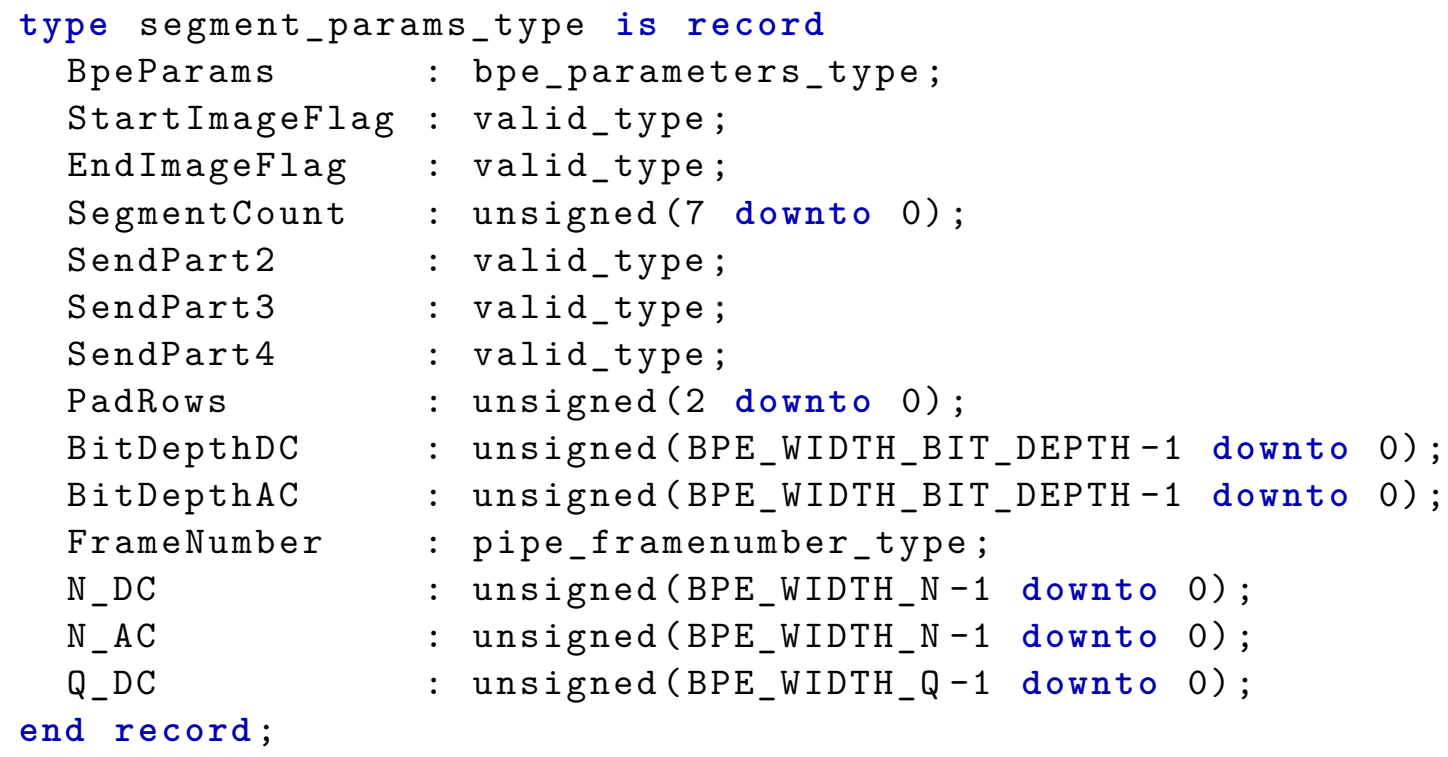

\subsubsection{Wavelet Transform Module}

The overall structure of the top-level WTM is shown in Figure 4.3. Three 2D-DWT modules each with its own memory are concatenated. A line-based architecture (see Section 2.3.1.2) was chosen for the three individual 2D-DWT modules in order to achieve maximum data throughput. A direct-scan architecture cannot be used, because the input images are very large or basically not limited in size. It is not possible to store an whole transformed image in a temporary memory. Since a maximum data throughput is the primary design goal, folding approaches to reduce the resource consumption and to increase the utilization are not intended to be used. The modules use Pipe communication for input and output data. If the FP DWT is used, the input and output data is converted between integer and floating-point format. The floating-point support is optional during VHDL synthesization, i. e. the functionality may completely be removed. The following parameters are provided via separate signals:

- Current image size (width and height)

- Type of wavelet transform (Integer or FP DWT) 


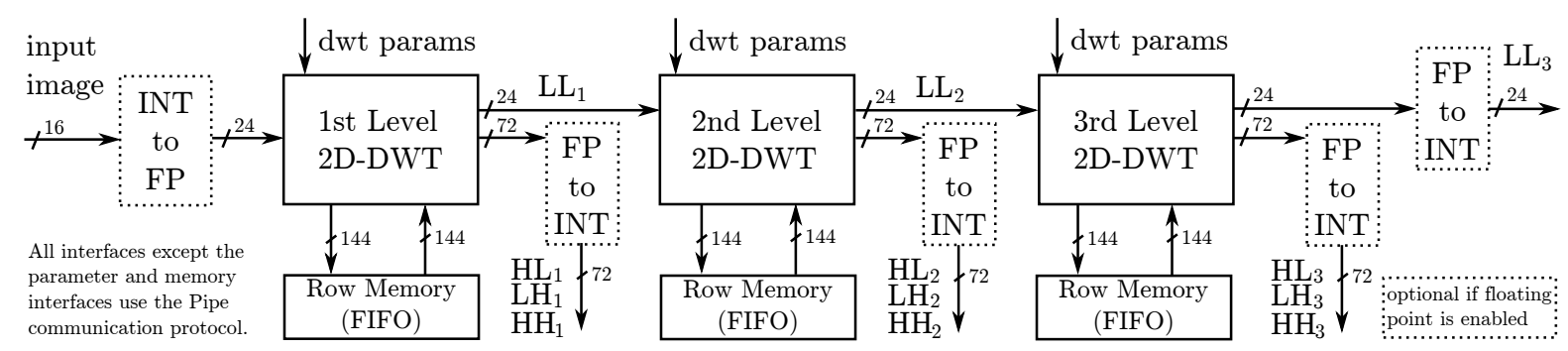

Figure 4.3: Structure of the top-level WTM.

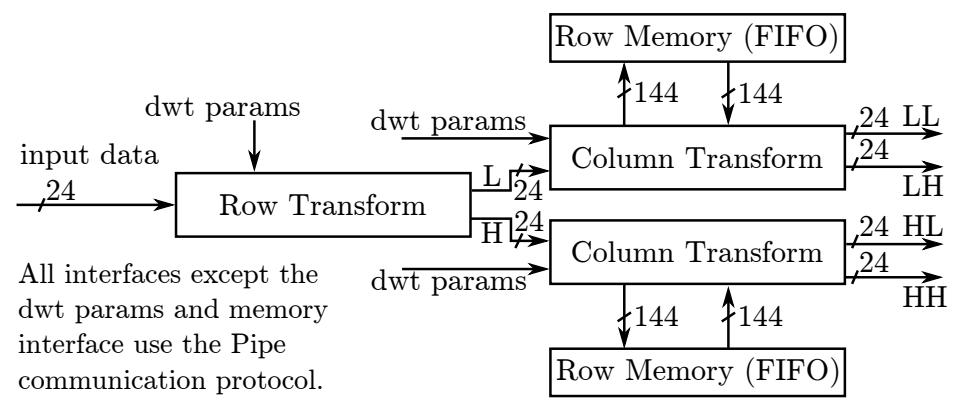

Figure 4.4: Structure of a single 2D-DWT module. A row transform module that produces a low-pass (L) and a high-pass (H) image which where then separately transformed by column transform modules.

The memory can either be external memory (e.g. SRAM) or FPGA-internal memory (slice registers for some coefficients or BlockRAM for image lines). In order to reduce the design effort, a concept for using external memory is presented in Section 4.3.5.

Figure 4.4 shows the structure of a 2D-DWT module. The row and column transform are usually separable, i. e. they can be executed in any order. However, the Integer DWT, recommended in the CCSDS 122.0-B-1 standard, leads to different results depending on the execution order. In order to maintain compatibility, column transform after row transform is chosen. For the row transform, only some intermediate results or wavelet coefficients must be buffered. Thus, slice registers are used and no larger memory such as BlockRAM or external memory is needed. For the column transform, several lines of image pixels or wavelet coefficients must be buffered (see below). The structure of the row and column transform modules is shown in Figure 4.5 and Figure 4.6.

\subsubsection{Row and Column DWT Module}

The row transform module uses a single input Pipe for input data and two output Pipes for the high- and low-pass coefficients. A finite state machine (FSM) controls the communication on these three Pipes and the DWT pipelines. The sub-band width and height as well as the type of the DWT are separately passed as parameters. Depending on the DWT type, either the Integer or FP DWT (if available) is used. Both pipelines use internal buffers for pixel mirroring at the image boundaries. The FSM feeds the 


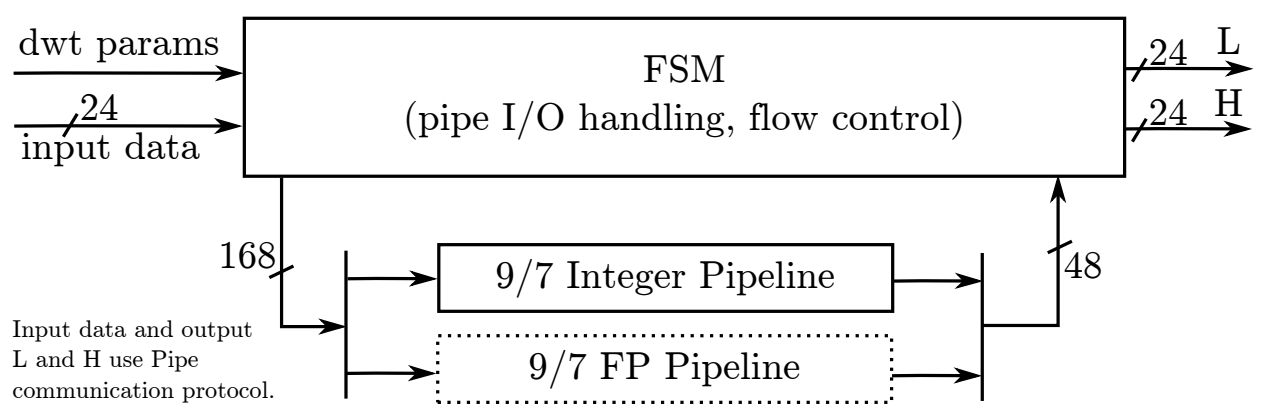

Figure 4.5: Overview of the structure of the row DWT.

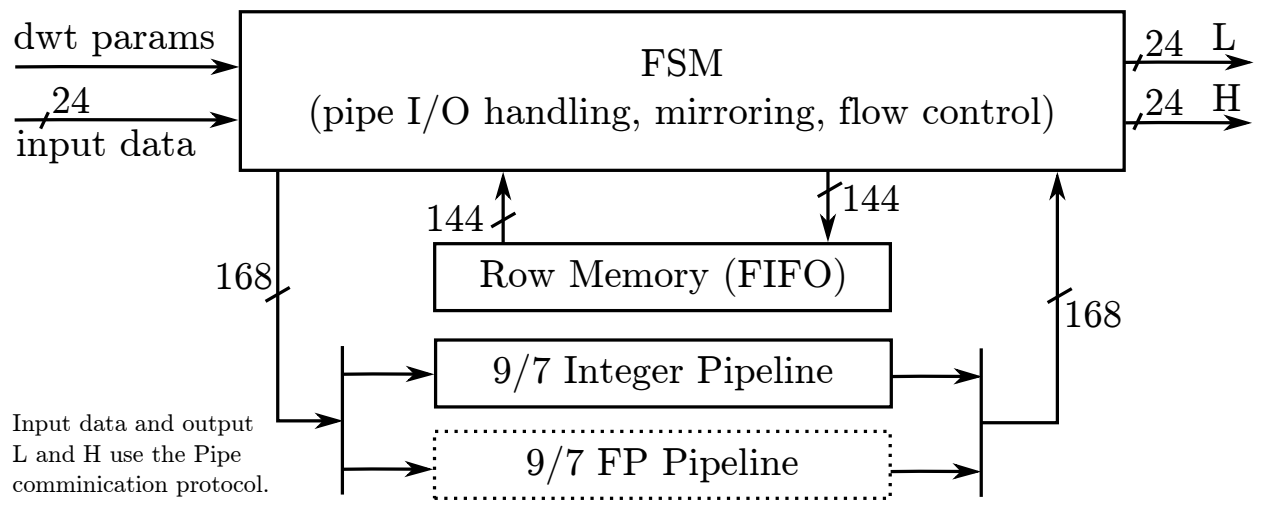

Figure 4.6: Overview of the structure of the column DWT.

corresponding DWT pipeline with input data (6 values from memory and current input) and the current image or data position (for mirroring) and forwards the output of the pipeline to the low- and high-pass output Pipe channels.

The column transform module operates according to a similar principle. The only difference is that the control FSM performs the mirroring and that a separate memory is used to buffer lines of input words or coefficients. The 6 values are read from memory and passed together with the current input to the pipelines. After the pipeline calculation has been finished, the 6 values stored in memory are updated for the next column transform step and 2 values are issued.

\subsubsection{Data Rates and Memory Demands}

The input data transfer rate $d_{1}$ of the $1^{\text {st }}$ level module corresponds with the input data rate $d_{i n}$ of the compression architecture:

$$
d_{1}=d_{\text {in }}=2 \mathrm{px} \cdot 100 \mathrm{MHz}=200 \mathrm{Mpx} / \mathrm{s}
$$

The data transfer rate of the $2^{\text {nd }}$ level module is as follows:

$$
d_{2}=1 / 4 \cdot d_{1}=50 \mathrm{Mpx} / \mathrm{s}
$$


The data transfer rate of the $3^{\text {rd }}$ level module is as follows:

$$
d_{3}=1 / 4 \cdot d_{2}=12.5 \mathrm{Mpx} / \mathrm{s}
$$

Since the $2^{\text {nd }}$ and $3^{\text {rd }}$ level module has a lower utilization, it would be possible to lower the clock frequency or to share resources between these modules. However, this has not been implemented. Additional clock domains would require additional synchronization logic and in order to benefit from a slower clock frequency, the pipeline stages must be adapted. If the (combinatorial) resources would be shared between the modules (using a time multiplexing scheme), additional de-/multiplexing control logic would be necessary. In both cases, the approach has not been investigated and does not seem promising with regard to possible resource savings.

The memory demands of the row transform modules are negligible, since only some input values must be stored in memory. The memory demands of the 2D-DWT are dominated by the column transform, and are dominated by the image width (number of columns) and the chosen wavelet (Integer or FP). The number of "lines" is higher for the Integer DWT, since there is no two-stage lifting scheme for this. For each wavelet decomposition level, effectively 6 lines must be stored or buffered for the column transform: The Integer DWT needs 5 and the FP DWT only 4 lines to be buffered (see above). The Integer and the FP DWT pipelines share the same memory. One additional line must be buffered, since the pipelines need 2 input values at the same time. The total amount of memory needed for a single-level row transform is as follows:

$$
\text { memory demand }(l)=\frac{1}{2^{l-1}} \cdot I_{w} \cdot R_{\max } \cdot 24 \text { bit }
$$

where $I_{w}$ denotes the image width, $l$ denotes the level of the dyadic decomposition and $R_{\max }=6$ denotes the maximum number of lines, which must be stored in memory. For a three-level dyadic decomposition, the total amount of memory is as follows:

$$
\text { total memory demand }=\sum_{i=1}^{3} \operatorname{memory} \operatorname{demand}(l)=\frac{7}{4} \cdot I_{w} \cdot R_{\max } \cdot 24 \mathrm{bit}
$$

For $R_{\max }=6$ and an image width of $I_{w}=1024 \mathrm{px}$, the total buffer size is $10752 \mathrm{px}$ (or 31.5 kbyte for $d=24 \mathrm{bpp})$. For an image width of $I_{w}=16384 \mathrm{px}$, the total buffer size is $172032 \mathrm{px}$ (or $504 \mathrm{kbyte}$ for $d=24 \mathrm{bpp}$ ).

As already noted, up to six lines must be stored in memory, which means that up to six temporary values must be read and written every clock cycle. Assuming a data width of $d=24$ bit and a clock frequency of $100 \mathrm{MHz}$, the total data rate (read and write) of the memory is as follows:

$$
\text { total memory data rate }=2 \cdot R_{\max } \cdot 24 \mathrm{bit} \cdot 100 \mathrm{MHz}=3600 \mathrm{Mbyte} / \mathrm{s}
$$

It should be noted that this value is independent of the image size. 


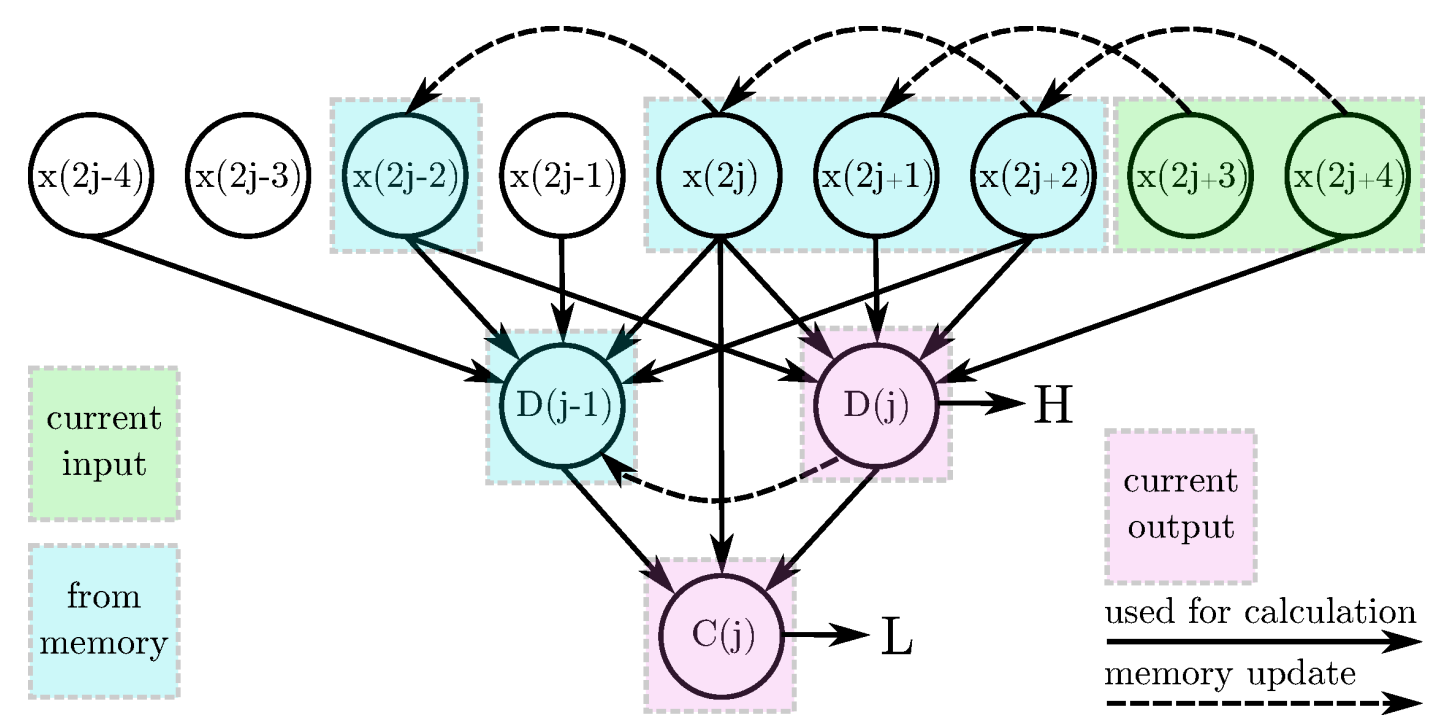

Figure 4.7: Structure of the Integer DWT pipeline.

\subsubsection{Arithmetic Pipelines}

Figure 4.7 shows the structure of the Integer DWT pipeline. If input values for two lines are to be processed, the corresponding entries stored in memory are read, both together is processed in the pipeline, and updated memory values are written. The pipeline implements a principle similar to the lifting scheme: Intermediate results of the wavelet transform are stored to memory and are used for the next iteration. If input values of two pixels (row transform) or lines (column transform) are present at the input, the corresponding intermediate results stored in memory are read. Both together are processed in the pipeline and the resulting wavelet coefficients are issued at the output. The updated intermediate results are written to memory. The Integer DWT pipeline structure is used for the row as well as the column transform. Since the row transform processes the input sequentially, it uses memory based on slice registers. The row transform uses memory based on BlockRAM, because it processes all input rows in parallel and thus needs to store the intermediate results for each of the input columns. The column Integer DWT pipeline needs to buffer 5 values (4 input values and 1 intermediate result) for each input column. In both variants, three pipeline stages are inserted in order to achieve the desired target frequency of $100 \mathrm{MHz}$. Equations (2.49) and (2.50) are used to generate the current output. At the end, the memory is updated for the next iteration.

In case of the FP DWT, the lifting scheme can be implemented directly. The principle is the same as for the Integer DWT pipeline. Again, input values of two pixels or lines must be present at the input. The corresponding intermediate results of the lifting scheme are read from memory. The calculation itself is performed in a 13-stage pipeline (2 adders and 1 multiplier for each of the 4 lifting stages, 1 stage for the final output multiplication). 


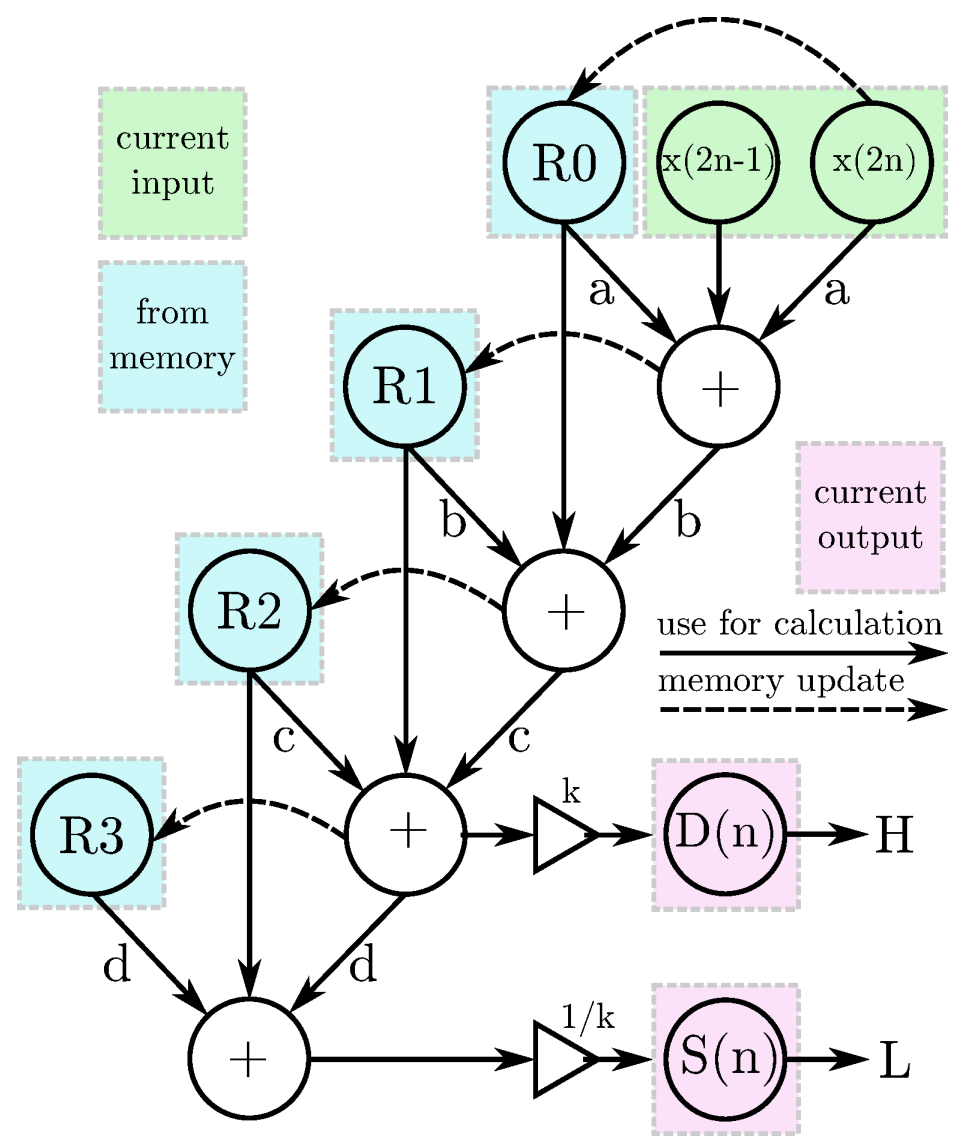

Figure 4.8: Structure of the FP DWT pipeline.

The column FP DWT pipeline needs to buffer 4 values (1 input value and 3 intermediate results) for each input column. Figure 4.8 shows the lifting structure.

\subsubsection{Parallel Border-Less DWT}

As already mentioned, wavelet-based image compression algorithms perform the DWT on large areas (ideally the whole image) instead of small regions in order to obtain the maximal compression ratio and to avoid compression artifacts at the region boundaries. Thus, the partitioning or block formation must be done in the wavelet domain, i. e. after the DWT.

A similar problem will also occur, if a system based on the compression architecture is not able to process the input data in real-time: The only solution for still achieving online compression is to parallelize the compression architecture in a way that the input data stream is distributed to multiple compression cores. This leads to a tiling of the image in the spatial domain, which results in discontinuities after the decompression.

The approach used in this thesis is to avoid these compression artifacts by transferring border pixels of the image between the parallel DWT cores as illustrated in Figure 4.9. 


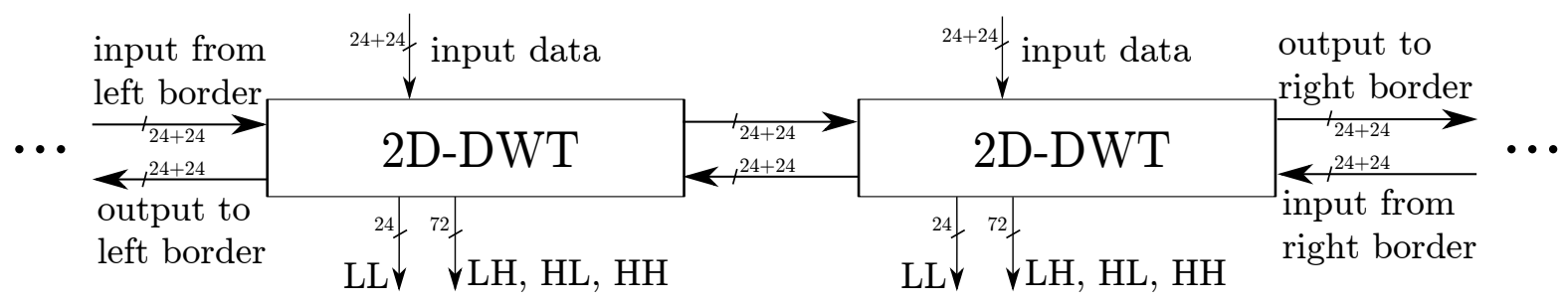

All interfaces are using the Pipe communication protocol. Handshake and flow control signals (request, confirm, data valid, and busy) are not shown.

Figure 4.9: Overview on the approach for the parallel border-less DWT.

The advantages of this solution are that there is little overhead for transferring the border pixels and that the results of multiple DWT cores are identical to the results of a single, but faster DWT core.

\subsubsection{Coefficient Grouping Module}

The CGM is a fundamental module needed to achieve the required data throughput. The BPEM reads the wavelet coefficients block by block, until the required number of blocks has been read (see Figure 2.24) and the entropy coding process can start. Unfortunately, this is not the order in which the WTM produces the wavelet coefficients, since a single image line might correspond to multiple segments and the wavelet coefficients of levels $L_{1}, L_{2}$, and $L_{3}$ have a non-negligible time delay between each other.

Even through a line-based architecture is considered to be optimal for the compression architecture to be developed, it has two problems. First, a CCSDS 122.0-B-1 encoder usually operates in stripe-based mode, i. e. the segment size is $S=S_{x}=N \cdot\left\lceil I_{w} / 8\right\rceil$ blocks, where $I_{w}$ denotes the width of the image and $N$ is an integer $\geq 1$. In order to support ROIs, the segment size must be variable or at least less than this value. In stripe-based mode, all blocks in any image line belong to the same segment. Otherwise, the WTM generates coefficients belonging to multiple segments. The second problem is that the structure of the WTM shown in Figure 4.3 causes temporal delays of the wavelet coefficients in the higher decomposition levels $L_{2}$ and $L_{3}$.

An alternative approach would be to transform only image parts needed to code the current segment. But since the segments overlap in the spacial domain, this approach would require to buffer large parts of the input image and to re-compute wavelet coefficients at the image boarders. This would significantly increase the memory requirements and decrease the resulting data throughput.

The main task of the CGM shown in Figure 4.10 is to re-arrange the wavelet coefficients to a block- and segment-wise output. It buffers the wavelet coefficients of the $L_{1}$ and $L_{2}$ decomposition levels and synchronizes its output to $L_{3}$. Consequently, it also buffers coefficients belonging to other segments. The CGM consists of four sub-modules: 


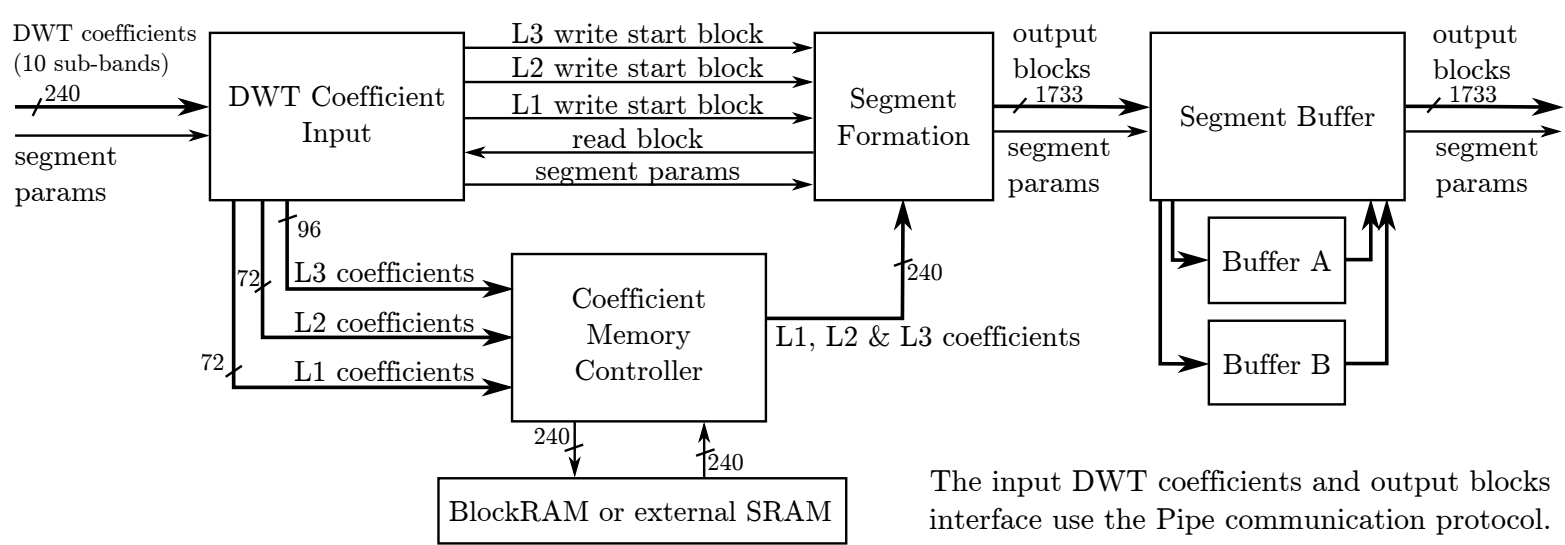

Figure 4.10: Structure of the Coefficient Grouping Module.

- DWT Coefficient Input Module

- Memory Access Scheduler and Controller Module

- Segment Formation Module

- Segment Buffer Module

\subsubsection{DWT Coefficient Input Module}

This module receives the DWT coefficients of the WTM (three 2D-DWT modules producing 10 sub-bands of wavelet coefficients) and writes them to temporary memory. Therefore, it has to determine the block number a coefficient belongs to. The DWT Coefficient Input Module and the Segment Formation Module share a circular list of memory block addresses. The DWT Coefficient Input Module signals to the Segment Formation Module, the position of the first $L L_{1}, L L_{2}$, and $L L_{3}$ blocks that have not been completely written. In turn, the Segment Formation Module signals the position of the last block that has been completely read. Furthermore, segment parameters are requested from the compression controller. Parameters such as StartImageFlag and EndImageFlag, which indicate the first and last segment, are updated and sent to the Segment Formation Module.

\subsubsection{Coefficient Memory Controller Module}

The Coefficient Memory Controller Module provides an abstract interface to either FPGA BlockRAM or external memory. In the case of FPGA BlockRAM, the implementation is pretty simple, since the BlockRAM has just to be instantiated. In case of external memory such as SRAM, a memory controller has to be used and - if multiple design elements use the same memory device - the memory has to be shared. For design simplification, the memory concept presented in Section 4.3.5 is used.

The size of the memory buffer depends on the number of blocks that must be buffered. This in turn depends on the length of the wavelet filter and the width of the image. At 
the low-pass filter of the FP DWT, eight input values must be available around the current input value ( 4 before and 4 after), which results in the low-pass filter length of $4+1+4=9$. With a line-based architecture, this will cause an output latency of four rows for each decomposition stage. In other words this means that the $L L_{k+1}$ wavelet coefficients depend on the corresponding input pixel position in $L L_{k}$ with a radius of four pixels or an image area of up to $9 \cdot 9$ pixels.

Every 2D-DWT module $L_{k}$ decomposes an input signal $L L_{k}$ into the four sub-bands $L L_{k+1}, H L_{k+1}, L H_{k+1}$, and $H H_{k+1}$. The first output will arrive a few clock cycles after the fifth input line has started. From now on, the 2D-DWT module outputs wavelet coefficient every second line (due to the sub-sampling). When the first output arrives in the 2D-DWT module $L_{3}$, the 2D-DWT module $L_{1}$ has already emitted 12 lines of wavelet coefficients. At this time, 2D-DWT module $L_{2}$ has emitted 4 lines of wavelet coefficients. Thus, at least $3 \cdot S_{x}$ blocks must be buffered for synchronization with the $L_{3}$ sub-band, where $S_{x}$ denotes the maximum number of blocks in horizontal direction.

A value of $4 \cdot S_{x}$ is chosen for our design in order to buffer more blocks. Thus, assuming a dynamic range of the wavelet coefficients of $d=24$ bit, the total amount of memory necessary for coefficient grouping is as follows:

$$
4 \cdot S_{x} \cdot 64 \text { coefficients } / \text { block } \cdot 24 \text { bit }=S_{x} \cdot 0.75 \text { kbyte }
$$

If the image has up to $S_{x}=2048$ blocks in horizontal direction, the CGM requires 1.5 Mbyte of memory. An external memory is necessary to buffer and re-arrange the DWT coefficients (see Section 4.3.5).

Since all the coefficients need to be written and read only once, the data rate of the memory is twice the input data rate of the module. The input data rate of the module is $200 \mathrm{Mpx} / \mathrm{s}$. Thus, the required memory bandwidth (read and write) is $400 \mathrm{Mpx} / \mathrm{s}$. Assuming a dynamic range of the wavelet coefficients of $d=24$ bit, the total memory bandwidth requirement is $1200 \mathrm{Mbyte} / \mathrm{s}$.

\subsubsection{Segment Formation Module}

If the Segment Formation Module has received the segment parameters from the DWT Coefficient Input Module, it transfers all blocks that were completely written to memory, to the Segment Buffer Module, until the desired number of blocks for the segment has been read (segment size $S$ ). While reading the blocks from memory, the module converts the AC coefficients to sign/magnitude-representation and determines BitDepthDC and BitDepthAC for the segment and BitDepthAC_block for each block.

The block data consists of the DC coefficient (27 bits), $63 \mathrm{AC}$ coefficients $(63 \cdot 27 \mathrm{bits}=$ 1701 bits), and the bit-depth of the $\mathrm{AC}$ coefficients ( 5 bits): 


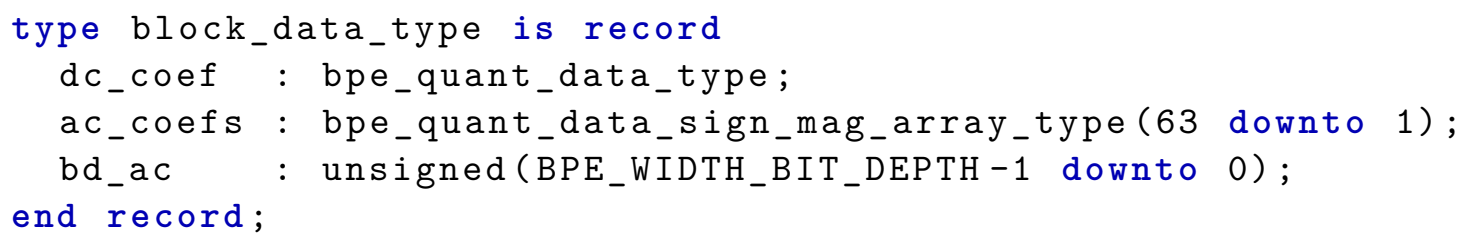

Again, the segment parameters are updated and sent to the Segment Buffer Module.

\subsubsection{Segment Buffer Module}

The Segment Buffer Module provides blocks to the BPEM. A double buffering mechanism is used, so that the next segment can be written to the buffer, while the BPEM processes the current segment. The module buffers the weighted coefficients in sign and magnitude representation, each consisting of $d+4$ bit, as well as the bitdepth of the AC coefficients of each block ( $5 \mathrm{bit}$ ). If the dynamic range of the coefficients is $d=24$ bit, the total size of the buffer is

$$
2 \cdot 64 \cdot S \cdot 28 \text { bit }+5 \text { bit }=S \cdot 448 \text { byte }+5 \text { bit }
$$

Assuming a segment size of $S=128$ blocks, the memory size will be $\approx 56$ kbyte. The FPGA internal BlockRAM should be sufficient (see also Table 4.1).

\subsubsection{BitPlaneEncoder Module}

The BPEM implements the entropy encoder of the compression algorithm and produces or assembles the individual bit-stream parts. It gets the input from the CGM. Without considering parallel execution mechanisms, it must be able to compress each segment in $32 \cdot S$ clock cycles. In order to achieve real-time compression, the individual modules must operate in parallel.

The structure of the proposed BitPlaneEncoder Module is shown in Figure 4.11. The BPE Control Module reads the segment blocks from the CGM (more specifically the Segment Buffer Module). Parameters necessary to write the segment header are also provided, so that the coding process can start immediately. Segment header, quantized DC coefficients, additional DC bit-planes, and AC coefficient bit-depths are processed sequentially, while the stages $0-4$ are processed in parallel. The reason for this is that stage coding produces the major part of the compressed bit-stream and consumes the majority of the execution time (see Section 3.2.1.1). As in the WTM, all sub-modules use the Pipe communication presented in Section 2.3.3.3. All coding modules use a data valid mask (one bit for each data bit) in order to mark valid output bits, and two flags in order to mark the end of a segment or a bit-plane (end-of-segment and end-of-bit-plane).

\subsubsection{BPE Control Module}

The BPE Control Module is shown in Figure 4.12 controls the coding modules and feeds their inputs with the corresponding input data. It does not wait until a coding module 


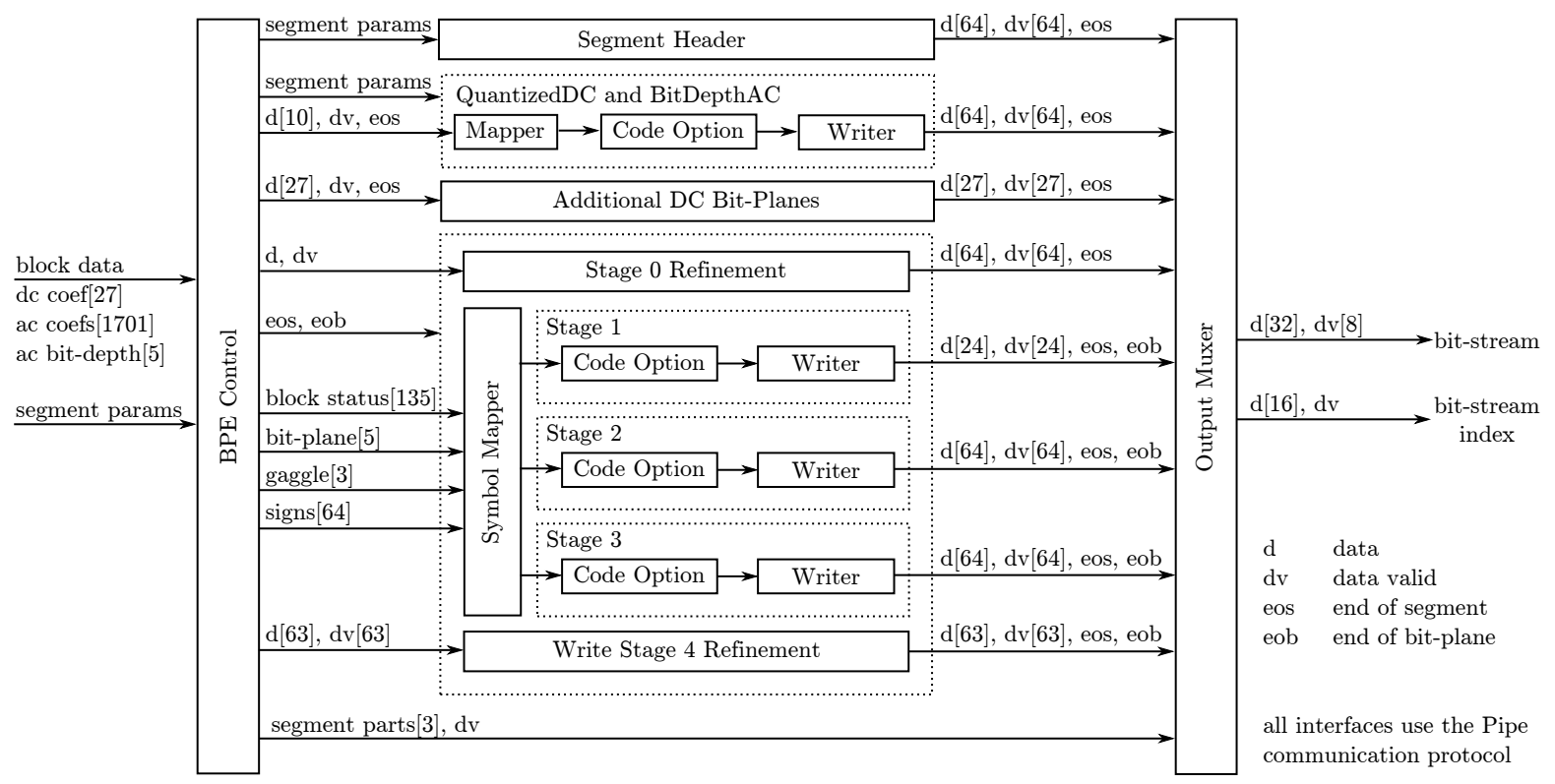

Figure 4.11: Overview on the proposed BitPlaneEncoder Module.

has finished processing, i. e. all coding modules operate in parallel. If the module has sent all information to the subsequent coding modules required to code a segment, it immediately starts with the next segment. The information about which parts belong to the current output segment is sent to the Output Muxer Module.

Whenever the segment buffer has collected an entire segment, it sends a request (input req) to the BPE Control Module. When the compression of the segment is finished, the module confirms this to the segment buffer (input ack). During operation, the BPE Control Module sequentially generates input data for the coding modules.

After receiving input req from the Segment Buffer Module, the Pipe communication to all subsequent modules (Segment Header, QuantizedDC and BitDepthAC, ..., see Figure 4.12) is initialized. Before sending data or requests to each of the modules, the module signals the Output Muxer about bit-stream parts to be expected. Then, the module send the segment parameters to the Segment Header Module and signals the module to start writing the segment header. It does not wait for the segment header being written to the Output Muxer or output bit-stream and immediately switches to the next step of writing the DC coefficient of each block to the QuantizedDC and BitDepthAC Module. The DC coefficients are read from memory (one per clock cycle, $S$ clock cycles) and passed to the module. Depending on the compression parameters, the BPE Control Module stops coding, goes to the next step of coding the additional DC bit-planes, or goes to the step of coding the bit-depth of the AC coefficients.

For the additional DC bit-planes, the BPE Control Module re-reads the DC coefficient of each block of the segment (one per clock cycle, $S$ clock cycles). At this point the 


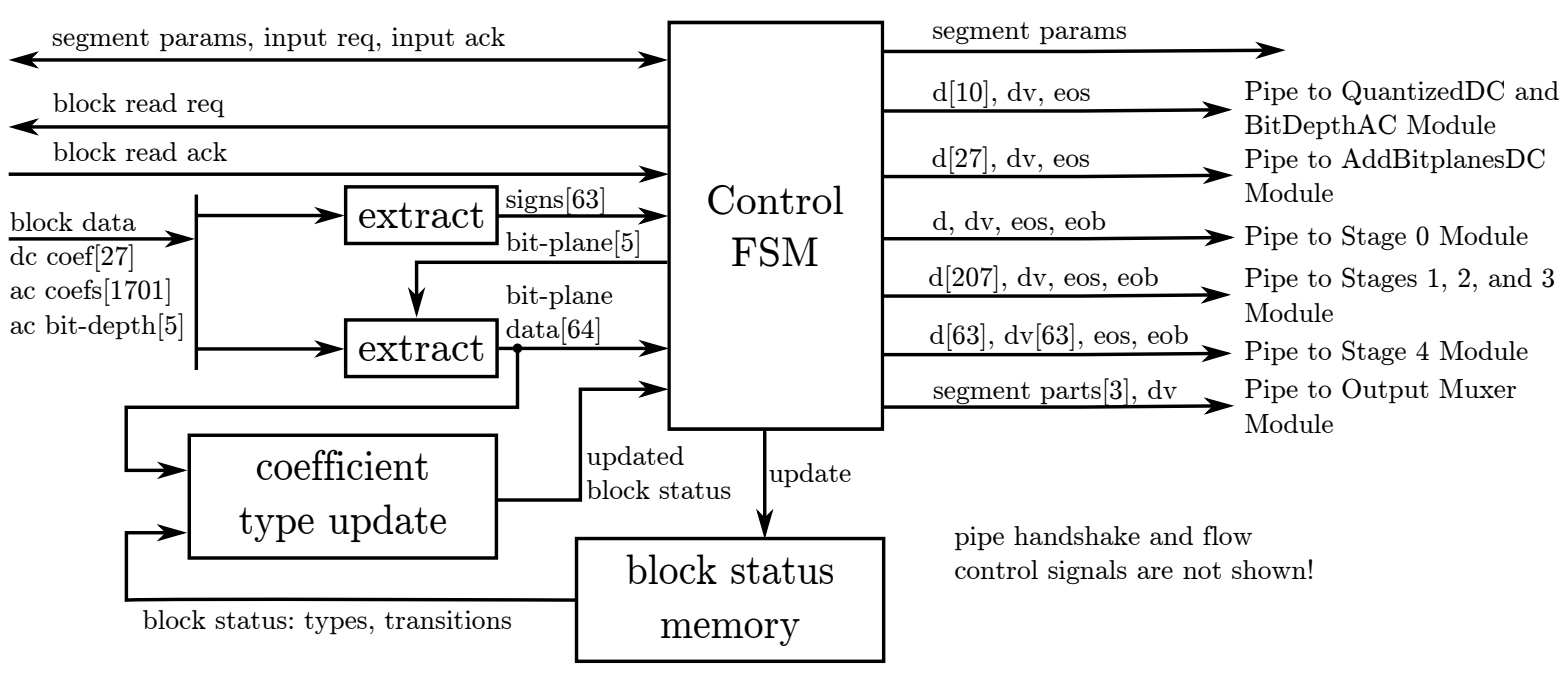

Figure 4.12: Overview on the BPE Control Module.

implementation differs from the standard: In the CCSDS 122.0-B-1 standard, the ordering of the bits is (bit-plane, bit-position) meaning that all bits from a certain bit-plane are transferred before sending the bits of the next bit-plane. This was swapped in this implementation, because it will reduce the number of read accesses to the segment buffer memory. The total number of bits will be the same and thus, the compression efficiency is not influenced by this procedure. Depending on the compression parameters, the BPE Control Module stops coding, or goes to the next step of coding the additional DC bit-planes, goes to the step of coding the bit-depth of the AC coefficients.

The bit-depth of the AC coefficients are then encoded by re-using the QuantizedDC and BitDepthAC Module. Now, the bit-depth of the AC coefficients of each block are read from the Segment Buffer Module (one per clock cycle, $S$ clock cycles). After the AC coefficient are coded, the BPE Control Module stops coding, or goes to the next step of coding the coefficient bit-planes.

The modules coding the stages 0 to 4 are used in parallel. Two nested loops are used to feed the this modules with data: The bit-plane loop (outer) and the block loop (inner). The bit-plane loop start at BitDepthAC (see Section 2.4) and decreases until 0 or stops depending on the compression parameters. For each bit-plane, 64 bits (one bit per coefficient) of each block is read from memory. These two loops take BitDepthAC $\cdot S$ clock cycles.

\subsubsection{Segment Header Module}

At first, the segment header is transferred to the Segment Header Module. The Segment Header Module creates the segment header depending on the current segment parameters and sends it to the output muxer. 


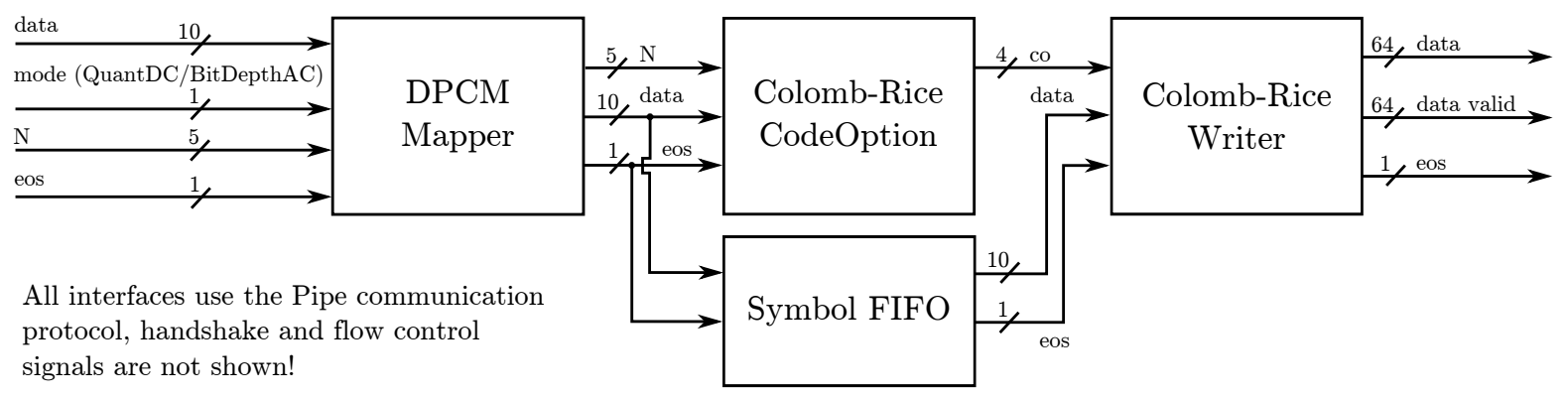

Figure 4.13: Overview on the DPCM Module.

\subsubsection{QuantizedDC and BitDepthAC Module}

Subsequently, the DC coefficient of each block is read from the segment buffer, quantized, and transmitted to the QuantizedDC and BitDepthAC Module. The module is shown in Figure 4.13. It is also used to encode the bitdepth of the AC coefficients of each block. In both cases, one block is read from the segment buffer every clock cycle. The End Of Segment (EOS) signal is internally used and propagated through the module, because it will be used by the Output Muxer Module for segment separation.

All input values are first mapped to symbols with the DPCM method presented in Section 2.4.4.3. A mode signal is indicating whether quantized DC coefficients (QuantizedDC) or the bit-depth of the AC coefficients (BitDepthAC) are presented at the input, since the mapping methods differ slightly. Then, the optimal code option $p$ for subsequent Rice coding is determined. The architecture does not support the heuristic method presented in the CCSDS 122.0-B-1 standard, since it is not a significant simplification of the algorithm. Furthermore, the effort required for the code option selection rules used by the heuristic method is similar to those required for the optimal code option determination. During the determination of $p$, the mapped symbols are stored in a small FIFO because they are also needed for final Rice coding. The depth of the FIFO should at least be equal to the maximum gaggle size $G$ and is set to $2 \cdot G$ (double buffering). Depending on the determined code option $p$, data is written uncoded in one stage or coded in two stages (see [24, p. 4-19 ff.]).

\subsubsection{Additional DC Bit-Planes Module}

In the next step, the additional DC bit planes are read from memory and transmitted to the Additional DC Bit-Planes Module. DC coefficient refinement is performed depending on the quantization factor $q$ and BitDepthAC (see Section 2.4.4.3). In this step, some bits of the DC coefficients of all blocks are encoded. The order of the encoded bits was changed: Instead of sending the $(q-1)^{\text {st }}$ most-significant bit of each DC coefficient followed by the $(q-2)^{\text {nd }}$ most-significant bit of each DC coefficient (and so on, until the BitDepthAC ${ }^{\text {th }}$ bit of each DC coefficient), bits $q-1, \ldots$, BitDepthAC of the first DC coefficient is sent, followed by the corresponding bits of the second DC coefficient and so on. This reduces the number of memory accesses to the segment buffer. 


\subsubsection{Stage Coding Modules}

After the completion of the initial coding of the segment, the encoder starts the stage coding procedure. In this step, block data is read from the segment buffer for every bit plane to be encoded. The coding process runs for the bit planes $b=$ BitDepthAC -1 , BitDepthAC $-2, \ldots, 0$. Stages $0-4$ are encoded in parallel as follows.

Stage 0 data, if valid, consists of a single bit (the corresponding bit of the DC coefficient). Stage 1, 2, and 3 data is generated from the current block status, the coding parameters, the index of the current bit-plane, the number of the current gaggle, and the signs of each AC coefficient (Figure 4.14). Stage 4 data consists of up to $63 \mathrm{bits}$, one bit for each $\mathrm{AC}$ coefficient that was selected in a previous bit-plane.

In stages 1,2 , and 3 , for every block, the types $(0,1,2$, or -1$)$ of each coefficient and the maximum types in the block hierarchy are determined. Afterward, up to 38 symbols are generated (even not all symbols can occur simultaneously). Both operations each require one clock cycle. Subsequently, the symbols are sent to the VLC Coding Modules. Similar to the QuantizedDC and BitDepthAC Module, the VLC Coding Modules consist of a Statistics Module, a FIFO buffering already mapped symbols, a CodeOption Determination Module, and a Writer Module applying the optimal code option the the symbols.

The optimal code option determination is performed in two steps: The number of bits for each symbol length and code option is summed up individually in the three Statistics Modules. After the statistics of each stage is determined, the results are fused in a CodeOption Determination Module that calculates the optimal code option for all three stages. Furthermore, the stage, in which the corresponding code option identifier must be written, is determined. The width of the data path here is 2 mapped symbols for stage 1, and 6 mapped symbols for stages 2 and 3 . The values are chosen to have a good trade-off between design complexity and parallelism. Stage 1 generates no more than 2 symbols per block. Up to 8 or 28 symbols are generated by the stages 2 and 3 respectively.

In parallel to the code option determination, the mapped symbols are buffered in FIFOs. The sizes of the FIFOs depend on the gaggle size (over which the optimal code option is determined) and the maximum number of symbols that can be generated in each stage. At the end, a VLC Writer Module encodes the mapped symbols. The output width of 64 bit is sufficient to write up to 6 mapped symbols per clock cycle: The maximum code length of a mapped symbol is 8 bit (see [24]). Since a code option identifier of 2 bit may immediately located before a corresponding code, 10 bit of the output vector are used for one coded symbol. This also explains why up to 6 symbols are sent in one clock cycle to the VLC Coding Modules.

Stage 4 data consists of up to 63 bits - one bit for each AC coefficient that was selected in a previous bit plane. 


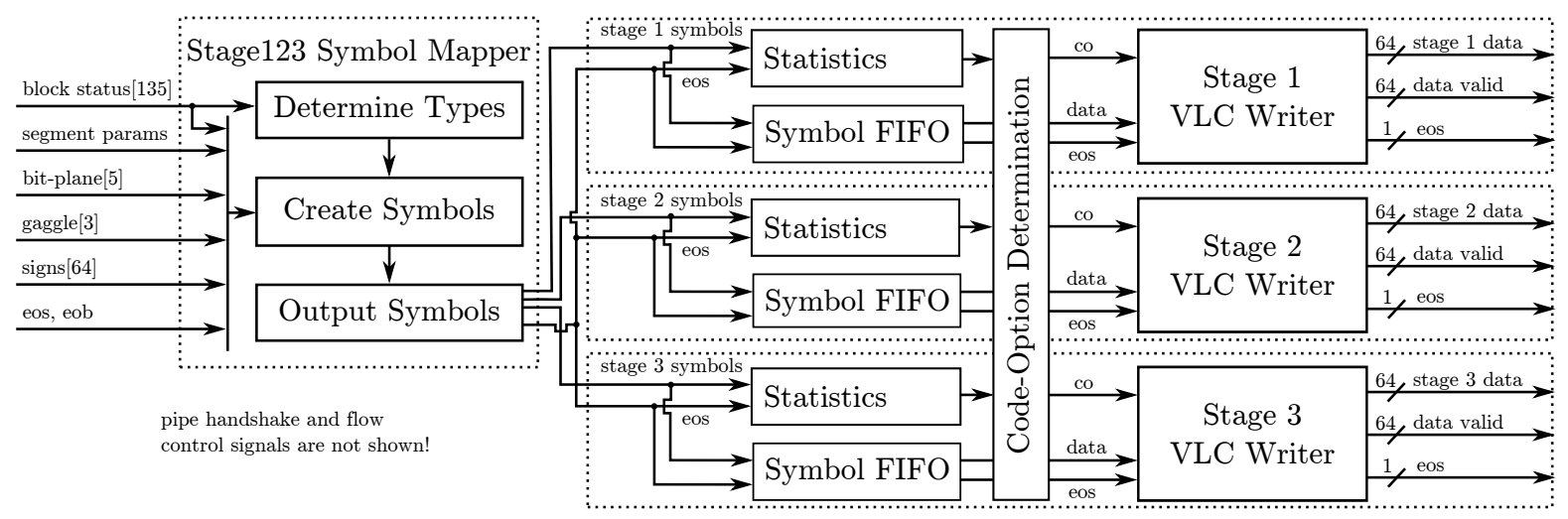

Figure 4.14: Overview on the Stages 1, 2, and 3 Coding Module.

\subsubsection{Output Muxer Module}

Depending on the image and the compression parameters, a segment consists of different bit-stream components or parts. The Output Muxer Module shown in Fig. 4.15 merges the bit-streams from up to eight coding modules into a single output bit-stream. The output vector has a width of 64 bit which corresponds to the internal width of the compression architecture: Stages 1-3 produce up to 64 bit, and stage 4 up to 63 bit of data in one clock cycle. Assuming a worst case compression ratio of 1:1 for natural (non-random) images, an output data width of $32 \mathrm{bit}$ (16 bpp input dynamic, 2 pixels per clock cycle) should be sufficient. This will need an additional FIFO to buffer the peak output data. This has not been implemented, because there are no interface requirements at the moment. In order to be standard conform, the output interface has a byte-valid signal instead of a word valid signal. A CodeWordLength of 1, 2, 3, and 4 is supported.

Furthermore, the Output Muxer Module creates the bit-stream index that is necessary to achieve scalability. The Output Muxer Module gets the information on the segment parts from the BPE Control Module.

The QuantizedDC and BitDepthAC Module and the Stage 1 to 4 Coding Modules produce sparse output data, i. e. a valid bit corresponds to each bit of the output data vector. The Segment Header Module, the Additional DC Bit-Planes Module, and the Stage 0 Coding Module does not produce spare output data. In general, the coding modules use a different width for the output data, two types of shifter modules are used to pack all valid bits into a continuous bit-stream:

- Output-Shifter Module

- Pre-Shifter Module

The Output-Shifter Module shown in Figure 4.16 first compresses the input by shifting all valid bits together. Simultaneously, it counts the number of valid bits. Based on the number of valid bits, it rotates the compressed input bits so that the current input can 


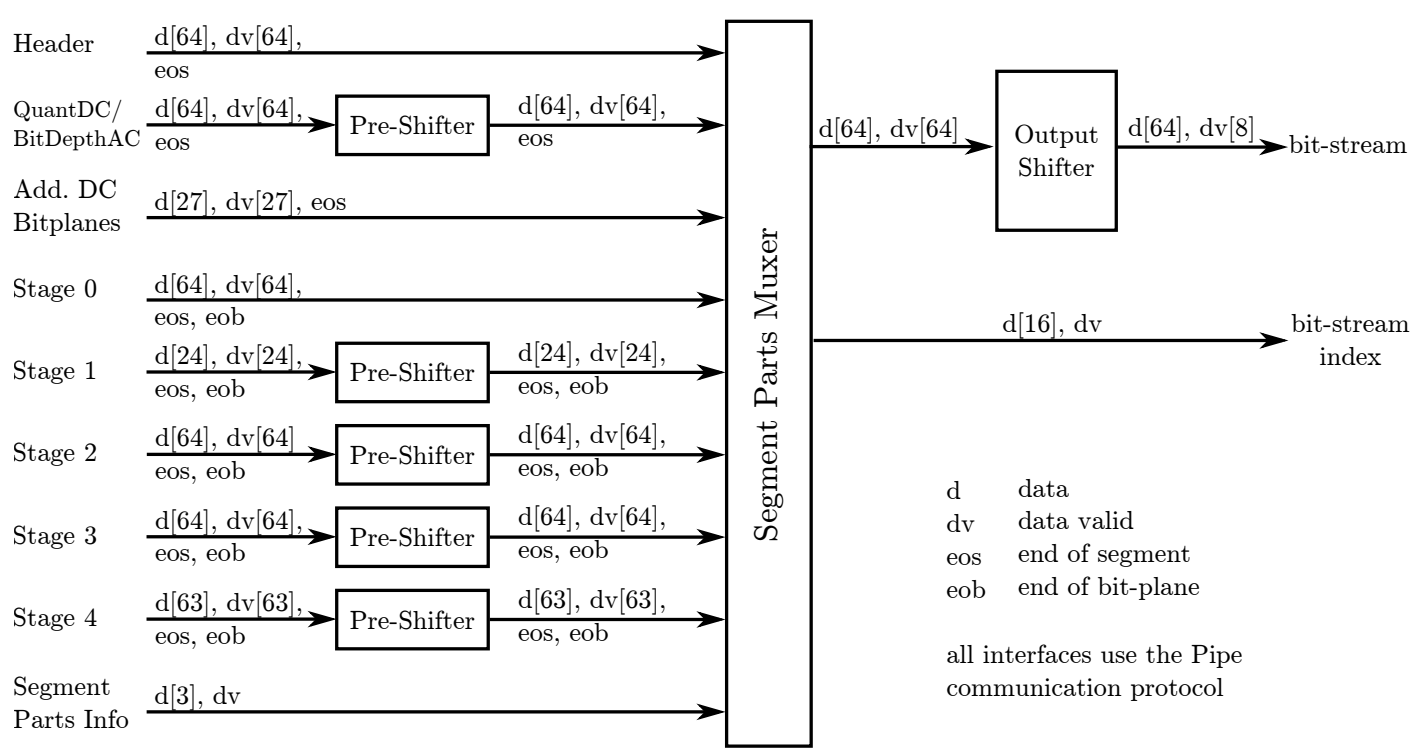

Figure 4.15: Overview on the Output Muxer Module.

easily be combined with the output buffer by logical operations. If the output buffer is full, its content forms the output of the module. The new buffer will either be empty or contain the valid bits that did not fit in the output. If there is no output, the buffer will be re-used for the next input data.

The Pre-Shifter Module is a simplification of the Output-Shifter Module. It effectively shifts on byte-level instead of bit-level, i. e. each group of 8 bits (a byte) of the output data contain at least one valid bit, except for the last data word produced by a stage coding module (end of bit-plane) or at the end of a segment. It was added to the architecture in order to reduce the input FIFO sizes of the Segment Parts Muxer Module. In principle, the Output-Shifter Module would have the same effect, but it has a significant higher FPGA logic resource consumption.

\subsubsection{Memory Controller Module}

As shown in the previous sections, the amount of FPGA internal memory may not be sufficient for wide images or large segment sizes. In this case, an external memory device must be connected to the FPGA and a corresponding memory controller must be integrated into the FPGA design. In case of the proposed architecture, it is advantageous to share the memory device between different design entities. The choice of the specific memory technology is a trade-off between flexibility, power consumption, access speed, and memory size. 


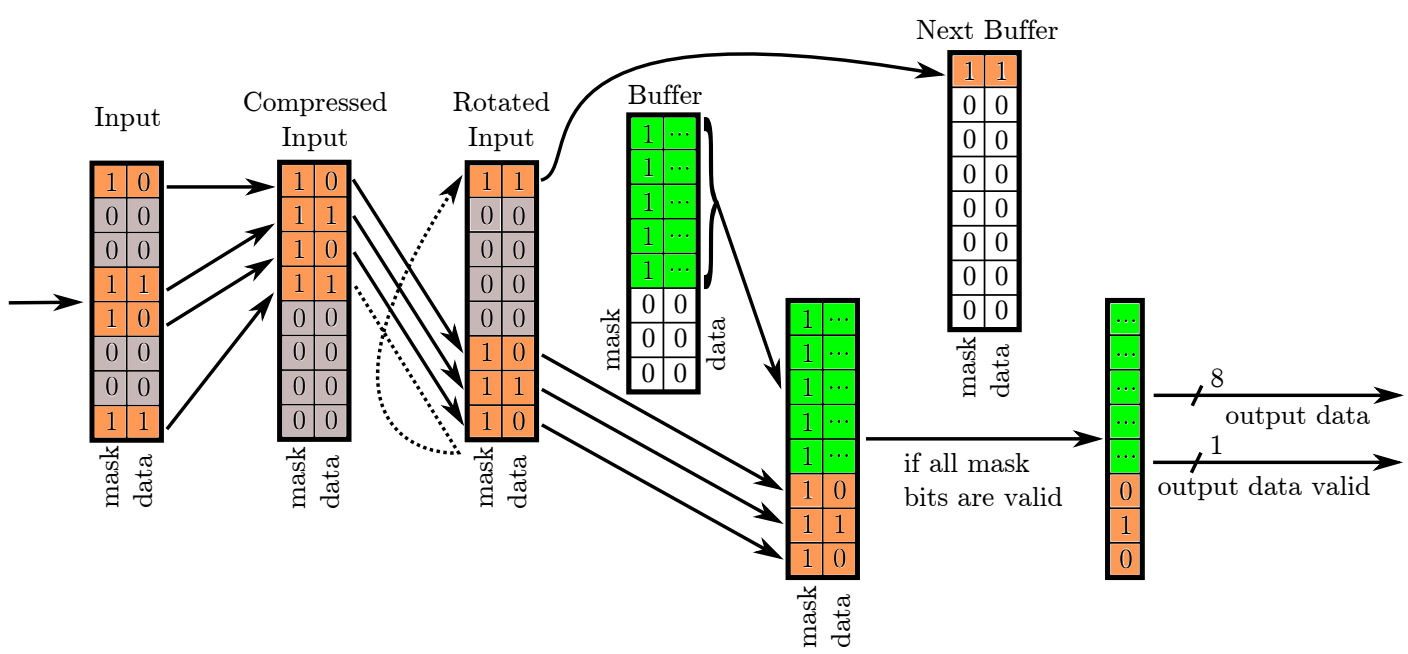

Figure 4.16: Principles of the Output-Shifter Module.

Table 4.1: Comparison of the Xilinx Virtex 5QV internal memory resources [128].

\begin{tabular}{lcc}
\hline & Slice Register & BlockRAM \\
\hline Size & $197.5 \mathrm{kbyte}$ & $1341 \mathrm{kbyte}$ \\
Clock Frequency & $100 \mathrm{MHz}$ & $100 \mathrm{MHz}$ \\
Data Throughput & $\approx 38 \mathrm{Tbyte} / \mathrm{s}\left({ }^{3}\right)$ & $\approx 523 \mathrm{Gbyte} / \mathrm{s}\left({ }^{4}\right)$ \\
Latency & some ns, same clock cycle & 1 clock cycle \\
Flexibility & very high & high \\
\hline
\end{tabular}

\subsubsection{Available Memory Resources}

Table 4.1 shows the internal memory resources of the Xilinx Virtex 5QV [128]. Either memory based on Slice Registers or BlockRAM can be used. The memory has in both cases a very high data throughput and low latency. However, due to the small size of these memories, it might not be suitable to be used by the WTM or CGM (Section 4.3.2 and Section 4.3.3.1).

Table 4.2 shows a comparison of some state-of-the-art memory technologies or devices available for space applications. Devices are available for example from the companies Cypress and 3D Plus [1-3, 27]. All these memory devices are radiation tolerant with a total ionizing dose (TID) of at least $50 \mathrm{Krad}$. Since PCB area is relatively expensive, a single memory device with a data-throughput in the order of several Gbyte/s is desirable. QDRII+ SRAM is very promising because it has a high data throughput and a low latency. In addition, it guarantees the data throughput at any time, because it does not require refresh cycles.

\footnotetext{
${ }^{3} 1580 \mathrm{kbit} \cdot 2 \cdot 100 \mathrm{MHz}=316000 \mathrm{Gbit} / \mathrm{s}=39500 \mathrm{Gbyte} / \mathrm{s} \approx 38 \mathrm{Tbyte} / \mathrm{s}$

${ }^{4} 298 \cdot 72 \mathrm{bit} \cdot 2 \cdot 100 \mathrm{MHz}=4291200 \mathrm{Mbit} / \mathrm{s}=536400 \mathrm{Mbyte} / \mathrm{s} \approx 523 \mathrm{Gbyte} / \mathrm{s}$

${ }^{5} 36 \mathrm{bit} \cdot 250 \mathrm{MHz} \cdot 2 \cdot 2=36 \mathrm{Gbit} / \mathrm{s}=4.5 \mathrm{Gbyte} / \mathrm{s}$
} 
Table 4.2: A comparison of some state-of-the-art memory technologies available for space applications.

\begin{tabular}{lcccc}
\hline & $\begin{array}{c}\text { QDRII+ } \\
\text { SRAM }\end{array}$ & $\begin{array}{c}\text { DDR2 } \\
\text { SDRAM }\end{array}$ & $\begin{array}{c}\text { DDR2 } \\
\text { SDRAM }\end{array}$ & $\begin{array}{c}\text { NAND } \\
\text { Flash }\end{array}$ \\
\hline Vendor & Cypress & 3D Plus & 3D Plus & 3D Plus \\
Part & CYRS1544AV18 & 2D4G08US4283 & 2D4G72UB3478 & FN32G64VB8263 \\
TID [Krads (Si)] & 300 & 50 & 50 & 50 \\
Size [per chip] & $9 \mathrm{Mbyte}$ & $512 \mathrm{Mbyte}$ & $576 \mathrm{Mbyte}$ & $8192 \mathrm{Mbyte}$ \\
Configuration & $2 \mathrm{Mx36}$ & $512 \mathrm{Mx} 8$ & $64 \mathrm{Mx} 72$ & $512 \mathrm{Mx64}$ \\
Clock Frequency & $250 \mathrm{MHz}$ & $200 \mathrm{MHz}$ & $200 \mathrm{MHz}$ & $40 \mathrm{MHz}$ \\
Max. Throughput & $4.5 \mathrm{Gbyte} / \mathrm{s}\left({ }^{5}\right)$ & $400 \mathrm{Mbyte} / \mathrm{s}$ & $3.6 \mathrm{Gbyte} / \mathrm{s}$ & $320 \mathrm{Mbyte} / \mathrm{s}$ \\
Latency & 2 clock cycles & $\approx 4-7 \mathrm{clock}$ & $\approx 4-7 \mathrm{clock}$ & $\approx 1 \mathrm{~ms}$ \\
& burst r/w & cycles & cycles & \\
Remarks & - & needs refresh & needs refresh & volatile \\
& & cycles & cycles & \\
\hline
\end{tabular}

\subsubsection{Concept for Using External Memory}

When dealing with external memory such as QDRII+ SRAM, problems arise that do not exist when using FPGA internal BlockRAM or registers:

- relatively high read and write latencies due to the complicated access to the physical memory

- different clock domains between data processing and physical memory

- constant bus width of the physical memory which does not fit to the width of the data processing

- the physical memory is much faster than required by a single module inside the design

- complex handling of memory addresses

Some of these problems can be solved by combining the First In, First Out (FIFO) principle with a SRAM memory controller. The Hardware Operating System presented in Section 2.3.3 abstracts external memory by a so-called Pipe Delay Module. The Pipe Delay Module implements essentially a Pipe FIFO: The module has a Pipe input and a Pipe output. The Pipe data written to the input is presented at the output. The module uses external memory to buffer the Pipe frame data (Figure 4.17). The amount of data that can be buffered in the module depends on the attached memory size.

In the original variant of the Pipe Delay Module presented in [64], SDRAM is organized as a ring buffer together with pointers to the first free and to the last used memory address. For the separation of the individual Pipe frames, a FIFO stores the frame number as 


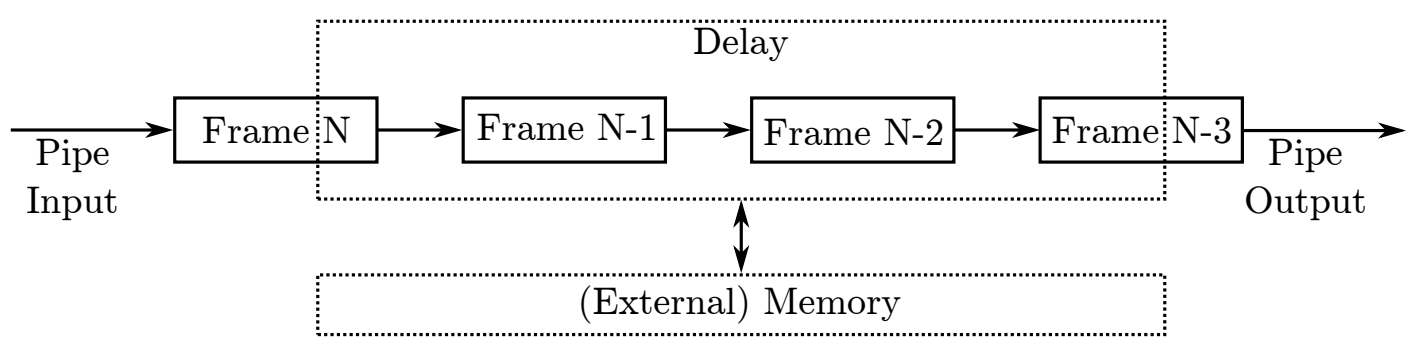

Figure 4.17: Pipe Delay Module: The data frames are written to the input. One or more frame may be stored inside the module. External memory is used to for buffering. A previous (or the same) frame can be read on the output port.

well as the address of the last data word of the frame. One limitation of the module is that the interface data width is not independent of the interface width of the memory and only Pipe frame with a width up to the width of the memory can be stored. If the width of the Pipe is smaller than that of the memory module, memory remains unused.

Since QDRII+ SRAM has a very high data bandwidth, it is possible to share the bandwidth between several Pipe Delay Modules. The approach of the simple Pipe Delay Module was extended as follows: The so-called Multi-Pipe Delay Module (MDM) implements multiple Pipe FIFOs whose data are stored together in a single memory. The MDM has a hierarchical structure and uses a standard (QDRII+) SRAM controller (PHY) as a basis. Based on the PHY, a multi-port controller is developed that allows reading and writing over several channels. It uses fixed priority scheduling, i. e. channels with a high priority get rather access to the memory than channels with a lower priority. The bit width adaption between the Pipes and the memory and the clock domain crossing is performed by pipe_output_converter and pipe_input_converter ${ }^{6}$ modules also used in [64]. Figure 4.18 shows the schematic structure of a MDM. If the actual FPGA board has more than one (QDRII+) SRAM module, multiple MDMs can be instantiated.

\subsubsection{Integration Into the Design}

Since the memory sizes for the DWT column transform or the CGM will exceed the FPGA's internal memory resources, the corresponding VHDL entities in the design use either BlockRAMs or FIFOs based on BlockRAM. The maximum image width or row size determines the size of the memory in the particular modules. If only the FPGA's internal memory resources are used, and if the maximum row size exceeds a certain threshold, the design will not fit into the FPGA. From the engineering point of view, it is often not trivial to replace internal by external memory, since signals must be routed through the complete design hierarchy. The situation is further complicated if the decision as to whether internal or external memory is used is made based on VHDL generics.

\footnotetext{
${ }^{6}$ These modules have a Pipe input and output and basically implement an asynchronous FIFO and bit-width adaption.
} 


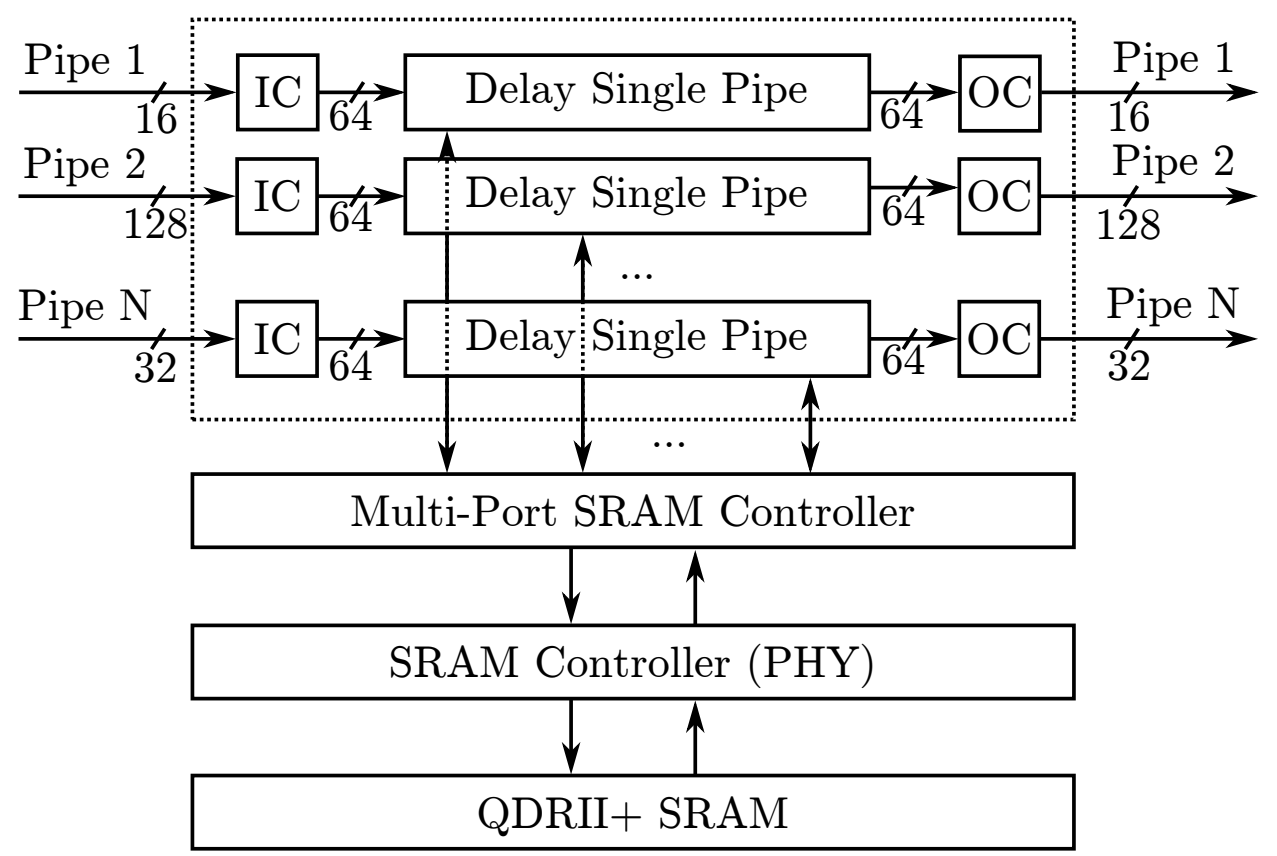

Figure 4.18: Multi-Pipe Delay Module with SRAM memory controller ( $\mathrm{IC}=$ pipe_input_converter, $\mathrm{OC}=$ pipe_output_converter).

With the Hardware Operating System and its Structure Compiler presented in [64] it is possible to abstract external memory (e.g. QDRII+ SRAM) in such a way that it can be used like FPGA's internal BlockRAM. In principle the Structure Compiler can substitute a generic instance of a memory either by BlockRAM or external memory. In the latter case, it also adds the required signal to the external memory. This approach is used in the architecture to implement a unified memory interface. Thus, BlockRAM can easily be replaced by external memory. Figure 4.19 shows this concept.

The benefits of using a unified memory interface are that the design can be developed and verified independent of the specific external memory technology. It can be used on all platforms for which the corresponding interfaces are available. Furthermore, with

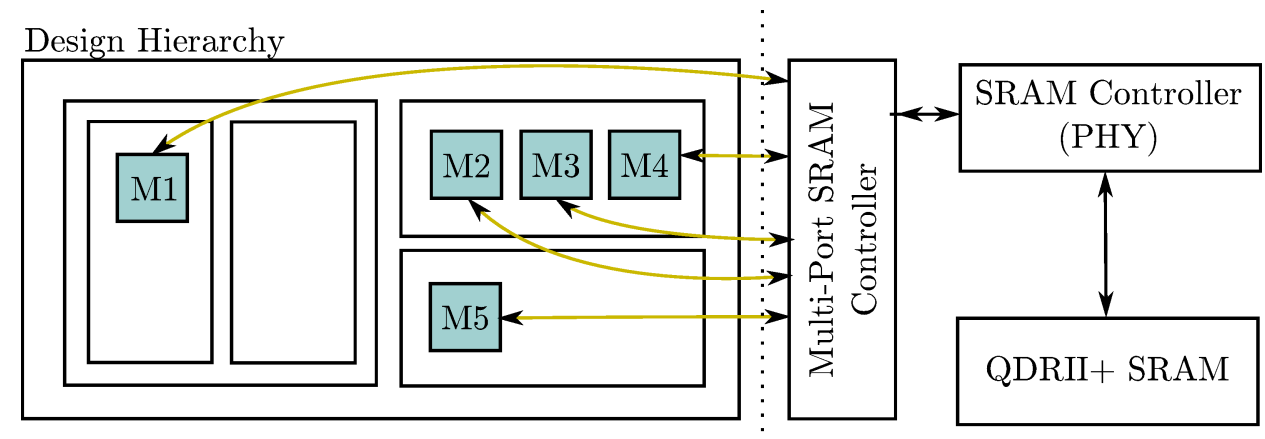

Figure 4.19: Concept of using external memory. 
the use of the hardware operating system, no signal routing of memory-specific signals between top- and bottom-level is necessary in the user application.

\subsection{Summary}

This chapter has presented the design of an FPGA-based architecture for real-time satellite image data compression. The architecture can be used on recent space-qualified FPGAs. The quantitative requirements were first elicited and analyzed. Then, the structure of the proposed architecture and their modules were presented. The CCM is used for TM and TC. The image data is sent directly to the WTM that uses a line-based architecture. The calculated DWT coefficients are grouped to blocks and segments by the CGM. The BPEM gets the segments from the CGM, codes the bit-stream, and creates an index that can be used for later re-assembling of the bit-stream. Most of the requirements listed in Section 4.1 are fulfilled by design. Performance requirements regarding data compression throughput and resource or power consumption will be investigated in Chapter 5. 



\section{Experimental Evaluation}

The CCSDS 122.0-B-1 compression algorithm presented in Section 2.4 and the extensions presented in Chapter 3 have been successfully implemented on reconfigurable hardware that is qualified for space applications. The architecture presented in Chapter 4 was implemented in VHDL and integrated into an existing development and testing framework (see Section 2.3.3). In order to make meaningful statements about design feasibility and resource utilization, a new FPGA board had to be integrated into this environment. Therefore, a PCIe core [132] was integrated and the software drivers were adapted. The FPGA design was verified with ModelSim [80].

In this chapter the results of the thesis are presented and discussed. The methodology is presented in Section 5.1. In Section 5.2, results concerning the extensions are presented. The speed and the data throughput of the FPGA implementation are presented in Section 5.3. The FPGA resource consumption and the maximum clock frequency are discussed in Section 5.4. Since the power consumption is a critical property for space applications, it was determined and is presented in Section 5.5. A comparison of the work presented in this thesis with a GPGPU implementation [89], and with other implementations is shown in Section 5.6. In order to get a better understanding of the impact of image compressing using the CCSDS 122.0-B-1 standard, corresponding investigations were carried out and presented in Section 5.7. A short summary of this chapter is given in Section 5.8.

\subsection{Methodology}

Test images and two reference implementations of the CCSDS 122.0-B-1 standard from the University of Nebraska [118] and from the Universitat Autonoma de Barcelona [44] were investigated. An implementation based on $\mathrm{C}++$ and Matlab [110] was also developed for a better understanding of the algorithm and for the implementation of the proposed extensions. The algorithm and its extensions were implemented in $\mathrm{C}++$. The functionality of the encoder and decoder was provided in Matlab via the MATLAB Executable (MEX) interface. The software implementation has a data throughput of approximately $2 \mathrm{Mbyte} / \mathrm{s}$, which is not suitable for high-speed and real-time image data compression.

In order to assess the results better, a GPGPU implementation of the CCSDS 122.0-B-1 standard, based on a NVidia Compute Unified Device Architecture (CUDA) graphics card, has also been developed by a master student [89]. Basis of the GPGPU implementation 
was the CPU implementation that was developed for this thesis. The master student mainly ported the entropy encoder (BitPlaneEncoder) to a GPGPU. The performance measurements were carried out in co-operation and were evaluated together. Comparative results for the GPGPU implementation are presented in Section 5.6.

For this thesis, a fully synthesizable hardware implementation in VHDL was developed. Care has been taken to ensure high re-usability and low platform-dependency, i. e. the proposed architecture is not fixed to a specific architecture. The Hardware Operation System [64] was used for this purpose. The Matlab implementation was used to test the algorithm and its extensions, and to generate test data for the Modelsim hardware simulation. The complete CCSDS test data set was used to validate the hardware architecture. The hardware simulation produces (interim) results which are then validated in Matlab. After the functional verification was successfully performed, some experiments were conducted to determine the data throughput, the resource consumption, the maximum clock frequency, and the power consumption.

The used software and hardware components are as follows:

\section{Software}

- Visual Studio 2013 / GCC 4.7.2 for C/C++ development

- MATLAB R2013a for validation, test data generation

- Xilinx Design Suite 14.7 for FPGA synthesis

- Modelsim SE-64 10.1b for hardware simulation

- Synopsys FPGA Synthesis I-2013.09-1 for FPGA synthesis

\section{Hardware}

- Xilinx SP605 reference design board for initial tests

- HiTechGlobal HTG-K700 FPGA board with Xilinx Kintex-7 325T FPGA, hardware demonstrator

- Dell PC with Intel Core i7-3770 CPU, 8 Gbyte RAM, running Ubuntu 14.04.2 LTS, for CPU reference

- NVIDIA® GeForce® GTX 670 GPU, CUDA version 5.5, NVIDIA driver version 331.113, NVIDIA CUDA compiler nvcc, for GPGPU reference

\subsection{Overhead of the Index Table for ROI Coding}

Extensions for multi-spectral compression and ROI technology as well as an approach to re-assemble the bit-stream after compression have been presented in Chapter 3 . 
Table 5.1: Size of the index compared to the size of the input image (in \%)

\begin{tabular}{lrrrrrr}
\hline & $S=16$ & $S=32$ & $S=64$ & $S=128$ & $S=256$ & $S=512$ \\
\hline coastal_b1 & 4.19 & 2.45 & 1.44 & 0.86 & 0.48 & 0.27 \\
marstest & 6.12 & 3.48 & 1.98 & 1.13 & 0.65 & 0.39 \\
ice_2kb1 & 5.95 & 3.39 & 1.89 & 1.06 & 0.58 & 0.32 \\
pleiades_portdebouc_b3 & 7.93 & 4.58 & 2.59 & 1.44 & 0.81 & 0.47 \\
pleiades_portdebouc_pan & 7.37 & 4.19 & 2.35 & 1.30 & 0.72 & 0.39 \\
sar & 8.08 & 4.73 & 2.70 & 1.55 & 0.90 & 0.52 \\
spot-la_panchr & 5.37 & 3.06 & 1.74 & 0.97 & 0.55 & 0.31 \\
\hline average & 6.43 & 3.70 & 2.10 & 1.19 & 0.67 & 0.38 \\
\hline
\end{tabular}

ROI coding can be achieved by controlling the compression parameters segment-wise. In order to obtain an optimal overlap between desired ROI and encoded ROI, the segment size $S$ can be changed for any segment down to just one block. The approach was implemented in hardware as well as in a software reference model. Figure 3.1b shows the image marstest with a ROI in the center of the image. The image consists of 4096 blocks. If the segment size is $S=16$ blocks, the compressed bit-stream consists of 256 segments.

The additional index table that has to be stored for scalability effectively reduces the compression efficiency, but it does not have to be transmitted to the ground station, since the bit-stream is re-assembled and the transfer frame is generated on-board the spacecraft. Table 5.1 shows the ratio of the index size to the input image size (in percentage) for some images with a dynamic range of 8 bit to 16 bit. At least one image for each dynamic range value of the CCSDS data set was selected. A segment size of $S=128$ blocks leads to an average index size of $1.19 \%$ of the input image size for the examined images. The overhead decreases when the segment size $S$ increases. This is obvious because each entry in the index table contains coded bits from all blocks in a segment, in other words, the maximum number of entries in the index table does not depend on the segment size $S$ (see Equation 3.6). There is a variation in the relative size of the index table. This can be explained by the fact that with constant compression parameters, the number of index entries only depends on the bit-depth of the AC coefficients. These in turn depend on the image content. An image with little high frequency information has a lower value for BitDepthAC and thus fewer index entries. Taking in consideration the assumption that data transmission to the ground station is more critical than on-board storage capacity, $1.19 \%$ (max. $1.44 \%$ for the tested images) additional memory for the index table is acceptable.

\subsection{Data Compression Throughput}

The data compression throughput of the FPGA implementation was measured via simulation in ModelSim at a clock frequency of $100 \mathrm{MHz}$ (integer DWT) or $55 \mathrm{MHz}$ 
Table 5.2: Data compression throughput for lossless compression and a segment size of $S=128$ blocks (Integer only, $100 \mathrm{MHz}$ ).

\begin{tabular}{|c|c|c|c|c|c|c|}
\hline \multirow[t]{2}{*}{ Image } & \multicolumn{2}{|l|}{ Size } & \multirow{2}{*}{$\begin{array}{c}\text { Time } \\
{[\mathrm{ms}]}\end{array}$} & \multicolumn{3}{|c|}{ Throughput } \\
\hline & {$[\mathrm{w} \times \mathrm{h} \times \mathrm{bpp}]$} & [kbyte] & & [Mbyte/s] & {$[\mathrm{Mpx} / \mathrm{s}]$} & [px/cycle] \\
\hline coastal_b1 & $1024 \times 1024 \times 8$ & 1024 & 5.40 & \multicolumn{2}{|c|}{194.35} & 1.94 \\
\hline coastal_b2 & $1024 \times 1024 \times 8$ & 1024 & 5.40 & \multicolumn{2}{|c|}{194.47} & 1.94 \\
\hline coastal_b3 & $1024 \times 1024 \times 8$ & 1024 & 5.40 & \multicolumn{2}{|c|}{194.26} & 1.94 \\
\hline coastal_b4 & $1024 \times 1024 \times 8$ & 1024 & 5.39 & \multicolumn{2}{|c|}{194.61} & 1.95 \\
\hline coastal_b5 & $1024 \times 1024 \times 8$ & 1024 & 5.39 & \multicolumn{2}{|c|}{194.46} & 1.94 \\
\hline coastal_b6h & $512 \times 512 \times 8$ & 256 & 1.39 & \multicolumn{2}{|c|}{188.49} & 1.88 \\
\hline coastal_b61 & $512 \times 512 \times 8$ & 256 & 1.39 & \multicolumn{2}{|c|}{188.95} & 1.89 \\
\hline coastal_b7 & $1024 \times 1024 \times 8$ & 1024 & 5.40 & \multicolumn{2}{|c|}{194.34} & 1.94 \\
\hline coastal_b8 & $2048 \times 2048 \times 8$ & 4096 & 21.29 & \multicolumn{2}{|c|}{197.01} & 1.97 \\
\hline europa $\overline{3}$ & $557 \times 600 \times 8$ & 327 & 1.81 & \multicolumn{2}{|c|}{185.05} & 1.85 \\
\hline marstest & $512 \times 512 \times 8$ & 256 & 1.38 & \multicolumn{2}{|c|}{190.35} & 1.90 \\
\hline lunar & $512 \times 512 \times 8$ & 256 & 1.40 & \multicolumn{2}{|c|}{187.71} & 1.88 \\
\hline spot-la_b3 & $500 \times 500 \times 8$ & 245 & 1.35 & \multicolumn{2}{|c|}{184.75} & 1.85 \\
\hline spot-la_panchr & $1000 \times 1000 \times 8$ & 977 & 5.15 & \multicolumn{2}{|c|}{194.30} & 1.94 \\
\hline foc & $1024 \times 512 \times 8$ & 512 & 2.72 & \multicolumn{2}{|c|}{192.98} & 1.93 \\
\hline ice_2kb1 & $2048 \times 2048 \times 10$ & 5120 & 21.30 & 246.18 & 196.94 & 1.97 \\
\hline ice_2kb4 & $2048 \times 2048 \times 10$ & 5120 & 21.29 & 246.29 & 197.03 & 1.97 \\
\hline india_2kb1 & $2048 \times 2048 \times 10$ & 5120 & 21.33 & 245.78 & 196.62 & 1.97 \\
\hline india_2kb4 & $2048 \times 2048 \times 10$ & 5120 & 21.33 & 245.82 & 196.66 & 1.97 \\
\hline ocean_2kb1 & $2048 \times 2048 \times 10$ & 5120 & 21.31 & 246.08 & 196.86 & 1.97 \\
\hline ocean_2kb4 & $2048 \times 2048 \times 10$ & 5120 & 21.27 & 246.50 & 197.20 & 1.97 \\
\hline landesV_G7_10b & $454 \times 2381 \times 10$ & 1320 & 5.67 & 238.39 & 190.71 & 1.91 \\
\hline marseille_G6_10b & $528 \times 1856 \times 10$ & 1197 & 5.10 & 240.23 & 192.19 & 1.92 \\
\hline pleiades_portdebouc_b3 & $1376 \times 320 \times 12$ & 645 & 2.41 & 274.54 & 183.03 & 1.83 \\
\hline pleiades_portdebouc_pan & $1400 \times 5504 \times 12$ & 11288 & 39.10 & 295.61 & 197.07 & 1.97 \\
\hline solar & $1024 \times 1024 \times 12$ & 1536 & 5.43 & 289.93 & 193.28 & 1.93 \\
\hline sun_spot & $512 \times 512 \times 12$ & 384 & 1.41 & 278.00 & 185.33 & 1.85 \\
\hline wfpc & $800 \times 800 \times 12$ & 938 & 3.34 & 287.28 & 191.52 & 1.92 \\
\hline p160_b_f & $2048 \times 2048 \times 16$ & 8192 & 21.46 & 390.83 & 195.42 & 1.95 \\
\hline sar & $512 \times 512 \times 16$ & 512 & 1.42 & 368.16 & 184.08 & 1.84 \\
\hline noise8 & $512 \times 512 \times 8$ & 256 & 1.41 & \multicolumn{2}{|c|}{185.35} & 1.85 \\
\hline noise10 & $512 \times 512 \times 10$ & 320 & 1.42 & 230.29 & 184.23 & 1.84 \\
\hline noise12 & $512 \times 512 \times 12$ & 384 & 1.43 & 274.88 & 183.26 & 1.83 \\
\hline noise14 & $512 \times 512 \times 12$ & 384 & 1.44 & 318.93 & 182.25 & 1.82 \\
\hline noise16 & $512 \times 512 \times 16$ & 512 & 1.46 & 358.87 & 179.43 & 1.79 \\
\hline noise17 & $512 \times 512 \times 17$ & 576 & 1.50 & 349.31 & 174.65 & 1.75 \\
\hline noise18 & $512 \times 512 \times 18$ & 576 & 1.54 & 382.00 & 169.78 & 1.70 \\
\hline
\end{tabular}


(FP DWT). A simulation was chosen in order to get the data throughput unaffected by any interface limitation. The design has an input Pipe for the input data and an output Pipe for the compressed data. In the simulation, the input image data as well as the output bit-stream interface are always ready, i. e. the output-busy signal is never raised and propagated to the input (see Section 2.3.3.3). The simulation perfectly reflects the timing behavior inside the FPGA. The compression time is the duration where at least one of the interfaces is active, i. e. from rising input request to falling output confirm. The simulation is made for various images from Earth observation and remote sensing presented in Table B.1 and some generated noise images. The images have a dynamic range of 8 bit to 18 bit. The image sizes are between $512 \times 512 \mathrm{px}$ and $1400 \times 5504 \mathrm{px}$. All images are compressed lossless (Integer DWT) or near-lossless (FP DWT). The complete dataset consists of 34 images $(\approx 77$ Mbyte). For a integer-only architecture, all images were compressed in the hardware simulation to get a precise time measurement without any interface delays. For comparison, the simulation was also done for a FP+integer architecture using the FP DWT. The resulting bit-streams were validated with a functionally identical software implementation of the algorithm. Table 5.2 and Table 5.3 show the simulation results for both variants of the architecture. The average data compression throughput for all images is approximately $190 \mathrm{Mpx} / \mathrm{s}(192 \mathrm{Mpx} / \mathrm{s}$ for the CCSDS reference images shown in Section B.4). The busy signal of the input Pipe was never active during compression, i. e. the input port of the compression core processes 2 pixels per clock cycle at any time. Again, the time for the compression was measured between input-request and output-confirm and includes the pipeline latency of the complete compression chain. There are two phases that are included in the measurement:

- The time at the beginning of an image where no output data can be produced because the first segment must be transformed completely before coding.

- The time at the end of an image where the remaining (already transformed) segments are coded.

The significance of these 2 phases decreases with larger images or segment sizes. It is evident that a higher dynamic range of the input images has almost no impact on the data compression throughput with respect to the pixel rate. As would be expected, the coding time strongly depends on the spatial size of the input data and not on the dynamic of the images. This can be explained by the fact that the entropy encoder architecture is optimized for a dynamic range of 16 bit and can continuously process the output of the wavelet transform module.

The last columns of the Table 5.2 and Table 5.3 show the performance independent of the clock frequency. Using the FP DWT will lead to almost identical results in terms of the number of clock cycles needed for compression, because the FP architecture has a very similar timing behavior. The only difference is the maximum clock frequency which is approximately $50 \%$ of the architecture without FP support. The compression throughput in terms of pixels per clock cycle is between $1.80 \mathrm{px} /$ cycle and $1.97 \mathrm{px} / \mathrm{cycle}$ for 
Table 5.3: Data compression throughput for near-lossless compression and a segment size of $S=128$ blocks (FP and Integer DWT architecture, FP used, $55 \mathrm{MHz}$ ).

\begin{tabular}{lcccc}
\hline Image & Time & \multicolumn{3}{c}{ Throughput } \\
& {$[\mathrm{ms}]$} & {$[\mathrm{Mbyte} / \mathrm{s}]$} & {$[\mathrm{Mpx} / \mathrm{s}]$} & {$[\mathrm{px} / \mathrm{cycle}]$} \\
\hline coastal_b4 & 9.86 & 106.33 & 1.93 \\
marstest & 2.61 & 100.36 & 1.82 \\
foc & 5.01 & \multicolumn{2}{c}{104.65} & 1.90 \\
sar & 2.65 & 198.19 & 99.10 & 1.80 \\
ice_2kb4 & 3.87 & 135.34 & 108.27 & 1.97 \\
\hline
\end{tabular}

natural images for both variants.

The BitPlaneEncoder module is designed to ensure continuously image compression of any image, i. e. the theoretical maximum number of bit-plane loop iterations (up to 21 for 16 bit unsigned images [25]) and the maximum number of symbols at any stage and block without back-propagation of a busy signal to the DWT module. For real images, this worst-case will hardly happen. The BitPlaneEncoder module starts at bit-plane $b=$ BitDepthAC -1 . If the value $b$ is low due to a low dynamic of the AC coefficients or quality controlling parameters such as BitPlaneStop or SegByteLimit, the BitPlaneEncoder must effectively wait for wavelet coefficients (the Coefficient Grouping Module also does not limit the data throughput). Since the data throughput is limited by the DWT module, the theoretical maximum is at $2 \mathrm{px}$ per clock cycle. The real throughput is slightly lower because the of the boarder handling of the wavelet transform (pixels must be mirrored). This introduces 4 additional clock cycles at the beginning and end of each line for row and column transform and for each decomposition level.

Noise images were tested for the following reasons. These images have the effect that the amount of data increases due to the compression. Furthermore, the compression system is under stress, i. e. a high or maximum amount of data is produced in the coding sub-modules. This might produce errors that do not occur in normal operation. It is worth mentioning that noise images with a dynamic range of 17 bit or 18 bit could be compressed without errors.

\subsection{Resource Consumption and Maximum Clock Frequency}

The maximum clock frequency of the FPGA implementation depends on the length of the critical path, which consists of setup and hold constraints of the target architecture's memory elements (registers, flip-flops) and proportional delay of the sequential logic between these elements. In FPGA design, sequential logic most often dominates the length of the critical path. The length of the critical path itself can be shortened by adding 
additional registers or pipeline stages. This in turn increases the resource consumption of the design.

In this section, the resource consumption and the maximum clock frequency of the individual modules of the compression architecture are presented. The evaluation is performed using the following parameters:

- Internal precision of the integer/floating point arithmetic: 24 bit

- Supported wavelet transforms: Integer DWT only, Integer + FP DWT

- Maximum image width: $I_{w}=1024 \mathrm{px}, I_{w}=2048 \mathrm{px}$ and $I_{w}=4096 \mathrm{px}$

- Maximum segment size: $S=128$ Blocks

The Xilinx Virtex-5 XC5FX130T-1 was selected since there is the radiation hardened version XQR5VFX130 with the same number of resources which is qualified for space applications. It is assumed that the Xilinx Kintex-7 XC7K325T-2 represents a potential future space qualified FPGA in terms of resources and speed.

The FPGA resource consumption was determined by synthesizing and mapping the design to the particular target architecture. This was done with the Xilinx design tools (see above) and their architecture-specific default parameters.

\subsubsection{Internal Precision of the Arithmetic}

In order to reduce the demands on temporary memory and to reduce the FPGA resource consumption, the floating point module was realized using only 24 bit instead of single precision (32 bit). This approach was also used in [71]. The number of bits to represent the significand was reduced from 23 bit to 15 bit. The number of bits to represent the exponent remain 8 bit. The sign needs only one bit (see also [51]). Thus, the width of the floating point data path in the wavelet transform module can be reduced to 24 bit, which perfectly fits the internal data width needed for the integer transform.

This modification has almost no impact on the compression performance. Figure 5.1 compares the 24-bit to the 32-bit floating-point DWT. Both curves are so close together that little difference is discernible.

\subsubsection{Virtex-5 Synthesis Results}

Tables 5.4, 5.5, and 5.6 show the absolute number and the percentage of used internal resources of the Virtex 5 XC5FX130T-1 for a maximum image width of 1024 px, 2048 px and $4096 \mathrm{px}$, respectively. For a given segment size $S$, the usage of slice registers, LUTs and LUTRAMs is approximately $34 \%, 46 \%$ and $8 \%$, respectively, independent of the maximum image width $I_{w}$. As already implied in Section 4.3, there is a dependence 


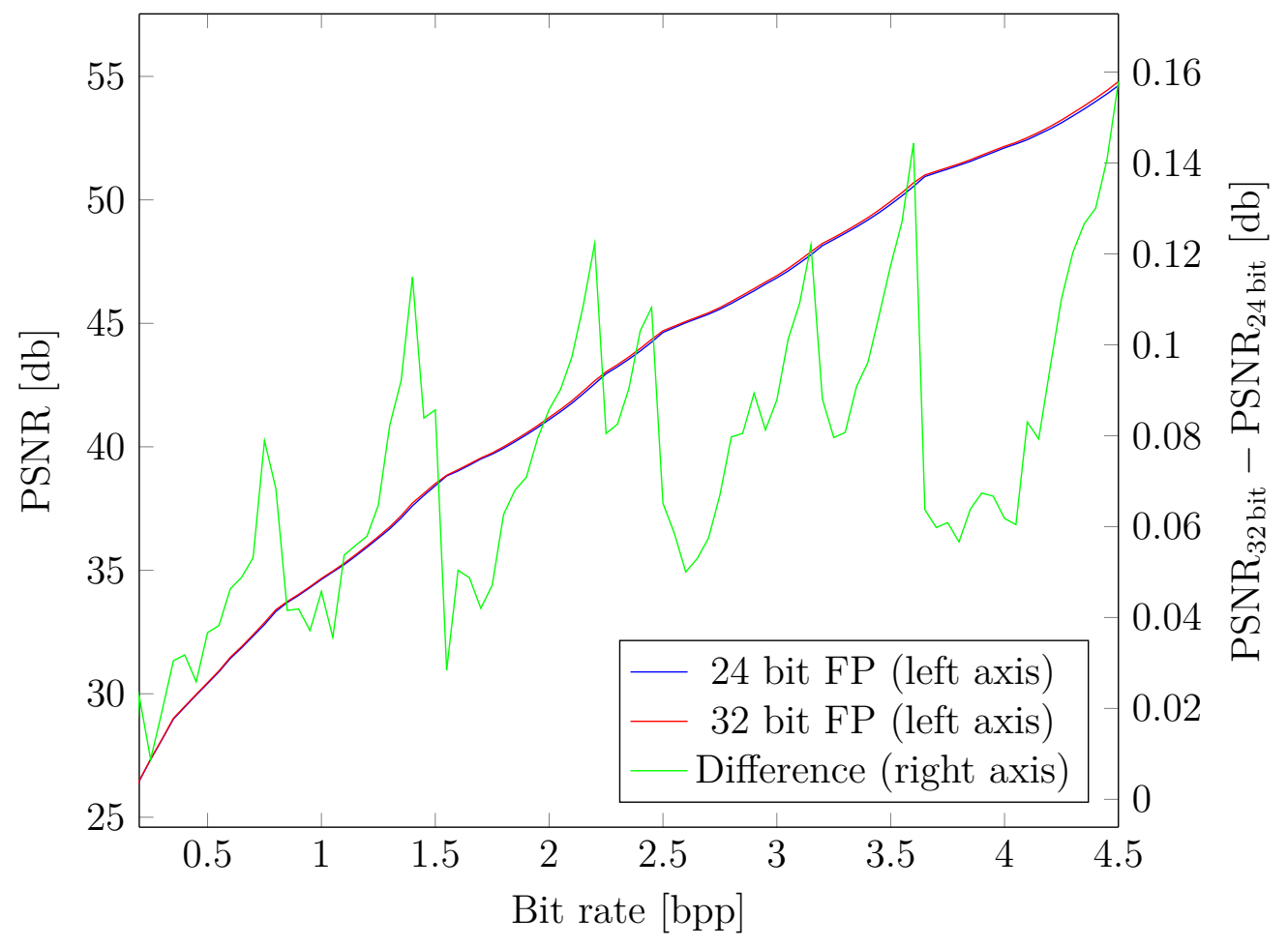

Figure 5.1: Qualitative comparison of the 24-bit and 32-bit floating-point DWT. The image marstest image was used.

between the maximum image width and the amount of memory or the number of BlockRAMs. The total number of BlockRAMs is between $50 \%$ and $77 \%$. An image width of more than $4096 \mathrm{px}$ requires external SRAM. For $I_{w}=8192 \mathrm{px}$, the number of BlockRAMs is approximately 330, which does not fit into the desired FPGA (298 BlockRAMs).

The two algorithmic main modules, the DWT and the BitPlaneEncoder module, consume the most slice registers and LUTs. The resource consumption of the DWT module depends almost exclusively on the maximum width of the input images and is constant in terms of slice registers, LUTs, and LUTRAMs. The number of BlockRAMs increases with the width of the image, because it is used to buffer lines of image pixels or coefficients. The high number of LUTs used for the DWT module is caused by arithmetic operations. The Coefficient Grouping and the Output module are not processing any part of the compression algorithm, but they are needed to achieve a high data throughput. The module uses BlockRAMs for buffering of wavelet coefficients (up to 4 lines of each decomposition level must be buffered) and segment double-buffering. The Compression Control module has insignificant resource consumption in all configurations.

The target frequency of $100 \mathrm{MHz}$ could not be reached on the Virtex-5 FPGA when using the internal DSPs for the FP DWT. Table 5.7 shows the synthesis results for a maximum image width of $I_{w}=4096 \mathrm{px}$ and segment size of 128 blocks. The maximum 
design frequency is $55.64 \mathrm{MHz}$, which corresponds to a maximum data compression throughput of approximately $111 \mathrm{Mpx} / \mathrm{s}$ (or $222 \mathrm{Mbyte} / \mathrm{s}$ for 16 bit images). Since also the resource consumption of the discrete wavelet transform module is significantly higher if the floating point module is enabled, a solution to solve both problems is to use a fixed point transform.

Table 5.4: Resource consumption of the architecture on a Virtex-5 XC5FX130T-1 for $d=24$ bit, $I_{h}=I_{w}=1024 \mathbf{p x}$ and $S=128$ Blocks (Integer DWT only). The maximum frequency of the design on this particular FPGA is $125.33 \mathrm{MHz}$.

\begin{tabular}{lrrrrrrrr}
\hline \multirow{2}{*}{ Module } & \multicolumn{2}{c}{ Slice Reg. } & \multicolumn{2}{c}{ LUT } & \multicolumn{3}{c}{ LUTRAM } & \multicolumn{2}{c}{ BlockRAM } \\
& abs. & $\%$ & abs. & $\%$ & abs. & $\%$ & abs. & $\%$ \\
\hline Compr. Control & 124 & 0.15 & 191 & 0.23 & 0 & 0.00 & 2 & 0.67 \\
3-level 2D-DWT & 8306 & 10.14 & 9067 & 11.07 & 672 & 2.66 & 30 & 10.07 \\
Coeff. Grouping & 5960 & 7.28 & 6119 & 7.47 & 268 & 1.06 & 79 & 26.51 \\
BitPlaneEncoder & 8205 & 10.02 & 12157 & 14.84 & 395 & 1.56 & 11 & 3.69 \\
Output Muxer & 5035 & 6.15 & 10070 & 12.29 & 654 & 2.59 & 25 & 8.39 \\
\hline Total & 27630 & 33.73 & 37604 & 45.90 & 1989 & 7.87 & 147 & 49.33 \\
\hline
\end{tabular}

Table 5.5: Resource consumption of the architecture on a Virtex-5 XC5FX130T-1 for $d=24$ bit, $I_{h}=I_{w}=2048 \mathrm{px}$ and $S=128$ Blocks (Integer DWT only). The maximum frequency of the design on this particular FPGA is $125.33 \mathrm{MHz}$.

\begin{tabular}{lrrrrrrrr}
\hline Module & \multicolumn{2}{c}{ Slice Reg. } & \multicolumn{2}{c}{ LUT } & \multicolumn{3}{c}{ LUTRAM } & \multicolumn{2}{c}{ BlockRAM } \\
& abs. & $\%$ & abs. & $\%$ & abs. & $\%$ & abs. & $\%$ \\
\hline Compr. Control & 124 & 0.15 & 182 & 0.22 & 0 & 0.00 & 2 & 0.67 \\
3-level 2D-DWT & 8329 & 10.17 & 9041 & 11.04 & 672 & 2.66 & 34 & 11.41 \\
Coeff. Grouping & 5859 & 7.15 & 5724 & 6.99 & 268 & 1.06 & 100 & 33.56 \\
BitPlaneEncoder & 8216 & 10.03 & 12097 & 14.77 & 395 & 1.56 & 12 & 4.03 \\
Output Muxer & 5095 & 6.22 & 9852 & 12.03 & 654 & 2.59 & 25 & 8.39 \\
\hline Total & 27623 & 33.72 & 36896 & 45.04 & 1989 & 7.87 & 173 & 58.05 \\
\hline
\end{tabular}

\subsubsection{Kintex-7 Synthesis Results}

The resource consumption for the Kintex-7 XC7K325T-2 is shown in Table 5.8. Since this FPGA contains significantly more resources, the relative utilization is correspondingly lower. The DSP cores used for FP operations are faster so that the design can operate at $100 \mathrm{MHz}$ even the FP module is enabled. The number of BlockRAMs is higher so that the design can be synthesized for a maximum image width of $I_{w}=8192 \mathrm{px}$. The DWT module consumes much more resources when the FP DWT is also enabled. Beside DSP cores, slice registers, LUTs, and LUTRAMs are needed for each of the FP operations (see Figure 4.8). 
Table 5.6: Resource consumption of the architecture on a Virtex-5 XC5FX130T-1 for $d=24$ bit, $I_{h}=I_{w}=4096 \mathbf{p x}$ and $S=128$ Blocks (Integer DWT only). The maximum frequency of the design on this particular FPGA is $125.33 \mathrm{MHz}$.

\begin{tabular}{lrrrrrrrr}
\hline Module & \multicolumn{2}{c}{ Slice Reg. } & \multicolumn{2}{c}{ LUT } & \multicolumn{3}{c}{ LUTRAM } & \multicolumn{2}{c}{ BlockRAM } \\
& abs. & $\%$ & abs. & $\%$ & abs. & $\%$ & abs. & $\%$ \\
\hline Compr. Control & 124 & 0.15 & 176 & 0.21 & 0 & 0.00 & 2 & 0.67 \\
3-level 2D-DWT & 8349 & 10.19 & 9036 & 11.03 & 670 & 2.65 & 45 & 15.10 \\
Coeff. Grouping & 6377 & 7.78 & 5934 & 7.24 & 268 & 1.06 & 145 & 48.66 \\
BitPlaneEncoder & 8215 & 10.03 & 12085 & 14.75 & 395 & 1.56 & 11 & 3.69 \\
Output Muxer & 5027 & 6.14 & 10048 & 12.27 & 654 & 2.59 & 25 & 8.39 \\
\hline Total & 28092 & 34.29 & 37279 & 45.51 & 1987 & 7.86 & 228 & 76.51 \\
\hline
\end{tabular}

Table 5.7: Resource consumption of the architecture on a Virtex-5 XC5FX130T-1 for $d=24$ bit, $I_{h}=I_{w}=4096 \mathbf{p x}$ and $S=128$ Blocks (FP and Integer DWT). The maximum frequency of the design on this particular FPGA is $55.64 \mathrm{MHz}$.

\begin{tabular}{lrrrrrrrr}
\hline Module & \multicolumn{2}{c}{ Slice Reg. } & \multicolumn{2}{c}{ LUT } & \multicolumn{3}{c}{ LUTRAM } & \multicolumn{2}{c}{ BlockRAM } \\
& abs. & $\%$ & abs. & $\%$ & abs. & $\%$ & abs. & $\%$ \\
\hline Compr. Control & 135 & 0.16 & 190 & 0.23 & 0 & 0.00 & 2 & 0.67 \\
3-level 2D-DWT & 18425 & 22.49 & 39461 & 48.17 & 2431 & 9.62 & 43 & 14.43 \\
Coeff. Grouping & 6426 & 7.84 & 6028 & 7.36 & 268 & 1.06 & 143 & 47.99 \\
BitPlaneEncoder & 8036 & 9.81 & 12006 & 14.66 & 395 & 1.56 & 12 & 4.03 \\
Output Muxer & 5018 & 6.13 & 10024 & 12.24 & 654 & 2.59 & 26 & 8.72 \\
\hline Total & 38040 & 46.44 & 67709 & 82.65 & 3748 & 14.83 & 226 & 75.84 \\
\hline
\end{tabular}

\subsection{Power Consumption}

The knowledge about power consumption of the compression core is essential to estimate the total power consumption of a higher level component such as an FPA. In order to compare the results with other approaches, the total power consumption can be normalized with the data compression throughput. Since the whole compression core is implemented inside a single Xilinx FPGA, the power consumption can be reliably estimated by the Xilinx Power Estimator (XPE) [130]. Beside the FPGA device type, XPE needs the clock frequency of the design, the output load, parameters of the environment (for junction temperature estimation), the used resources of the FPGA design, toggle and enable rates of the logic, BlockRAMs, DSPs and I/O cells. The toggle and enable rates are set to their default values. These rates describe how often the output changes with respect to the input clock. It is usually between $6 \%$ and $12 \%$ for "a typical module for estimation purposes" [129]. For this investigation, the clock frequency of the design is set to the maximum clock frequency of the design $(100 \mathrm{MHz})$. The output load depends on the 
Table 5.8: Resource consumption of the architecture on a Kintex-7 XC7K325T-2 for $d=24$ bit, $I_{h}=I_{w}=4096 \mathrm{px}$ and $S=128$ Blocks (FP and Integer DWT). The maximum frequency of the design on this particular FPGA is $100.26 \mathrm{MHz}$.

\begin{tabular}{lrrrrrrrr}
\hline \multirow{2}{*}{ Module } & \multicolumn{2}{c}{ Slice Reg. } & \multicolumn{2}{c}{ LUT } & \multicolumn{3}{c}{ LUTRAM } & \multicolumn{2}{c}{ BlockRAM } \\
& abs. & $\%$ & abs. & $\%$ & abs. & $\%$ & abs. & $\%$ \\
\hline Compr. Control & 118 & 0.03 & 208 & 0.10 & 0 & 0.00 & 2 & 0.45 \\
3-level 2D-DWT & 15746 & 3.86 & 34293 & 16.83 & 2075 & 3.24 & 35 & 7.87 \\
Coeff. Grouping & 3337 & 0.82 & 3444 & 1.69 & 220 & 0.34 & 80 & 17.98 \\
BitPlaneEncoder & 8199 & 2.01 & 11511 & 5.65 & 405 & 0.63 & 13 & 2.92 \\
Output Muxer & 4933 & 1.21 & 10174 & 4.99 & 666 & 1.04 & 28 & 6.29 \\
\hline Total & 32333 & 7.93 & 59630 & 29.26 & 3366 & 5.26 & 158 & 35.51 \\
\hline
\end{tabular}

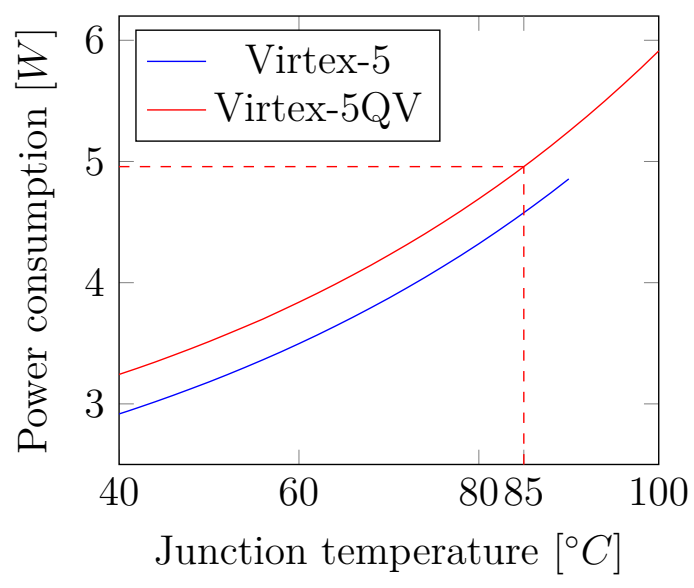

(a) Integer DWT only, $100 \mathrm{MHz}$.

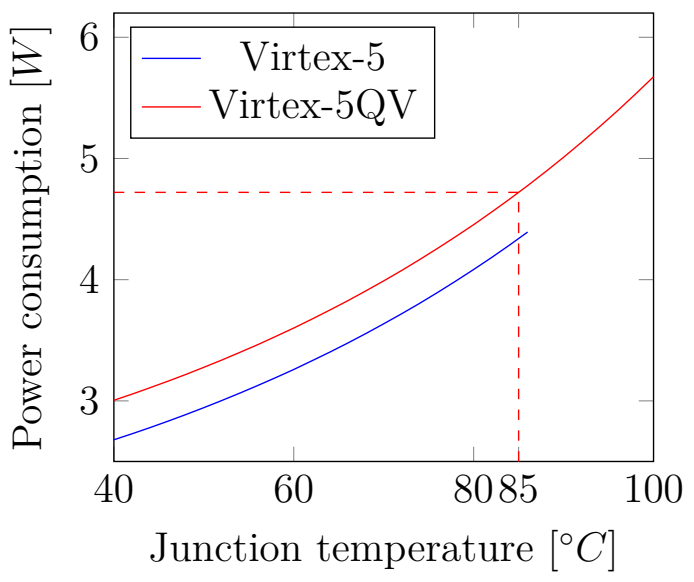

(b) Integer and FP DWT, $56 \mathrm{MHz}$.

Figure 5.2: Power consumption of the design on a Xilinx Virtex-5 and Virtex-5QV FPGA.

devices or signals driven by the FPGA. A value of $5 \mathrm{pF}$ is a typical value (default in XPE).

Figure 5.2 shows the power consumption of the design on a Xilinx Virtex-5 and Virtex$5 \mathrm{QV}$ FPGA. The relative power consumption per Mpx/s of the "Integer only" version is approximately $25 \mathrm{~mW} / \mathrm{Mpx} / \mathrm{s}$ at a frequency of $100 \mathrm{MHz}$ (total $4.96 \mathrm{~W}$ at $T_{j}=85^{\circ} \mathrm{C}$ junction temperature ${ }^{1}$ ). The total power consumption of the Integer and $\mathrm{FP}$ version is approximately $42 \mathrm{~mW} / \mathrm{Mpx} / \mathrm{s}$ at a frequency of $56 \mathrm{MHz}$ (total $4.72 \mathrm{~W}$ at $T_{j}=85^{\circ} \mathrm{C}$ ). The value of $T_{j}=85^{\circ} \mathrm{C}$ was chosen because it is a typical value according to our experience also for space applications and offers the possibility to compare the Virtex-5 and the Virtex-5QV. According to [131], "Xilinx recommends analyzing static power consumption at $T_{j}=85^{\circ} \mathrm{C}$ because the majority of designs operate near the high end of the commercial temperature range". The relative power consumption of $25 \mathrm{~mW} / \mathrm{Mpx} / \mathrm{s}$ is very low compared to other architectures (see following section).

\footnotetext{
${ }^{1}$ highest operation temperature of the semiconductor
} 


\subsection{Comparison with Other Implementations}

There are several implementations of the CCSDS 122.0-B-1 standard presented in literature. Software implementations are available at [118] and [44]. For this thesis, another software implementation including all extensions was developed for test and verification purposes. Initial tests show that a simple CPU implementation cannot fulfill the requirements on data throughput. In order to assess the implementation much better, a GPGPU implementation was developed by a student in collaboration with the author of this thesis [89]. The GPGPU architecture was chosen because it can potentially deliver a higher data throughput. The CPU and GPGPU platform hardware was mentioned in Section 5.1. The CPU was configured to operate on one processing core in order to obtain a near-sequential computation benchmark.

Table 5.9 lists the execution times and compression throughput for the images coastal_b4, ice_2kb4, foc, and sar of pixel depths 8, 10, and 16 bits per pixel respectively. The GPGPU implementation is very slow compared with both the CPU as well as the FPGA implementation. This is caused by the fact that (only) the outer segment-loop of the standard was parallelized and the number of segments is relatively low. A more finergrained, segment-based parallelization might be possible. The performance gap between the CPU and the GPGPU implementation becomes smaller with a rising segment size. It is to be assumed that GPGPU implementation will outperform the CPU at larger segment sizes.

Table 5.9: Comparison of execution times and data throughput for original images in Appendix B [89].

\begin{tabular}{lccccccr}
\hline \multirow{2}{*}{ Image } & \multirow{2}{*}{ \# Segments } & \multicolumn{3}{c}{ Execution time $(\mathbf{m s})$} & \multicolumn{3}{c}{ Throughput (Mbyte/s) } \\
\cline { 2 - 8 } & & CPU & GPU & FPGA & CPU & GPU & FPGA \\
\hline sar (16 bit) & 256 & 87.56 & 897.24 & 1.42 & 5.987 & 0.584 & 368.16 \\
foc (8 bit) & 512 & 83.69 & 404.58 & 2.72 & 9.397 & 1.944 & 192.98 \\
coastal_b4 (8 bit) & 1024 & 134.06 & 471.19 & 5.39 & 7.822 & 2.225 & 194.61 \\
ice_2kb4 (10 bit) & 4096 & 734.56 & 643.69 & 21.29 & 7.137 & 8.145 & 246.50 \\
\hline
\end{tabular}

In order to mitigate the low number of segments, measurements were repeated with larger images, e.g. derived by concatenation of the CCSDS reference images. The results are shown in Table 5.10. For segment sizes $S>=8192$, the GPGPU is approximately 10 times faster than the CPU implementation. The compression execution time of the FPGA implementation was not measured, but by analysis it should be approximately 16 times higher. Since all compression parameters except the number of segments are identical to the non-concatenated experiments, the data throughput should be nearby identical to the non-concatenated images. Further details and results of the GPGPU implementation are presented in [89]. 
Table 5.10: Comparison of execution times and throughput for 16 times concatenated image-set in Appendix B [89].

\begin{tabular}{lccccccr}
\hline \multirow{2}{*}{ Image } & \multirow{2}{*}{ \# Segments } & \multicolumn{3}{c}{ Execution time } & $(\mathbf{m s})$ & \multicolumn{3}{c}{ Throughput } & $(\mathrm{Mbyte} / \mathrm{s})$ \\
\cline { 2 - 7 } & & CPU & GPU & FPGA & CPU & GPU & FPGA \\
\hline sar (16 bit) & 4096 & 1289.41 & 243.40 & $\approx 23$ & 6.51 & 34.46 & $\approx 368$ \\
foc (8 bit) & 8192 & 1315.21 & 146.88 & $\approx 44$ & 9.57 & 85.67 & $\approx 193$ \\
coastal_b4 (8bit) & 16384 & 2502.23 & 257.79 & $\approx 86$ & 6.71 & 65.08 & $\approx 195$ \\
ice_2kb4 (10 bit) & 65536 & 12630.40 & 1316.13 & $\approx 341$ & 6.64 & 63.74 & $\approx 247$ \\
\hline
\end{tabular}

Hardware implementations of the CCSDS 122.0-B-1 compression standard have been presented in $[34,70,72,126]$. Table 5.11 shows some comparisons of these implementations. The table also mentions the Analog Devices ADV212 - a state-of-the-art JPEG2000 image encoder/decoder [10] that is not suitable for space applications. All implementations are limited in the image width (number of pixels in horizontal direction). For the other space implementations, the typical input data rate is between $15 \mathrm{Mpx} / \mathrm{s}$ and $60 \mathrm{Mpx} / \mathrm{s}$. For these implementations, there is no reliable information about the maximum image height. The approach presented in this thesis allows continuously image compression and thus, theoretically, it can compress images with an unlimited image height. The maximum image width without external memory is $4096 \mathrm{px}$ and with external memory it is limited by its size. Furthermore, the approach presented in this thesis can process an input data rate of up to $200 \mathrm{Mpx} / \mathrm{s}$ at the lowest relative power consumption per Mpx/s. A high input dynamic range of at least 12 bit is supported by all implementations.

Table 5.11: Comparison of image data compression implementations.

\begin{tabular}{|c|c|c|c|c|c|c|}
\hline & ADV212 & Winterrowd & Lin & $\mathbf{L i}$ & Airbus DS & This work \\
\hline Algorithm & JPEG2000 & CCSDS 122.0 & CCSDS 122.0 & CCSDS 122.0 & CCSDS 122.0 & CCSDS 122.0 \\
\hline Architecture & ASIC & RHBD ASIC & RHBD FPGA & rad. tol. FPGA & RHBD ASIC & RHBD FPGA \\
\hline Technology & COTS & $0.25 \mu \mathrm{m}$ CMOS & Virtex-5QV & RTAX2000S & ATC18RHA & Virtex-5QV \\
\hline Image width & 4096 & 8192 & 12000 & 5200 & 3496 & unlimited $^{2}$ \\
\hline Image height & 4096 & $?$ & unlimited? & unlimited? & unlimited? & unlimited \\
\hline Input dynamic range & 16 bit & 16 bit & 12 bit & 14 bit & 16 bit & 16 bit \\
\hline $\begin{array}{l}\text { Input data rate } \\
{[\mathrm{Mpx} / \mathrm{s}]}\end{array}$ & $65 \mathrm{Mpx} / \mathrm{s}$ & $20 \mathrm{Mpx} / \mathrm{s}$ & $40.4^{\mathrm{Mpx} / \mathrm{s}}$ & $\approx 15 \mathrm{Mpx} / \mathrm{s}$ & $60 \mathrm{Mpx} / \mathrm{s}$ & $200 \mathrm{Mpx} / \mathrm{s}$ \\
\hline Radiation (Total Dose) & - & $\geq 50 \mathrm{kRad}$ & $700 \mathrm{kRad}$ & $200 \mathrm{kRad}$ & $100 \mathrm{kRad}$ & $700 \mathrm{kRad}$ \\
\hline $\begin{array}{l}\text { Power consumption } \\
{[\mathrm{W} /(\mathrm{Mpx} / \mathrm{s})]}\end{array}$ & 0.05 & 0.17 & 0.06 & $\approx 0.03$ & 0.10 & 0.025 \\
\hline Reference & {$[10]$} & {$[126]$} & {$[71]$} & {$[70]$} & {$[34]$} & \\
\hline
\end{tabular}

A software implementation of the algorithm on an Aeroflex UT699 LEON3-FT ${ }^{3}$ is presented in [30]. According to [70], this implementation achieves a data compression

\footnotetext{
${ }^{2}$ The maximum line size (or width of the image) only depends on the size of the external memory.

${ }^{3}$ The Aeroflex UT699 LEON3-FT is a radiation hardened processor based on the Sparc ${ }^{\mathrm{TM}}$ V8 architecture [6].
} 
throughput of $0.63 \mathrm{Mbit} / \mathrm{s}$. For comparison, the software implementation of the CCSDS 122.0-B-1 algorithm created for this thesis reaches a throughput of $35 \mathrm{Mbit} / \mathrm{s}$ on a $3.2 \mathrm{GHz}$ Xeon processor (one thread). This difference can be explained by the fact, that the UT699 is a single-core processor running at $66 \mathrm{MHz}$. Aeroflex now also offers the GR740 quad-core processor running at up to $250 \mathrm{MHz}$ that should (neglecting architectural optimizations) theoretically be approximately 16 times faster [6].

\subsection{Impact of Lossy Image Compression}

In order to get a better understanding of the impact of lossy or near-lossless image compressing using the CCSDS 122.0-B-1 standard, several investigations were performed with remote sensing images, noise images, and noisy images. Since the application is possibly crucial to decide whether a certain amount of distortion is acceptable, a feature detection and matching algorithm as well as a stereo matching algorithm was used to show that even lossy compression might be acceptable. To determine the MTF (see Section 2.1.1.2), tools and test charts from [17] were used. Results of these investigations were presented in [75]. In this section, the results for the feature and stereo matching algorithm as well as the MTF investigations are shown.

\subsubsection{Compression and Feature Matching}

Features are often used to match a current image against another image (or images) to find corresponding image points. With these correspondences it might be possible to detect and track objects or to reconstruct the stereo information between two cameras. It is conceivable that image features are matched against features derived from compressed images. Due to the fact that both image compression and feature detection are non-linear, the influence of image compression on feature detection cannot be foretold. In order to investigate the impact of image compression on feature matching, scenes from the Leuven [69] database were used. It contains eight scenes each including 6 images and 5 transformation matrices.

For each test scene, scale-invariant feature transform (SIFT) features were calculated based on the first image of the dataset [73]. Then, images $2-6$ were compressed by CCSDS 122.0-B-1 at different compression ratios. After decompression, again SIFT features were calculated and matched against the features from the first image. With the transform matrix it is possible to decide whether a matching is good or bad: For each matching, the point in the first image is transformed to the position in the second image. Then, the distance from this position to the position of the matching feature was calculated. If the distance is higher than a threshold of $10 \mathrm{px}$, the matching was classified as bad. This threshold value tolerates little deviations, e.g. caused by different viewing angle or image noise. 


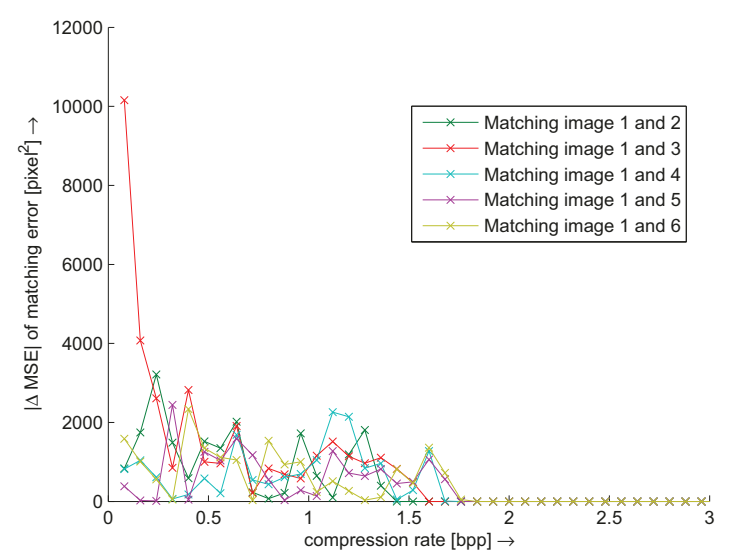

(a) image bark

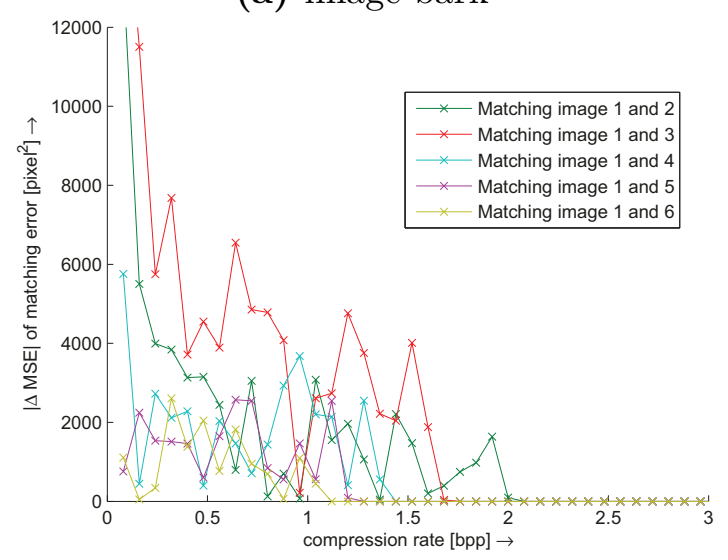

(c) image trees

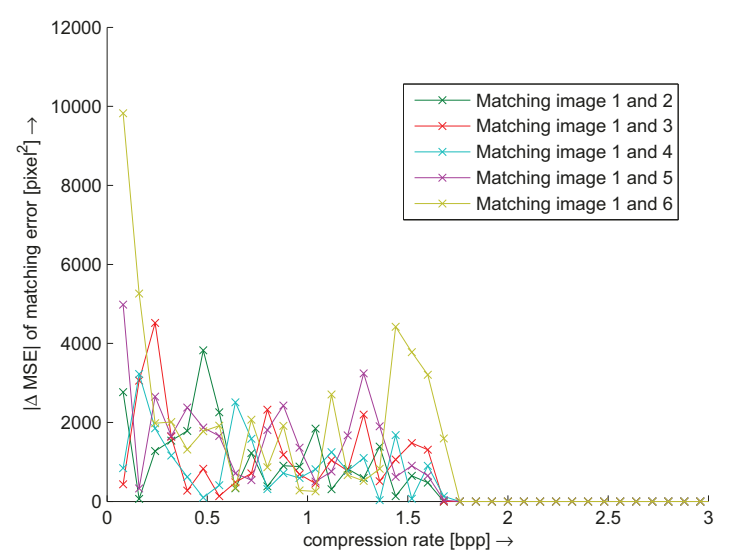

(b) image graf

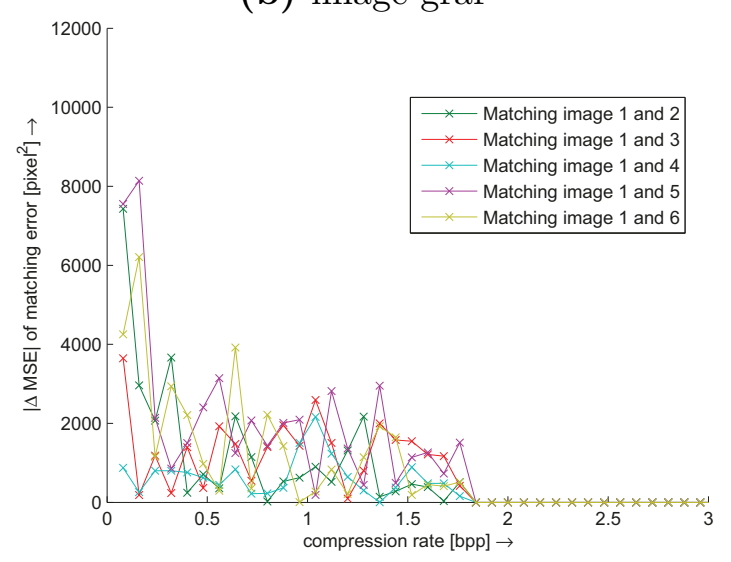

(d) image wall

Figure 5.3: Impact of image compression on feature matching.

For the remaining (good) matching, the mean-squared error $\operatorname{MSE}(r)$ of the matched and real positions is determined in as a function of the compression rate. Now, the absolute value of the difference between the error without or under lossless compression $\mathrm{MSE}_{0}$ and the actual error $\operatorname{MSE}(r)$ is determined:

$$
\Delta \operatorname{MSE}(r)=\left|\mathrm{MSE}_{0}-\operatorname{MSE}(r)\right|
$$

Figure 5.3 shows the the value of $\Delta \mathrm{MSE}(r)$ for the scenes bark, graf, trees and wall. For a bit rate of less than $2 \mathrm{bpp}$, there are high values for $\triangle \mathrm{MSE}(r)$ for all scenes. At a bit rate higher than $2 \mathrm{bpp}$, the errors according to $\mathrm{MSE}_{0}$ become zero, which means that feature detection and matching were not influenced by compression.

\subsubsection{Compression and Stereo-Matching}

As image quality highly depends on the application in which the images are used, a stereo-matching algorithm was chosen for another test. Beside feature matching, stereomatching algorithms are used more and more in the interpretation of remote sensing 
images. The datasets from the Middlebury Stereo Vision Page [99] and the StereoSGBM matching implementation in OpenCV [14] were used. The database contains four image pairs (cones, teddy, tsukuba, and venus), the corresponding stereo ground truth depthor disparity map, and three masks which are needed for stereo-matching evaluation. For these tests, the mask "all" was chosen containing all pixels including half occluded pixels. To measure the matching error, for all pixels, where the corresponding value in the mask is 255, the absolute value of the difference of the calculated disparity and the ground truth was calculated. Then, the number of pixels, where this value is larger than 1 is multiplied by 100 and divided by the number of pixels selected by the mask. So this is the percentage of faulty pixels in the disparity map (see [99] for more details).

Figure 5.4 shows the matching error as a function of the compression bit-rate for the 4 images of the Middlebury Stereo dataset [99]. The matching error of the uncompressed image is equal to the matching at the right end of the curves (corresponds lossless compression). One can see that at around $4 \mathrm{bpp}$, there is almost no error for all images. The matching error rises at around 2-2.5 bpp. At $1 \mathrm{bpp}$, the matching error around $5 \%$ higher than without compression. At $0.5 \mathrm{bpp}$, it is around as twice as high as without compression. Even though these tests are very rudimentary and probably not representative, they give a hint that lossy compression at a compression ratio of approximately 4:1 can be possible for image data to be used for stereo-matching. It is conceivable that high-resolution stereo images are compressed on-board the spacecraft for further ground processing.

\subsubsection{Impact of Compression on Image MTF}

To determine the MTF, tools and test charts from [17] and the image "boat" were used. The tool sfrmat3 uses the slanted edge method [63]. Two images were chosen for evaluation. ROIs for MTF determination were selected for both images. In the image "ISO_DSC_300_mono", the area is in $x=[515,731]$ and $y=[337,454]$ and for the image "boat", the area is in $x=[337,378]$ and $y=[255,279]$. The compression ratios were $0.5 \mathrm{bpp}$ and $1 \mathrm{bpp}$ for the test chart, and $1 \mathrm{bpp}$ and $2 \mathrm{bpp}$ for the image "boat". Figure 5.5 shows the MTF before compression and after decompression on the same region of interest.

One can see that for $1 \mathrm{bpp}$, the MTF of the test chart, and for $2 \mathrm{bpp}$, the MTF of the image "boat" are nearly unaffected. When increasing the compression ratios, both MTF curves degrade. But because of the non-linearity of the image compression, results must be interpreted with caution. It may happen that the compression introduces high frequency distortions (like the typical JPEG artifacts) that erroneously improves the MTF. The dependence of the MTF and compression rate was also investigated for other bit rates and the MTF curves show, that the MTF is stable at higher bit rates than 2 bpp for the image "boat" and 1 bpp for the test chart. 


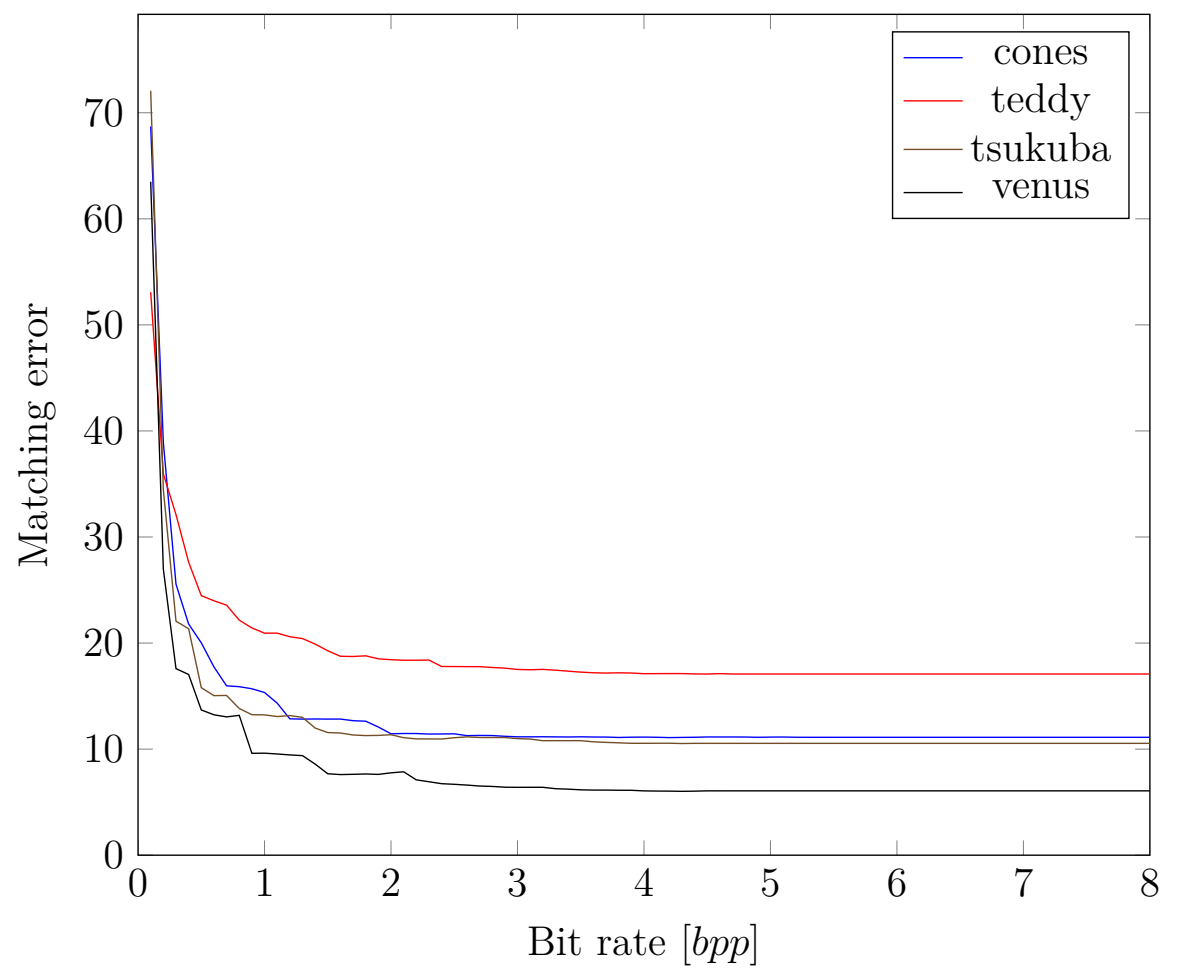

Figure 5.4: Impact of image compression on stereo-matching. The figure shows the stereo matching error as a function of the compression bit-rate for the 4 images of the Middlebury Stereo dataset [99].

\subsection{Summary}

This chapter has presented and discussed the experimental results of this thesis. It has been shown that all of the requirements presented in Chapter 4 were met. The architecture presented in this thesis combines both possibilities for pre- and post-compression ROI support. The results for the resource and power consumption show that it is possible to implement the image compression onto an FPA using an FPGA. As far as known, the architecture enables the fastest implementation of the CCSDS 122.0-B-1 standard yet reported. The impact of lossy image compression on image quality was investigated on selected examples. The results show that a compression ratio of $1: 4$ (2 bpp bit rate for 8 bit images) has almost no impact on the quality of the results. 


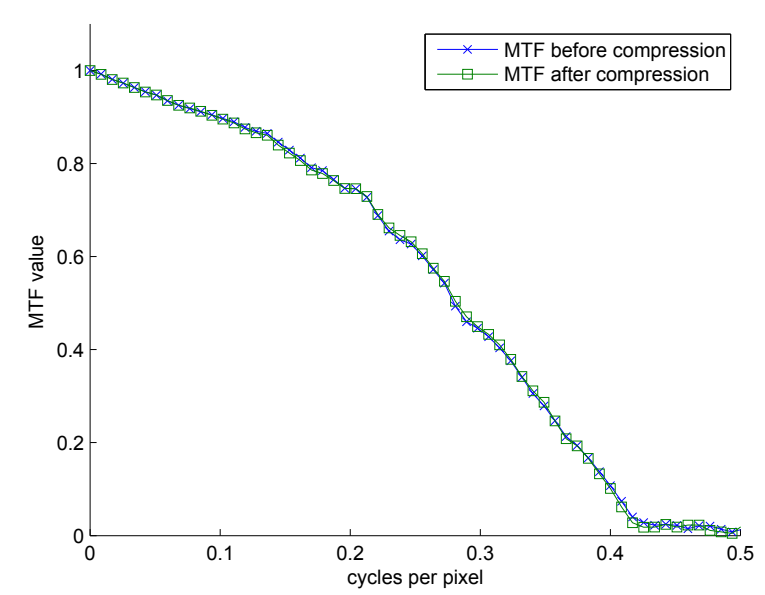

(a) test chart 1 bpp

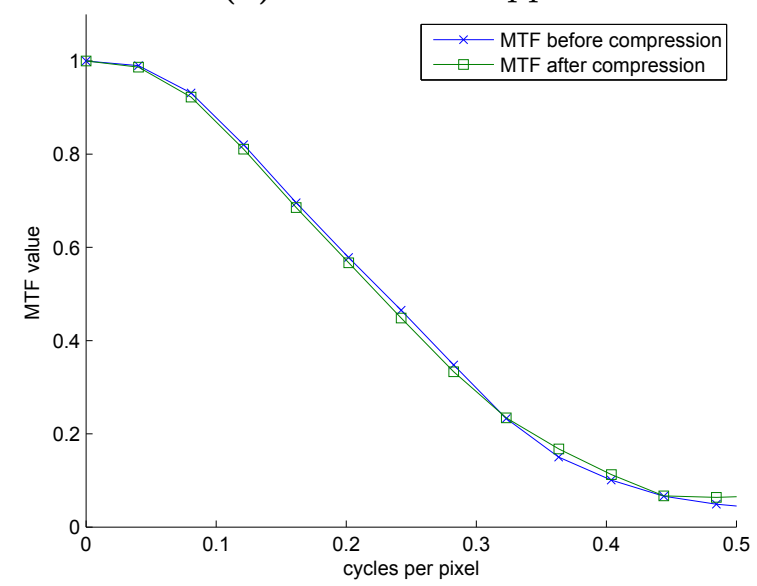

(c) image "boat" 2 bpp

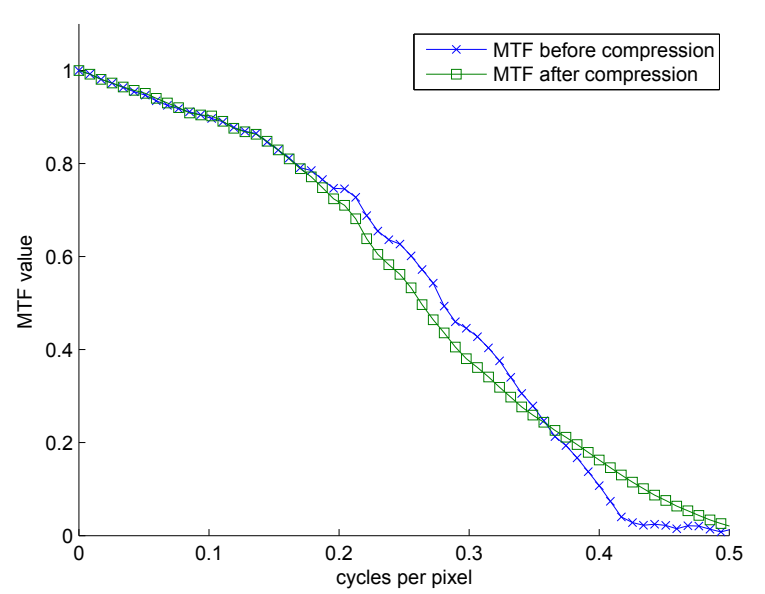

(b) test chart $0.5 \mathrm{bpp}$

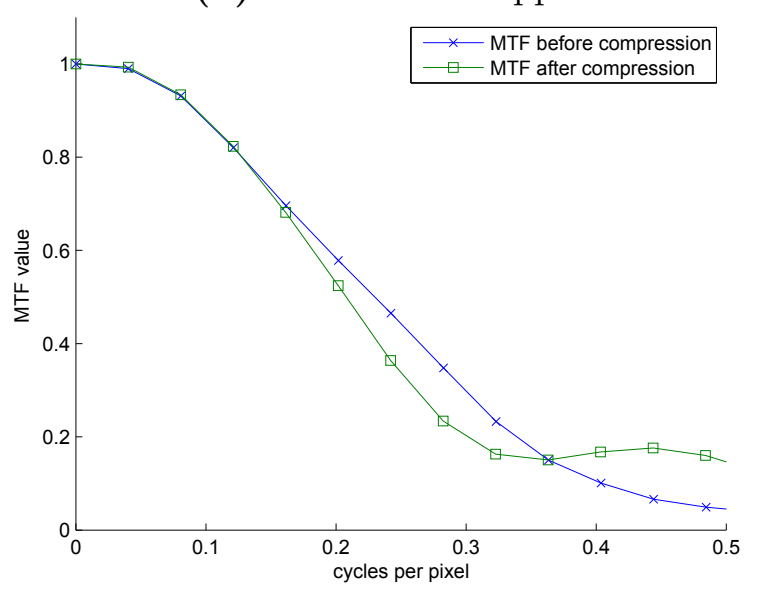

(d) image "boat" 1 bpp

Figure 5.5: Impact of image compression on the MTF. 


\section{Conclusions and Future Work}

In this thesis, first the fundamentals of optical satellite imagery, image data compression on-board a spacecraft, and the CCSDS 122.0-B-1 image data compression standard have been presented. Thereafter, extensions to the CCSDS 122.0-B-1 standard and the design and implementation of a new image data compression architecture has been developed and verified. The extensions allow multi-spectral compression, ROI coding, and re-assembling of the compressed image or parts of it to achieve a particular image quality, spatial or spectral resolution in any area without re-coding. This thesis presents the fastest implementation of the CCSDS 122.0-B-1 standard yet reported. The extensions proposed in Chapter 3, the architecture described in Chapter 4, and the experimental results shown in Chapter 5 have partly been published in [75-77, 89].

The following key points characterize the new architecture:

- It implements the CCSDS 122.0-B-1 image data compression standard.

- It uses an optimized stream-pipeline structure instead of increasing the number of compression cores.

- It can process up to two pixels per clock cycle.

- It can compress images with arbitrary width (limited only by memory) and height.

- It can be applied for multi-spectral compression (Section 3.3).

- It support ROI coding as presented in Section 3.1.

- It supports scalability as presented in Section 3.2.

- It can be implemented on space-qualified technology (Virtex-5QV or Microsemi RTG4).

- It is scalable to high clock frequencies.

The proposed data compression architecture can be used wherever a large amount of image data must be compressed in a short time. However, it has some restrictions and room for additional optimizations. During the creation of this thesis and related studies, a number of issues and ideas have arisen that are presented in this chapter. 


\subsection{Advanced Hardware Demonstrator}

In order to prove the functionality of the approach even for large images requiring external memory, an evaluation platform based on a COTS FPGA board is currently being developed. Since the approach needs high-bandwidth and low-latency external memory, the HiTechGlobal NetFPGA10G FPGA board was selected [48]. It contains a Xilinx Virtex-5 XC5VTX240T FPGA, three 72Mbit QDRII+ SRAM modules and some other interfaces. Since all important parts are available in a radiation hardened, space qualified version, this platform is ideal for evaluation. The board was integrated in the hardware operating system presented in Section 2.3.3. An integration of the QDRII+ SRAM is currently being developed. Since the compression architecture is adaptable to other mission requirements, the evaluation platform might also be used for other and future projects.

The hardware demonstrator shall also include an algorithm for ROI extraction as well as the functionality of the bit-stream re-assembling process.

\subsection{Integrated Focal Plane}

A high resolution and data-rate FPA with integrated image data compression has not yet been developed at the DLR. To the best of our knowledge this does not yet exist anywhere. All missions using image data compression use a separate module for image data compression (most often something like a data compression and storage unit).

The impact of image data compression on the FPA regarding FPGA resources and power consumption was investigated in this thesis. These preliminary investigations are necessary in order to estimate the feasibility to integrate image data compression into a future FPA. Since the Virtex-5 FPGA is now available for space applications, the major problem of FPGA resources can be considered as solved. It can be assumed that the architecture presented in this thesis can be used for a future FPA providing the necessary resources, since it can be very well adapted to special requirements.

\subsection{Further Extensions for the Compression Algorithm}

The number of DWT levels can be increased in order to improve spatial decorrelation and compression efficiency. JPEG2000 uses a five-level two-dimensional DWT. An extension of CCSDS 122.0-B-1 that also uses a five-level transform was presented in [41]. However, this approach would significantly increase the amount of memory that is needed for wavelet transform and entropy coding, since a block will consist of $\left(2^{5}\right)^{2}=32^{2}=1024$ coefficients. Beside a more complex structure in terms of control logic, the amount of memory used to group coefficients to segments and to buffer these segments for coding will be approximately 16 times higher. Extending the proposed highly parallel architecture for a higher number of wavelet decomposition levels is regarded as not feasible with the 
available FPGA technology.

For multi-spectral compression, a well-chosen spectral decorrelation method that offers a trade-off between implementation complexity and compression efficiency would be useful. KLT spectral decorrelation offers the highest degree of decorrelation, but on the other hand it has a high implementation complexity. As mentioned in Section 3.3 a static transform matrix derived from a set of training images can solve this issue. The implementation of the decorrelation is much easier then, since only the transform must be applied.

From the engineering point of view, it is desirable to estimate the impact of image compression on the MTF and SNR, since these parameters are used to characterize the image quality of an imaging system. Online image quality assessment might be used to control the compression parameters to ensure the image quality will not fall under a certain threshold. In view of the algorithm, it would be conceivable to terminate or stop the coding process, if a certain quality is reached. An image compression adviser that analyzes statistical features of the image in order to determine whether lossless or lossy compression should be used was presented in [45].

\subsection{Improvements for the Compression Architecture}

Several ideas regarding the compression architecture have emerged, which have not yet been implemented. Since the DWT modules in the higher decomposition levels are not fully utilized, it is possible to multiplex the data streams and to share a single DWT module between the decomposition stages. Since the Float Point DWT has a high resource consumption, it is planned to implement a fixed-point version of the DWT. The precision of the transform can be influenced by the number of decimal points. A total number of 24 bits to represent the fixed-point numbers should be enough, whereas the scaling factor may be different depending on the decomposition stage [38].

A GPU implementation of the compression algorithm has also been developed. As long as there are no radiation tolerant GPUs available, it can only be used for airborne applications.

\subsection{Towards Further On-Board Processing}

Since the download data rate is usually much lower than the image acquisition rate, on-board re-assembling of the bit-stream should be feasible using software running on a CPU. It is conceivable to use a processor such as the Freescale P4080, which has 8 embedded PowerPC cores running at $1.5 \mathrm{GHz}$. A radiation tolerant version of the Freescale P4080 is available from e2v [100]. 
It is conceivable that a relatively "low-cost satellite" with less or without mass storage and a continuous low-speed data connection sends image data immediately to a (mobile) ground station. The satellite buffers, for example, image data for only a few minutes. Due to bandwidth limitations, only the most interesting images or image parts can be sent. A region-of-interest is programmed by the ground station or determined based on the actual image data. Motion detection might be realized with a second (redundant) detector that acquires the same scene from a slightly different viewing angle somewhat "earlier".

Interactive transmission can be realized with the concept of scalability presented in Section 3.2. On ground, it is conceivable that an image processing algorithm immediately operates on the received preview of an image during transmission. If an area or event is detected, a higher detail of the corresponding area might be required. The algorithm can request a higher resolution of this area without retransmitting the complete image. If the satellite has a limited downlink capability, interactive transmission and decoding are suitable methods, because there is usually not enough time for re-coding or re-transmission.

The usual motivation for using image compression on-board satellites is a limited storage or download capacity. It can be assumed that even future developments in radio transmission will most likely not allow a real-time transmission of an increasing amount of data. Beside the classic scenario of a store-and-download architecture, more advanced application scenarios are imaginable:

Change/Event detection: If the satellite imaging system acquires images over a certain area, it can compare the current image data with an older version stored on-board the satellite. This requires that a spatial mapping between both images is performed. Changes that are caused by natural disasters such as floods, earthquakes or storms, or large objects that have moved, could be detected. In conjunction with an image data compression, the detected areas can now be stored in a high quality, while the other areas are stored in low quality. Depending on whether online or offline image compression is used, the detection algorithms must also operate online or offline, respectively.

Object detection: The quality parameters of the image data compression algorithm can also be adapted to the image content. Object detection algorithms can be used to find objects of interest, e. g. objects with a certain shape, size or color. Clouds, for example, are usually not of interest and can be compressed in a low quality (or omitted) in the image acquisition process. [125]. Another example would be the verification of ship positions, similar as it is done in [104] in combination with radar images. 


\section{References}

[1] 3D PLUS SA. Memory Module, DDR2 SDRam 512Mx8-SOP. URL: http://www. 3d-plus.com/doc/prod/3dfp_0283_3.pdf (visited on 04/01/2014).

[2] 3D PLUS SA. Memory Module, DDR2 SDRam 64Mx72-BGA. URL: http://www. 3d-plus.com/doc/prod/3dfp_0478_3.pdf (visited on 04/01/2014).

[3] 3D PLUS SA. Memory Module, FLASH Nand 512Mx64-BGA. URL: http://www. 3d-plus.com/doc/prod/3dfp_0263_3.pdf (visited on 04/01/2014).

[4] G.P. Abousleman, M.W. Marcellin, and B.R. Hunt. "Compression of hyperspectral imagery using the 3-D DCT and hybrid DPCM/DCT". In: Geoscience and Remote Sensing, IEEE Transactions on 33.1 (Jan. 1995), pp. 26-34. ISSN: 0196-2892. DOI: $10.1109 / 36.368225$.

[5] Tinku Acharya and Chaitali Chakrabarti. "A Survey on Lifting-based Discrete Wavelet Transform Architectures". In: Journal of VLSI signal processing systems for signal, image and video technology 42.3 (2006), pp. 321-339. ISSN: 0922-5773. DOI: $10.1007 / \mathrm{s} 11266-006-4191-3$.

[6] Aeroflex. LEON 3FT Microprocessor. URL: http://ams.aeroflex.com/pagesproduct/ prods-hirel-leon.cfm (visited on 04/01/2014).

[7] Aeroflex UTMC Microelectronic Systems Inc. UT54LVDS217 Serializer. 2011. URL: http://www . aeroflex.com/ams/pagesproduct/datasheets/lvdsserializer. pdf (visited on 04/01/2014).

[8] Aeroflex UTMC Microelectronic Systems Inc. UT54LVDS218 Deserializer. 2011. URL: http://www . aeroflex.com/ams/pagesproduct/datasheets/lvdsdeserializer . pdf (visited on 04/01/2014).

[9] B. Aiazzi, P. Alba, L. Alparone, and S. Baronti. "Lossless compression of multi/hyperspectral imagery based on a 3-D fuzzy prediction". In: Geoscience and Remote Sensing, IEEE Transactions on 37.5 (Sept. 1999), pp. 2287-2294. ISSN: 0196-2892. DOI: $10.1109 / 36.789625$.

[10] Analog Devices. ADV212, Wavescale Video Codec. 2010. URL: http: / / www . analog.com/static/imported-files/data_sheets/ADV212.pdf (visited on 04/01/2014).

[11] Daniel Bachofen. FPGA Wavelet Transformation für Bildübertragung. Diplomarbeit. Hochschule für Technik, Wirtschaft und Soziale Arbeit St.Gallen, Fachbereich Elektrotechnik, Oct. 2001. 
[12] S. Benierbah and M. Khamadja. "Compression of colour images by inter-band compensated prediction". In: Vision, Image and Signal Processing, IEE Proceedings - 153.2 (Apr. 2006), pp. 237-243. ISSN: 1350-245X. DOI: 10.1049 / ip-vis : 20050129.

[13] Philippe Blanc and Lucien Wald. "A review of earth-viewing methods for inflight assessment of modulation transfer function and noise of optical spaceborne sensors". Working Paper. 2009. URL: http://hal . archives-ouvertes.fr/ docs/00/74/50/76/PDF/Working_Paper_MTF_SNR_blanc-wald.pdf (visited on 04/01/2014).

[14] G. Bradski. "The OpenCV Library". In: Dr. Dobb's Journal of Software Tools (2000).

[15] Fredrik Brosser and Emil Milh. "SEU Mitigation Techniques for Advanced Reprogrammable FPGA in Space". MA thesis. Chalmers University of Technology, Department of Computer Science and Engineering, 2014.

[16] Bernard V. Brower, Michael A. Cosgrove, Donald H. Lewis, Glenn R. VanLare, Thomas J. Fisher, and David M. Charneski. "Advanced space-qualified downlink image compression ASIC for commercial remote sensing applications". In: Proc. SPIE 4115 (2000), pp. 311-319. DOI: 10.1117/12.411555. URL: http://dx.doi. org/10.1117/12.411555.

[17] Peter D. Burns. sfrmat3: SFR analysis for digital cameras and scanners. 2009. URL: http://losburns. com/imaging/sof tware/SFRedge/ (visited on 04/01/2014).

[18] A. R. Calderbank, Ingrid Daubechies, Wim Sweldens, and Boon-lock Yeo. "Wavelet transforms that map integers to integers". In: Applied and Computational Harmonic Analysis 5.3 (1998), pp. 332-369. DOI: http://dx.doi.org/10.1006/ acha. 1997.0238.

[19] A.R. Calderbank, I. Daubechies, W. Sweldens, and Boon-Lock Yeo. "Lossless image compression using integer to integer wavelet transforms". In: Image Processing, 1997. Proceedings., International Conference on. Vol. 1. Oct. 1997, 596-599 vol.1. DOI: $10.1109 /$ ICIP. 1997.647983.

[20] Hongyang Chao, Paul Fisher, and Zeyi Hua. "An Approach to Integer Wavelet Transformations for Lossless Image Compression". In: in Proc. of International Symposium on Computational Mathematics. 1997, pp. 19-38.

[21] Liang-Gee Chen, Chao-Tsung Huang, Ching-Yeh Chen, and Chih-Chi Cheng. VLSI Design of Wavelet Transform, Analysis, Architecture, and Design Examples. London: Imperial College Press, Dec. 2006. ISBN: 1860946739.

[22] Charilaos Christopoulos, Athanassios Skodras, and Touradj Ebrahimi. "The JPEG2000 still image coding system: an overview". In: Consumer Electronics, IEEE Transactions on 46.4 (2000), pp. 1103-1127. ISSN: 0098-3063. DOI: $10.1109 / 30.920468$. 
[23] A. Cohen, Ingrid Daubechies, and J.-C. Feauveau. "Biorthogonal bases of compactly supported wavelets". In: Communications on Pure and Applied Mathematics 45.5 (1992), pp. 485-560. ISSN: 1097-0312. DOI: 10.1002/cpa.3160450502. URL: http://dx.doi.org/10.1002/cpa.3160450502.

[24] Consultative Committee for Space Data Systems (CCSDS). Image Data Compression - Blue Book. Recommendation for Space Data System Standards. Consultative Committee for Space Data Systems (CCSDS), 2005. uRL: http://public.ccsds . org/publications/archive/122x0b1c3.pdf (visited on 04/01/2014).

[25] Consultative Committee for Space Data Systems (CCSDS). Image Data Compression - Green Book. Report Concerning Space Data System Standards. Consultative Committee for Space Data Systems (CCSDS), 2007. URL: http://public.ccsds . org/publications/archive/120x1g1e1.pdf (visited on 04/01/2014).

[26] Consultative Committee for Space Data Systems (CCSDS). Space Link Services Area. Consultative Committee for Space Data Systems (CCSDS), 2007. URL: http: / / cwe . ccsds . org/sls / docs / Forms / Allitems . aspx ? RootFolder $=\% 2 \mathrm{fsls} \%$ $2 \mathrm{fdocs} \%$ 2fSLS $\% 2 \mathrm{dDC} \%$ 2fBB122TestData \& FolderCTID $=\& V i e w=\% 7 \mathrm{~b} 16 \mathrm{ACDA} 38 \%$ $2 \mathrm{dFFA3} \% 2 \mathrm{~d} 4657 \% 2 \mathrm{~d} 8 \mathrm{~F} 27 \% 2 \mathrm{~dB} 166 \mathrm{C} 23 \mathrm{C} 24 \mathrm{~A} 2 \% 7 \mathrm{~d}$ (visited on $04 / 01 / 2014$ ).

[27] Cypress Semiconductor Corporation. Radiation hardened products - QDR II+ SRAM. URL: http: //www . cypress . com/?docID=48207 (visited on 04/01/2014).

[28] A. S. Dawood, J. A. Williams, and S. J. Visser. "On-board satellite image compression using reconfigurable FPGAs". In: Field-Programmable Technology, 2002. (FPT). Proceedings. 2002 IEEE International Conference on. Dec. 2002, pp. 306310. DOI: 10.1109/FPT.2002.1188698.

[29] Jonathan Delcourt, Alamin Mansouri, Tadeusz Sliwa, and Yvon Voisin. "An Adaptive Multiresolution-Based Multispectral Image Compression Method". In: (2010).

[30] Anna Maria Di Giorgio, Paolo Bastia, Scige J. Liu, Giovanni Giusi, Roberto Scaramella, Mark Cropper, Richard Cole, Ady James, Jérôme Amiaux, and Yannick Mellier. The command and data processing unit of the EUCLID visible imager: impact of the data compression needs on the unit design. 2012. DOI: 10.1117/12.926638. URL: http://dx.doi.org/10.1117/12.926638.

[31] Mark S. Drew and Steven Bergner. "Spatio-chromatic decorrelation for color image compression". In: Image Commun. 23 (8 Sept. 2008), pp. 599-609. ISSN: 0923-5965. DOI: $10.1016 / \mathrm{j}$. image .2008.05.006. URL: http://dl .acm.org/ citation. cfm?id=1410884.1410900.

[32] Qian Du and J.E. Fowler. "Hyperspectral Image Compression Using JPEG2000 and Principal Component Analysis". In: Geoscience and Remote Sensing Letters, IEEE 4.2 (Apr. 2007), pp. 201-205. ISSN: 1545-598X. DOI: 10.1109/LGRS. 2006.888109.

[33] EADS Astrium. CORECI - An integrated COmpression REcording and CIphering solution for Earth Observation satellites. 2009. URL: http://www.astrium.eads . net/media/document/compression-memory-unit.pdf (visited on 04/01/2014). 
[34] EADS Astrium. CWICOM: Your Image Compression Core on a Single Chip. 2013. URL: http://spacewire.esa.int/edp-page/documents/CWICOM\%20Product\% 20Sheet.pdf (visited on 04/01/2014).

[35] eoPortal Directory. KOMPSAT-3 (Korea Multi-Purpose Satellite-3) / Arirang3. URL: https : / / directory . eoportal . org / web / eoportal / satellitemissions/k/kompsat-3 (visited on 04/01/2014).

[36] M. Fleury, R. P. Self, and A. C. Downton. "Multi-spectral satellite image processing on a platform FPGA engine". In: In Military and Aeronautics Logic Devices (MAPLD 05), 2005. Paper. 2005, p. 133.

[37] Joaquín Franco, Gregorio Bernabé, Juan Fernández, and Manuel E. Acacio. "A Parallel Implementation of the 2D Wavelet Transform Using CUDA". In: 2009 17th Euromicro International Conference on Parallel, Distributed and Network-based Processing. IEEE, 2009, pp. 111-118. ISBN: 978-0-7695-3544-9. DOI: 10.1109/PDP . 2009.40. URL: http://ieexplore.ieee.org/lpdocs/epic03/wrapper.htm? arnumber $=4912922$.

[38] T.W. Fry and S.A. Hauck. "SPIHT image compression on FPGAs". In: Circuits and Systems for Video Technology, IEEE Transactions on 15.9 (Sept. 2005), pp. 1138-1147. ISSN: 1051-8215. DOI: 10.1109/TCSVT.2005.852625.

[39] Jiri Gaisler. A structured VHDL design method. Oct. 1997. URL: http: //www . gaisler.com/doc/vhdl2proc.pdf (visited on 04/01/2014).

[40] D.D. Gajski and R.H. Kuhn. "Guest Editors' Introduction: New VLSI Tools". In: Computer 16.12 (Dec. 1983), pp. 11-14. ISSN: 0018-9162. DOI: 10.1109/MC.1983. 1654264.

[41] F. Garcia-Vilchez and J. Serra-Sagrista. "Extending the CCSDS Recommendation for Image Data Compression for Remote Sensing Scenarios". In: IEEE Transactions on Geoscience and Remote Sensing 47.10 (Oct. 2009), pp. 3431-3445. ISSN: 01962892. DOI: $10.1109 /$ TGRS . 2009.2021067.

[42] Fernando Garcia-Vilchez, Joan Serra-Sagrista, Joan Bartrina-Rapesta, and Francesc Auli-Llinas. "Hyperspectral Image Coding Using 3D Transform and the Recommendation CCSDS-122-B-1". In: Proceedings of the Data Compression Conference. Washington, DC, USA: IEEE Computer Society, 2008, pp. 103-112. ISBN: 978-07695-3121-2. DOI: 10.1109/DCC.2008.68. URL: http://dl.acm.org/citation. cfm?id=1395764. 1395971 .

[43] A. García, L. Santos, S. López, G. M. Callicó, J. F. López, and R. Sarmiento. "FPGA implementation of the hyperspectral Lossy Compression for Exomars (LCE) algorithm". In: SPIE Remote Sensing. Ed. by Bormin Huang, Sebastian López, and Zhensen Wu. International Society for Optics and Photonics, Nov. 2014, p. 924705. DOI: 10.1117/12.2069493. URL: http://proceedings . spiedigitallibrary. org/proceeding. aspx?articleid=1937651. 
[44] Group on Interactive Coding of Images (GICI) at the Universitat Autonoma de Barcelona (UAB). TER - An implementation of CCSDS Recommendation for Image Data Compression. 2008. URL: http://www.gici.uab.cat/TER/.

[45] A. Guzman and M. Beltran. "Satellite on-board image compression adviser". In: Signal Processing and Information Technology, 2004. Proceedings of the Fourth IEEE International Symposium on. Dec. 2004, pp. 296-301. DOI: 10.1109/ISSPIT . 2004.1433743.

[46] Ofer Hadar, Adrian Stern, Merav Huber, and Revital Huber. "Modulation transfer function as a quality measure for compressed images transmitted over a lossy packet network". In: Optical Engineering 40.10 (2001), pp. 2134-2142. DOI: 10. 1117/1.1397766. URL: http://dx.doi.org/10.1117/1.1397766.

[47] Heinz Haferkorn. Optik - Physikalische-technische Grundlagen und Anwendungen. John Wiley \& Sons, Inc., 2008. ISBN: 9783527625017. DOI: 10 . 1002 / 9783527625017.fmatter. URL: http://dx.doi.org/10.1002/9783527625017. fmatter.

[48] HiTech Global, LLC. HTG-540 : Xilinx Virtex5 TX240T PCI Express 8340 GIG SFP+ Development Platform - NetFPGA10G. 2016. URL: http: //www . hitechglobal.com/Boards/PCIExpress_SFP+.htm (visited on 07/01/2016).

[49] Chao-Tsung Huang, Po-Chih Tseng, and Liang-Gee Chen. "Flipping structure: an efficient VLSI architecture for lifting-based discrete wavelet transform". In: Circuits and Systems, 2002. APCCAS '02. 2002 Asia-Pacific Conference on. Vol. 1. 2002, 383-388 vol.1. DOI: 10.1109/APCCAS .2002.1114975.

[50] D.A. Huffman. "A Method for the Construction of Minimum-Redundancy Codes". In: Proceedings of the IRE 40.9 (Sept. 1952), pp. 1098-1101. ISSN: 0096-8390. DOI: 10.1109/JRPROC. 1952.273898.

[51] "IEEE Standard for Binary Floating-Point Arithmetic". In: ANSI/IEEE Std 7541985 (1985). DOI: 10.1109/IEEESTD. 1985.82928. URL: http://754r.ucbtest. org/standards/754.pdf.

[52] Asad Islam and William A. Pearlman. "Embedded and efficient low-complexity hierarchical image coder". In: Proc. SPIE 3653 (1998), pp. 294-305. DOI: 10. 1117/12.334677. URL: http://dx.doi.org/10.1117/12.334677.

[53] ITU. ISO/IEC 10918-1 : 1993(E) CCIT Recommendation T.81. 1993. URL: http: //www.w3.org/Graphics/JPEG/itu-t81.pdf (visited on 04/01/2014).

[54] ITU. JPEG 2000 image coding system: Core coding system, ISO/IEC 15444-1:2004 / ITU-T Rec. T.800. 2004. URL: http://www.iso.org/iso/catalogue_detail. htm?csnumber=37674 (visited on 04/01/2014).

[55] ITU. ITU Recommendation T.808 - JPIP. 2008. URL: http://www.itu.int/ rec/T-REC-T.808/en (visited on 04/01/2014). 
[56] Jet Propulsion Laboratory - California Institute of Technology. AVIRIS - Airborne Visible/Infrared Imaging Spectrometer. URL: http: //aviris.jpl . nasa.gov/ (visited on 04/01/2014).

[57] Jet Propulsion Laboratory - California Institute of Technology. AVIRIS Hyperspectral Images, Hyperspectral Image Compression. URL: http://compression. jpl.nasa.gov/hyperspectral/ (visited on 04/01/2014).

[58] A. Kaarna. "Integer PCA and wavelet transforms for multispectral image compression". In: Geoscience and Remote Sensing Symposium, 2001. IGARSS '01. IEEE 2001 International. Vol. 4. 2001, 1853-1855 vol.4. DOI: 10.1109/IGARSS. 2001.977094

[59] Fernanda Lima Kastensmidt. Radiation Effects and Mitigation Techniques for FPGAs. 2012. URL: http: //www. ieav.cta.br/werice2012/Apresenta\%E7\%F5es/ 31/310ut2012\%20-\%20Fernanda\%20Kastensmidt.pdf (visited on 04/01/2014).

[60] A. Kiely and M. Klimesh. "The icer progressive wavelet image compressor". In: IPN Progress Report. 2003, pp. 1-46.

[61] A. Kiely, M. Klimesh, H. Xie, and N. Aranki. "ICER-3D: A Progressive WaveletBased Compressor for Hyperspectral Images". In: (2006).

[62] Bumho Kim, Jeong-Woo Lee, and Ki-Song Yoon. "A parallel implementation of JPEG2000 encoder on multi-GPU system". In: 16th International Conference on Advanced Communication Technology. Global IT Research Institute (GIRI), Feb. 2014, pp. 610-613. ISBN: 978-89-968650-3-2. DOI: 10.1109/ICACT. 2014.6779033. URL: http://ieeexplore.ieee.org/lpdocs/epic03/wrapper.htm?arnumber= 6779033.

[63] K. Kohm. "Modulation Transfer Function Measurement Method and Results for The Orbview-3 High Resolution Imaging Satellite". In: ISPRS Archives - Volume XXXV Part B1, 2004 (2004).

[64] David Krutz. "Ein Betriebssystem für konfigurierbare Hardware". PhD thesis. Humboldt-Universität zu Berlin, Mathematisch-Naturwissenschaftliche Fakultät II: Humboldt-Universität zu Berlin, Nov. 2006.

[65] Krzysztof Kurowski. "Graphics processing unit implementation of JPEG2000 for hyperspectral image compression". In: Journal of Applied Remote Sensing 6.1 (June 2012), p. 061507. ISSN: 1931-3195. DOI: 10.1117/1 . JRS .6.061507. URL: http : / / remotesensing . spiedigitallibrary . org / article . aspx ? articleid $=1352324$.

[66] Catherine Lambert-Nebout, Christophe Latry, Gilles Moury, Christophe Parisot, Marc Antonini, and Michel Barlaud. "On-board Optical Image Compression for Future High Resolution Remote Sensing Systems". In: SPIE Proceedings 4115 (2000), pp. 332-346. DOI: 10.1117/12.411557. 
[67] Roto Le, Iris R. Bahar, and Joseph L. Mundy. "A novel parallel Tier-1 coder for JPEG2000 using GPUs". English. In: 2011 IEEE 9th Symposium on Application Specific Processors (SASP). IEEE, June 2011, pp. 129-136. ISBN: 978-1-4577-12128. DOI: 10.1109/SASP. 2011.5941091. URL: http://www . computer .org/csdl/ proceedings/sasp/2011/1212/00/05941091-abs.html.

[68] Jungwoo Lee. "Optimized quadtree for Karhunen-Loeve transform in multispectral image coding". In: Image Processing, IEEE Transactions on 8.4 (Apr. 1999), pp. 453-461. ISSN: 1057-7149. DOI: 10.1109/83.753734.

[69] Katholieke Universiteit Leuven. Affine Covariant Features. http://www.robots. ox.ac.uk/ vgg/research/affine. 2007. (Visited on 04/01/2014).

[70] Li Li, Gang Zhou, Björn Fiethe, Harald Michalik, and Björn Osterloh. "Efficient implementation of the CCSDS 122.0-B-1 compression standard on a space-qualified field programmable gate array". In: Journal of Applied Remote Sensing 7.1 (2013), pp. 074595-074595. DOI: 10.1117/1.JRS.7.074595. URL: http://dx.doi.org/ 10.1117/1.JRS.7.074595.

[71] Albert Lin. "Hardware Implementation of a Real-Time Image Data Compression for Satellite Remote Sensing". In: Remote Sensing - Advanced Techniques and Platforms (2012), p. 415. DOI: 10.5772/36006.

[72] Albert Lin, Chieh-Fu Chang, Maw-Ching Lin, and Li-Jung Jan. "Field-programmable gate array implementation of Consultative Committee for Space Data Systems image data compression". In: Journal of Applied Remote Sensing 6.1 (2012), pp. 063551/1-063551/13. DOI: 10.1117/1. JRS.6.063551. URL: http://dx.doi . org/10.1117/1. JRS.6.063551.

[73] David G. Lowe. "Distinctive Image Features from Scale-Invariant Keypoints". In: International Journal of Computer Vision 60.2 (Nov. 2004), pp. 91-110. ISSN: 0920-5691. DOI: 10 . 1023 / B : VISI . 0000029664 .99615 . 94. URL: http: //dx.doi.org/10.1023/B:VISI.0000029664.99615.94.

[74] P. Luigi Dragotti, G. Poggi, and A.R.P. Ragozini. "Compression of multispectral images by three-dimensional SPIHT algorithm". In: Geoscience and Remote Sensing, IEEE Transactions on 38.1 (Jan. 2000), pp. 416-428. ISSN: 0196-2892. DOI: $10.1109 / 36.823937$.

[75] Kristian Manthey. "About the impact of CCSDS image data compression on Image Quality". In: 33. Wissenschaftlich-Technische Jahrestagung der DGPF (2013).

[76] Kristian Manthey, David Krutz, and Ben Juurlink. "A new real-time system for image compression on-board satellites". In: ESA/CNES On-Board Payload Data Compression (OBPDC), Venice, Italy (2014).

[77] Kristian Manthey, David Krutz, and Ben Juurlink. "Reconfigurable architecture for real-time image compression on-board satellites". In: Journal of Applied Remote Sensing 9.1 (2015), p. 097497. DOI: $10.1117 / 1$. JRS .9 .097497. URL: http: //dx.doi.org/10.1117/1. JRS.9.097497. 
[78] J Matela. "GPU-Based DWT Acceleration for JPEG2000". In: MEMICS 2009 Proceedings. Brno, 2009, pp. 136-143. ISBN: 978-80-87342-04-6.

[79] Beate Meffert and Olaf Hochmuth. Werkzeuge der Signalverarbeitung. München: Verlag Pearson-Studium, 2004. ISBN: 3827370655.

[80] Mentor Graphics. ModelSim ASIC and FPGA Design - Functional Verification. 2016. URL: https : / /www . mentor . com/products/fv/modelsim/ (visited on 07/01/2016).

[81] Microsemi Corporation. Technology Roadmap for Digital Space Flight Products. 2013. URL: http://www.microsemi.com/document-portal/doc_view/132907technology-roadmap-for-digital-space-flight-products-europe-2013 (visited on 04/01/2014).

[82] Pedram Mohammadi, Abbas Ebrahimi-Moghadam, and Shahram Shirani. "Subjective and Objective Quality Assessment of Image: A Survey". In: CoRR abs/1406.7799 (2014). URL: http://arxiv.org/abs/1406.7799.

[83] Gilles A. Moury and Christophe Latry. In-orbit commissioning of SPOT5 image compression function. 2003. DOI: 10.1117/12.503919. URL: http://dx . doi . org/10.1117/12.503919.

[84] Nor Noor and Tanya Vladimirova. "Integer KLT Design Space Exploration for Hyperspectral Satellite Image Compression". In: Convergence and Hybrid Information Technology. Ed. by Geuk Lee, Daniel Howard, and Dominik Slezak. Vol. 6935. Lecture Notes in Computer Science. Springer Berlin / Heidelberg, 2011, pp. 661-668. ISBN: 978-3-642-24081-2. URL: http://dx.doi.org/10.1007/9783-642-24082-9_80.

[85] W.A. Pearlman, A. Islam, N. Nagaraj, and A. Said. "Efficient, low-complexity image coding with a set-partitioning embedded block coder". In: Circuits and Systems for Video Technology, IEEE Transactions on 14.11 (Nov. 2004), pp. 1219 1235. ISSN: 1051-8215. DOI: 10.1109/TCSVT. 2004.835150.

[86] Nikolay Ponomarenko, Vladimir Lukin, Alexander Zelensky, Karen Egiazarian, Jaakko Astola, Marco Carli, and Federica Battisti. "TID2008 - A Database for Evaluation of FullReference Visual Quality Assessment Metrics". In: Advances of Modern Radioelectronics Vol. 10 (2009), pp. 30-45.

[87] Shen-En Qian. "Hyperspectral data compression using a fast vector quantization algorithm". In: Geoscience and Remote Sensing, IEEE Transactions on 42.8 (Aug. 2004), pp. 1791-1798. ISSN: 0196-2892. DOI: 10.1109/TGRS. 2004.830126.

[88] Shen-En Qian, M. Bergeron, I. Cunningham, L. Gagnon, and A. Hollinger. "Near lossless data compression onboard a hyperspectral satellite". In: Aerospace and Electronic Systems, IEEE Transactions on 42.3 (July 2006), pp. 851-866. ISSN: 0018-9251. DOI: 10.1109/TAES. 2006.248183. 
[89] Sunil Chokkanathapuram Ramanarayanan, Kristian Manthey, and Ben Juurlink. "High performance CCSDS image data compression using GPGPUs for space applications". In: 26. GI/ITG Workshop Parallel-Algorithmen,-Rechnerstrukturen und-Systemsoftware (PARS), Universitaet Potsdam, Potsdam, Germany (2015).

[90] Jörg Ritter and Paul Molitor. "A pipelined architecture for partitioned DWT based lossy image compression using FPGAs". In: Proceedings of the 2001 ACM/SIGDA ninth international symposium on Field programmable gate arrays. FPGA '01. Monterey, California, United States: ACM, 2001, pp. 201-206. ISBN: 1-58113341-3. DOI: http : / / doi . acm . org/10 . 1145/360276 . 360350. URL: http : //doi.acm.org/10.1145/360276.360350.

[91] D. Rueß, A. Luber, K. Manthey, and R. Reulke. "Accuracy evaluation of stereo camera systems with generic camera models". In: ISPRS - International Archives of the Photogrammetry, Remote Sensing and Spatial Information Sciences XXXIXB5 (2012), pp. 375-380. DOI: 10 .5194/isprsarchives-XXXIX-B5-375-2012. URL: http : / www . int-arch-photogramm-remote-sens-spatial-inf-sci . net/XXXIX-B5/375/2012/.

[92] M.J. Ryan and J.F. Arnold. "The lossless compression of AVIRIS images by vector quantization". In: Geoscience and Remote Sensing, IEEE Transactions on 35.3 (May 1997), pp. 546-550. ISSN: 0196-2892. DOI: 10.1109/36.581964.

[93] J.A. Saghri, A.G. Tescher, and J.T. Reagan. "Practical transform coding of multispectral imagery". In: Signal Processing Magazine, IEEE 12.1 (Jan. 1995), pp. 32-43. ISSN: 1053-5888. DOI: 10.1109/79.363506.

[94] Amir Said and William A. Pearlman. "A new, fast, and efficient image codec based on set partitioning in hierarchical trees". In: IEEE Transactions on Circuits and Systems for Video Technology 6.3 (June 1996), pp. 243-250.

[95] M. Santhi and R. S. D. Wahida Banu. "Modified SPIHT Algorithm for Coding Color Images using Inter-color Correlation". In: (2010).

[96] Satellite Imaging Corporation. WorldView-2 Satellite Sensor. URL: http://www . satimagingcorp . com/ satellite-sensors/worldview-2.html (visited on 04/01/2014).

[97] Satellite Imaging Corporation. WorldView-3 Satellite Sensor. URL: http://www . satimagingcorp . com/satellite-sensors/worldview-3.html (visited on 04/01/2014).

[98] D. Scharstein and R. Szeliski. "High-accuracy stereo depth maps using structured light". In: 2003 IEEE Computer Society Conference on Computer Vision and Pattern Recognition, 2003. Proceedings. Vol. 1. June 2003, pp. 195-202. DOI: 10.1109/CVPR. 2003.1211354.

[99] Daniel Scharstein and Richard Szeliski. "A Taxonomy and Evaluation of Dense Two-Frame Stereo Correspondence Algorithms". In: Int. J. Comput. Vision 47.1-3 (Apr. 2002), pp. 7-42. ISSN: 0920-5691. DOI: 10.1023/A : 1014573219977. URL: http://dx.doi.org/10.1023/A:1014573219977. 
[100] e2v semiconductors. P4080 QorIQ Integrated Processor Hardware Specifications, Datasheet Summary. 2011. URL: http : / / www . msc-ge . com / de /6798-www / version / default/part / AttachmentData / data / P4080.pdf ? language =en (visited on $04 / 01 / 2014$ ).

[101] Claude Shannon. "A Mathematical Theory of Communication". In: Bell System Technical Journal 27 (1948), pp. 379-423, 623-656. URL: http:// cm . belllabs.com/cm/ms/what/shannonday/shannon1948.pdf.

[102] J.M. Shapiro. "Embedded image coding using zerotrees of wavelet coefficients". In: Signal Processing, IEEE Transactions on 41.12 (Dec. 1993), pp. 3445-3462. ISSN: 1053-587X. DOI: 10.1109/78.258085.

[103] Changhe Song, Yunsong Li, and Bormin Huang. "A GPU-Accelerated Wavelet Decompression System With SPIHT and Reed-Solomon Decoding for Satellite Images". In: IEEE Journal of Selected Topics in Applied Earth Observations and Remote Sensing 4.3 (Sept. 2011), pp. 683-690. ISSN: 1939-1404. DOI: 10.1109/ JSTARS . 2011 . 2159962. URL: http : / / www . researchgate . net/publication/ $224250312 \% 5 C_{-}$A $\% 5 C$ C GPU-Accelerated $\% 5$ C_Wavelet $\% 5 C_{-}$Decompression $\% 5 C$ _ System $\% 5$ C_With $\% 5 C$ _SPIHT $\% 5 C$ _and $\% 5 C$ Reed-Solomon $\% 5 C$ Decoding $\% 5 C$ for $\%$ 5 C_Satellite $\% 5$ C_Images.

[104] space.skyrocket.de. AISSat 1, 2, 3. URL: http://space.skyrocket.de/doc_ sdat/aissat-1.htm (visited on 04/01/2014).

[105] N. Sriraam and R. Shyamsunder. "3-D medical image compression using 3-D wavelet coders". In: Digit. Signal Process. 21 (1 Jan. 2011), pp. 100-109. ISSN: 1051-2004. DOI: http://dx.doi .org/10.1016/j.dsp.2010.06.002. URL: http://dx.doi.org/10.1016/j.dsp.2010.06.002.

[106] M. Stettler, M. Caffre, P. Graham, and J. Krone. "Radiation effects and mitigation strategies for modern FPGA". In: 10th Workshop on Electronics for LHC Experiments and Future Experiments. Sept. 2004.

[107] Tilo Strutz. Bilddatenkompression, Grundlagen, Codierung, Wavelets, JPEG, MPEG, H.264. Wiesbaden: Vieweg+Teubner, June 2005. ISBN: 3528239220.

[108] R. Sudhakar, R. Karthiga, and S. Jayaraman. "Image Compression Using Coding of Wavelet Coefficients: A Survey". In: ICGST-GVIP Journal 5.6 (2005), pp. 2538.

[109] Wim Sweldens. "The lifting scheme: A new philosophy in biorthogonal wavelet constructions". In: Wavelet Applications in Signal and Image Processing III. 1995, pp. $68-79$.

[110] The MathWorks, Inc. MathWorks MATLAB - The Language of Technical Computing. 2016. URL: https://www. mathworks. com/products/matlab/index.html (visited on $07 / 01 / 2016$ ). 
[111] C. Thiebaut, D. Lebedeff, C. Latry, and Y. Bobichon. "On-board compression algorithm for satellite multispectral images". In: Data Compression Conference, 2006. DCC 2006. Proceedings. Mar. 2006. DOI: 10.1109/DCC.2006.61.

[112] Carole Thiebaut, Xavier Delaunay, Christophe Latry, and Gilles Moury. "CNES studies for on-board compression of high-resolution satellite images". In: Proc. SPIE 7084 (2008), 70840F-70840F/8. DOI: $10.1117 / 12.798121$. URL: http: //dx.doi.org/10.1117/12.798121.

[113] Jun Tian and Raymond O Wells Jr. "Embedded image coding using wavelet difference reduction". In: Wavelet image and video compression 450 (1998), pp. 289301.

[114] Roland Trautner. "ESA's Roadmap for Next Generation Payload Data Processors". In: DASIA. 2011.

[115] Roland Trautner and Raffaele Vitulli. "Ongoing Developments of Future Payload Data Processing Platforms at ESA". In: OBPDC. 2010.

[116] C. Valens. EZW encoding. 2004. URL: http://polyvalens.pagesperso-orange. $\mathrm{fr} / \mathrm{clemens} /$ ezw/ezw.html.

[117] James S. Walker. "Lossy image codec based on adaptively scanned wavelet difference reduction". In: Optical Engineering 39.7 (2000), pp. 1891-1897. DOI: 10.1117/1.602573. URL: http://dx.doi.org/10.1117/1.602573.

[118] Hongqiang Wang. CCSDS Image Data Compression Implementation. 2008. URL: http://hyperspectral .unl.edu (visited on 04/01/2014).

[119] Hongqiang Wang, S.D. Babacan, and K. Sayood. "Lossless Hyperspectral-Image Compression Using Context-Based Conditional Average". In: Geoscience and Remote Sensing, IEEE Transactions on 45.12 (Dec. 2007), pp. 4187-4193. ISSN: 0196-2892. DOI: 10.1109/TGRS . 2007.906085.

[120] Zhou Wang, A.C. Bovik, H.R. Sheikh, and E.P. Simoncelli. "Image quality assessment: from error visibility to structural similarity". In: Image Processing, IEEE Transactions on 13.4 (Apr. 2004), pp. 600-612. ISSN: 1057-7149. DOI: 10.1109/TIP. 2003.819861.

[121] Wikipedia. Angular resolution. 2006. URL: https://en.wikipedia.org/wiki/ Lens_\%28optics\%29\#Imaging_properties/ (visited on 04/01/2014).

[122] Wikipedia. Lens3. 2006. URL: https : / / en . wikipedia . org/wiki / Lens _ \%28optics\%29\#Imaging_properties/ (visited on 04/01/2014).

[123] Wikipedia. Huffman coding. URL: https://en.wikipedia.org/wiki/Huffman_ coding (visited on 04/01/2014).

[124] Wikipedia. Reaction wheel. URL: https://en.wikipedia.org/wiki/Reaction_ wheel/ (visited on 04/01/2014). 
[125] J.A. Williams, A.S. Dawood, and S.J. Visser. "FPGA-based cloud detection for real-time onboard remote sensing". In: Field-Programmable Technology, 2002. (FPT). Proceedings. 2002 IEEE International Conference on. Dec. 2002, pp. 110 116. DOI: $10.1109 /$ FPT. 2002.1188671.

[126] P. Winterrowd, C. Orbe, J. Venbrux, S. Whitaker, E. Cameron, R. Nelson, G. Maki, D. Fisher, and Pen-Shu Yeh. "A 320 Mbps flexible image data compressor for space applications". In: Aerospace Conference, 2010 IEEE. Mar. 2010, pp. 1-6. DOI: $10.1109 /$ AERO .2010 .5446664 .

[127] Xilinx, Inc. Radiation Effects and Mitigation Overview. 2004. URL: http://www. xilinx.com/esp/mil_aero/collateral/presentations/radiation_effects. pdf (visited on 04/01/2014).

[128] Xilinx, Inc. Radiation-Hardened, Space-Grade Virtex-5QV Device Overview. 2011. URL: http://www.xilinx.com/support/documentation/data_sheets/ds192_ V5QV_Device_Overview.pdf (visited on 04/01/2014).

[129] Xilinx, Inc. Calculating Toggle Rates. 2013. URL: http://www.xilinx.com/ise/ power_tools/wpt_help/app_docs/calculating_toggle_rates.htm (visited on $04 / 01 / 2016)$.

[130] Xilinx, Inc. Xilinx Power Estimator (XPE). 2013. URL: http://www .xilinx . com/products/design_tools/logic_design/xpe.htm (visited on 04/01/2014).

[131] Xilinx, Inc. Virtex-5 FPGA Data Sheet: DC and Switching Characteristics. 2016. URL: http://www . xilinx.com/support/documentation/data_sheets/ds160. pdf (visited on 04/01/2016).

[132] Xilinx, Inc. Xilinx PCI Express (PCIe). 2016. URL: http://www.xilinx.com/ products/technology/pci-express.html (visited on 07/01/2016).

[133] Pen-Shu Yeh, P. Armbruster, A. Kiely, B. Masschelein, G. Moury, C. Schaefer, and C. Thiebaut. "The new CCSDS image compression recommendation". In: Aerospace Conference, 2005 IEEE. Mar. 2005, pp. 4138-4145. DOI: 10.1109/AERO. 2005.1559719.

[134] Guoxia Yu, Tanya Vladimirova, and Martin N. Sweeting. "Image compression systems on board satellites". In: Acta Astronautica64 64 (2009), pp. 988-1005. DOI: http://dx.doi.org/10.1016/j.actaastro.2008.12.006. 


\section{A Additional Information}

\section{A.1 Hardware Design}

Today's systems are most often heterogeneous systems, i. e. they combine hardware and software components. Their advantage is that they can be efficiently adapted to the application's requirements. As a logical consequence, the design of heterogeneous systems is not limited to just hardware or just software design [64]. This in turn leads to a complex system model which is used to generate the system components.

In principle, there are three major aspects in the development of heterogeneous systems:

- A system model is used as a conceptual model that describes and represents the system. On one hand, it abstracts the underlying hardware, but on the other hand, it must permit an optimal implementation.

- The system partitioning involves the decomposition of the application to the individual components of the heterogeneous system, and their communication with each other.

- System verification is the process of testing the system functions against a requirements document. Each requirement should be testable, and all requirements should be tested.

The Gajski-Kuhn Y-chart is a model that describes the considerations in hardware design, especially for semiconductor devices [40]. The model describes that hardware design is performed in three domains. Each of the domains are divided into levels of abstraction, using concentric rings. The three main aspects of geometry, behavior and structure of the various levels of abstraction are reflected in the diagram. The behavioral domain describes the functionality of the system, which is implemented by the geometry or physical domain. The design process proposes to describe the behavior of the heterogeneous system at the highest level. The behavior model is transformed into a structural description through the processes of high-level synthesis or logical synthesis. With the help of layout synthesis tools it is possible to transfer structural description into the logic level or physical description.

\section{A.2 Pipe Communication Protocol}

The following source code shows the fundamentals of the pipe communication protocol presented in [64]. Data is read from an input pipe, inverted and written to an output 


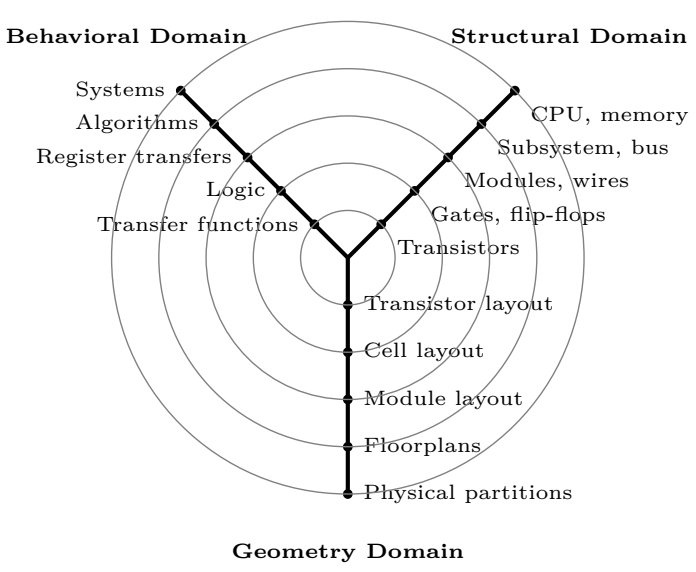

(a) Abstraction levels

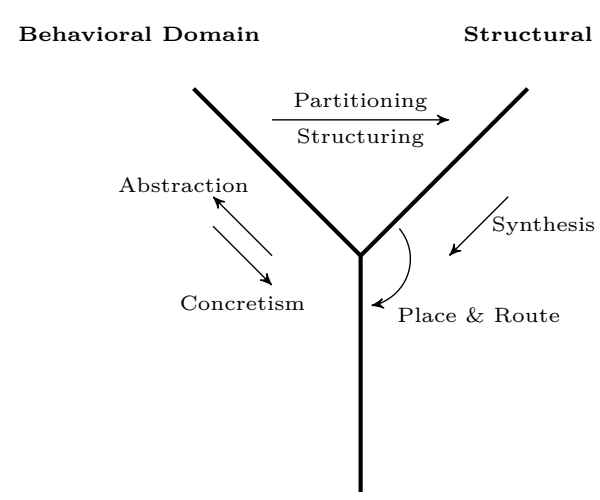

Geometry Domain

(b) Design process

Figure A.1: The Gajski-Kuhn Y-chart model [40].

pipe. The two-process method proposed in [39] is used to enhance readability and to reduce the error rate of the code.

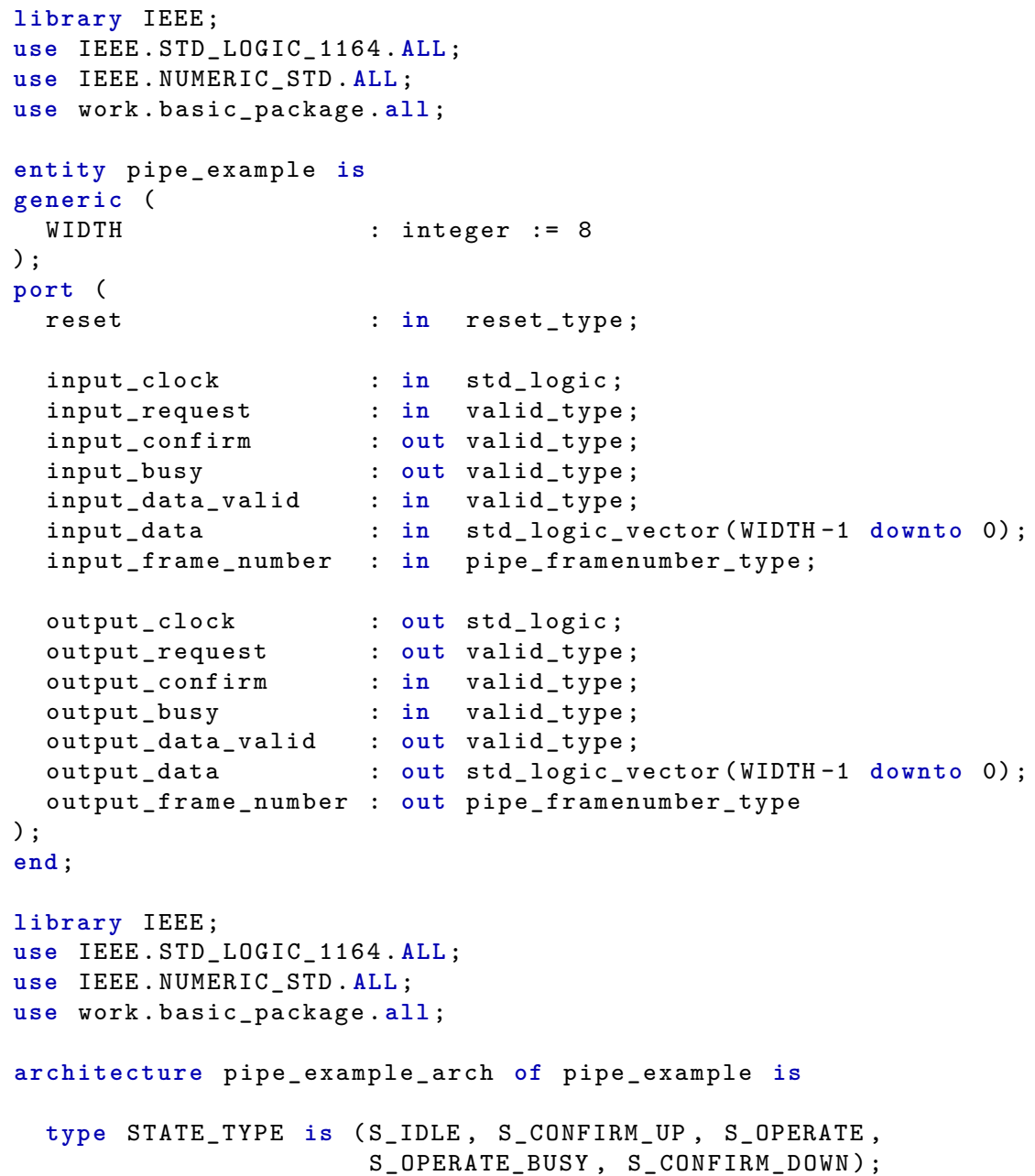




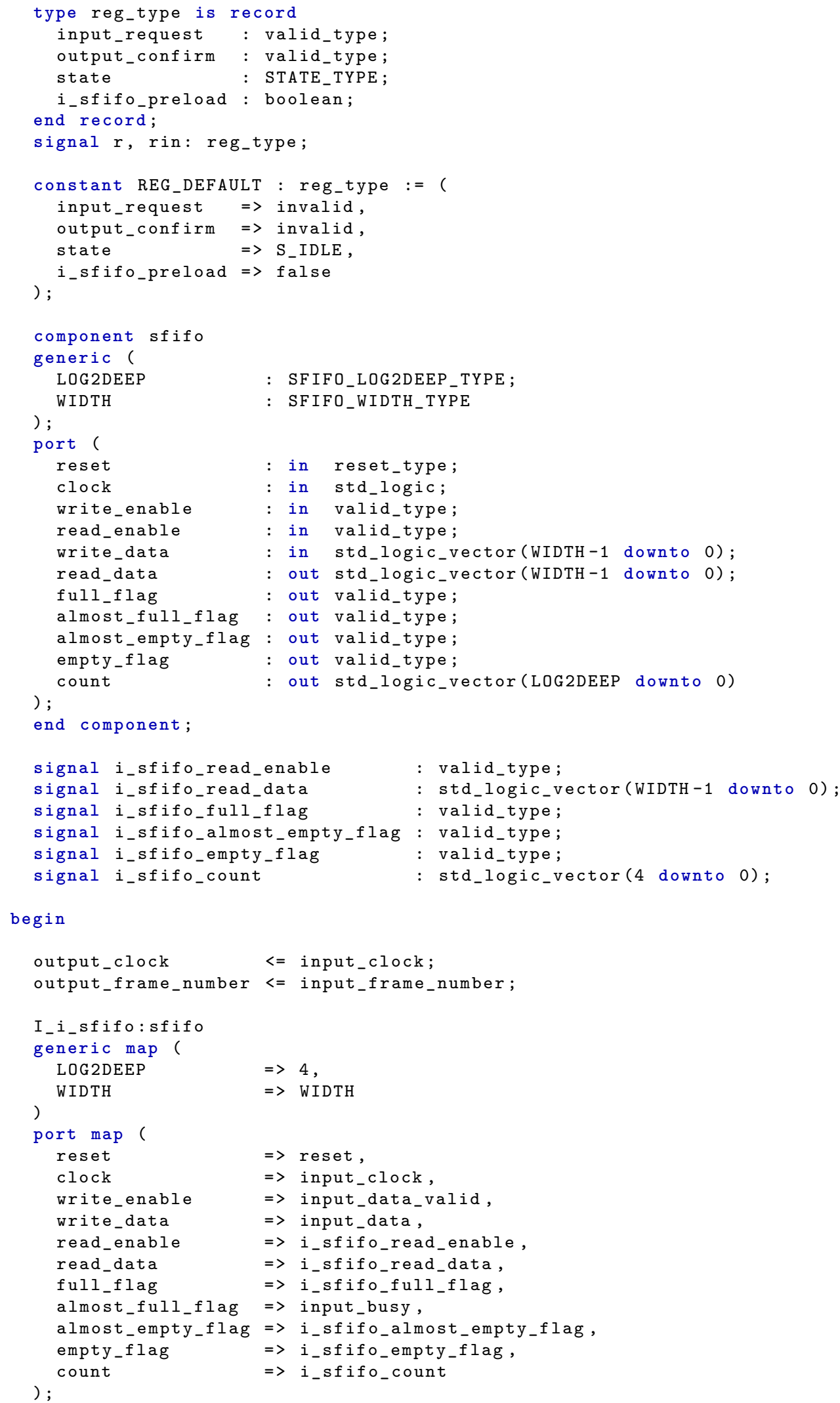




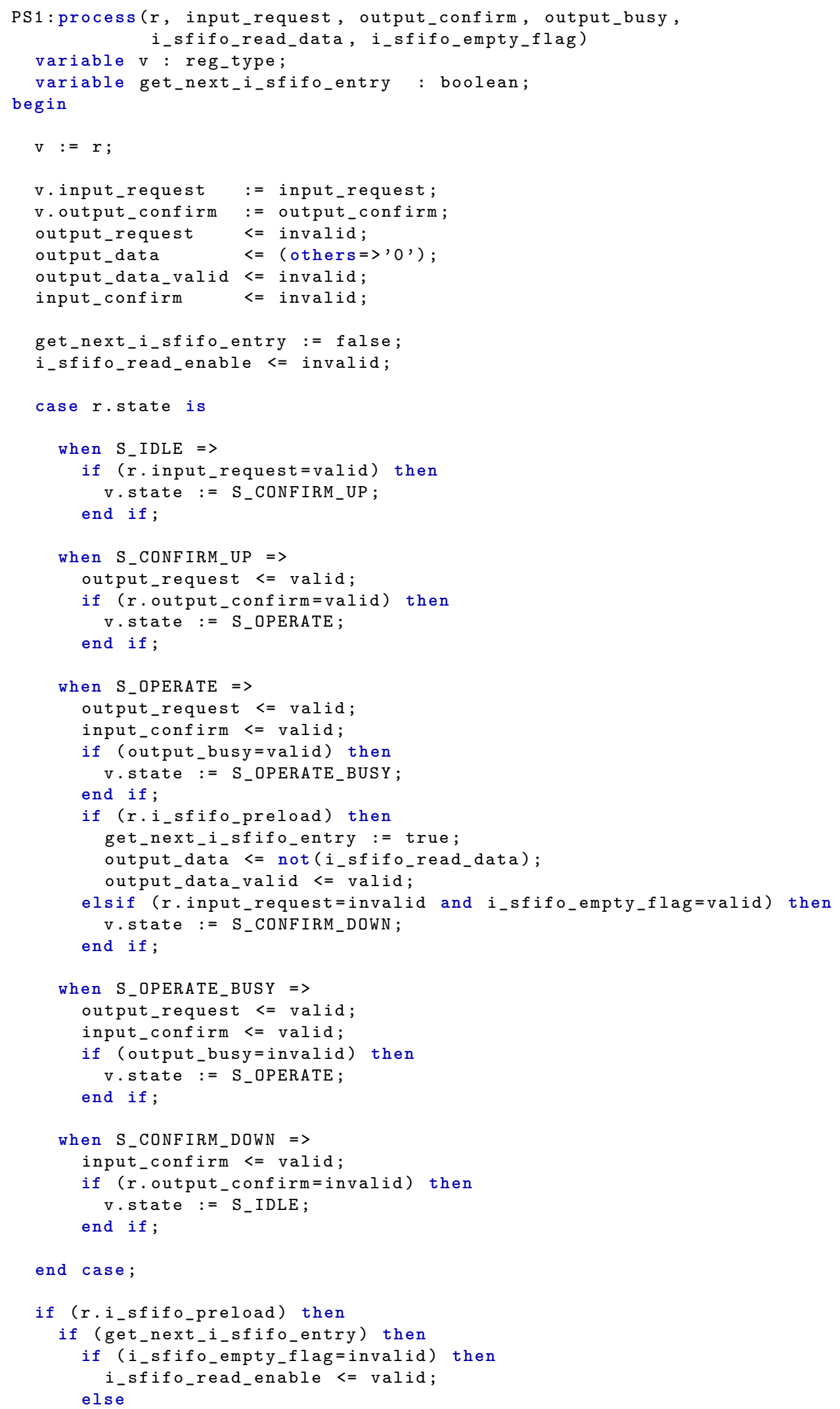




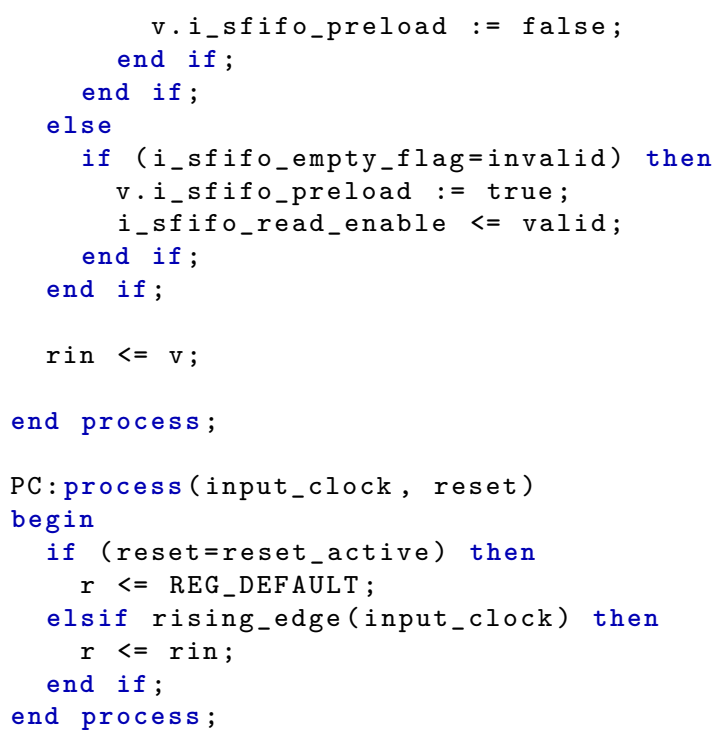




\section{A.3 Row Integer DWT}

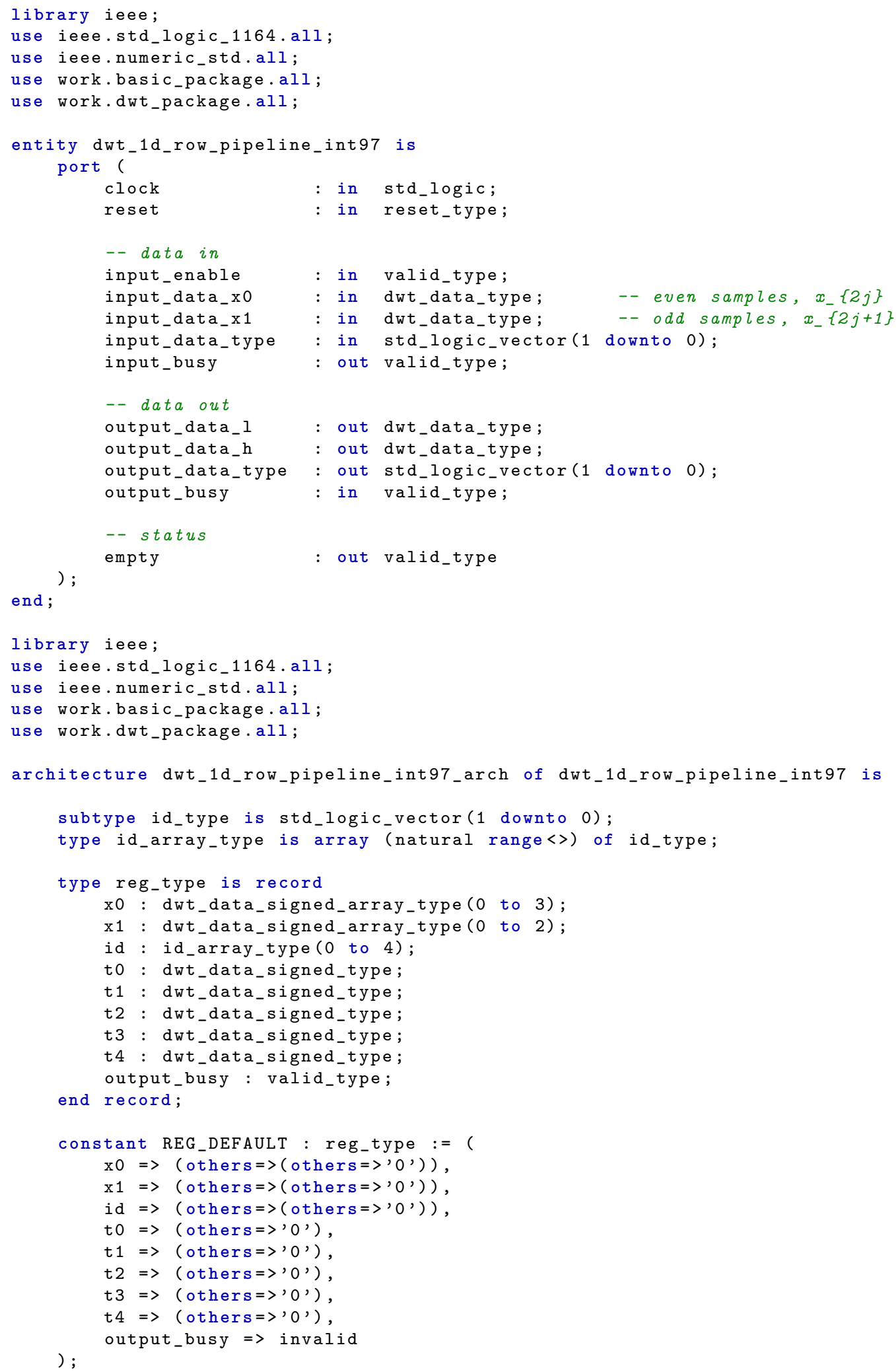


signal r, rin: reg_type;

begin

input_busy $<=$ output_busy;

PS1:process ( $r$, input_enable, input_data_x0, input_data_x1, input_data_type, output_busy)

variable v : reg_type;

variable tmp : dwt_data_signed_type;

begin

$\mathrm{v}:=\mathrm{r}$;

output_data_l $<=\left(\right.$ others $\left.=>^{\prime} 0^{\prime}\right)$;

output_data_h $<=\left(\right.$ others $\left.=>^{\prime} 0^{\prime}\right)$;

output_data_type $<=$ (others= $\left.>^{\prime} 0^{\prime}\right)$;

- generate empty flag

empty<= valid;

for $I$ in 0 to 4 loop

if $(r . i d(I) /=" 00 ")$ then

end if

empty <= invalid;

end loop;

-- pipeline...

v.output_busy := output_busy;

if (r.output_busy=invalid) then

if (input_enable=valid) then

- output data

output_data_l $<$ std_logic_vector(r.t4);

output_data_h $<$ std_logic_vector(r.t3);

output_data_type $<=r$.id (4);

- register input

$\mathrm{v} \cdot \mathrm{x} 0(0):=$ signed (input_data_x0);

$\mathrm{v} \cdot \mathrm{x} 1(0):=$ signed (input_data_x1);

v.id(0) := input_data_type;

for $I$ in 1 to 3 loop

$\mathrm{v} \cdot \mathrm{xO}(\mathrm{I}):=\mathrm{r} \cdot \mathrm{x} 0(\mathrm{I}-1)$;

end loop;

for $I$ in 1 to 2 loop

$\mathrm{v} \cdot \mathrm{x} 1(\mathrm{I}):=\mathrm{r} \cdot \mathrm{x} 1(\mathrm{I}-1)$;

end loop;

for $I$ in 1 to 4 loop

$\mathrm{v} . \mathrm{id}(\mathrm{I}):=\mathrm{r} \cdot \mathrm{id}(\mathrm{I}-1)$;

end loop;

- stage 0

tmp : $=r \cdot x 0(0)+r \cdot x 0(1)$;

$\mathrm{v} \cdot \mathrm{t} 0:=$ shift_left(tmp, 3$)+\mathrm{tmp}$;

)

$\mathrm{v} \cdot \mathrm{t} 1:=r \cdot \mathrm{x} 0(2)+\operatorname{signed}($ input_data_x0);

$--9 *\left(x_{-}\{2 j\}+x_{-}\{2 j+2\}\right.$

- stage 1

tmp : : r.to - r.t 1 ;

)$-x_{-}\{2 j-2\}+x_{-}\{2 j+4\}$

tmp := shift_right_round (tmp, 4); $\quad-I_{-} t m p+1 / 2 \ldots l$

$\mathrm{v} \cdot \mathrm{t} 2:=r \cdot \mathrm{x} 1(2)-\mathrm{tmp}$;

+1\})

$--x_{-}\{2 j-2\}+x_{-}\{2 j+4\}$

-- stage 2 


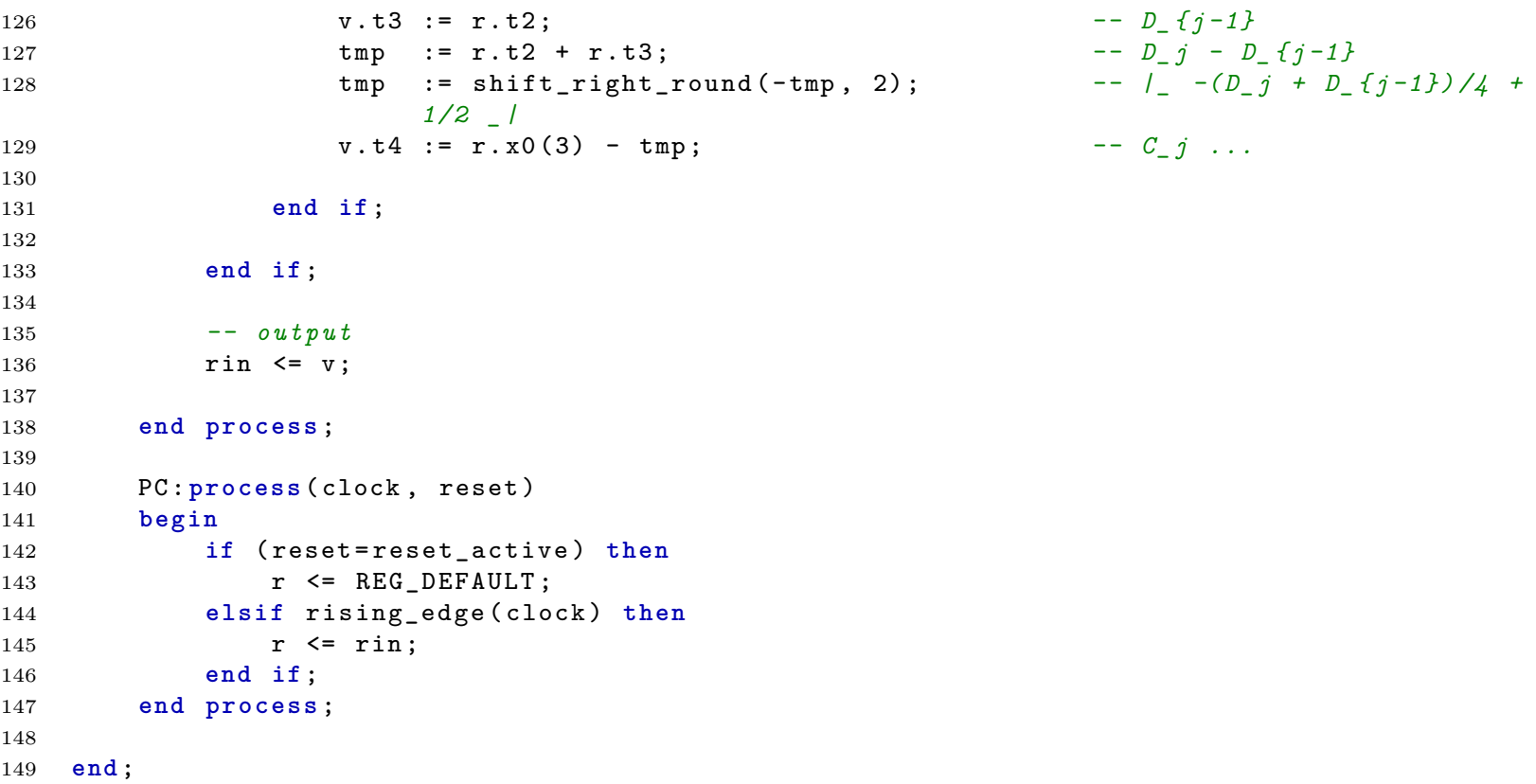




\section{A.4 Row Floating Point DWT}

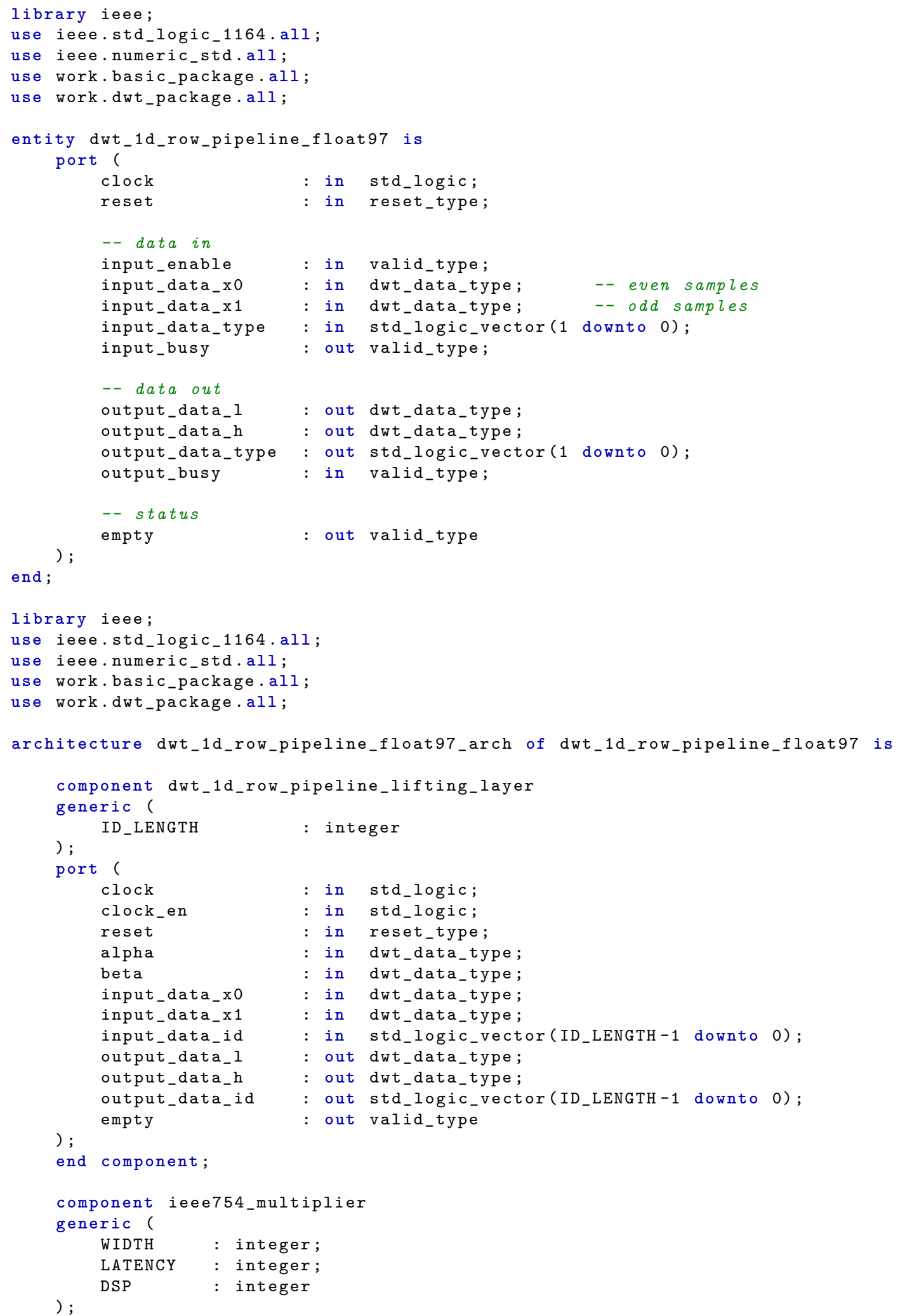




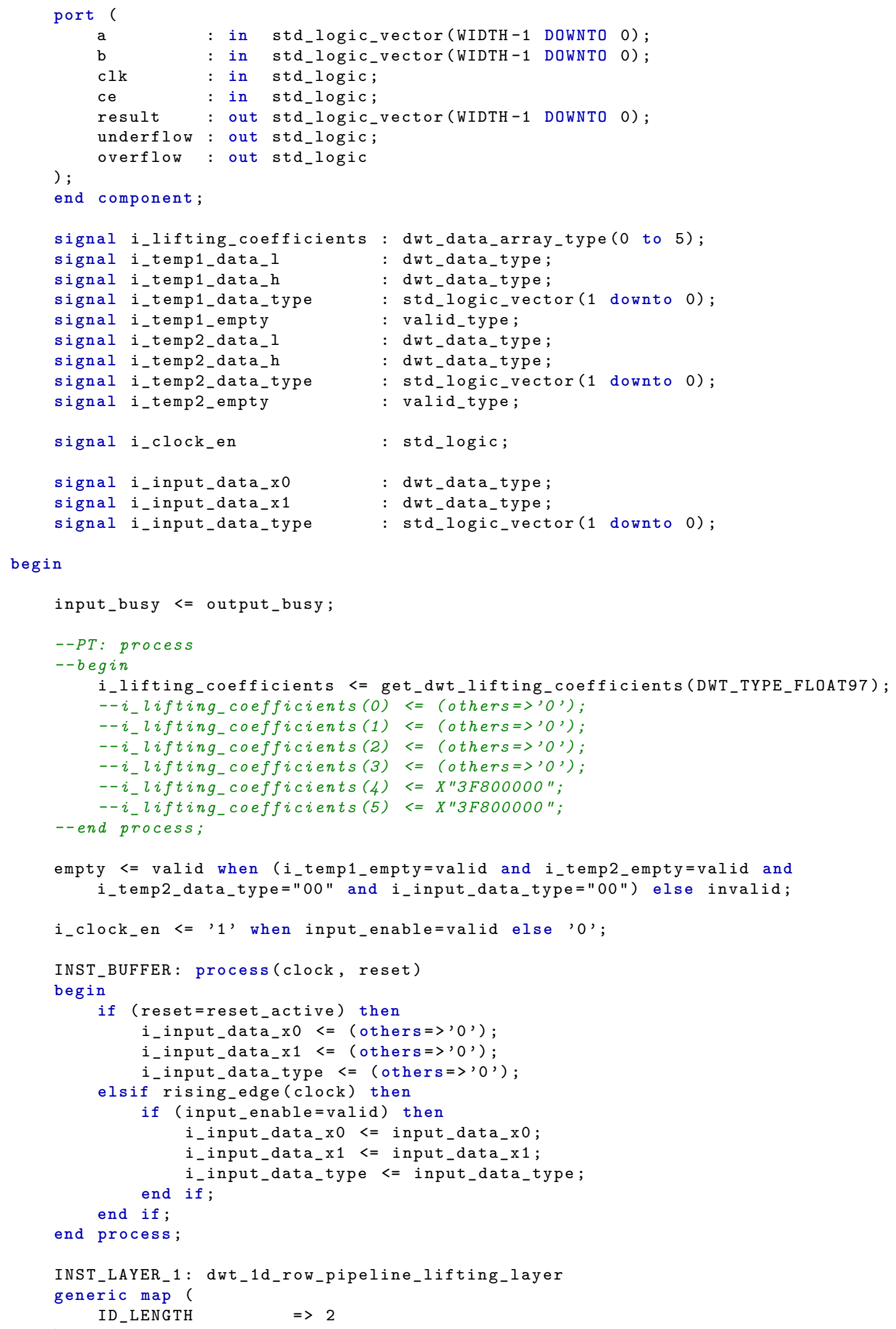




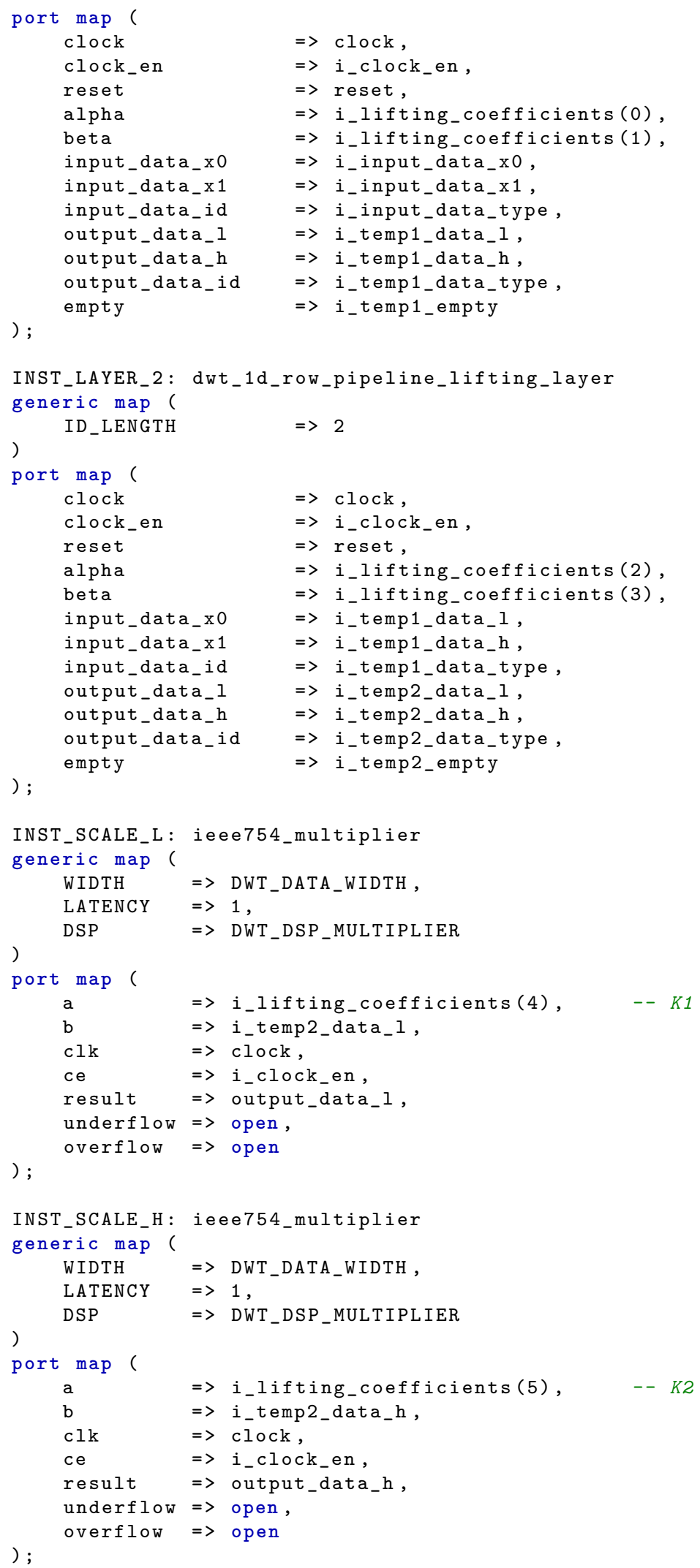




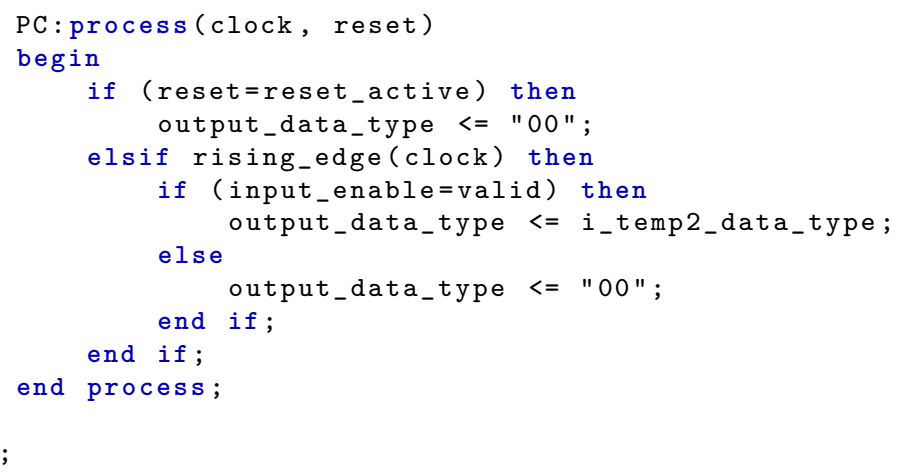




\section{A.5 Row Floating Point DWT (Lifting Layer)}

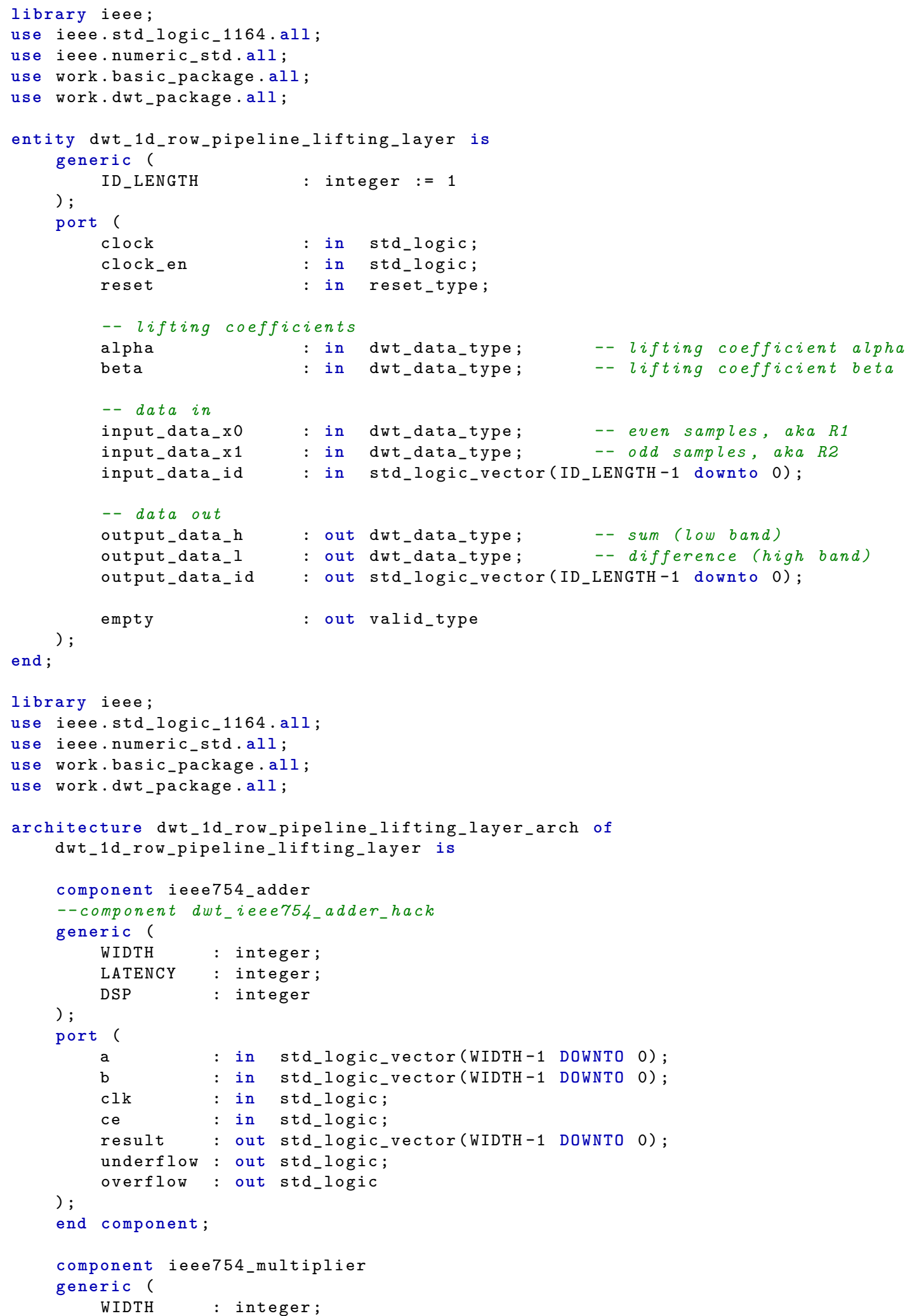




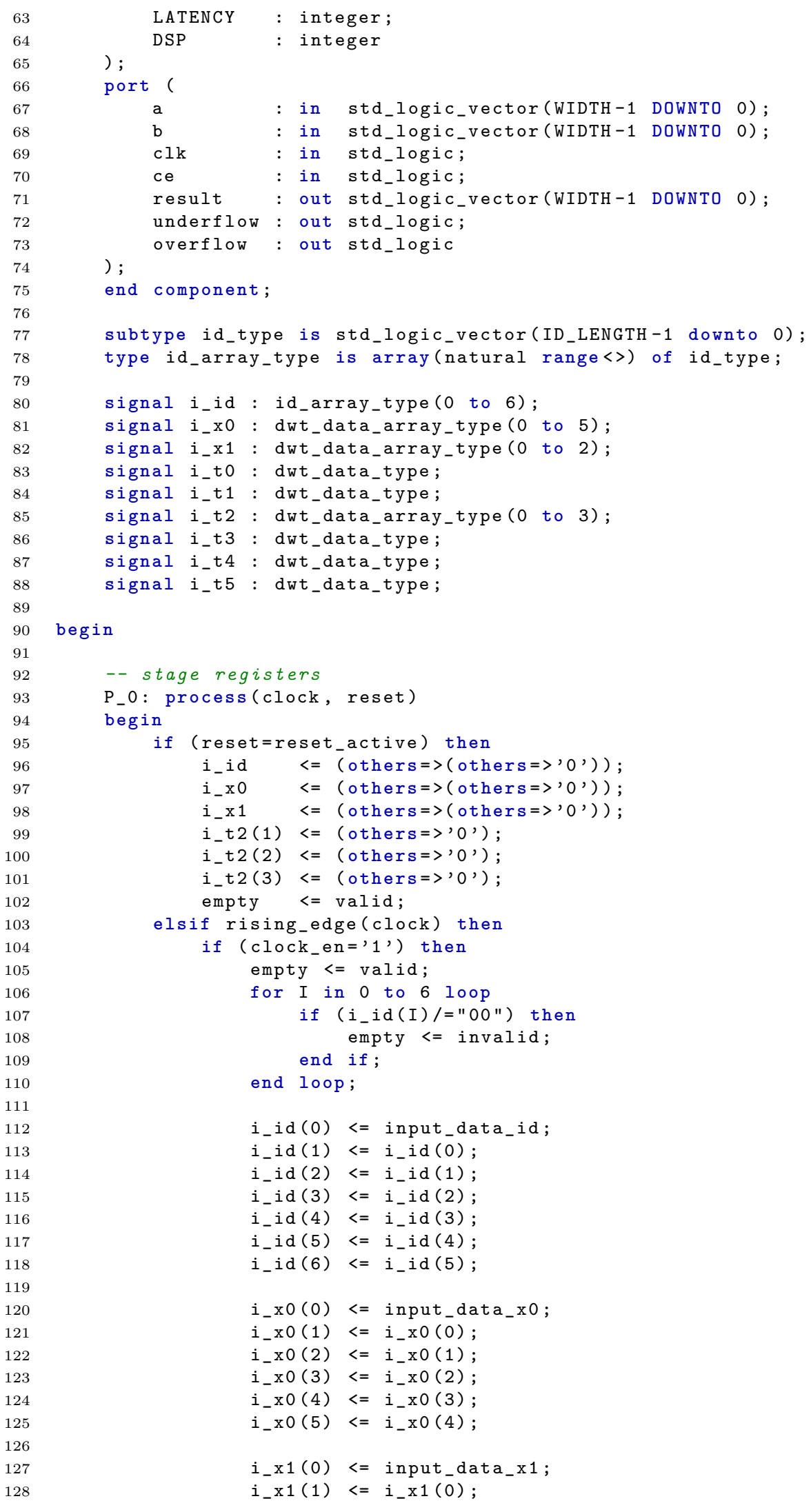




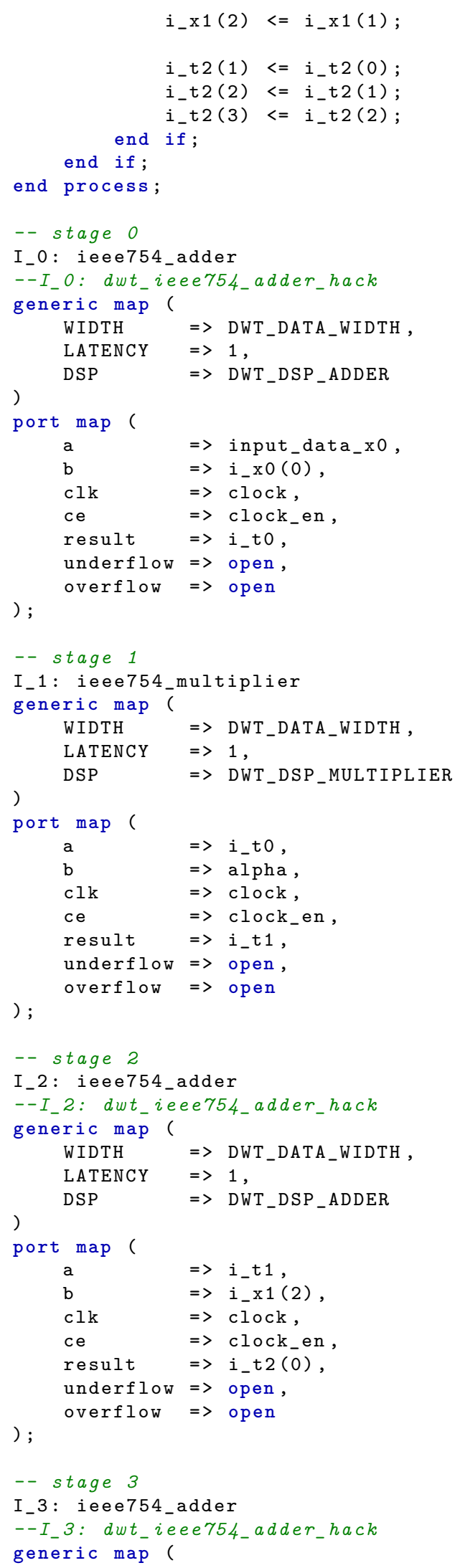




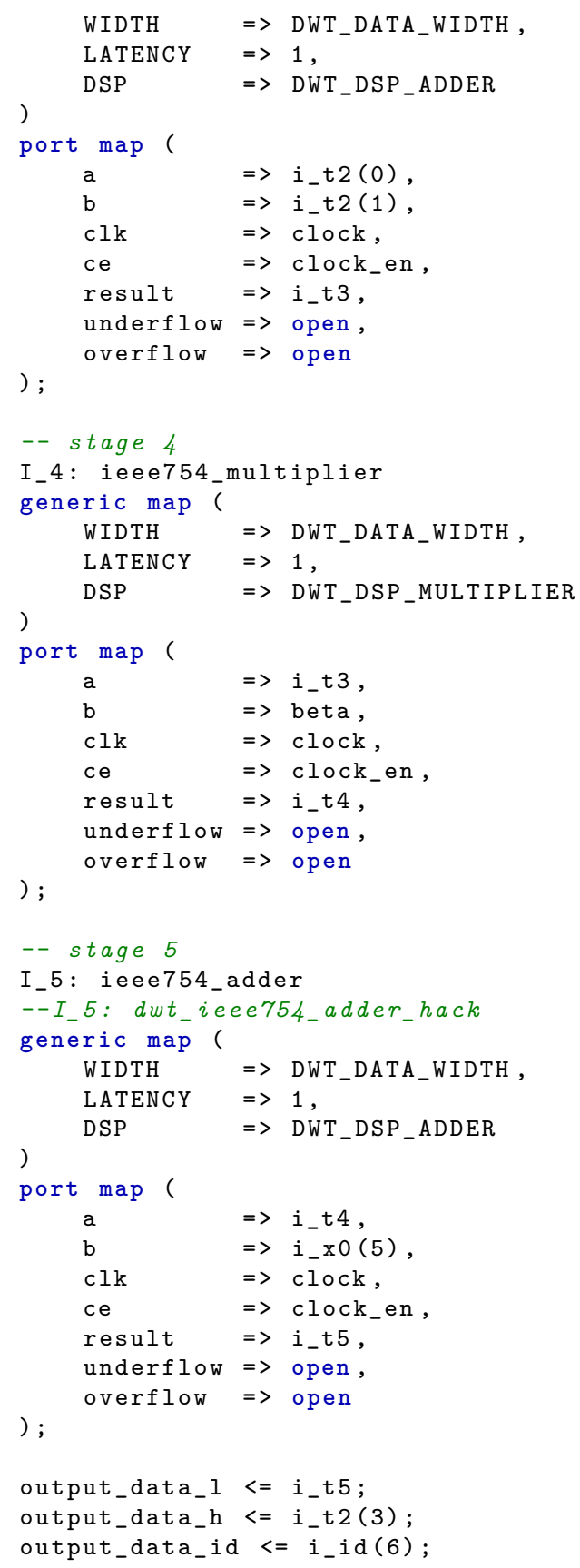




\section{B Test Images}

For evaluation, verification and test, various test images presented in this section are used.

\section{B.1 Synthetic Images}

To investigate in the impact of image compression on image noise, Gaussian noise images $I_{n}(\sigma, \mu)$ with $\sigma^{2}=\{10,20,40,80,160\}$ and $\mu=128$ were generated. Gaussian noise images were calculated with the following Matlab script. A refinement loop is used to adjust the image noise variance after rounding to the nearest integer.

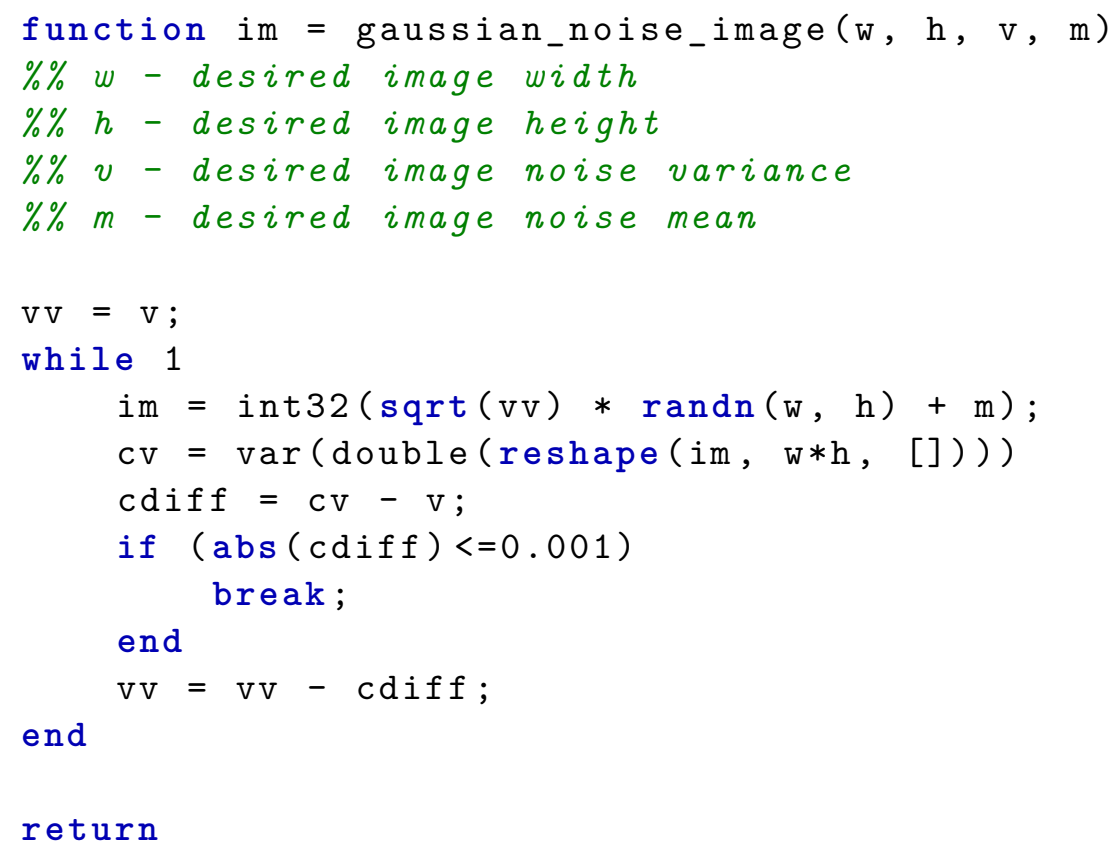

Images with uniform distributed random values were calculated as follows:

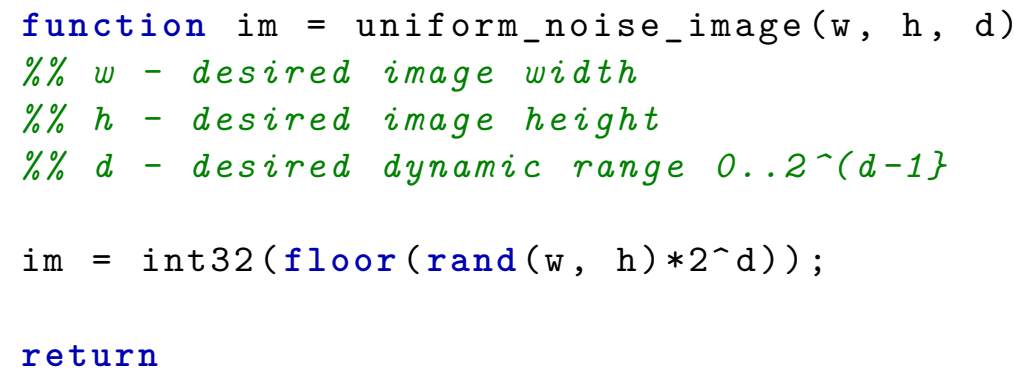


In the case of compression on noisy images, Gaussian noise was added to a reference images. The variance of the noise is $\sigma^{2}=\{50,200\}$ and the mean is zero.

\section{B.2 Leuven Images}

To investigate in the impact of image compression on feature matching, test images bark, graf, trees and wall of the university Leuven [69] were used. The datasets contain several images taken from different perspectives and the corresponding homographies between image pairs. The test images bark, graf, trees, and wall were used.

\section{B.3 Middlebury Images}

For the investigation in the impact of image compression on stereo matching, images from the Middlebury Stereo Vision Page [98, 99] were used. 


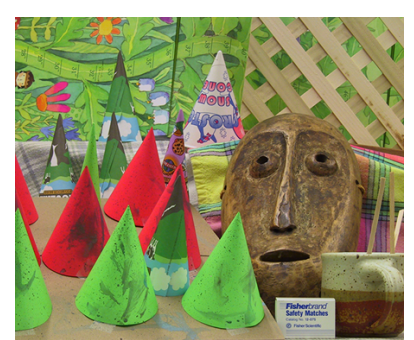

Cones (left)

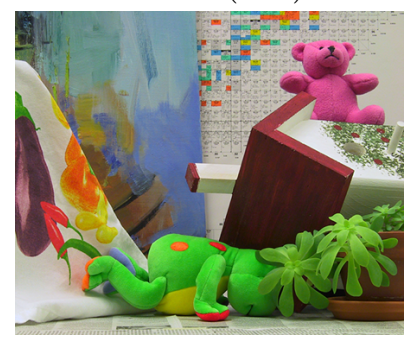

Teddy (left)

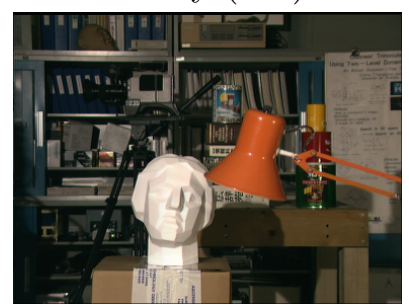

Tsukuba (left)

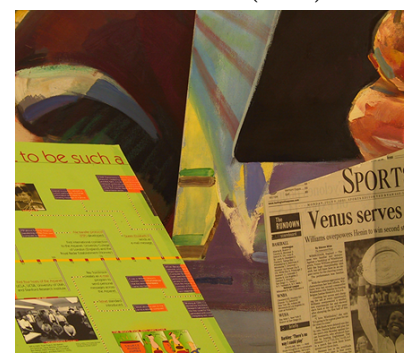

Venus (left)

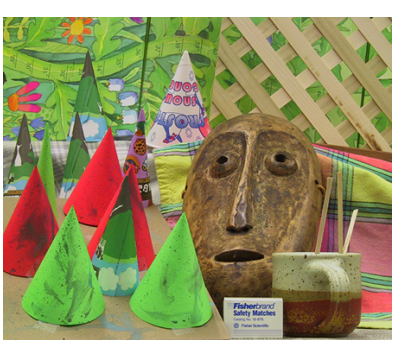

Cones (right)

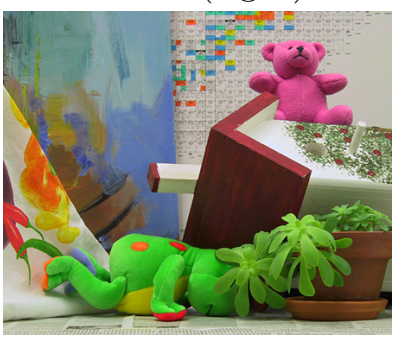

Teddy (right)

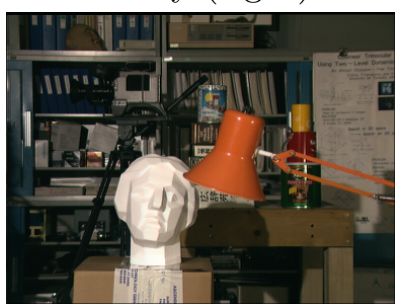

Tsukuba (right)

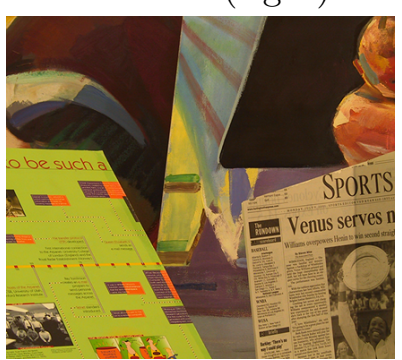

Venus (right)

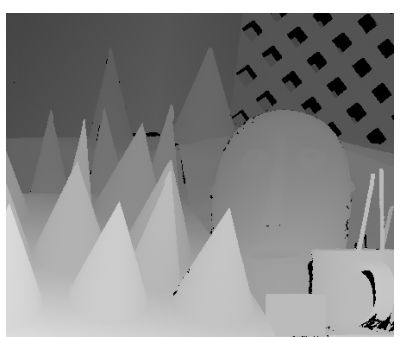

Cones (ground truth)

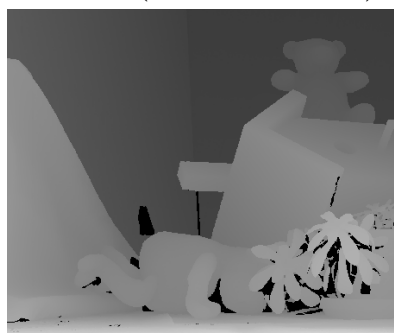

Teddy (ground truth)

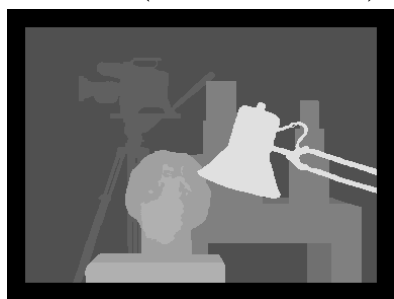

Tsukuba (ground truth)

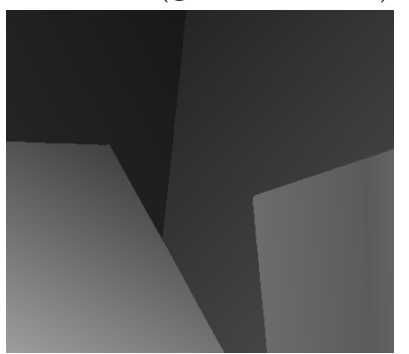

Venus (ground truth)

Figure B.1: Middlebury Stereo Vision test images. 


\section{B.4 CCSDS Reference Image Set}

Table B.1 is taken from [25]. Copyright of the images is acknowledged as follows:

"Copyright xxx. ${ }^{1}$ All rights reserved. This image may be used and distributed without restrictions provided that this copyright statement is retained and that any derivative work acknowledges the origin of the information."

\begin{tabular}{|c|c|c|c|}
\hline Image Name & Source-Copyright & Size $(h \times w)$ & Dynamic range [bpp] \\
\hline coastal_b1 & Landsat - NASA & $1024 \times 1024$ & 8 \\
\hline coastal_b2 & Landsat - NASA & $1024 \times 1024$ & 8 \\
\hline coastal_b3 & Landsat - NASA & $1024 \times 1024$ & 8 \\
\hline coastal_b4 & Landsat - NASA & $1024 \times 1024$ & 8 \\
\hline coastal_b5 & Landsat - NASA & $1024 \times 1024$ & 8 \\
\hline coastal_b61 & Landsat - NASA & $512 \times 512$ & 8 \\
\hline coastal_b6h & Landsat - NASA & $512 \times 512$ & 8 \\
\hline coastal_b7 & Landsat - NASA & $1024 \times 1024$ & 8 \\
\hline coastal_b8 & Landsat - NASA & $2048 \times 2048$ & 8 \\
\hline europa3 & Galileo Image from Europa - NASA & $557 \times 600$ & 8 \\
\hline marstest & Mars Pathfinder - NASA & $512 \times 512$ & 8 \\
\hline lunar & Galileo - NASA & $512 \times 512$ & 8 \\
\hline spot-la_b3 & SPOT 3 Imaging - CNES & $500 \times 500$ & 8 \\
\hline spot-la_panchr & SPOT 3 Imaging - CNES & $1000 \times 1000$ & 8 \\
\hline foc & Hubble Space Telescope - NASA & $1024 \times 512$ & 8 \\
\hline ice_2 $2 \mathrm{~kb} 1$ & NOAA Polar Orbiter (AVHRR) - NOAA & $2048 \times 2048$ & 10 \\
\hline ice_ $2 \mathrm{~kb} 4$ & NOAA Polar Orbiter (AVHRR) - NOAA & $2048 \times 2048$ & 10 \\
\hline india_2kb1 & NOAA Polar Orbiter (AVHRR) - NOAA & $2048 \times 2048$ & 10 \\
\hline india_2kb4 & NOAA Polar Orbiter (AVHRR) - NOAA & $2048 \times 2048$ & 10 \\
\hline ocean_2kb1 & NOAA Polar Orbiter (AVHRR) - NOAA & $2048 \times 2048$ & 10 \\
\hline ocean_2kb4 & NOAA Polar Orbiter (AVHRR) - NOAA & $2048 \times 2048$ & 10 \\
\hline landesV_G7_10b & SPOT 5 Imaging - CNES & $454 \times 2381$ & 10 \\
\hline marseille_G6_10b & SPOT 5 Imaging - CNES & $528 \times 1856$ & 10 \\
\hline pleiades_portdebouc_b3 & Simulated PLEIADES - CNES & $1376 \times 320$ & 12 \\
\hline pleiades_portdebouc_pan & Simulated PLEIADES - CNES & $1400 \times 5504$ & 12 \\
\hline solar & Big Bear Solar Observatory - NASA & $1024 \times 1024$ & 12 \\
\hline sun_spot & Big Bear Solar Observatory - NASA & $512 \times 512$ & 12 \\
\hline wfpc & Hubble Space Telescope- NASA & $800 \times 800$ & 12 \\
\hline sar & ERS-1 - ESA & $512 \times 512$ & 16 \\
\hline P_160_B_F & Picard Imager (IAS) - CNRS & $2048 \times 2048$ & 16 \\
\hline
\end{tabular}

Table B.1: CCSDS Reference image set.

\footnotetext{
${ }^{1} \mathrm{xxx}=\mathrm{ESA}, \mathrm{NASA}, \mathrm{CNES}, \mathrm{NOAA}$, or CNRS.
} 

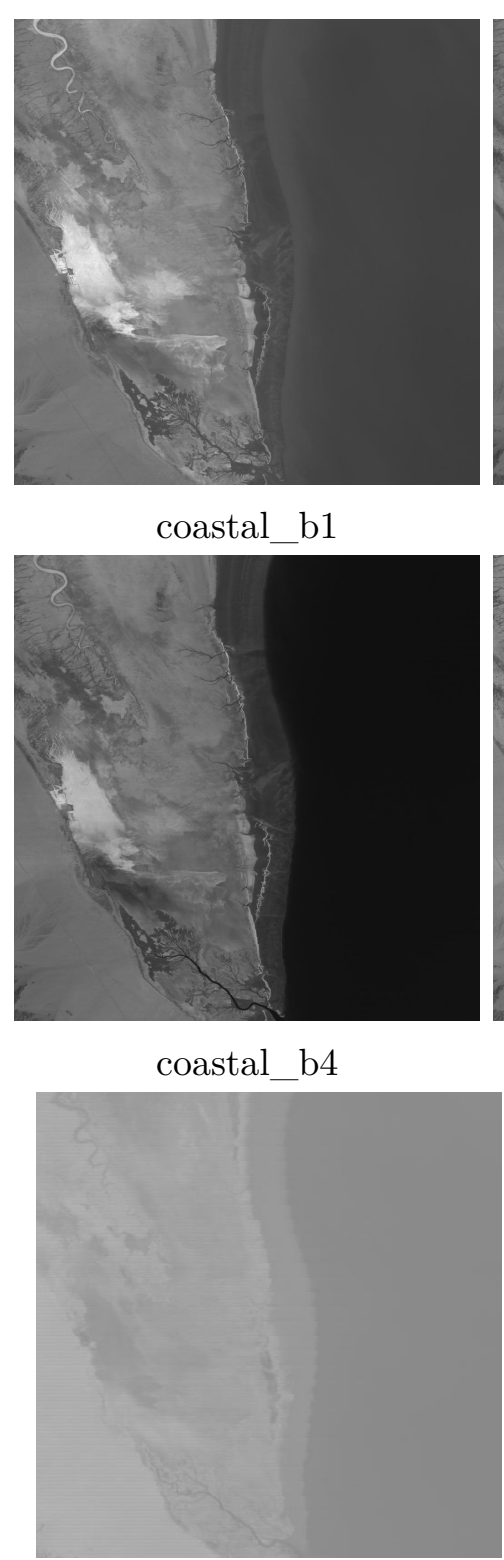

coastal_b61

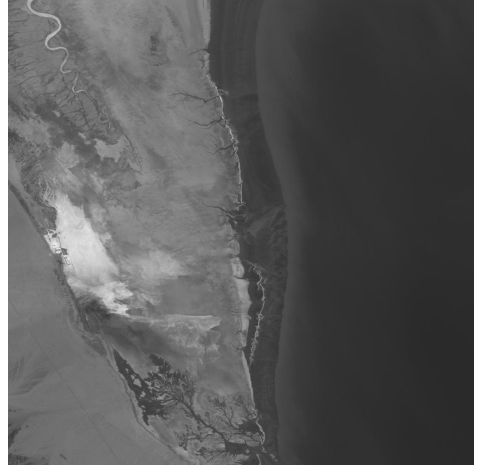

coastal b2

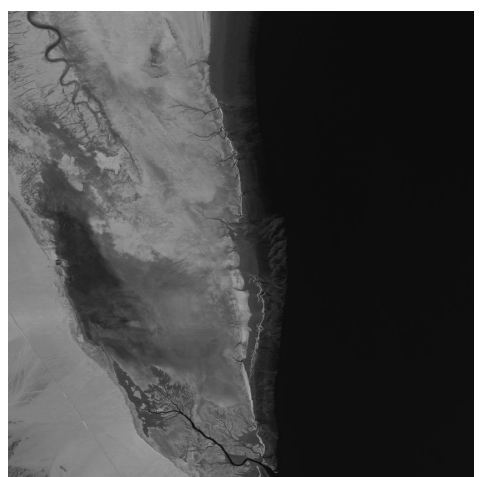

coastal_b5

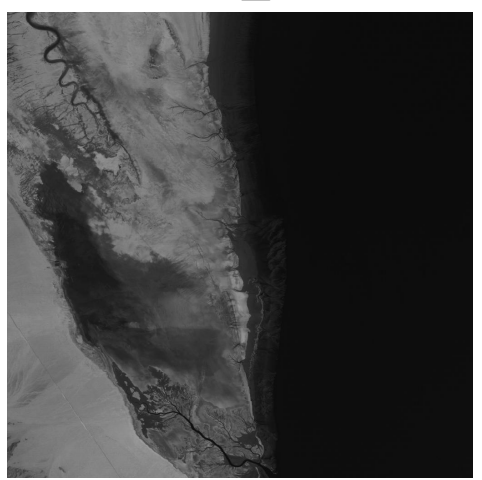

coastal_b7

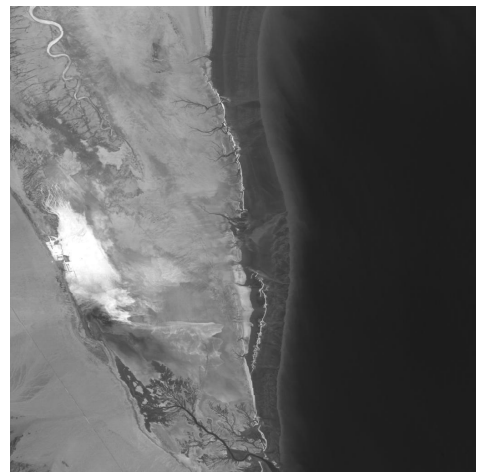

coastal_b3

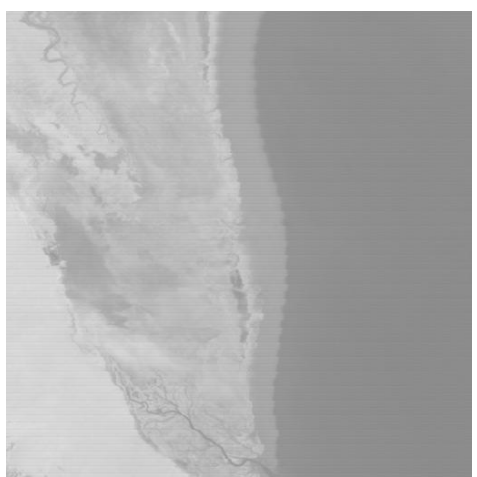

coastal b6h

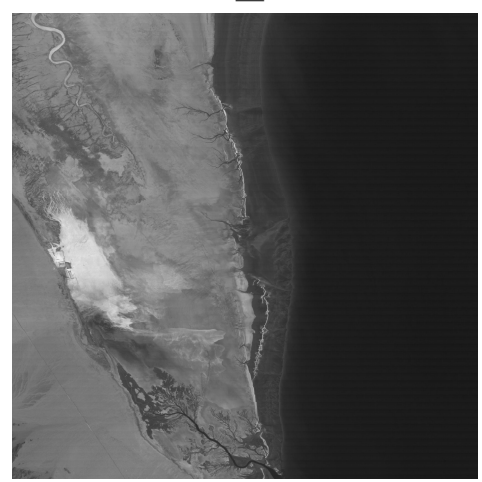

coastal_b8

Figure B.2: coastal, Credit: $C C S D S$. 


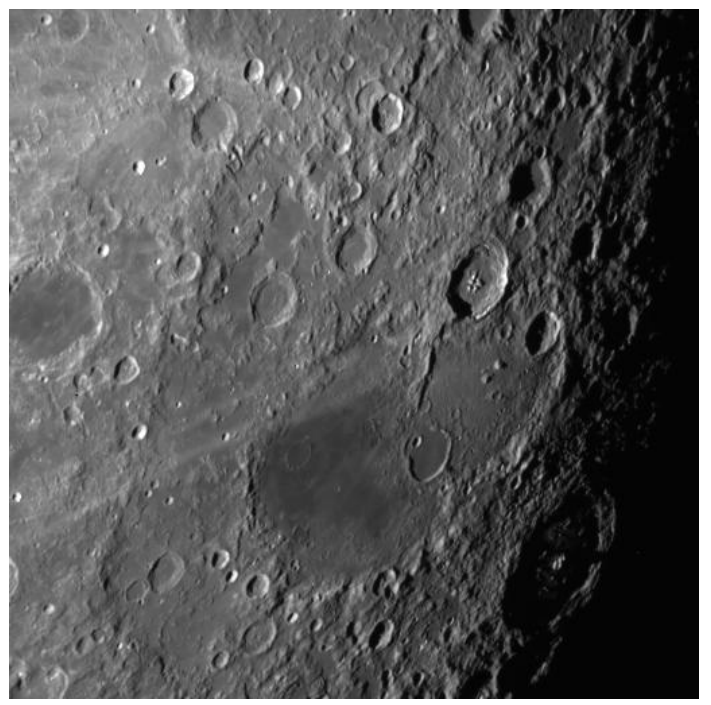

Figure B.3: lunar, Credit: $C C S D S$.

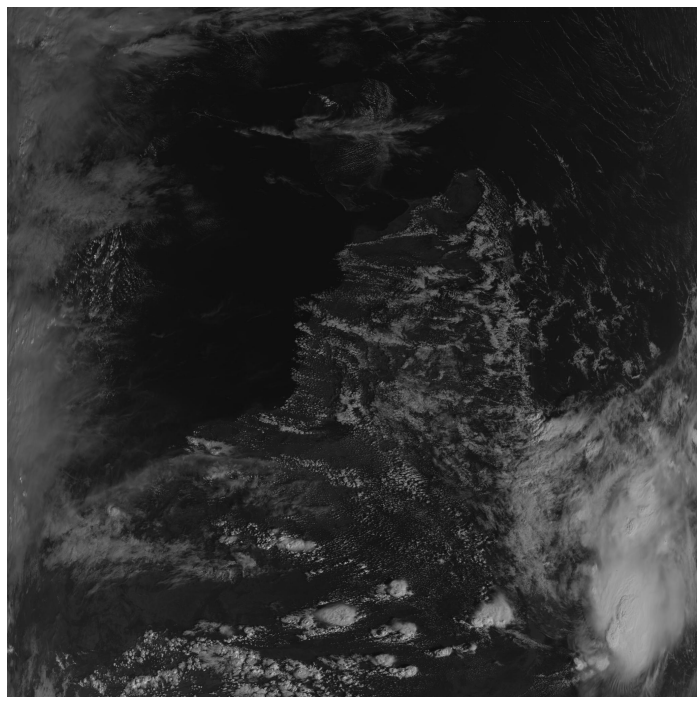

Figure B.5: india_2kb1, Credit: CCSDS.

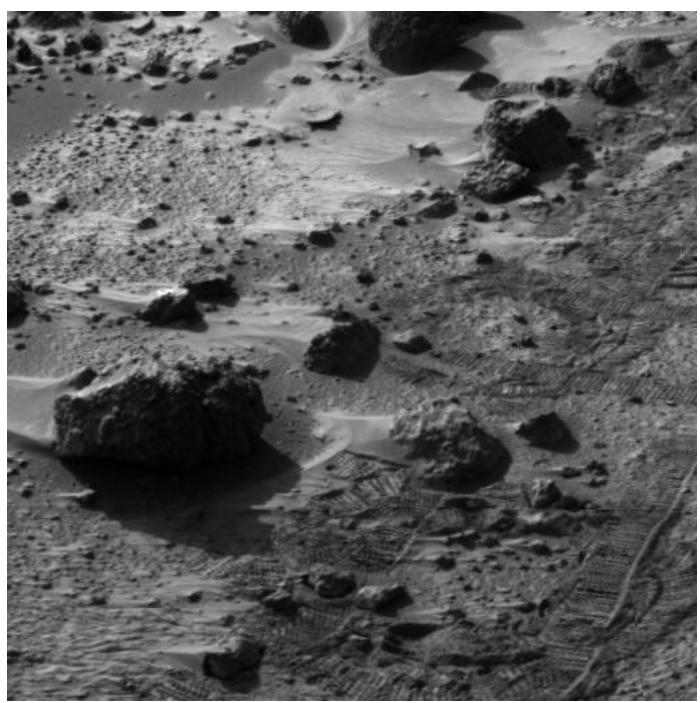

Figure B.4: marstest, Credit: $C C S D S$.

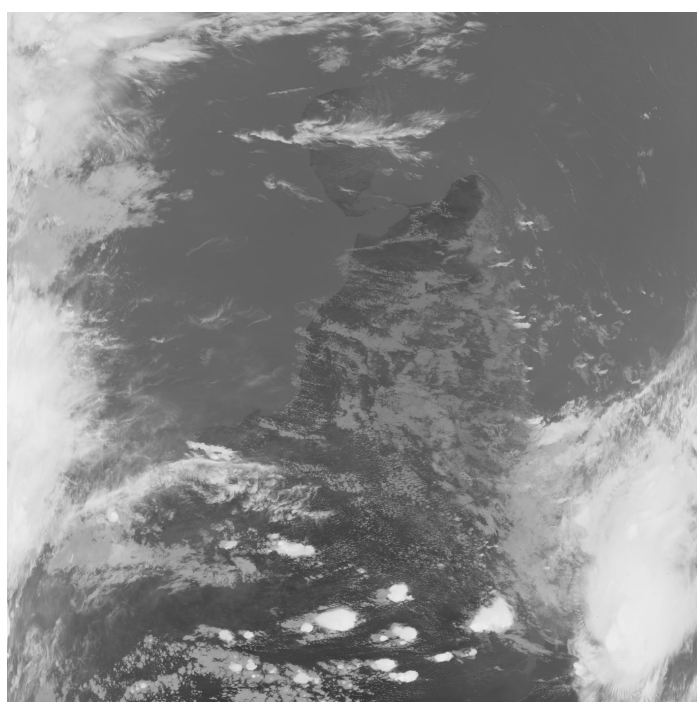

Figure B.6: india_2kb4, Credit: CCSDS. 


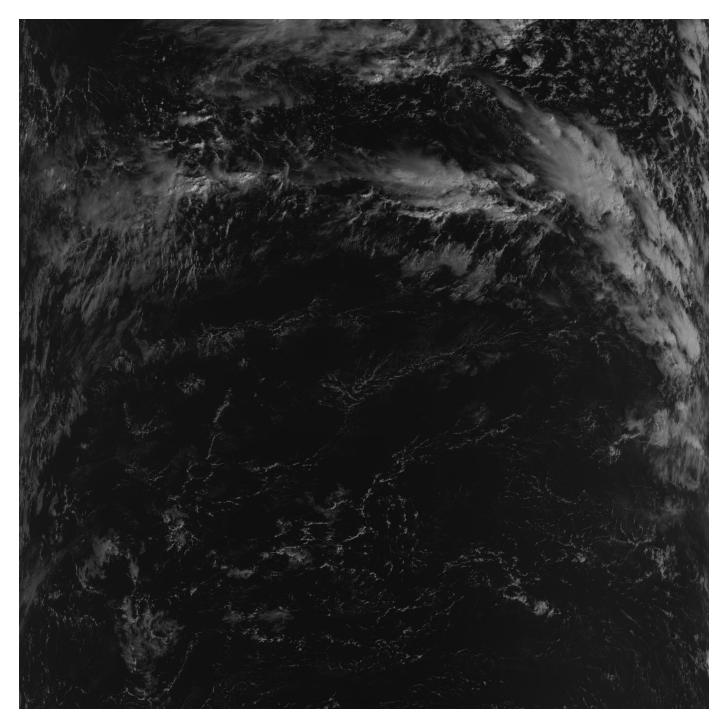

Figure B.7: ocean_2kb1, Credit: $C C S D S$.

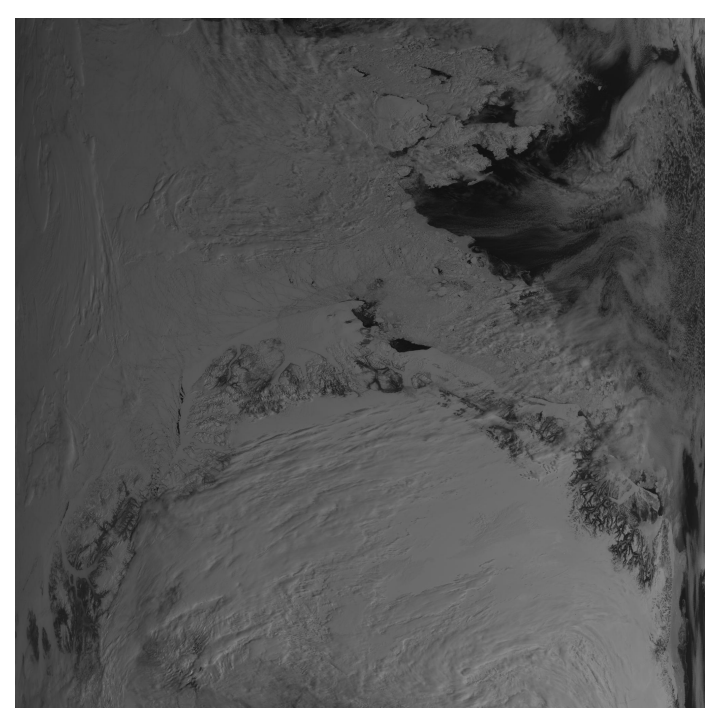

Figure B.9: ice_2kb1, Credit: $C C S D S$.

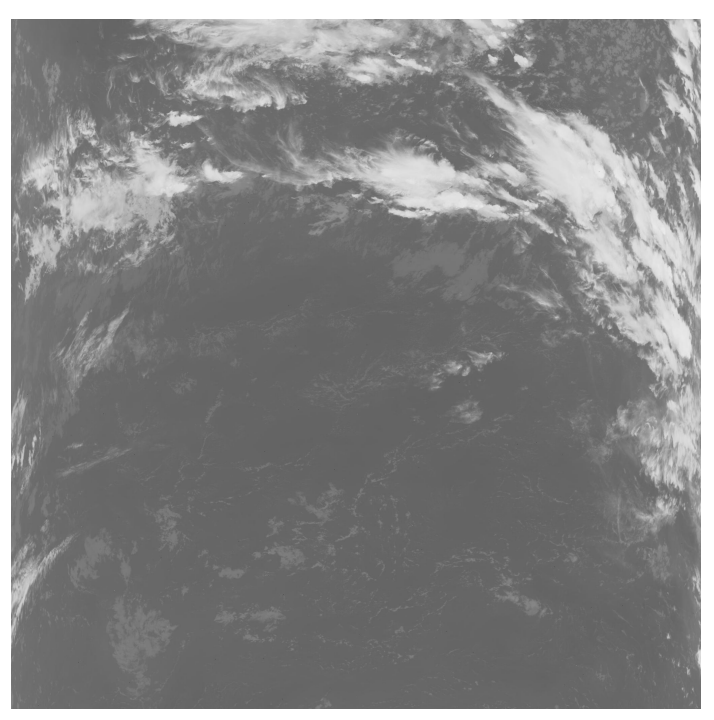

Figure B.8: ocean_2kb4, Credit: $C C S D S$.

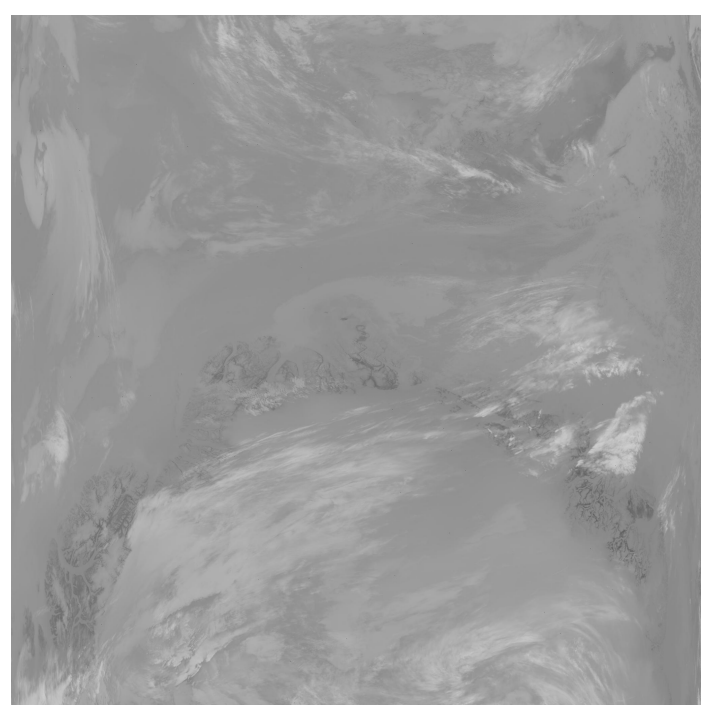

Figure B.10: ice_2kb4, Credit: $C C S D S$. 


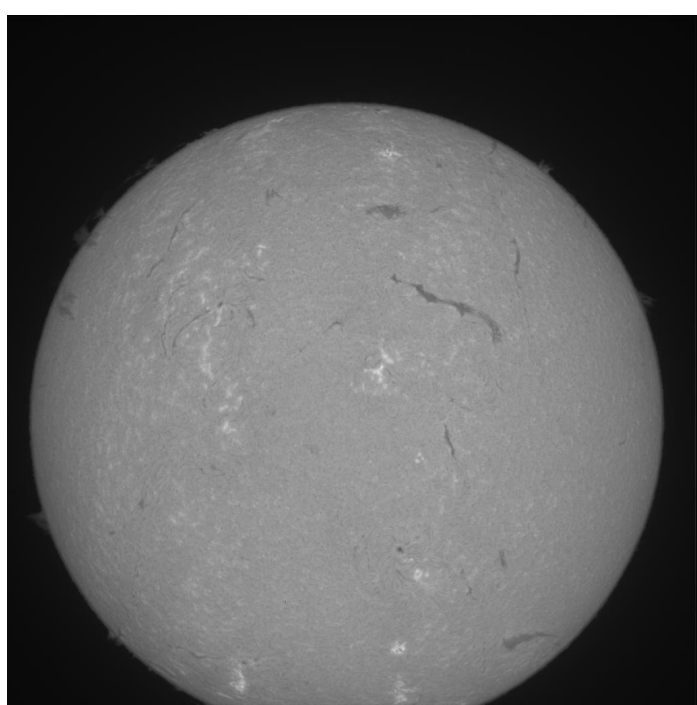

Figure B.11: solar, Credit: $C C S D S$.

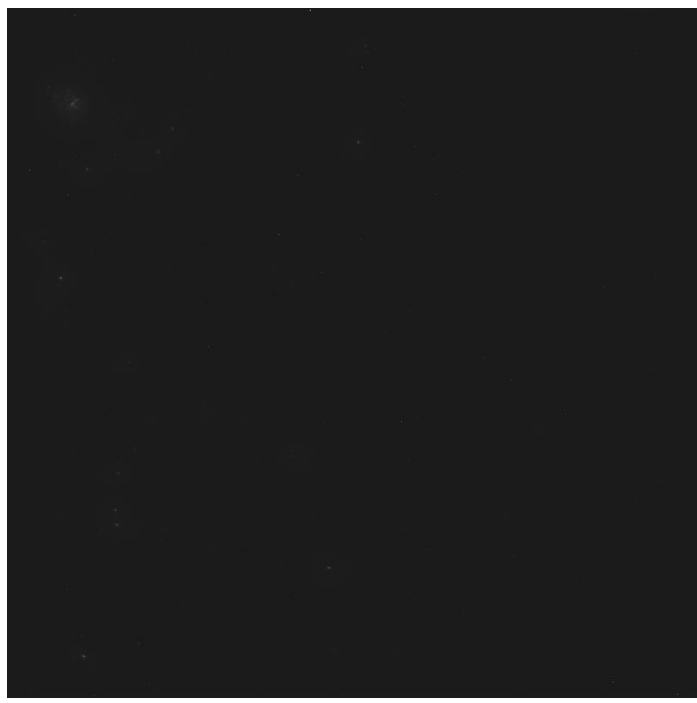

Figure B.13: wfpc, Credit: $C C S D S$.

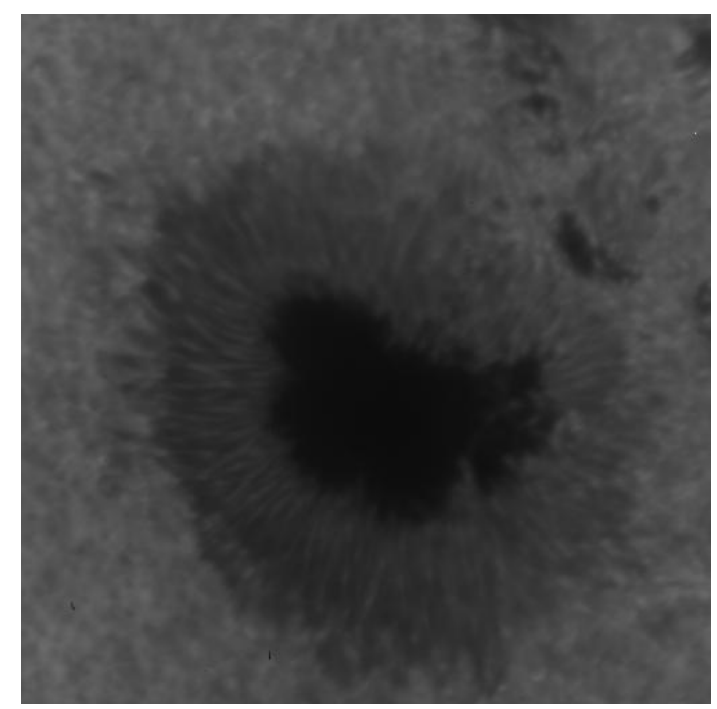

Figure B.12: sun_spot, Credit: $C C S D S$.

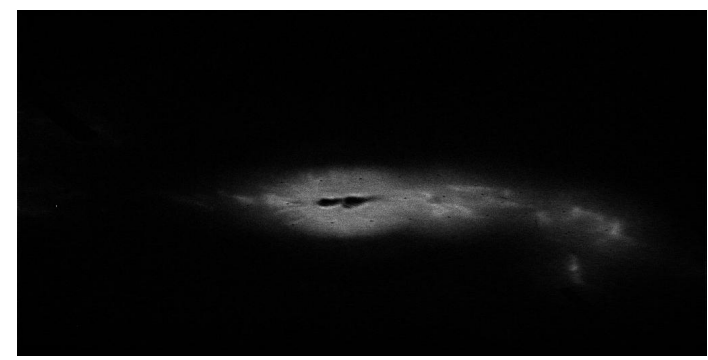

Figure B.14: foc, Credit: $C C S D S$.

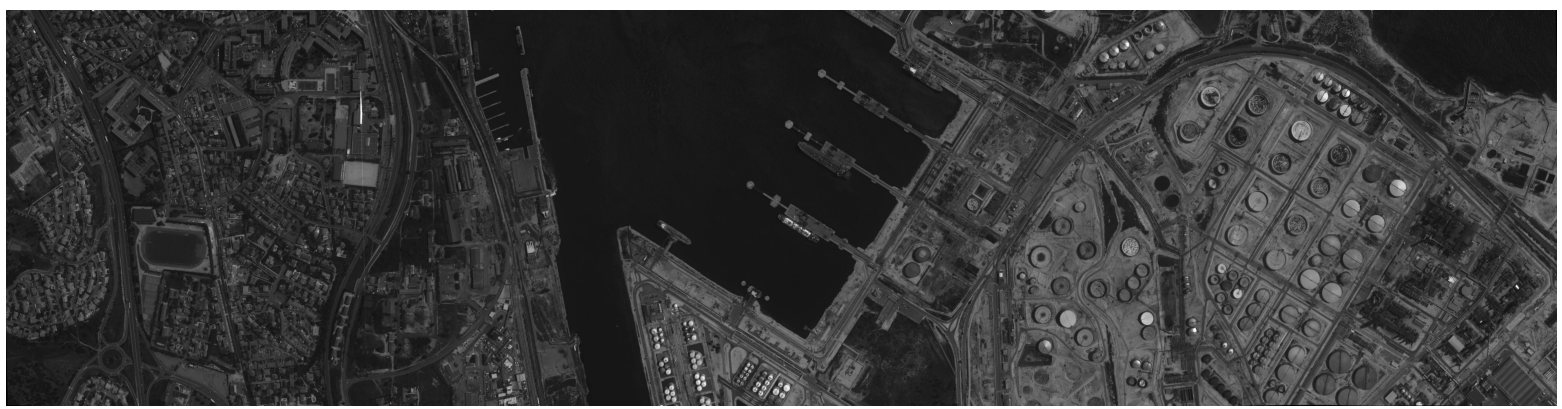

Figure B.15: pleiades_portdebouc_pan, Credit: $C C S D S$. 


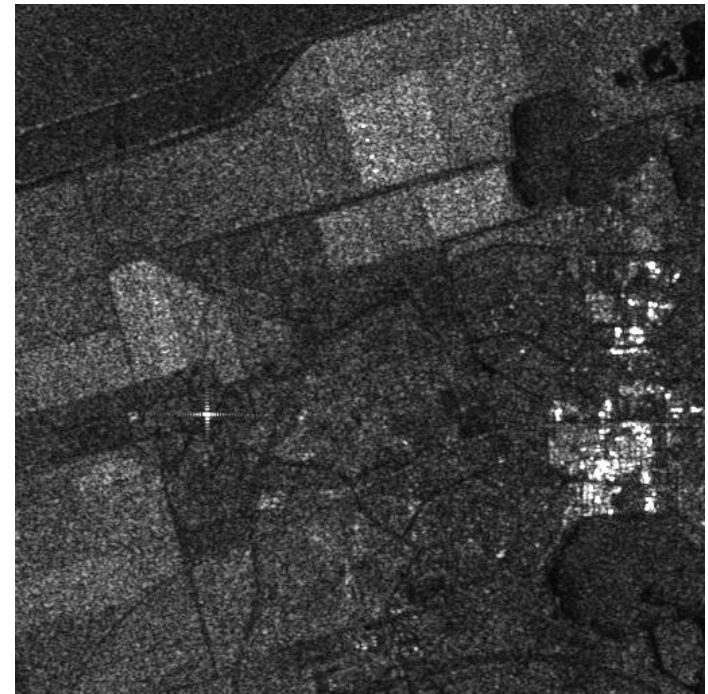

Figure B.16: sar, Credit: $C C S D S$.

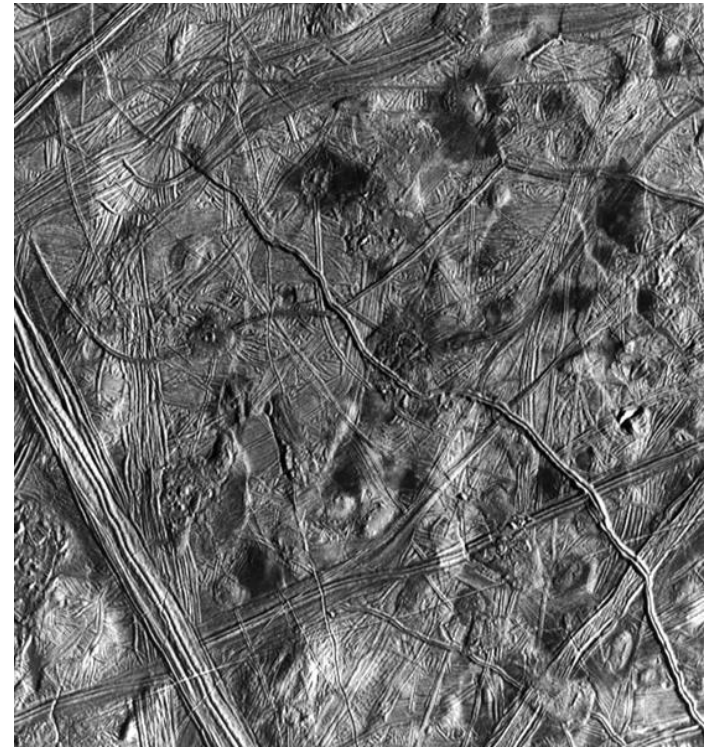

Figure B.18: europa3, Credit: $C C S D S$.

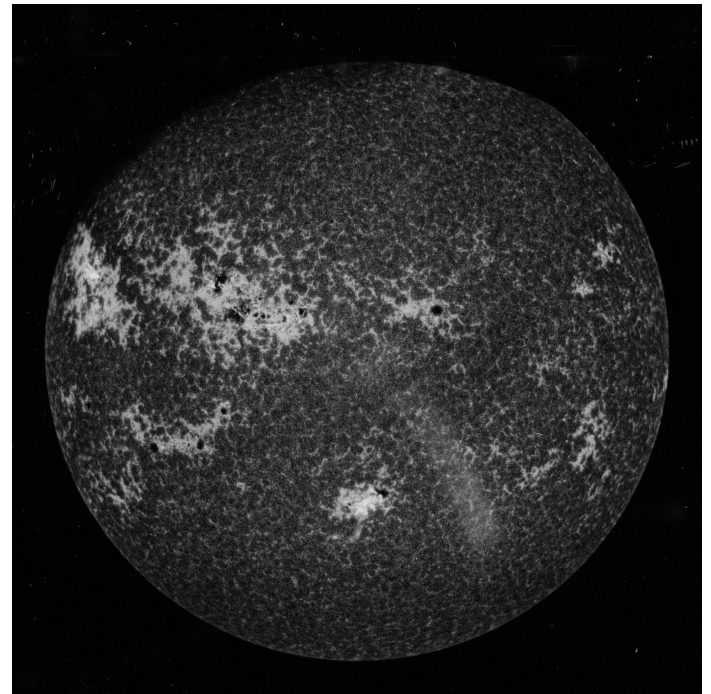

Figure B.17: p160_b_f, Credit: $C C S D S$.

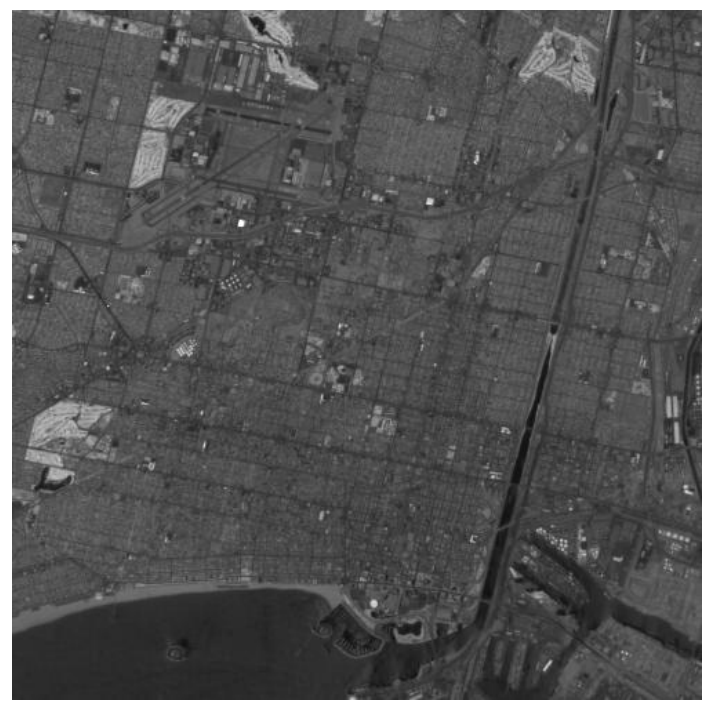

Figure B.19: spot-la_b3, Credit: $C C S D S$.

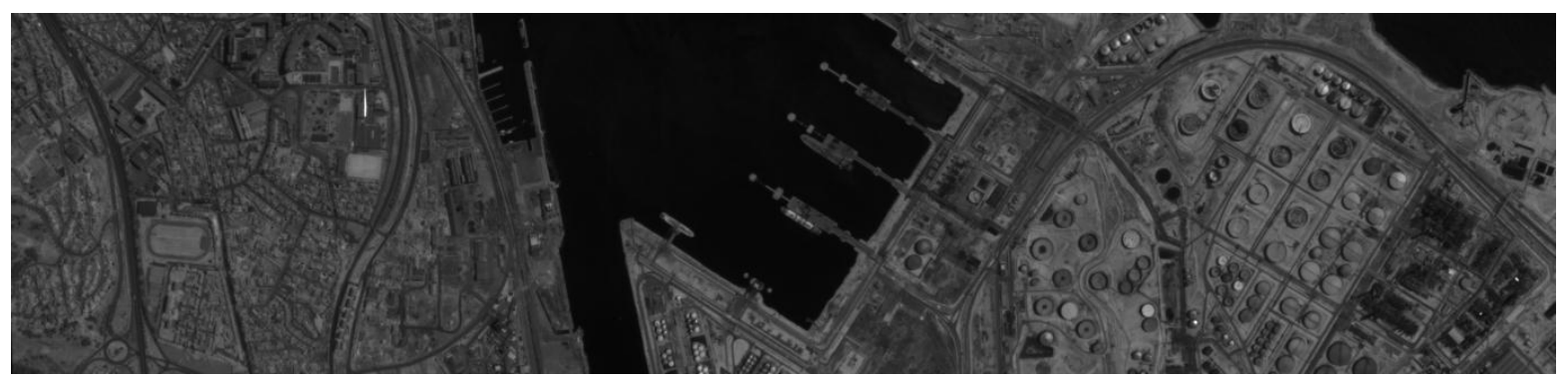

Figure B.20: pleiades_portdebouc_b3, $C C S D S$ 


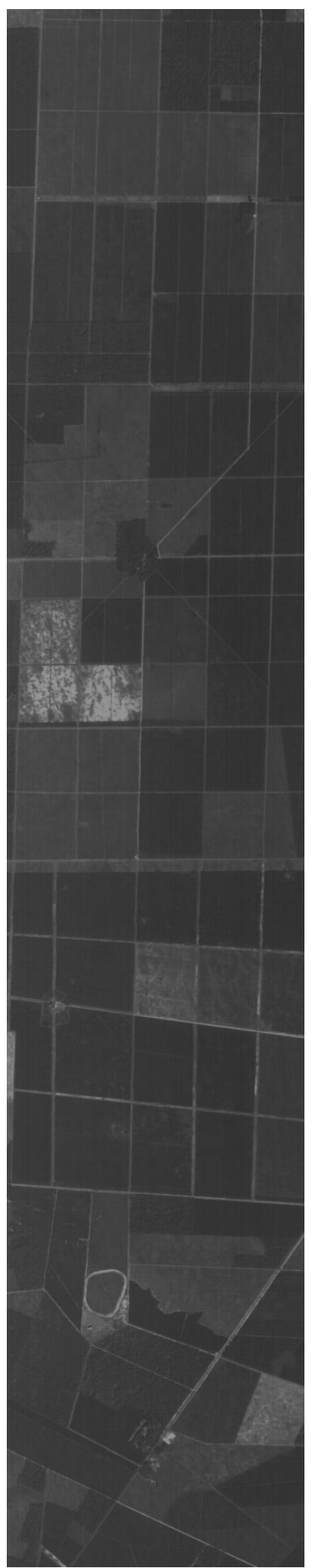

Figure B.21: landesV_G7_10b, Credit: $C C S D S$.

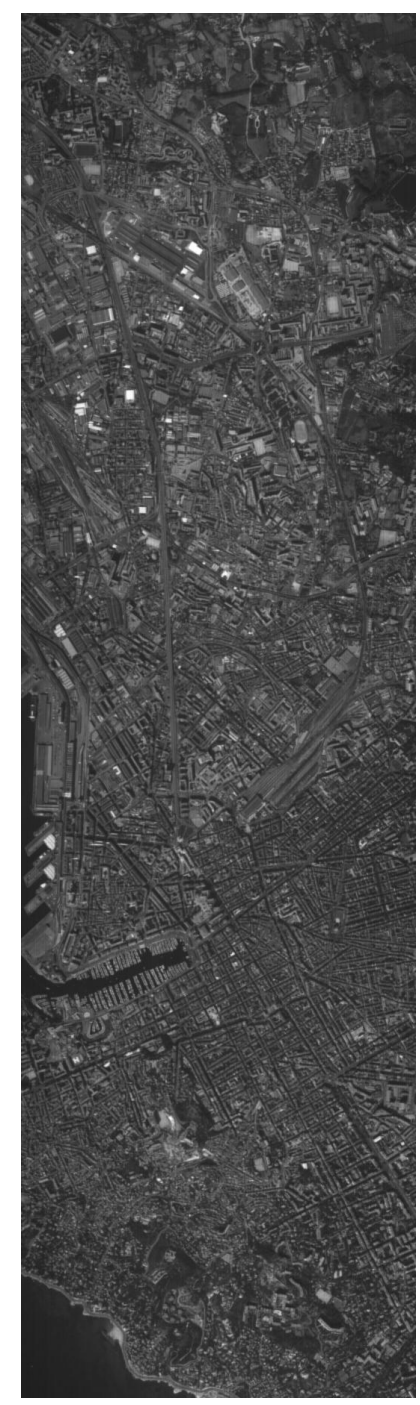

Figure B.22: marseille_G6_10b, Credit: $C C S D S$. 


\section{B.5 AVIRIS Hyper-Spectral Images}

The most widely used images for benchmarking hyper-spectral image compression algorithms are the standard 1997 AVIRIS calibrated radiance images [56, 57].

Calibrated and uncalibrated AVIRIS images have been provided to the Consultative Committee for Space Data Systems (CCSDS) Multi-spectral and Hyperspectral Data Compression (MHDC) working group for compression testing and evaluation. This newer AVIRIS data, summarized in the table below, consists of five calibrated and corresponding 16-bit raw images acquired over Yellowstone, WY in 2006, as well as two additional 12 bit uncalibrated images. Each image is a 512 line scene containing 224 spectral bands.

\begin{tabular}{lllllll}
\hline Site & Scene Numbers & Flight & Year & Size $(\mathbf{h} \times \mathbf{w} \times \mathbf{d})$ & Dynamic range [bpp] & Data Type \\
\hline Yellowstone, WY & $0,3,10,11,18$ & f060925t01p00r12 & 2006 & $512 \times 680 \times 224$ & 16 & uncalibrated \\
\hline Yellowstone, WY & $0,3,10,11,18$ & f060925t01p00r12 & 2006 & $512 \times 677 \times 224$ & 16 & calibrated \\
\hline Hawaii & 1 & f011020t01p03r05 & 2001 & $512 \times 614 \times 224$ & 12 & uncalibrated \\
\hline Maine & 10 & f030828t01p00r05 & 2003 & $512 \times 680 \times 224$ & 12 & uncalibrated \\
\hline
\end{tabular}

Table B.2: AVIRIS hyper-spectral images. 



\section{Acknowledgment}

This thesis is about my work as a scholarship holder at the DLR. I would like to thank Helmholtz Research School on Security Technologies (HRSST) for their financial support and the opportunity to work at the DLR. The HRSST is a joint program of Technische Universität Berlin (TUB) and DLR. It is a PhD program about the investigation of the civil security technologies in Germany, which had started in August 2010.

I would also like thank

- all my colleagues at DLR, especially Prof. Dr. Ralf Reulke, Dr. Anko Börner, Dr. Andreas Eckardt, Dr. David Krutz, Dr. Harald Michaelis, Ilse Sebastian, and Dominik Rueß for many constructive discussions,

- Prof. Dr. Ben Juurlink, who is my supervisor at TUB, PhD. Ahmed Elhossini, and Jan Lucas,

- Prof. Dr. Meffert and Dr. Frank Winkler from the Humboldt-Universität zu Berlin (HUB),

- Sunil Chokkanathapuram Ramanarayanan, who worked on a GPGPU implementation of the CCSDS 122.0-B-1 standard,

- all who have read this thesis and gave me feedback,

- and my family. 



\section{Declaration}

I declare that this thesis has been composed by myself, that the work contained herein is my own except where explicitly stated otherwise, and that this work has not been submitted for any other degree or professional qualification except as specified.

Hohen Neuendorf, March 10, 2017 